\title{
Mind the gatekeeper
}

Citation for published version (APA):

Goncalves Magro Dos Reis, I. A. (2022). Mind the gatekeeper: key role of NPC1 protein and lysosomal cholesterol in metabolic diseases and therapeutical implications. [Doctoral Thesis, Maastricht University]. Gildeprint Drukkerijen. https://doi.org/10.26481/dis.20220126im

Document status and date:

Published: 01/01/2022

DOI:

10.26481/dis.20220126im

Document Version:

Publisher's PDF, also known as Version of record

\section{Please check the document version of this publication:}

- A submitted manuscript is the version of the article upon submission and before peer-review. There can be important differences between the submitted version and the official published version of record.

People interested in the research are advised to contact the author for the final version of the publication, or visit the DOI to the publisher's website.

- The final author version and the galley proof are versions of the publication after peer review.

- The final published version features the final layout of the paper including the volume, issue and page numbers.

Link to publication

\footnotetext{
General rights rights.

- You may freely distribute the URL identifying the publication in the public portal. please follow below link for the End User Agreement:

www.umlib.nl/taverne-license

Take down policy

If you believe that this document breaches copyright please contact us at:

repository@maastrichtuniversity.nl

providing details and we will investigate your claim.
}

Copyright and moral rights for the publications made accessible in the public portal are retained by the authors and/or other copyright owners and it is a condition of accessing publications that users recognise and abide by the legal requirements associated with these

- Users may download and print one copy of any publication from the public portal for the purpose of private study or research.

- You may not further distribute the material or use it for any profit-making activity or commercial gain

If the publication is distributed under the terms of Article $25 \mathrm{fa}$ of the Dutch Copyright Act, indicated by the "Taverne" license above, 


\section{Mind the gatekeeper:}

Key role of NPC1 protein and lysosomal cholesterol in metabolic diseases and therapeutical implications

Inês Andreia Gonçalves Magro dos Reis 
(C) Inês Reis, Maastricht 2022

All rights reserved. No part of this thesis may be reproduced or transmitted in any form or by any means, electronic or mechanical, including photocopying, recording or any information storage of retrieval system, without written permission from the author.

ISBN:

Cover design: Inês Reis

Cover illustration: renikca/Shutterstock.com, used under standard license Thesis layout: Inês Reis

Printed by: Gildeprint - Enschede, www.gildeprint.nl

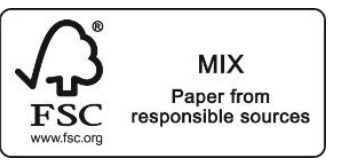

The studies presented in this thesis were performed within the school of NUTRIM (Nutrition and Translational Research in Metabolism) at Maastricht University, The Netherlands.

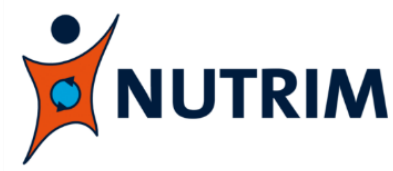

The research presented in this thesis was supported by a TKI grant. 


\section{Mind the gatekeeper:}

Key role of NPC1 protein and lysosomal cholesterol in metabolic diseases and therapeutical implications

\section{DISSERTATION}

to obtain the degree of Doctor at Maastricht University,

on the authority of the Rector Magnificus, Prof.dr. Rianne M. Letschert, in accordance with the decision of the Board of Deans,

to be defended in public on Wednesday, $26^{\text {th }}$ of January 2022, at 13:00 hours

by

Inês Andreia Gonçalves Magro dos Reis

Born on the $28^{\text {th }}$ of February 1993, in Lisbon, Portugal 


\section{Promotor:}

Prof. Dr. Ronit Shiri-Sverdlov

\section{Co-promotor:}

Dr. Tom Houben

\section{Assessment committee:}

Prof. Dr. Frederik-Jan van Schooten (chair)

Dr. Sabine Baumgartner

Prof. Dr. David Leake (University of Reading, United Kingdom)

Dr. Kaatje Lenaerts

Dr. Frank Pfrieger (University of Strasbourg, France)

Prof. Dr. Marc van Zandvoort 


\section{TABLE OF CONTENTS}

Chapter 1 General introduction

page 7

Chapter 2 Effects of dietary phytostanol supplementation on

peripheral symptoms in a mouse model for Niemann-Pick page 29 type $\mathrm{C} 1$ disease.

Chapter 3 Anti-inflammatory effects of dietary plant stanol supplementation are largely dependent on the intake of page 53 cholesterol in a mouse model of metabolic inflammation.

Chapter 4 Hematopoietic Npc1 mutation shifts gut microbiota composition in $\mathrm{Ldlr}^{--}$mice on a high-fat, high-cholesterol page 73 diet.

Chapter 5 Pneumococcal Immunization Reduces Neurological and Hepatic Symptoms in a Mouse Model for Niemann-Pick page 101 Type C1 Disease.

Chapter 6 The Influence of a Conjugated Pneumococcal Vaccination on Plasma Antibody Levels against Oxidized Low-Density Lipoprotein in Metabolic Disease Patients: A Single-Arm page 133 Pilot Clinical Trial.

Chapter 7 General discussion page 149

Addendum Summary page 166

Samenvatting page 168

Impact paragraph page 170

Acknowledgements page 175

Curriculum vitae Page 180 

Chapter 1

General introduction 


\section{Overture: the importance of lipids and lipid metabolism dysfunction}

From whales to bacteria, all beings depend on the tight balance between the synthesis, breakdown and processing of biomolecules to support their growth and replication, including nucleic acids, proteins, carbohydrates and lipids. Lipids consist of a heterogeneous group of water-insoluble and organic-soluble molecules, which perform a wide range of biological functions. At the cellular level, lipids are essential for membrane structure and function, and are also important substrates for energy production and signaling molecules. In multicellular organisms, lipids also contribute to thermal regulation, energy storage and interorgan communication. Given that lipids play extensive and important roles in physiological processes, it is not surprising that lipid metabolism is a complex and tightly regulated process, both at whole-body and at the cellular level. Indeed, dysfunctions of lipid metabolism, even if subtle, trigger a domino-like cascade of events that culminate in the development of severe metabolic disorders.

For years, it has been inevitable to find oneself gazing at expressions such as "global obesity/metabolic syndrome epidemic" time and time again. Far from a cliché, such words accurately describe the reality of more and more individuals, whose lifestyle often propels the development of severe and fatal disorders (1). In parallel with reduced rates of physical activity, easy access to highly caloric and nutrient-poor foods has translated into the seemingly ever-increasing prevalence of overweight and obesity worldwide among children and adults alike. In addition to unhealthy lifestyles, patients suffering from a wide range of genetic diseases are predisposed to overweight and metabolic dysfunction. Regardless of the underlying etiology, metabolic disorders often feature lipid metabolism dysfunction as a pathophysiological mechanism. Importantly, overweight and metabolic disorders are major risk factors for the development of non-communicable diseases such as cardiovascular and liver disease, dementia and cancer, which account for nearly $70 \%$ of preventable deaths worldwide (2). Although the dangers of infectious diseases were once again in the spotlight in 2020, there is no doubt that metabolic disorders and associated disease burden consist of some of the largest threats to public health and will remain so in years to come. In order to develop new therapeutic strategies and counterbalance morbidity rates associated with metabolic disorders, there is a pressing need to explore key players in lipid metabolism, in health and disease.

In this General Introduction, I will provide an overview of whole-body lipid metabolism. Subsequently, I will focus on the roles mediated by lysosomes and the lysosomal NiemannPick type C1 (NPC1) protein in lipid metabolism. Finally, I will emphasize the consequences of lysosomal and NPC1 impairment and their involvement in metabolic disorders. 


\section{Lipid metabolism and trafficking: to the liver and beyond}

Throughout their lives, individuals will consume a wide variety of dietary sources of lipids, ranging from grilled fish to avocados, pizza and dark chocolate. Regardless of the meal, dietary lipids must be broken down and ultimately absorbed via the gastrointestinal tract, a process made complex due to the intrinsic hydrophobia of lipids and the watery nature of most body fluids. As our food reaches the duodenum and the small intestine, enzymes and bile acids collaborate to stabilize lipid emulsions and further break down lipid molecules into free fatty acids (FFAs), monoglycerides, lysophospholipids and free cholesterol. Dietary and bile acid-derived lipids are ultimately internalized by the intestinal enterocytes, where they are further processed and shipped as chylomicrons to the lymphatic system in order to finally reach the liver, the body's metabolic hub $(3,4)$.

A proverbial "Jack of all trades", the liver contributes to a plethora of physiological needs, including storage and synthesis of glucose, lipids and proteins/amino acids; regulation of glucose and lipid blood levels; maintenance of optimal blood volume; production of bile acids and maintenance of intestinal absorption of lipids, fat-soluble vitamins and minerals; regulation of immune responses; and the processing and excretion of drugs and xenobiotic and biological waste molecules $(5,6)$. A variety of cells cooperate to support liver functions, namely, hepatocytes, the most abundant liver cell type and considered its main effector cell; Kupffer cells, the liver's resident macrophages; stellate cells, which contribute to vitamin storage and scar tissue formation in case of injury; and biliary epithelial cells and endothelial cells, which specialize in bile production and selective molecule exchange, respectively (7). In addition to its cellular composition, the liver's central position, lobular organization and unique dual blood supply make it well tailored to perform the array of functions mentioned above.

After every meal, intestine-derived nutrients and chylomicrons reach the liver via the hepatic vein, where the lipid contents of chylomicrons are internalized by the hepatocytes. Here, FFAs, phospholipids and cholesterol esters can either be stored for later use in cytosolic lipid droplets or metabolized according to the needs of hepatocytes. In addition, dietary lipids are also packaged by hepatocytes in very low-density lipoproteins (VLDLs), triglyceride (TG)enriched particles stabilized by a coating of apolipoproteins, cholesterol and phospholipids, which are then shipped into the circulation $(8,9)$. As VLDLs make their way through the body, they eventually make contact with VLDL receptors, produced mainly by energydemanding tissues such as the heart and muscle, as well as by the adipose tissue, a prime extra-hepatic site for fat storage. Once anchored in place by VLDL receptors, VLDL-derived TGs are released and hydrolyzed into FFAs by lipoprotein lipases and internalized by the cells on site. As VLDLs progressively unload their lipid cargo throughout the body, they 
become smaller and poorer in TGs, thus transitioning into IDLs. In turn, IDL particles are further enriched in cholesterol captured from HDL and depleted of TGs, originating LDL, the body's main cholesterol carrier. The interaction of LDL's apolipoproteins with cells via LDL receptors results in the release of LDL contents into the cells for further processing or storage. Conversely, excess cellular lipids are secreted by the various organs and tissues into circulating HDL and delivered back to the liver, where the contents can be further processed for usage (fi, bile acid production), storage or excretion in feces $(3,10)$.

Notably, unlike other organs and tissues, the nervous system is not dependent on liverderived V- and LDLs to receive its share of lipids, as these particles hardly cross the bloodbrain barrier (BBB) (11). Although the brain usually uses glucose to meet its high energy requirements, it is one of the fattiest organs in the body and it needs high levels of lipids, particularly cholesterol, to maintain neurotransmission and axon myelination, as well as to support constant synthesis and remodeling of synapses. While, as aforementioned, the brain is mostly impermeable to lipoproteins, it receives esterified and free FAs carried by liverproduced albumin in the blood. Once FAs cross the BBB, they are carried to astrocytes, arguably the brain's most abundant cell type. In addition to receiving and processing FAs from the blood circulation, astrocytes oversee cholesterol de novo synthesis in the brain and lipoprotein-mediated lipid delivery to neurons and other cells within the nervous system (12). While lipid deficiencies can severely compromise proper brain function, excessive lipid levels may also lead to cellular dysfunction and death. In order to avoid lipotoxicity, brain cells, particularly neurons, convert surplus cholesterol to 24S-hydroxycholesterol, which transverses the BBB much easier than cholesterol (13). 24S-hydroxycholesterol then exits the nervous system following incorporation into peripheral HDL and, to a lesser extent, LDL particles, making its way into the liver, where it is finally excreted as bile. For an overview of lipid trafficking across the body, please refer to Figure 1.

Although whole body lipid metabolism is an extensive process on its own, the fact that it must be enacted in coordination with glucose and amino acid metabolism adds another layer of complexity to the process. Although it is beyond the scope of this Introduction (and $\mathrm{PhD}$ thesis in general) to paint a complete picture of whole-body metabolism, it is important to emphasize the role of organ crosstalk in whole-body metabolism, as deficiencies in one process or another can - and do - trigger a "house of cards" effect in metabolic disorders.

While, as aforementioned, the liver is essential in regulating whole-body metabolism at all levels, it is not alone in this herculean task. In times of abundance, for instance after a meal, increased blood glucose levels prompt the release of insulin by the pancreas; conversely, low glucose levels during fasting or in between meals trigger the release of glucagon, a hormone 
that counteracts the effects of insulin. Overall, activation of insulin receptors in the liver and other organs stimulates the uptake, storage and use of glucose as fuel, suppressing its synthesis. In parallel, insulin activity inhibits lipolysis in the adipose tissue, thus stimulating the synthesis and storage of TGs in lipid droplets for use when glucose levels plummet and glucagon activity increases. Dysregulation of glucose and/or FA levels and metabolism in obesity and metabolic disorders is often associated with impaired response to insulin, resulting in high blood glucose levels (14). In addition, abnormal insulin activity leads to increased TG synthesis, which further contributes to excess cellular lipid accumulation and secretion, increasing VLDL and LDL levels in the blood (15). In addition to pancreatic hormones, the brain, adipose tissue and muscle also sense nutrient levels and in turn regulate whole-body metabolism by a variety of mechanisms, most notably via the release of a variety of hormones that ultimately enhance or suppress appetite and regulate lipid and glucose catabolism and anabolism (16-18).

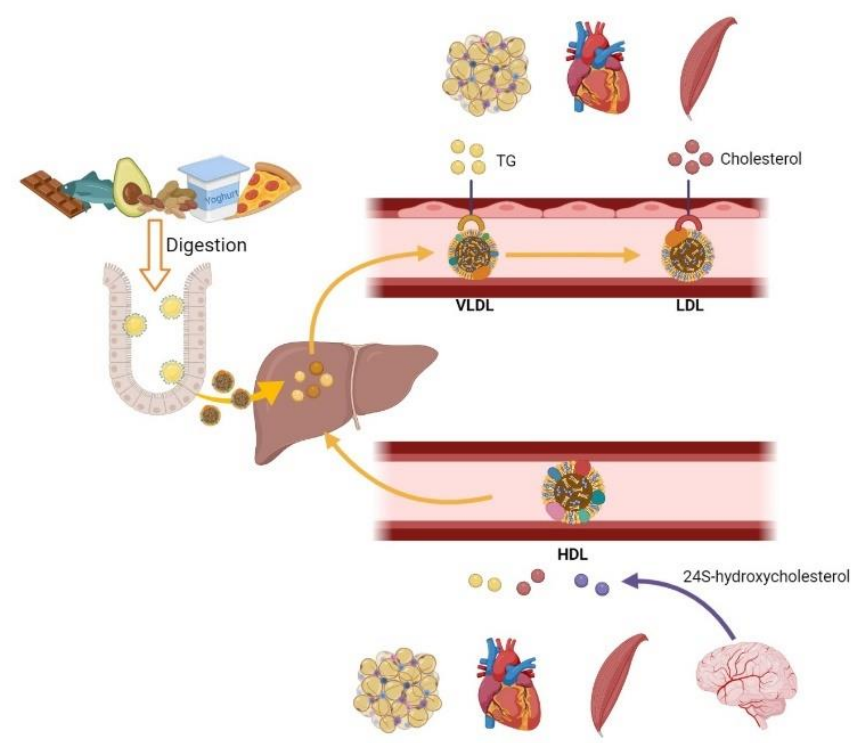

Figure 1 Overview of whole-body lipid trafficking. Dietary lipids are broken down in the gastrointestinal tract to be absorbed in the small intestines and shipped into the liver as chylomicrons. Hepatic cells metabolize TGs, phospholipids and cholesterol and package them into VLDL particles, which travel along the circulation in order to reach other organs, such as the heart, muscles and adipose tissue. As VLDLs shed their TG content along the way and become enriched in cholesterol, they become LDL, the body's main cholesterol carrier. Unlike the majority of organs and tissues, the nervous system is mostly impermeable to circulating V-and LDL; instead, brain cells meet their lipid needs by sequestering albumin-bound circulating FAs and producing cholesterol de novo. Excess cholesterol, converted to 24Shydroxycholesterol in the case of the brain, is excreted and incorporated into circulating HDL and returned to the liver, where it can be further processed for storage or excretion in the large intestines. This figure was created with BioRender.com. 
Finally, in addition to organs and self-derived cells and molecules, our gut microbiome is increasingly implicated in the regulation of lipid metabolism. While largely ignored for decades, a growing body of research, aided by the advent of gene sequencing, has identified thousands of species of micro-organisms in the gut. Similarly to a fingerprint, the microbiome composition is a highly personal affair, dependent on the individual's genes, age, metabolism and history of infections, as well as on the diet and exposure to environmental factors and drugs (14). Despite interpersonal variations, studies have shown that different structures and organs (fi, skin, mouth, vagina, gut) within large human populations display characteristic microbe compositions. Importantly, shifts in microbiome composition are associated with diseases such as Parkinson's disease, depression, liver disease and cancer (15). Although many efforts are ongoing to establish a causal relationship between the microbiome composition and disease development, researchers have determined that a healthy gut microbiome contributes to our welfare, for instance, by preventing the growth and spread of foreign pathogens; aiding in the breakdown and absorption of nutrients; and regulating immune responses and metabolism (14).

Overall, optimal whole-body lipid metabolism aims to balance the amount of lipids produced, stored and used by the cells for structural and energy-producing purposes after and in between meals; as well as to keep the amount of lipids in the circulation within a strict physiological range, thus avoiding lipid deficiencies or lipotoxicity. Proper function and collaboration among a wide array of cell types, including hepatocytes, pancreatic $\beta$ cells and adipocytes is essential and underlies the network of whole-body lipid metabolism. At the cellular level, lysosomes are also emerging as key players in lipid metabolism, with effects that can impact whole-body processes.

\section{Lysosomes and lysosomal NPC1 as regulators of cellular cholesterol trafficking and metabolism}

Throughout their lifetime, cells in our bodies will continuously send and receive nutrients and signaling molecules, thus mutually regulating their activity for the common good. Depending on the size, electric charge and hydrophilia of the cargo, molecules can cross cellular membranes by passive or facilitated diffusion (in case of small hydrophobic molecules moving along their concentration gradient); active transport (in which large and/or hydrophilic molecules are bound to protein transporters and carried along or against their concentration gradient); and by endo- and exocytosis, in which incoming and outgoing molecules, respectively, make their way in or out of cells within membrane-bound vesicles. In the case of LDL uptake by cells, for instance, LDL must first interact and attach to LDL receptors anchored at the extracellular side of the cell's membrane. Subsequently, the plasma membrane at the site of LDL/LDL-receptor complex folds onto itself and "pinches off", thus 
forming a membrane-bound vesicle (early endosome). Proton pumps incorporated into the membrane of early endosomes lower the vesicle's $\mathrm{pH}$ to 6.0, causing the dissociation of LDL and its receptor (16). Subsequently, the LDL receptor is incorporated into a different vesicle and recycled back into the membrane, whereas the LDL-containing vesicle will fuse together with other endosomes and, ultimately, with a lysosome (Figure 2).

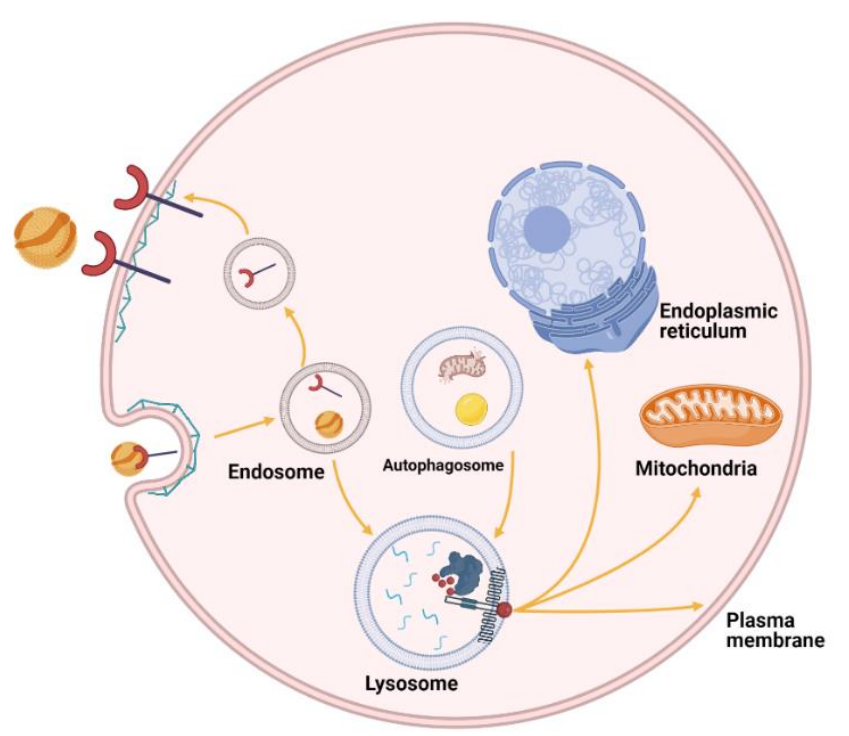

Figure 2 Overview of NPC1-mediated cholesterol processing in lysosomes. Circulating LDL makes contact with a cellular LDL receptor, forming a LDL-LDL receptor complex that undergoes endocytosis. After being incorporated into an endosomal vesicle, the bond between LDL-LDL receptor is broken, and the LDL receptor is returned to the plasma membrane. Once the LDL-containing endosome merges with a lysosome, lysosomal enzymes break down LDL contents into smaller molecules, including free cholesterol. Free cholesterol is captured by NPC2 and transferred onto NPC1 for efflux from the lysosome. NPC1 then contributes to distribute lysosomal cholesterol among different cellular targets (f.i., the plasma membrane, mitochondria, endoplasmic reticulum), where it will be processed for further use. In addition to LDL-derived cholesterol, lysosomes also process cholesterol from other sources, including damaged organelles or lipid droplets, by merging with autophagosomes. This figure was created using BioRender.com.

Lysosomes are organelles specialized in the breakdown of extra- and intracellular molecules, as well as in the sorting of breakdown products throughout the cell's organelles. To this end, lysosomes contain dozens of enzymes that can break down all classes of macromolecules, from nucleic acids and proteins to lipids and carbohydrates (17). Importantly, lysosomal enzymes are manufactured so that they are active and most efficient at the lysosomal $\mathrm{pH}$, which ranges from 4.5-5.5 and is maintained by a variety of membrane bound ATPconsuming ion pumps. In addition to acting as an intermediate site for incoming nutrients and molecules, phagocytosis-derived microorganisms and foreign antigens captured by 
immune cells are also broken down within lysosomes. Notably, so are intracellular macromolecules and misfolded or aggregated proteins, as well as damaged organelles, such as mitochondria. This process, in which the cell essentially "eats" parts of itself (autophagy), allows cells to maintain homeostasis by removing faulty components, avoiding the accumulation of toxic compounds, and obtaining energy substrates and anabolic components in times of scarcity (18).

Once lysosomal contents are broken down, they must exit lysosomes and reach the cytosol or other organelles, according to cell's needs. In the case of cholesterol, this process is achieved by coordinated action of Niemann-Pick type C2 (NPC2) and NPC1 proteins. As soon as lysosomal acid lipases break down cholesterol esters, the resulting free cholesterol is bound by NPC2, a globular protein in the lysosomal lumen. Subsequently, NPC2 transfers bound cholesterol molecules to NPC1, a lysosomal transmembrane protein (16). While research is ongoing to elucidate the exact mechanisms underlying the interaction between cholesterol, NPC2 and NPC1 and subsequent cholesterol efflux from lysosomes, recent studies give various clues regarding the process. It is likely that cholesterol passes through NPC1 to the outer leaflet of the membrane (17-20). Here, NPC1 acts as membrane contact site tether, thus bringing lysosomes and the plasma membrane or organelles together so that lysosomal free cholesterol is directly incorporated at the target site (21). Of note, lysosomes and NPC1-bound cholesterol are important components of the cellular lipid metabolism network (22), regulating TG and cholesterol storage and de novo synthesis; use of lipids as energy substrates and cellular building blocks and FFA uptake and LDL receptor synthesis and incorporation in the plasma membrane $(21,23-26)$.

Overall, as an integration site of a variety of extra- and intracellular cargo and signals, lysosomes are increasingly acknowledged as important sensors and regulators of cell function, metabolism and homeostasis. Zooming in further, lysosomal NPC proteins are essential for proper lysosomal cholesterol efflux and subsequent cellular use. In addition, recent studies suggest that proper NPC function is key for cholesterol distribution, regulation of lipid metabolism and autophagy $(24,27)$, as well as for the activity of organelles responsible for a multitude of processes, ranging from protein folding to antioxidant defenses. As such, it is unsurprising that disturbances in lysosomal function and NPC have a severe impact in overall cellular homeostasis, as is increasingly observed in a variety of acquired and genetic metabolic disorders, and, even more clearly, in NPC1 disease patients. 


\section{Lysosomal cholesterol accumulation in NPC1 disease and acquired metabolic diseases NPC1 protein dysfunction in disease}

It is estimated that nearly 1 in 100,000 people worldwide are born with NPC1 disease, an autosomal-recessive disorder arising from deleterious mutations in the genes encoding for either NPC1 or NPC2 proteins (accounting for $\sim 95 \%$ and $5 \%$ of cases, respectively) (28). Although rare, NPC1 disease has been the subject of an impressive body of research, greatly due to efforts from clinicians and families to raise awareness and fuel the search for effective diagnostic and therapeutic tools. The range of NPC1 and NPC2 mutations uncovered to date is extensive, and specific mutations can have more or less impact on protein function and disease severity (29). Although many patients experience late-onset and relatively mild symptoms, a significant portion of NPC1 disease patients suffer from debilitating symptoms from a young age and have their life expectancy capped at twenty to thirty years. While the range and severity of clinical manifestations of NPC1 disease are ultimately tied in with the patient's mutation and level of NPC1 protein deficiency, they include: hepatic dysfunction, which often translates into jaundice and hepatomegaly; spleen and lung enlargement and dysfunction; as well as neurological deficits and neuropsychiatric disorders, including motor and cognitive deficits and dysphagia, which take a severe toll in patients. Importantly, although lysosomal cholesterol accumulation is the primary consequence of NPC1 dysfunction, the accompanying oxidative stress and inflammatory responses are important contributors to cell death, organ dysfunction and overall disease burden. Overall, in tandem with progressive disease severity, delayed diagnosis and limited therapeutical options are major contributors to premature mortality rates of NPC1 disease patients.

While there is a wide range of clinical manifestations of NPC1 disease reported to date (30), the liver is commonly affected, culminating in neonatal cholestasis and jaundice, hepatomegaly (i.e., liver enlargement) and liver failure and hepatocarcinoma. As the body's main lipid storage site, it is unsurprising that liver dysfunction is a hallmark of NPC1 disease, and murine models of the disease feature hepatic steatosis, inflammation and fibrosis. Following lysosomal lipid entrapment in the liver, a reduction in subcellular cholesterol levels likely underlies SREBP activation, while defects in oxysterol synthesis dampen LXR-mediated processes, such as ABCA1 production and integration in the plasma membrane. Overall, these events lead to increased cholesterol and FA synthesis, in parallel with defects in excess lipid excretion. Such disturbances of liver-mediated lipid metabolism following NPC1 defects are mirrored by defects in lipoprotein production, namely, by relatively low levels of plasma LDL and HDL in parallel with increased plasma triglyceride levels (31). Furthermore, circulating LDL particles are routinely removed from the circulation by liver macrophages, along with cholesterol-rich apoptotic cells' debris, exacerbating lysosomal lipid accumulation and lipotoxicity in these cells and causing their conversion into foamy cells. Foamy cells, in 
turn, display an activated pro-inflammatory profile which, in the context of chronic dysfunction and oxidative stress, contributes to liver damage that can culminate in fibrosis and acute liver failure. Overall, NPC1 impairment and lysosomal cholesterol entrapment have profound consequences for liver function, which can manifest as severe clinical phenotypes in a significant portion of patients.

While the systemic involvement of NPC1 disease is significant for many patients, the brain is by far the hardest and most commonly hit organ. Although the brain is mostly impermeable to LDL-derived cholesterol, brain cells exhibit high rates of cholesterol biosynthesis and turnover, particularly glial cells, as cholesterol is essential for a myriad of brain functions (32). Oligodendrocytes exhibit the highest rates of cholesterol synthesis in the central nervous system, which they then use for the production of myelin sheath production, a process essential for axon insulation and optimal neurotransmission. Astrocytes, in turn, are prime cholesterol sources for neurons, and astrocyte-derived cholesterol is transported to neurons in ApoE lipoproteins, which, similarly peripheral LDL, undergo endocytosis. In addition to ApoE-derived cholesterol, major sources of lysosomal cholesterol in the brain include myelin sheaths, neurotransmitters, and the plasma membrane. Functionally, cholesterol in the brain is important for a number of processes which are hindered by lysosomal cholesterol entrapment in NPC1 disease, including synapse synthesis and remodelling $(33,34)$; vesiclemediated neurotransmitter transport and recycling and regulation of neurotransmitter and neurotransmitter receptors. In addition, changes in calcium cellular distribution following lysosomal cholesterol accumulation further impact calcium-mediated signalling processes and neurotransmitter signalling transduction (35). Furthermore, due to high energy requirements, the nervous system is particularly prone to excess ROS production, and mitochondrial dysfunction and oxidative stress following lysosomal cholesterol accumulation are major pathological triggers for neurodegeneration in NPC1 disease (36). Overall, the abovementioned mechanisms result in severe neurological symptoms in NPC1 disease, including cognitive and motor deficits, neuropsychiatric disorders, vertical supranuclear gaze palsy and ataxia. Not only are neurological symptoms common among NPC1 disease patients, they are also major contributors to loss of quality of life and reduced life expectancy, and are thus important targets for NPC1 disease therapeutical strategies.

Notably, while full NPC1 impairment, as observed in NPC1 disease, is a relatively rare occurrence, genome-wide association studies have uncovered population-specific NPC1 single nucleotide polymorphism that predispose individuals to overweight, obesity and even cardiovascular disease and type 2 diabetes (25). Furthermore, researchers observed that carriers of rare loss-of-function NPC1 mutations, manifesting as heterozygous deleterious gene mutations, were more predisposed to obesity than control subjects $(37,38)$. While these 
studies are of an associative nature, it is hardly a coincidence that NPC1 variants impacting NPC1 function predispose individuals to metabolic dysfunction. Overall, when taken together with the severe clinical phenotype of NPC1 disease patients, the abovementioned findings emphasize the importance of NPC1 protein and lysosomal function in cell homeostasis and health in general.

\section{The unholy trinity: lysosomal dysfunction, inflammation and oxidative stress}

Following NPC1 impairment, lysosomal cholesterol accumulation compromises lysosomal function in several ways that directly or indirectly contribute to disturbed cell homeostasis. First, lysosomal accumulation of free cholesterol increases the lysosomal pH (39, 40), impairing the activity of lysosomal enzymes and the breakdown of lysosomal contents, which in turn further increases lysosomal engorgement. Furthermore, lysosomal cholesterol buildup disturbs lysosomal iron metabolism and calcium flow (41-43), and impairs endolysosomal traffic and autophagy. In addition, loss of acidic lysosomal $\mathrm{pH}$ contributes to the formation of cholesterol crystals (44), leading to lysosomal membrane destabilization and leakage of lysosomal contents into the cytosol (45). Finally, and possibly quite obviously, lysosomal entrapment of cholesterol compromises its trafficking to the plasma membrane and other organelles, leading to the dysregulation of cellular lipid contents and metabolism. Given the role of lysosomes on a multitude of cellular processes, following lysosomal cholesterol accumulation and lysosomal dysfunction, cell homeostasis and function is inevitably disturbed. In addition, when discussing the link between lysosomal cholesterol accumulation and cell/organ dysfunction (i.e., disease burden), two more mechanisms inextricable from lysosomal dysfunction must be considered: inflammation and oxidative stress.

In case of lysosomal lipid accumulation and associated homeostasis impairment, "distress" pathways are triggered in cells in order to flag abnormalities and encourage repair. Cholesterol crystal formation, as well as impaired autophagy and mitochondrial function following lysosomal cholesterol accumulation $(46,47)$, activates the cells' inflammasome, a family of multiprotein complexes that induce pro-inflammatory responses in response to injury or infection. To date, lysosomal cholesterol overload has been shown to activate NLRP3 inflammasomes, culminating in the maturation of pro-inflammatory IL-1 $\beta$ and IL18 cytokines and in the cell's death via pyroptosis and release of cellular contents in the extracellular space. Upon recognition of apoptotic cells and their contents, as well as exposure to proinflammatory cytokines, immune cells quickly shift towards a pro-inflammatory profile, characterized by increased production of pro-inflammatory cytokines and chemokines that signal for the recruitment and activation of circulating immune cells. Resident and monocytederived macrophages proceed to phagocyte lipid-rich cell debris and toxic compounds, a process that heavily relies on proper lysosomal function. In addition to triggering the 
inflammasome, lysosomal lipid accumulation, for instance due to a dysfunctional NPC1 protein, impairs the ability of macrophages to clear apoptotic cells, thus compromising the resolving of the inflammatory response (48). In addition, lysosomal lipid accumulation in macrophages interferes with excess-lipid packaging into HDL molecules, further exacerbating macrophage dysfunction and activation. Of note, findings of one study suggest that prolonged lysosomal cholesterol accumulation in human macrophages leads to irreversible lysosomal $\mathrm{pH}$ disturbance (39), a factor that may also contribute to sustained inflammatory responses. Finally, lysosomal cholesterol accumulation and impaired lysosomal function can induce senescence in macrophages, as well as interfere with the activation of anti-inflammatory programs within immune cells (49-51). Overall, chronic lysosomal cholesterol accumulation, as in the case of NPC1 disease, results in sustained and stronger pro-inflammatory programming of immune cells which exacerbates tissue damage and disease burden, rather than alleviating it.

Oxidative stress, characterized by excessive ROS production in parallel with compromised antioxidant defenses, goes hand-in-hand with lysosomal lipid accumulation and subsequent impaired homeostasis and inflammatory responses. ROS are a heterogeneous population of molecules whose structures are changed upon losing electrons or accepting electrons from other molecules. Functionally, ROS are inevitable by-products of energy production in mitochondria and metabolic processes in peroxisomes and the endoplasmic reticulum (ER); in addition, ROS are produced in immune cells and play an important role in defense against pathogens. Despite their production being tied-in with healthy physiological processes, excessive ROS production poses a threat to cells, as they can react with molecules such as lipids, proteins and nucleic acids, changing the structure of macromolecules and thus compromising their biological functions. To prevent ROS accumulation and associated damage, cells are equipped with a variety of endogenous antioxidant systems, including superoxide dismutases and glutathione, as well as diet-derived antioxidants such as vitamins E and C.

Lysosomal cholesterol accumulation and subsequent impairment of lysosomal regulation of cellular cholesterol, iron and calcium levels (52), as well as of autophagy (53), predisposes cells to oxidative stress via a variety of mechanisms. First, impaired mitochondria function due to increased cholesterol accumulation increases the production of ROS, while simultaneously compromising the glutathione antioxidant system (54). Secondly, impaired iron and calcium regulation by lysosomes further compromises mitochondria activity and triggers ROS production. Third, impaired autophagy results in improper clearance of dysfunctional mitochondria and ROS, exacerbating oxidative stress. The aforementioned mechanisms underlie increased oxidative stress following lysosomal cholesterol 
accumulation, culminating in the damage and modification of cellular structures and molecules, ROS-mediated inflammasome activation and exacerbation of inflammation and tissue damage. For an overview of the interplay between lysosomal dysfunction, oxidative stress and inflammation, please see Figure 3.

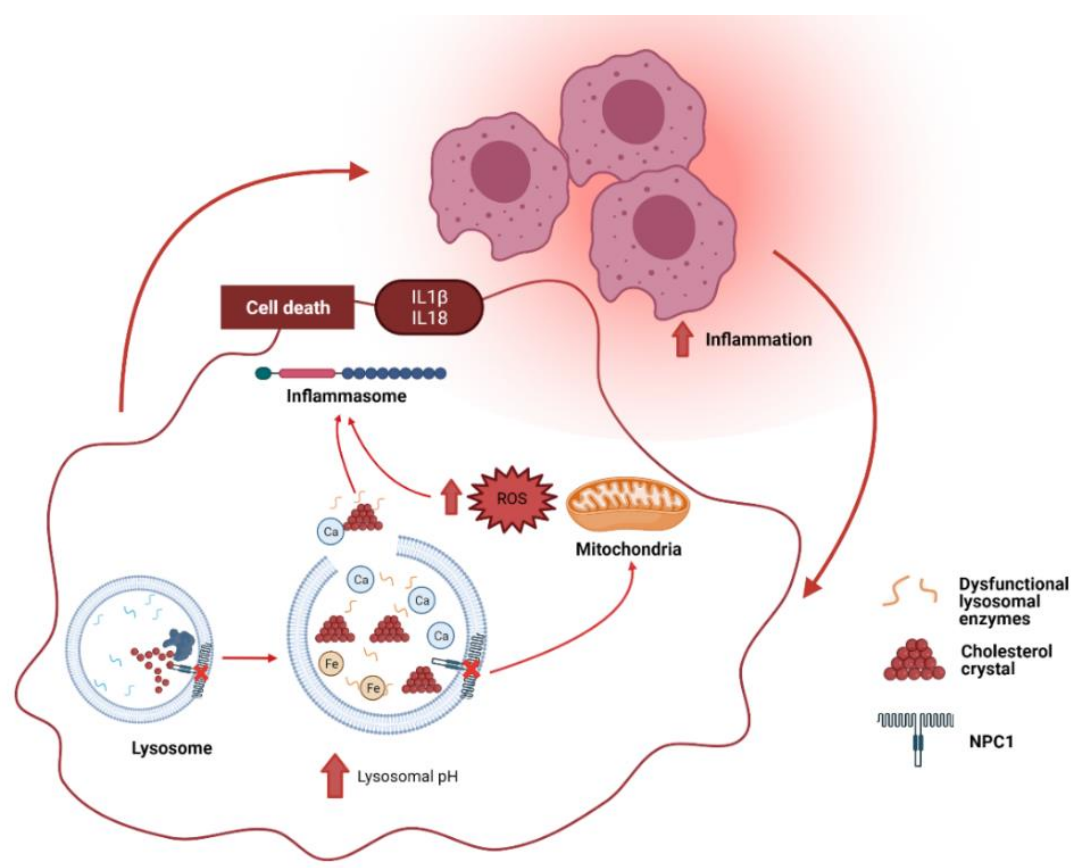

Figure 3 Lysosomal cholesterol accumulation triggers oxidative stress and inflammation. Upon lysosomal cholesterol accumulation, lysosomal $\mathrm{pH}$ increases, impairing the function of lysosomal enzymes and the exchange of calcium and iron, and triggering the formation of cholesterol crystals. These events culminate in overall lysosomal dysfunction and membrane destabilization, resulting in the release of lysosomal contents into the cytosol. Furthermore, lysosomal cholesterol accumulation disturbs cholesterol trafficking to other organelles, including the mitochondria, leading to increased ROS production and oxidative stress. Lysosomal destabilization and oxidative stress, in turn, culminate in cell death and release of pro-inflammatory interleukins via inflammasome activation. Upon recognition of apoptotic cells, immune cells, such as macrophages, switch to a pro-inflammatory profile and proceed to phagocyte the debris. Importantly, lysosomal cholesterol accumulation in immune cells compromises their homeostasis and ability to adopt an anti-inflammatory phenotype. As such, lysosomal cholesterol accumulation triggers and sustains oxidative stress and inflammation, contributing to overall tissue and organ dysfunction. This figure was created using BioRender.com.

\section{Mirroring NPC1-mediated lysosomal dysfunction: oxLDL as a mediator of oxidative stress and inflammation in acquired metabolic diseases}

Finally it is important to emphasize that lysosomal cholesterol accumulation can occur even in individuals with a fully functioning NPC1 protein. In individuals with hypercholesterolemia, due to either excessive lipid consumption and/or glucose/lipid metabolism dysfunction, continuous clearance of excess V- and LDL by macrophages 
culminates in large amounts of lysosomal cholesterol (40). Cholesterol buildup, in turn, can culminate in lysosomal $\mathrm{pH}$ destabilization (44). As abovementioned, improper lysosomal $\mathrm{pH}$ can then trigger the formation of lysosomal cholesterol crystals and impair cholesterol esters hydrolysis, further enlarging lysosomes and compromising lysosomal function, similarly to what is observed in NPC1 disease.

In addition, multiple acquired metabolic diseases, ranging from liver and cardiovascular disease, type 2 diabetes mellitus and cancer, are characterized by oxidative stress (55), which can further trigger/exacerbate lysosomal lipid accumulation via oxidative lipid modifications. Of note, LDL contents are particularly prone to oxidative modifications, which convert LDL to oxidized LDL (oxLDL). OxLDL is increasingly acknowledged as an important mediator in highly prevalent diseases characterized by lysosomal and lipid dysfunction, oxidative stress and inflammation, such as the abovementioned (56-59). To date, a wide range of oxLDL molecules have been described in vitro and in vivo, depending on the extent and molecular targets (cholesterol esters, apolipoproteins) of oxidative LDL modifications (58). While the exact mechanisms underlying increased oxLDL production in humans is not fully elucidated, a team of researchers has shown that lysosomal LDL oxidation can occur in lipid-overloaded macrophages via iron and iron-metabolism related enzymes (60). Importantly, oxidized LDL contents, such as cholesterol esters, do not undergo hydrolysis by lysosomal acid lipases as non-oxidized contents do (40), and thus become entrapped in lysosomes (61). In addition, prolonged lysosomal oxLDL entrapment further compromises the hydrolysis of non-oxidized LDL contents (40), exacerbating lysosomal cholesterol accumulation (62).

In addition to triggering lysosomal cholesterol accumulation and, subsequently, lysosomal dysfunction, circulating oxLDL can directly trigger pro-inflammatory responses in immune cells. Unlike native LDL, oxLDL is unable to bind to cellular LDL receptors; instead, it interacts with scavenger receptors, Toll-like receptors and lectin-type oxidized LDL receptors (58). The binding of oxLDL to the aforementioned cellular receptors initiates proinflammatory and apoptotic cascades within cells, contributing to inflammation and oxidative stress in parallel with lysosomal cholesterol accumulation-derived effects on cell homeostasis. Thus, increased oxLDL production and uptake by cells in metabolic diseases induces lysosomal dysfunction that mirrors NPC1-mediated damage in NPC1 disease and results in increased disease burden. Furthermore, elevated plasma oxLDL levels have been reported in NPC1 disease patients compared to healthy controls (63). In addition to strengthening the relationship between lysosomal cholesterol build up, oxidative stress and oxLDL formation, these findings also emphasize the role of oxLDL as a mediator of severe metabolic disease burden. For an overview of mechanisms underlying the impact of oxLDL on disease burden, please refer to Figure 4. 


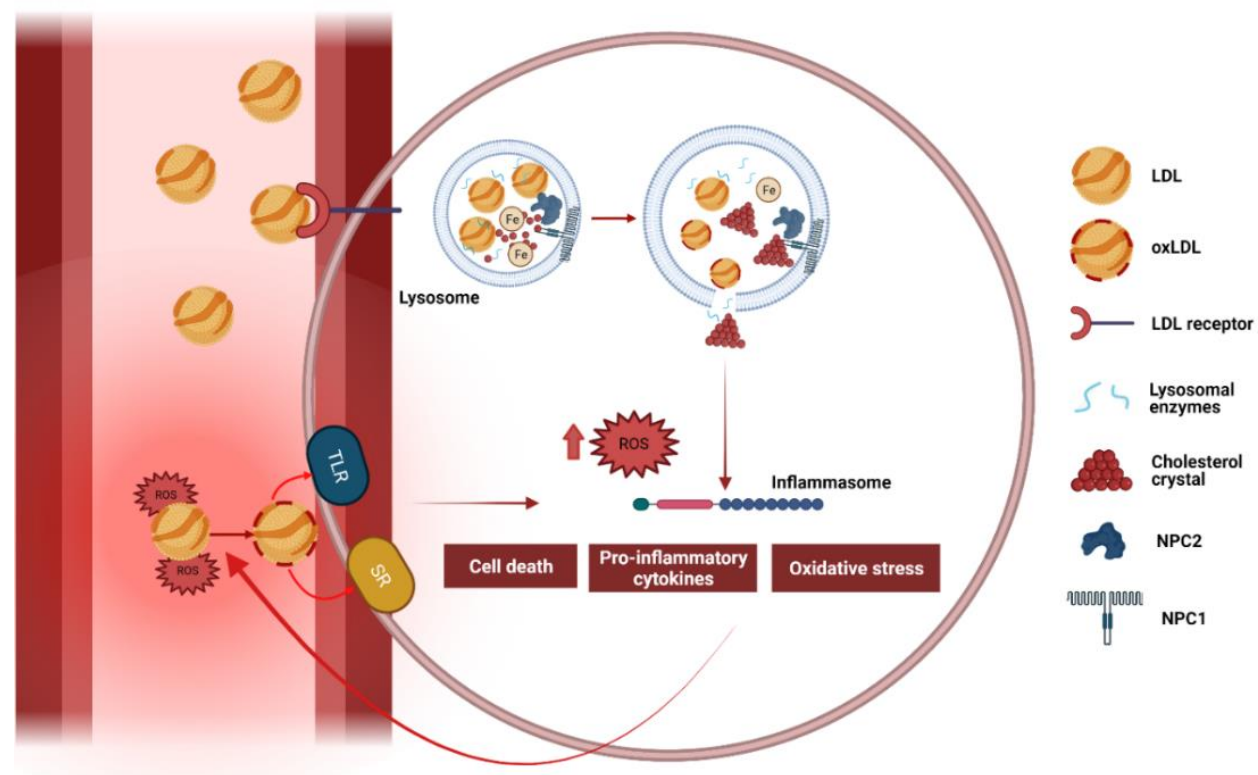

Figure 4 OxLDL-driven vicious cycle: lysosomal dysfunction, oxidative stress and inflammation. In individuals with oxidative stress, circulating LDL is converted to oxLDL, which, unlike native LDL, binds to receptors such as toll-like receptors (TLR) and scavenger receptors (SR). In addition, disturbances in lysosomal iron metabolism following lysosomal cholesterol overload can also oxidize lysosomal LDL, causing further lysosomal dysregulation and leakage of lysosomal contents. Consequently, the inflammasome is activated, triggering cell death and the release of proinflammatory cytokines and ROS. Increasing ROS and oxLDL-rich debris further exacerbates oxidative stress and inflammation, and exacerbates the formation of oxLDL, thus forming a pathological vicious cycle. This figure was created using BioRender.com.

Overall, lysosomal lipid accumulation is increasingly acknowledged as having severe consequences for cellular metabolism and homeostasis, with far-reaching consequences that can trigger and/or exacerbate the burden of diseases ranging from highly prevalent acquired metabolic diseases and NPC1 disease. As such, further research into lysosomal function and modulation strategies may yield promising results in the development of widely applicable therapeutic tools. 


\section{Aims and outline of this thesis}

A growing body of research pinpoints lysosomal lipid accumulation and NPC1 impairment as an important contributor to metabolic dysfunction, oxidative stress and inflammation in a wide array of diseases (NPC1 disease, non-alcoholic steatohepatitis (NASH), atherosclerosis and cancer). As such, further analyzing the impact of NPC1 and lysosomal dysfunction in metabolic diseases may yield better understanding of pathophysiological mechanisms and the development of new therapeutic tools.

In chapter 2 of this thesis, we evaluated the impact of dietary plant stanol supplementation on systemic features of a mouse model for NPC1 disease. Subsequently, chapter 3 aimed to assess the relationship between dietary cholesterol and anti-inflammatory effects of plant stanol supplementation in a mouse model for metabolic inflammation characterized by hematopoietic NPC1 dysfunction. In chapter 4, we explored the influence of hematopoietic NPC1 dysfunction on the gut microbiome in the context of metabolic inflammation. In chapter 5, we analyzed the impact of pneumococcal immunization on antibody levels against oxLDL and on systemic and neurological features of NPC1 disease. Furthermore, to establish whether a pneumococcal vaccine increases antibodies against oxLDL in humans, in chapter 6 we conducted a pilot clinical trial in which patients with familial partial lipodystrophy, familial hypercholesterolemia and Niemann-Pick type B disease received a pneumococcal vaccine. Finally, the findings and impact of this thesis are discussed in chapter 7 , in the context of acquired and genetic metabolic diseases. 


\section{REFERENCES}

1. Blüher M. Obesity: global epidemiology and pathogenesis. Nature Reviews Endocrinology. 2019;15(5):288-98.

2. WHO. Noncommunicable diseases: Key Facts 2018.Last updated on: 13/04/2021. Available from: https://www.who.int/news-room/fact-sheets/detail/noncommunicablediseases. Accessed on: 05/05/2021

3. Feher J. 8.5 - Digestion and Absorption of the Macronutrients. In: Feher J, editor. Quantitative Human Physiology (Second Edition). Boston: Academic Press; 2017. p. 821-33.

4. Singh H, Ye A, Horne D. Structuring food emulsions in the gastrointestinal tract to modify lipid digestion. Progress in Lipid Research. 2009;48(2):92-100.

5. Chiang J. Liver Physiology: Metabolism and Detoxification. In: McManus LM, Mitchell RN, editors. Pathobiology of Human Disease. San Diego: Academic Press; 2014. p. 1770-82.

6. Trefts E, Gannon M, Wasserman DH. The liver. Current Biology. 2017;27(21):R1147R51.

7. Kmieć Z. Cooperation of liver cells in health and disease. Advances in anatomy, embryology, and cell biology. 2001;161:Iii-xiii, 1-151.

8. $\quad$ Rui L. Energy metabolism in the liver. Compr Physiol. 2014;4(1):177-97.

9. Liu X, Wang H, Liang X, Roberts MS. Chapter 30 - Hepatic Metabolism in Liver Health and Disease. In: Muriel P, editor. Liver Pathophysiology. Boston: Academic Press; 2017. p. 391-400.

10. Kwiterovich PO. The metabolic pathways of high-density lipoprotein, low-density lipoprotein, and triglycerides: a current review. The American Journal of Cardiology. 2000;86(12, Supplement 1):5-10.

11. Björkhem I, Meaney S. Brain Cholesterol: Long Secret Life Behind a Barrier. Arterioscler Thromb Vasc Biol. 2004;24(5):806-15.

12. Pfrieger FW, Ungerer N. Cholesterol metabolism in neurons and astrocytes. Progress in Lipid Research. 2011;50(4):357-71.

13. Babiker A, Diczfalusy U. Transport of side-chain oxidized oxysterols in the human circulation. Biochimica et Biophysica Acta (BBA) - Lipids and Lipid Metabolism. 1998;1392(2):333-9.

14. Knight R, Callewaert C, Marotz C, Hyde ER, Debelius JW, McDonald D, et al. The Microbiome and Human Biology. Annual Review of Genomics and Human Genetics. 2017;18(1):65-86.

15. Wang B, Yao M, Lv L, Ling Z, Li L. The Human Microbiota in Health and Disease. Engineering. 2017;3(1):71-82. 
16. Maxfield FR. Role of Lysosomes in Lipid Metabolism. Lysosomes: Biology, Diseases, and Therapeutics2016. p. 87-100.

17. Pfeffer SR. Clues to NPC1-mediated cholesterol export from lysosomes. Proc Natl Acad Sci U S A. 2016;113(29):7941-3.

18. Pfeffer SR. NPC intracellular cholesterol transporter 1 (NPC1)-mediated cholesterol export from lysosomes. Journal of Biological Chemistry. 2019;294(5):1706-9.

19. Qian H, Wu X, Du X, Yao X, Zhao X, Lee J, et al. Structural Basis of Low-pHDependent Lysosomal Cholesterol Egress by NPC1 and NPC2. Cell. 2020;182(1):98-111.e18.

20. Li X, Saha P, Li J, Blobel G, Pfeffer SR. Clues to the mechanism of cholesterol transfer from the structure of NPC1 middle lumenal domain bound to NPC2. Proc Natl Acad Sci U S A. 2016;113(36):10079-84.

21. Höglinger D, Burgoyne T, Sanchez-Heras E, Hartwig P, Colaco A, Newton J, et al. NPC1 regulates ER contacts with endocytic organelles to mediate cholesterol egress. Nature Communications. 2019;10(1):4276.

22. Meng Y, Heybrock S, Neculai D, Saftig P. Cholesterol Handling in Lysosomes and Beyond. Trends in Cell Biology. 2020;30(6):452-66.

23. Bartel K, Pein H, Popper B, Schmitt S, Janaki-Raman S, Schulze A, et al. Connecting lysosomes and mitochondria - a novel role for lipid metabolism in cancer cell death. Cell Communication and Signaling. 2019;17(1):87.

24. Davis OB, Shin HR, Lim C-Y, Wu EY, Kukurugya M, Maher CF, et al. NPC1mTORC1 Signaling Couples Cholesterol Sensing to Organelle Homeostasis and Is a Targetable Pathway in Niemann-Pick Type C. Developmental Cell. 2021;56(3):260-76.e7.

25. Lamri A, Pigeyre M, Garver WS, Meyre D. The Extending Spectrum of NPC1Related Human Disorders: From Niemann-Pick C1 Disease to Obesity. Endocrine Reviews. 2018;39(2):192-220.

26. Li F, Zhang H. Lysosomal Acid Lipase in Lipid Metabolism and Beyond. Arterioscler Thromb Vasc Biol. 2019;39(5):850-6.

27. Jaishy B, Abel ED. Lipids, lysosomes, and autophagy. Journal of Lipid Research. 2016;57(9):1619-35.

28. Geberhiwot T, Moro A, Dardis A, Ramaswami U, Sirrs S, Marfa MP, et al. Consensus clinical management guidelines for Niemann-Pick disease type C. Orphanet Journal of Rare Diseases. 2018;13(1):50.

29. Millat G, Marçais C, Tomasetto C, Chikh K, Fensom AH, Harzer K, et al. NiemannPick C1 Disease: Correlations between NPC1 Mutations, Levels of NPC1 Protein, and Phenotypes Emphasize the Functional Significance of the Putative Sterol-Sensing Domain and of the Cysteine-Rich Luminal Loop. American Journal of Human Genetics. 2001;68(6):1373-85. 
30. Bräuer AU, Kuhla A, Holzmann C, Wree A, Witt M. Current Challenges in Understanding the Cellular and Molecular Mechanisms in Niemann-Pick Disease Type C1. Int J Mol Sci. 2019;20(18):4392.

31. Garver WS, Jelinek D, Meaney FJ, Flynn J, Pettit KM, Shepherd G, et al. The National Niemann-Pick Type C1 Disease Database: correlation of lipid profiles, mutations, and biochemical phenotypes. Journal of Lipid Research. 2010;51(2):406-15.

32. Arenas F, Garcia-Ruiz C, Fernandez-Checa JC. Intracellular Cholesterol Trafficking and Impact in Neurodegeneration. Frontiers in Molecular Neuroscience. 2017;10:382.

33. Yoshii A, Constantine-Paton M. Postsynaptic BDNF-TrkB signaling in synapse maturation, plasticity, and disease. Developmental Neurobiology. 2010;70(5):304-22.

34. Suzuki S, Numakawa T, Shimazu K, Koshimizu H, Hara T, Hatanaka H, et al. BDNFinduced recruitment of TrkB receptor into neuronal lipid rafts : roles in synaptic modulation. Journal of Cell Biology. 2004;167(6):1205-15.

35. Vivas O, Tiscione SA, Dixon RE, Ory DS, Dickson EJ. Niemann-Pick Type C Disease Reveals a Link between Lysosomal Cholesterol and PtdIns(4,5)P2 That Regulates Neuronal Excitability. Cell Reports. 2019;27(9):2636-48.e4.

36. Torres S, Matías N, Baulies A, Nuñez S, Alarcon-Vila C, Martinez L, et al. Mitochondrial GSH replenishment as a potential therapeutic approach for Niemann Pick type C disease. Redox Biology. 2017;11:60-72.

37. Liu R, Zou Y, Hong J, Cao M, Cui B, Zhang H, et al. Rare Loss-of-Function Variants in \&lt;em\&gt;NPC1\&lt;/em\&gt; Predispose to Human Obesity. Diabetes. 2017;66(4):935.

38. Chiorean A, Garver WS, Meyre D. Signatures of natural selection and ethnic-specific prevalence of NPC1 pathogenic mutations contributing to obesity and Niemann-Pick disease type C1. Scientific Reports. 2020;10(1):18787.

39. Cox BE, Griffin EE, Ullery JC, W. Gray J. Effects of cellular cholesterol loading on macrophage foam cell lysosome acidification $<$ sup $>$ s\& $\# x 20 \mathrm{de} ;</$ sup $>$. Journal of Lipid Research. 2007;48(5):1012-21.

40. Jerome WG, Cox BE, Griffin EE, Ullery JC. Lysosomal cholesterol accumulation inhibits subsequent hydrolysis of lipoprotein cholesteryl ester. Microsc Microanal. 2008;14(2):138-49.

41. Shen D, Wang X, Li X, Zhang X, Yao Z, Dibble S, et al. Lipid storage disorders block lysosomal trafficking by inhibiting a TRP channel and lysosomal calcium release. Nature Communications. 2012;3(1):731.

42. Yambire KF, Rostosky C, Watanabe T, Pacheu-Grau D, Torres-Odio S, SanchezGuerrero A, et al. Impaired lysosomal acidification triggers iron deficiency and inflammation in vivo. Elife. 2019;8:e51031. 
43. Gómez NM, Lu W, Lim JC, Kiselyov K, Campagno KE, Grishchuk Y, et al. Robust lysosomal calcium signaling through channel TRPML1 is impaired by lysosomal lipid accumulation. The FASEB Journal. 2018;32(2):782-94.

44. Baumer Y, Dey AK, Gutierrez-Huerta CA, Khalil NO, Sekine Y, Sanda GE, et al. Hyperlipidaemia and IFNgamma/TNFalpha Synergism are associated with cholesterol crystal formation in Endothelial cells partly through modulation of Lysosomal $\mathrm{pH}$ and Cholesterol homeostasis. EBioMedicine. 2020;59.

45. Sergin I, Evans TD, Razani B. Degradation and beyond: the macrophage lysosome as a nexus for nutrient sensing and processing in atherosclerosis. Curr Opin Lipidol. 2015;26(5):394-404.

46. Luo X, Li Y, Yang P, Chen Y, Wei L, Yu T, et al. Obesity induces preadipocyte CD36 expression promoting inflammation via the disruption of lysosomal calcium homeostasis and lysosome function. EBioMedicine. 2020;56:102797.

47. Rajamäki K, Lappalainen J, Öörni K, Välimäki E, Matikainen S, Kovanen PT, et al. Cholesterol Crystals Activate the NLRP3 Inflammasome in Human Macrophages: A Novel Link between Cholesterol Metabolism and Inflammation. PLOS ONE. 2010;5(7):e11765.

48. Viaud M, Ivanov S, Vujic N, Duta-Mare M, Aira L-E, Barouillet T, et al. Lysosomal Cholesterol Hydrolysis Couples Efferocytosis to Anti-Inflammatory Oxysterol Production. Circulation Research. 2018;122(10):1369-84.

49. Vion A-C, Kheloufi M, Hammoutene A, Poisson J, Lasselin J, Devue C, et al. Autophagy is required for endothelial cell alignment and atheroprotection under physiological blood flow. Proceedings of the National Academy of Sciences. 2017;114(41):E8675.

50. Huang SC-C, Everts B, Ivanova Y, O'Sullivan D, Nascimento M, Smith AM, et al. Cell-intrinsic lysosomal lipolysis is essential for alternative activation of macrophages. Nature Immunology. 2014;15(9):846-55.

51. Deretic V. Autophagy in inflammation, infection, and immunometabolism. Immunity. 2021;54(3):437-53.

52. Peng W, Wong YC, Krainc D. Mitochondria-lysosome contacts regulate mitochondrial Ca\&lt;sup\&gt;2+\&lt;/sup\&gt; dynamics via lysosomal TRPML1. Proceedings of the National Academy of Sciences. 2020;117(32):19266.

53. Zhang X, Cheng X, Yu L, Yang J, Calvo R, Patnaik S, et al. MCOLN1 is a ROS sensor in lysosomes that regulates autophagy. Nature Communications. 2016;7(1):12109.

54. Torres S, Balboa E, Zanlungo S, Enrich C, Garcia-Ruiz C, Fernandez-Checa JC. Lysosomal and Mitochondrial Liaisons in Niemann-Pick Disease. 2017;8(982).

55. Rani V, Deep G, Singh RK, Palle K, Yadav UCS. Oxidative stress and metabolic disorders: Pathogenesis and therapeutic strategies. Life Sciences. 2016;148:183-93. 
56. Matsuura E, Hughes GRV, Khamashta MA. Oxidation of LDL and its clinical implication. Autoimmunity Reviews. 2008;7(7):558-66.

57. Omar NN, El Hefnawy MH, El Soda MF, Heider NM, Hamed HI. Assessment of oxLDL, anti-oxLDL antibodies and lipoprotein-associated phospholipase A2 as cardiovascular risk markers in obese adolescents with and without T1DM. Bulletin of Faculty of Pharmacy, Cairo University. 2017;55(2):325-31.

58. Levitan I, Volkov S, Subbaiah PV. Oxidized LDL: diversity, patterns of recognition, and pathophysiology. Antioxid Redox Signal. 2010;13(1):39-75.

59. Hurtado-Roca Y, Bueno H, Fernandez-Ortiz A, Ordovas JM, Ibañez B, Fuster V, et al. Oxidized LDL Is Associated With Metabolic Syndrome Traits Independently of Central Obesity and Insulin Resistance. Diabetes. 2017;66(2):474.

60. Ojo OO, Ahmad F, Leake DS. 194 Ferritin Oxidises Low Density Lipoprotein at Lysosomal PH. Heart. 2016;102(Suppl 6):A131.

61. Yancey PG, Jerome WG. Lysosomal cholesterol derived from mildly oxidized low density lipoprotein is resistant to efflux. Journal of Lipid Research. 2001;42(3):317-27.

62. Ahmad F, Leake DS. Lysosomal oxidation of LDL alters lysosomal $\mathrm{pH}$, induces senescence, and increases secretion of pro-inflammatory cytokines in human macrophages [S]. Journal of Lipid Research. 2019;60(1):98-110.

63. Porter FD, Scherrer DE, Lanier MH, Langmade SJ, Molugu V, Gale SE, et al. Cholesterol oxidation products are sensitive and specific blood-based biomarkers for Niemann-Pick C1 disease. Sci Transl Med. 2010;2(56):56ra81-56ra81. 



\section{Chapter 2}

Dietary plant stanol ester supplementation reduces peripheral symptoms in a mouse model of Niemann-Pick type $\mathrm{C} 1$ disease.

Inês Magro dos Reis, Tom Houben, Yvonne Oligschläger, Leoni Bücken, Hellen Steinbusch, David Cassiman, Dieter Lütjohann, Marit Westerterp, Jos Prickaerts, Jogchum Plat, Ronit Shiri-Sverdlov

Journal of Lipid Research, 2020; 61 (6), 830-839 


\begin{abstract}
Niemann-Pick type C1 (NPC1) disease is a rare genetic condition in which the function of the lysosomal cholesterol transporter NPC1 protein is impaired. Consequently, sphingolipids and cholesterol accumulate in lysosomes of all tissues, triggering a cascade of pathological events that culminate in severe systemic and neurological symptoms. Lysosomal cholesterol accumulation is also a key-factor in the development of atherosclerosis and non-alcoholic steatohepatitis (NASH). In these two metabolic diseases, the administration of plant stanol esters has been shown to ameliorate cellular cholesterol accumulation and inflammation. Given the overlap of pathological mechanisms among atherosclerosis, NASH and NPC1 disease, we sought to investigate whether dietary supplementation with plant stanol esters improves the peripheral features of NPC1 disease. To this end, we used an NPC1 murine model featuring an $N p c 1$ null allele $\left(N p c 1^{\text {nih }}\right)$, creating a dysfunctional NPC1 protein. $N p c 1^{\text {nih }}$ mice were fed a two or six percent plant stanol esters-enriched diet over the course of 5 weeks. During this period, hepatic and blood lipid and inflammatory profiles were assessed. $N p c 1^{\text {nih }}$ mice fed the plant stanol-enriched diet exhibited lower hepatic cholesterol accumulation, damage and inflammation than regular chow-fed $N p c 1^{\text {nih }}$ mice. Moreover, plant stanol consumption shifted circulating T-cells and monocytes in particular towards an antiinflammatory profile. Overall, these effects were stronger following dietary supplementation with $6 \%$ stanols, suggesting a dose-dependent effect. The findings of our study highlight the potential use of plant stanols as an affordable complementary means to ameliorate disorders in hepatic and blood lipid metabolism and reduce inflammation in NPC1 disease.
\end{abstract}




\section{INTRODUCTION}

Niemann-Pick type C (NPC) disease is a rare lysosomal storage disorder caused by deleterious mutations in NPC1 or NPC2. It is estimated that NPC disease affects one in 100,000 live births, with mutations in NPC1 occurring in approximately $95 \%$ of cases (1). Although caused by different genetic mutations, NPC1 and NPC2 diseases are clinically indistinguishable, as both NPC1 and NPC2 proteins are required for endolysosomal cholesterol efflux. Upon endocytosis, low-density lipoproteins (LDL) merge with late endosomes/lysosomes (LEL), where lysosomal acid lipases hydrolyze LDL-derived cholesteryl esters. NPC2, a protein located on the luminal surface of LEL, binds the resulting free cholesterol and directs it to the luminal domain of NPC1, a LEL transmembrane protein. Studies indicate that the NPC1 protein subsequently delivers the free cholesterol to the plasma membrane and to the endoplasmic reticulum, possibly via membrane contact sites between the endoplasmic reticulum and LELs $(2,3)$. Due to compromised NPC1 protein function, NPC1 disease (OMIM \#257220) is characterized by endolysosomal cholesterol and sphingolipid accumulation in all cells.(4). The age of onset and clinical features of NPC1 disease are heterogeneous, likely because of the variability of NPC1 mutations among patients $(5,6)$. Nonetheless, the nervous system of NPC1 disease patients is commonly severely affected, triggering the development of neuropsychiatric disorders and cognitive and motor function degeneration (6). In addition, systemic dysfunctions such as jaundice, cholestatic disease, hepatosplenomegaly, liver and pulmonary disease occur in a significant amount of patients. While such systemic symptoms are most severe in the perinatal and infantile stage of the disease, and tend to become stable in older NPC1 disease patients, in some cases peripheral dysfunction can further progress and result in cirrhosis and hepatocellular carcinoma (6-9). Although awareness of NPC1 disease has increased in recent years, early diagnosis and curative treatments are still lacking. Miglustat, a sphingolipid synthesis inhibitor, was approved in the European Union in 2009 as the first NPC1 disease-targeted drug (10). While Miglustat has been shown to reduce the progression of neurological deterioration in NPC1 disease patients, a report indicates it has a minor impact on systemic symptoms such as splenomegaly (11). Furthermore, intrathecal administration of 2hydroxypropyl- $\beta$-cyclodextrin to reduce neurological symptom progression in NPC1 disease is currently being evaluated in phase 2/3 clinical trials (ClinicalTrials.gov Identifier: NCT02534844) (12). Despite promising results, the use of 2-hydroxypropyl- $\beta$-cyclodextrin as a therapeutical compound in NPC1 disease faces several challenges, including the administration route and side-effects (12). Finally, a different clinical study is currently evaluating the effects of intravenous 2-hydroxypropyl- $\beta$-cyclodextrin administration on hepatic NPC1 disease symptoms (ClinicalTrials.gov Identifier: NCT03887533). Overall, considering the limited amount and scope of NPC1 disease treatments, further research is 
needed to develop a wider range of interventions that can modify NPC1 disease progression $(6,13)$.

Lysosomal lipid accumulation is at the core of NPC1 disease pathology and triggers a series of events that culminate in tissue and organ dysfunction. Such events include disturbed lysosomal function and lipid metabolism, as well as increased oxidative stress, inflammation and apoptosis (14-17). The aforementioned pathological mechanisms mirror those observed, though to a lesser extent, in atherosclerosis and non-alcoholic steatohepatitis (NASH). Similarly to NPC1 disease, these metabolic disorders are characterized by lysosomal lipid accumulation in macrophages, which has been shown to be a key factor in disease severity and development (18-22).

Notably, in vitro and in vivo studies have shown plant stanol ester supplementation to be beneficial in both NASH and atherosclerosis $(23,24)$. Plant stanols are essential components of plant cells derived from the saturation of plant sterols, which share a similar chemical structure and biochemical functions as the mammalian cholesterol (25). The average human daily intake of plant stanols is $20-50 \mathrm{mg}$, of which up to $0.15 \%$ is estimated to effectively be absorbed in the small intestine $(26,27)$. Dietary supplementation with plant stanol esters has well-known plasma cholesterol-lowering effects, presumably since they interfere with intestinal cholesterol absorption (27-29). Specifically, dietary plant stanol supplementation has been shown to reduce cellular cholesterol accumulation in NASH and atherosclerosis models. In addition, the aforementioned studies indicate that plant stanol esters supplementation ameliorates hepatic inflammation, a mechanism that also contributes to NPC1 disease severity $(23,30,31)$.

Considering the parallels between the pathological mechanisms of NPC1 disease, atherosclerosis and NASH, the aim of this study was to investigate whether dietary supplementation with plant stanol esters also improves peripheral features in NPC1 disease. To this end, we used an NPC1 disease murine model which expresses an Npc1 allele with a frameshift mutation ( $\left.N p c 1^{n i h}\right)$ that results in the loss of function of the corresponding NPC1 protein (13). While the $N p c 1^{\text {nih }}$ allele was originally discovered and maintained in the BALB/C mouse strain, here we used $N p c 1^{\text {nih }}$ mice with a C57BL/6 genetic background, a model which has been previously described and which results in a more severe NPC1 peripheral disease phenotype (32). To investigate our hypothesis, two weeks-old $N p c 1^{\text {in }}$ mice received a normal chow or a two or six percent plant stanol esters-enriched chow diet for five weeks. $N p c 1^{w t}$ mice fed a regular chow were included as a control group for NPC1 disease phenotype. Npc1 ${ }^{\text {nih }}$ mice fed a plant stanol-enriched diet showed decreased hepatic cholesterol accumulation, as well as reduced hepatic damage and inflammation. In addition to the localized effects in the 
liver, plant stanol administration led to a systemic immune shift towards an antiinflammatory profile, as assessed by FACS analysis of white blood cells. Of note, the effect of plant stanol esters on peripheral NPC1 disease symptoms was overall more pronounced after supplementation of six percent plant stanol-enriched diet compared with two percent enriched diet, proving the beneficial effect of plant stanols to be dose-dependent.

Overall, these findings highlight the potential of plant stanol esters as a widely available and affordable additional tool to ameliorate hepatic symptoms and the phenotype of blood monocytes and T-cells in NPC1 disease patients, in combination with other therapies, such as Miglustat and 2-hydroxypropyl- $\beta$-cyclodextrin. 


\section{MATERIALS AND METHODS}

Mice

Male and female $N p c 1^{\text {nih }}$ mice were derived from heterozygous founders (C57BL/6 / Npc1 ${ }^{\text {nih }}$ ). Given the reduced lifespan of $N p c 1^{\text {nilh }}$ mice, as soon as the genotypes of the mice were known, the experimental diets were administered to the mothers, who would transfer the experimental diet to the pups via breastmilk (week 0 of the experiment). After the weaning period, at 14 days of age, mice began being fed the appropriate diet as solid chow. 13 and 16 $N p c 1^{\text {nih }}$ mice received a two percent and six percent plant stanol esters-enriched diet, respectively (manufactured by Arie Blok B.V., Woerden, The Netherlands). Npc1 $1^{\text {wt }}$ and Npc1 ${ }^{\text {nih }}$ mice fed a regular chow $\operatorname{diet}$ ( $\mathrm{n}=10$ and 13 , respectively) were included as controls. Mice were housed under standard conditions and given free access to food and water. For an overview of the study setup and dietary plant stanol and sterol composition, please refer to Fig. S1 and S2, respectively. Blood from the tail vein was collected on weeks 3 and 5 of the experiment, when mice were 35 and 49 days old, respectively. All tissues were isolated and snap-frozen in liquid nitrogen and stored at $-80^{\circ} \mathrm{C}$ or fixed in $4 \%$ formaldehyde/PBS. The collection of blood and tissue specimens, biochemical determination of lipids in plasma and liver, RNA isolation, cDNA synthesis and qPCR were performed as described previously (33-35). All experiments were performed according to Dutch laws and approved by the Animal Experiment Committee of Maastricht University.

\section{GC-MS}

Plant sterols (sitosterol, campesterol) and plant stanols (sitostanol, campestanol) content in food was analyzed by gas-liquid chromatography-mass spectroscopy (GC-MS) as described previously (36).

\section{Genotyping}

Genotypes of animals were determined by PCR analysis of tail DNA. Toes were clipped at postnatal day 2 and homogenized in DirectPCR-Tail (Peqlab, Erlangen, Germany) supplemented with a tenth part Proteinase K (Qiagen, Hilden, Germany). Three hours of incubation at $56^{\circ} \mathrm{C}$ and agitation at 1000 rounds per minute on a Thermo Mixer were followed by $45 \mathrm{~min}$ of heating at $85^{\circ} \mathrm{C}$ to inactivate the proteinase. Samples were then spun at full speed in a benchtop centrifuge for $1 \mathrm{~min}$. The PCR reactions were performed with $0.5 \mathrm{ml}$ of the obtained extracts. Each lysate underwent two PCRs. Primers gccaagtaggcgacgact and catctactgggtctccatatgtat identified the wild-type allele and primers gccaagtaggcgacgact and ttccaattgtgatctttccaa identified the mutant allele. Both PCRs were carried out under the same cycling conditions. 


\section{Alanine aminotransferase (ALT) measurements}

Plasma ALT levels were measured with the Reflotron ${ }^{\circledR}$ test strips (Roche, Germany) according to manufacturer's instructions, using the Reflotron ${ }^{\circledR}$ apparatus.

\section{Immunohistochemistry}

Frozen liver sections $(7 \mu \mathrm{m})$ were fixed in acetone and blocked for endogenous peroxidase by incubation with $0.25 \%$ of $0.03 \% \mathrm{H}_{2} \mathrm{O}_{2}$ for 5 minutes. Primary antibodies used were against hepatic macrophages (rat anti-mouse CD68, clone FA11) and infiltrated macrophages and neutrophils (rat anti-mouse Mac-1 [M1/70]). 3-Amino-9-ethylcarbazole (AEC) was applied as color substrate and hematoxylin for nuclear counterstain. Sections were enclosed with Faramount aqueous mounting medium. Pictures were taken with a Nikon digital camera DMX1200 and ACT-1 v2.63 software (Nikon Instruments Europe, Amstelveen, The Netherlands). Infiltrated macrophages and neutrophil cells (Mac-1) were counted by two blinded researchers in six microscopical views (original magnification, 200x) and were indicated as number of cells per square millimeter (cells/mm2). Immunostainings for hepatic macrophages (CD68) were evaluated by an experienced pathologist and given a score in arbitrary units (A.U.).

\section{Plasma FACS analyses}

Tail vein blood was collected from $N p c 1^{w t}$ and $N p c 1^{\text {nih }}$ mice on weeks 3 and 5 of the experiment, when mice were 35 and 49 days old, respectively. Stainings were performed using Trucount tubes (BD Biosciences, Breda, The Netherlands), according to the manufacturer's instructions, to detect the following populations: monocytes (NK1.1-Ly6GCD11b+; Ly6C) and T-cells (CD3+; CD4+; CD8+). Briefly, heparinized blood samples were mixed and incubated for 10 minutes in the dark at room temperature (RT) with CD16/32 antibody (eBioscience, Halle-Zoersel, Belgium) to block Fc receptor. Samples were then gently vortexed with the appropriate antibodies and incubated in the dark at RT for 20 minutes. All antibodies were diluted in FACS buffer (PBS, 0.1\% BSA, 0.01\% sodium azide). In this study, the following antibodies were used: PE Mouse Anti-Mouse NK-1.1 (1:100); APC-Cy ${ }^{\mathrm{TM}}$ R Rat Anti-Mouse Ly-6G (1:100); PE-Cy ${ }^{\mathrm{TM} 7}$ Rat Anti-CD11b (1:300); APC-H7 Rat anti-Mouse CD4 (1:100) (BD, San Jose, USA); CD3 Monoclonal Antibody (1:100), CD8a Monoclonal Antibody (1:50) (eBioscience ${ }^{\mathrm{TM}}$ from Thermo Fisher Scientific, San Diego, USA); Anti-Mouse Ly-6CAPC (1:10) (Miltenyi, Bergisch Gladbach, Germany). Finally, samples were mixed and incubated in the dark at RT for 15 minutes with an erylysis solution $(8.4 \mathrm{~g} \mathrm{NH} 4 \mathrm{CL}+0.84 \mathrm{~g}$ $\mathrm{NAHCO}_{3}$ in 1 liter $\mathrm{H}_{2} \mathrm{O}$, pH 7.2-7.4). Sample stainings were quantified within 1 hour using BD FACSCanto II flow cytometer (BD Biosciences). 
Data are expressed as the group mean and standard error of the mean. Three sets of data comparisons were performed via two tailed unpaired $t$-test: $N p c 1^{w t} v s N p c 1^{\text {ih }}$ mice fed a regular chow diet (\# $p \leq 0.05$; \#\# $p<0.01$; \#\# $p<0.001$; \#\#\# $p<0.0001$ ); Npc1 ${ }^{\text {nih }}$ mice receiving regular chow $v s ~ N p c 1^{\text {nih }}$ mice fed a two percent or six percent stanol-enriched chow $\operatorname{diet}\left({ }^{*} p \leq\right.$ $\left.0.05 ;^{* *} p<0.01{ }^{* * *} p<0.001 ;{ }^{* * *} p<0.0001\right)$. Data were statistically analyzed using GraphPad Prism software (version 6, GraphPad Software Inc, San Diego, CA, U.S; www.graphpad.com). 


\section{RESULTS}

Plant stanol supplementation delays body weight loss in $N p c 1^{\text {nih }}$ mice and reduces relative liver weight

As reduced weight gain reflects NPC1 disease progression in $N p c 1^{\text {nih }}$ mice, we monitored the body weight of $N p c 1^{\text {nih }}$ mice and assessed whether plant stanol supplementation influenced this parameter. From day 12 of the study, which coincided with the weaning period, untreated $N p c 1^{\text {nih }}$ mice were consistently smaller than $N p c 1^{w t}$ mice (Fig. $\left.1 \mathrm{~A}\right)$. $N p c 1^{\text {nih }}$ mice on a two percent plant stanol-enriched diet showed a modest increase in body weight compared to untreated $N p c 1^{\text {nih }}$ mice until day 22 of the study, although this did not reach statistical significance. On the other hand, six percent plant stanol supplementation effectively rescued $N p c 1^{\text {nih }}$ mice's weight between days 12 and 29 of the study. Furthermore, in the last week of the study, a trend towards increased body weight was observed in $N p c 1^{\text {nih }}$ mice on a six percent plant stanol-enriched diet compared to their untreated counterparts.

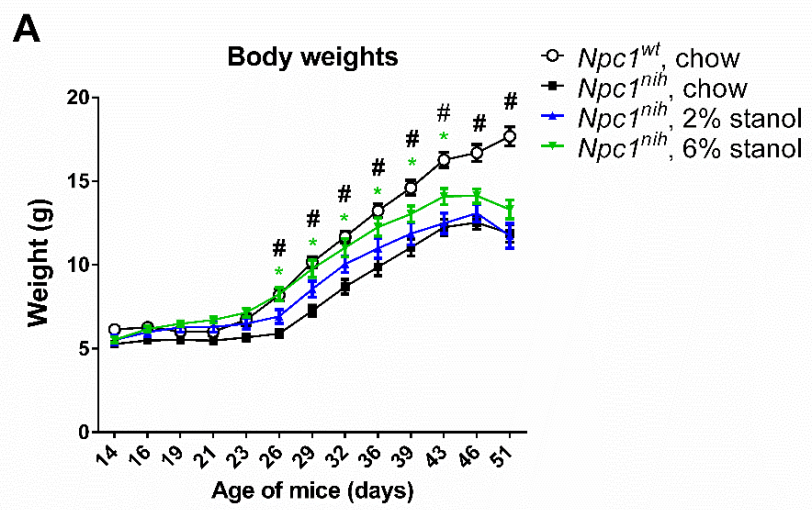

B

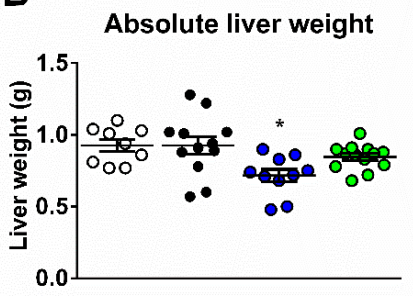

C

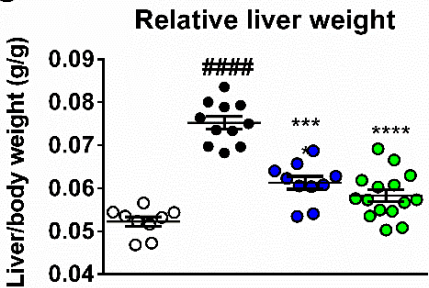

- Npc1wt, chow

- Npc1 $1^{\text {nih }}$ chow

- Npc1 $1^{n i h}, 2 \%$ stanol

- $N p c 1^{\text {nin }}, 6 \%$ stanol

Figure 1: Effect of dietary stanol supplementation on weight parameters. (A) Body weight of $N p c 1^{w t}$ and $N p c 1^{\text {nih }}$ mice throughout the study period. (B, C) Relative liver weight. Statistical analysis was performed by use of two-tailed unpaired $t$ test. $\mathrm{n}=10-16$ mice per group. $N p c 1^{\text {wt }} v s N p c 1^{\text {nih }}$ mice fed a regular chow diet $(\# p \leq 0.05 ; \# p<0.01$; \#\# $p<$ $0.001 ; \# \# \# p<0.0001) ; N p c 1^{\text {nih }}$ mice receiving regular chow $v s N p c 1^{\text {inh }}$ fed two percent or six percent stanol-enriched chow $\operatorname{diet}\left({ }^{*} p \leq 0.05 ;{ }^{* *} p<0.01 ;{ }^{* * *} p<0.001 ;{ }^{* * * *} p<0.0001\right)$. All error bars represent standard error of the mean. 
Additionally, liver weights were analyzed, as hepatomegaly is a prominent systemic feature of NPC1 disease. Although absolute liver weight was comparable between $N p c 1^{\text {nih }}$ mice and $N p c 1^{w t}$ mice, liver weights relative to body weight of untreated $N p c 1^{\text {nih }}$ mice were higher compared to $N p c 1^{w t}$ mice (Fig. 1B-C). Relevantly, relative liver weight of $N p c 1^{\text {nih }}$ mice fed a two percent and six percent plant stanol-enriched diet was reduced. In addition, absolute liver weights were reduced in $N p c 1^{\text {nih }}$ mice on a two percent plant stanol-supplemented diet. Overall, these results indicate that dietary supplementation with plant stanol esters ameliorates body weight gain and hepatomegaly in $N p c 1^{\text {nih }}$ mice.

\section{Decreased plasma and hepatic total cholesterol levels in $N p c 1^{\text {ih }}$ mice fed a plant stanol- enriched diet}

To assess the effect of dietary plant stanol supplementation on lipid metabolism of $N p c 1^{\text {nih }}$ mice, biochemical analyses of plasma and liver lipids were performed. In line with plant stanols' well-known plasma cholesterol lowering effect, $N p c 1^{\text {nih }}$ mice fed a plant stanolsupplemented diet displayed lower levels of plasma total cholesterol (Fig. 2A), but not of plasma total triglycerides (Fig. 2B) compared to untreated $N p c 1^{\text {nih }}$ mice. Of note, these effects were more pronounced following a six percent stanol enriched diet. Next, we analyzed hepatic lipid accumulation, a prominent systemic feature of NPC1 disease. As expected, untreated $N p c 1^{\text {nih }}$ mice displayed higher levels of hepatic cholesterol compared to $N p c 1^{w t}$ mice (Fig. 2C). Npc1 $1^{\text {nih }}$ mice that received plant stanol supplementation, particularly at six percent, showed prominently lower levels of hepatic total cholesterol than untreated $N p c 1^{\text {nih }}$ mice, indicating that plant stanol supplementation reduced hepatic cholesterol levels in a dosedependent manner (Fig. 2C). In contrast, plant stanol supplementation showed no effects on hepatic triglyceride accumulation of $N p c 1^{\text {nih }}$ mice, who displayed lower levels of liver triglycerides than $N p c 1^{w t}$ mice (Fig. 2D).

To better understand changes in hepatic cholesterol accumulation following plant stanol supplementation, hepatic gene expression analysis was performed on cluster of differentiation $36(\mathrm{Cd36})$ and scavenger receptor A $(S r-a)$, which mediate the uptake of modified lipoproteins in macrophages, such as those increased in NPC1 disease patients (37, 38); on Niemann-Pick type C2 ( $N p c 2)$, a protein that transfers free cholesterol within LELs to NPC1; on ATP-binding cassette sub-family G1 and G8 (Abcg1 and Abcg8), which mediate excess sterol efflux from leukocytes and hepatocytes, respectively (39); and on cytochrome P450 family 8 subfamily B member 1 (Cyp8b1), which promotes excess cholesterol excretion by mediating the synthesis of bile acids (Figs. 2E-J, respectively). Following two percent plant stanol administration, expression of $S r-a$ and $N p c 2$ decreased in the livers of $N p c 1^{\text {nih }}$ mice, suggesting a reduction in the uptake of pro-inflammatory modified lipoproteins by 
macrophages and lower build-up of free cholesterol in LELs of $N p c 1^{\text {nih }}$ mice. Furthermore, $N p c 1^{\text {nih }}$ mice on a two percent plant stanol-enriched diet displayed higher expression of Cyp8b1, suggesting increased conversion of excess hepatic cholesterol into bile acids. Likewise, $N p c 1^{\text {nih }}$ mice on a six percent plant stanol-enriched diet displayed lower hepatic expression of $S r-a$ and $N p c 2$ and increased expression of Cyp8b1 than their untreated counterparts. In addition to improving expression of the aforementioned genes, six percent plant stanol supplementation reduced hepatic expression of $C d 36$ and increased expression of $A b c g 8$, suggesting reduced uptake of modified lipoproteins and increased excretion of excess cholesterol in hepatocytes of $N p c 1^{\text {nih }}$ mice. Finally, $N p c 1^{\text {nih }}$ mice displayed lower hepatic expression of $A b c g 1$, suggesting reduced efflux of cholesterol and oxysterols in macrophages. Overall, these findings indicate that, besides lowering plasma cholesterol levels, plant stanols reduce cholesterol accumulation in the liver of $N p c 1^{\text {nih }}$ mice in a dose-dependent manner.
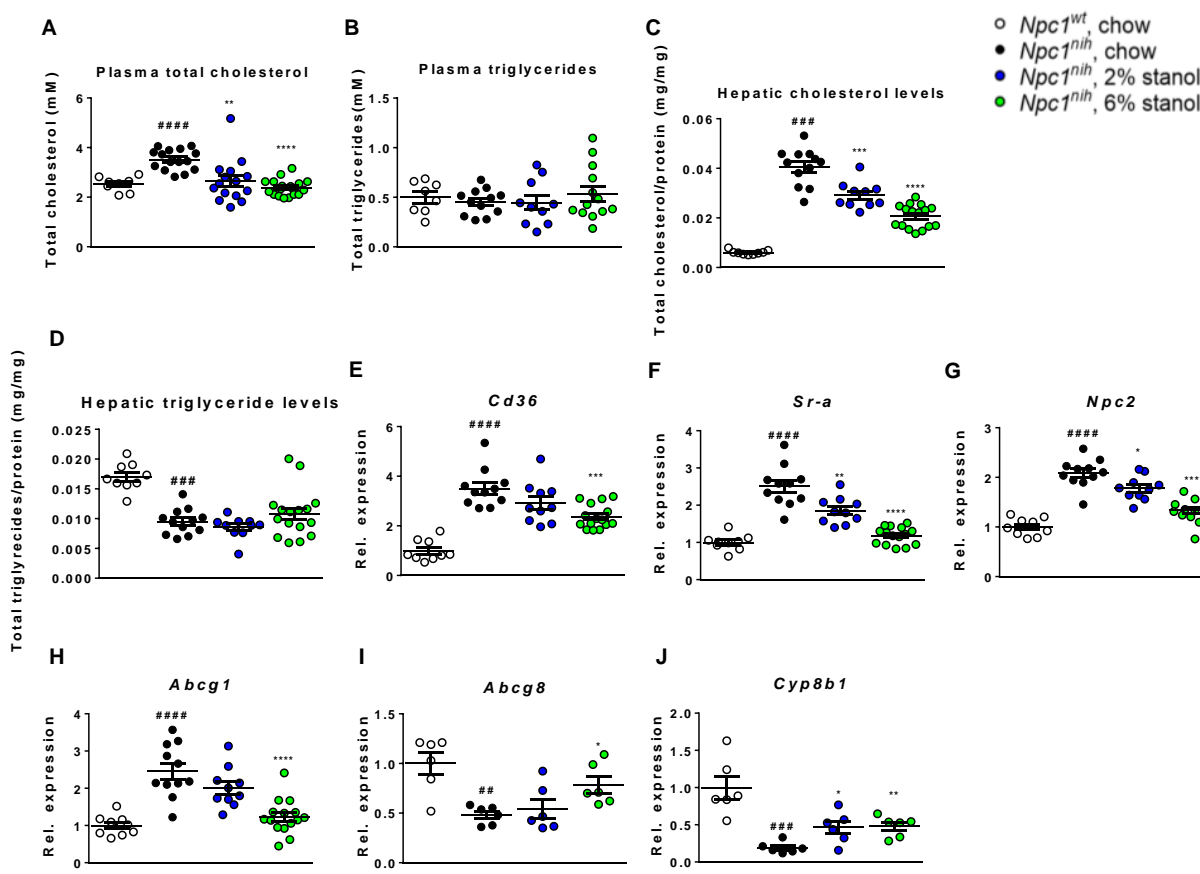

E

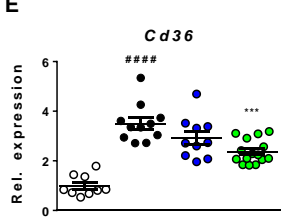

$\mathbf{F}$
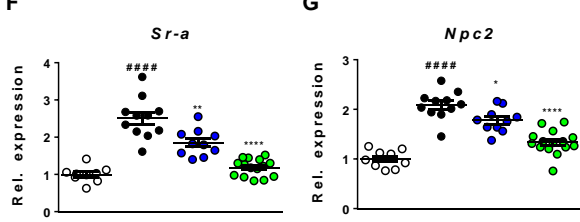

J

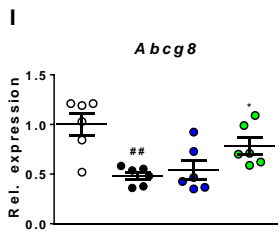

Figure 2: Lipid metabolism parameters. (A, B) Total plasma cholesterol and triglyceride levels of $N p c 1^{\text {wt }}$ and $N p c 1^{\text {nih }}$ mice on a regular and stanol-supplemented chow diet. (C, D) Liver cholesterol and triglyceride levels. (E-J) Hepatic lipid metabolism-related gene expression of $C d 36, S r-a, N p c 2, A b c g 1, A b c g 8, C y p 8 b 1$. Statistical analysis was performed by use of two-tailed unpaired $t$ test. $\mathrm{n}=9-15$ mice per group for liver gene expression analyses. $N p c 1^{w t} v s N p c 1^{\text {nih }}$ mice mice fed a regular chow diet (\#p $\leq 0.05 ; \# \#<0.01 ; \# \# p<0.001$; \#\#\# $p<0.0001)$; Npc1 $1^{\text {nih }}$ mice receiving regular chow vs $N p c 1^{\text {nih }}$ mice fed two percent or six percent stanol-enriched chow $\operatorname{diet}\left({ }^{*} p \leq 0.05 ;{ }^{* *} p<0.01\right.$; ${ }^{* * *} p<0.001$; $\left.^{* * *} p<0.0001\right)$. All error bars represent standard error of the mean. 
Dietary plant stanol supplementation improves hepatic damage and inflammation in Npc1 ${ }^{\text {nih }}$ mice

Following the observed improvements in hepatic cholesterol metabolism, we next investigated the effects of plant stanol supplementation on hepatic damage and inflammation. Plasma alanine transaminase (ALT) levels of untreated $N p c 1^{\text {nih }}$ mice showed a near four-fold increase in relation to $N p c 1^{w t}$ mice, indicating increased liver damage in $N p c 1^{\text {nih }}$ mice (Fig. 3A). Remarkably, after dietary plant stanol supplementation, plasma ALT levels of $N p c 1^{\text {nih }}$ mice were comparable to $N p c 1^{w t}$ mice, indicating a strong decrease in overall liver damage. We further looked into hepatic inflammatory status via immunohistochemistry by targeting measuring CD68 and Mac-1 proteins, which identify resident hepatic macrophages and infiltrated neutrophils and macrophages, respectively (Figs. 3B-E). Untreated Npc1 ${ }^{\text {nih }}$ mice displayed higher levels of immune cells in both immunostainings, indicating prominent hepatic inflammation in $N p c 1^{\text {nih }}$ mice. The number of hepatic immune cells, particularly in the case of infiltrated neutrophils and macrophages, was reduced following plant stanol supplementation. To further assess the effects of plant stanol supplementation on liver inflammation, hepatic gene expression analyses were performed on tumor necrosis factor alpha $(\operatorname{Tnf}-\alpha)$, cluster of differentiation 68 (Cd68), cathepsin D (Ctsd), macrophage inflammatory protein 2 (Mip2), chemokine (C-C motif) ligand 3 (Ccl3) and arginase 1 (Arg1) (Figs. 3F-K). Hepatic gene expression of inflammatory markers was consistently increased in untreated $N p c 1^{\text {ih }}$ mice compared to $N p c 1^{w t}$ mice, and decreased in the case of Arg1, a marker for alternatively activated macrophages. Both two and six percent plant stanol supplementation reversed these observations, supporting the aforementioned findings that $N p c 1^{\text {nih }}$ mice fed a plant stanol esters-supplemented diet display lower hepatic inflammation and damage. 


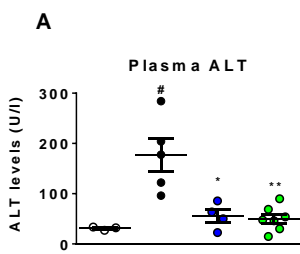

c

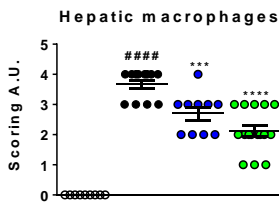

B Npc1wt, chow

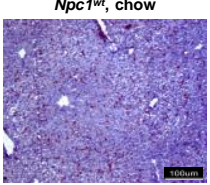

$N p 1^{\text {nih }}, 2 \%$ stanols
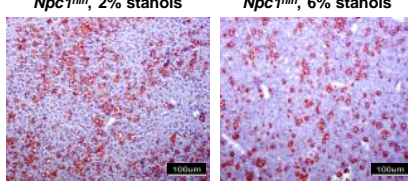

D

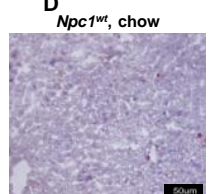

$N p 1^{\text {nih }}, 2 \%$ stanols

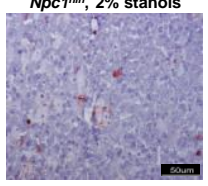

J

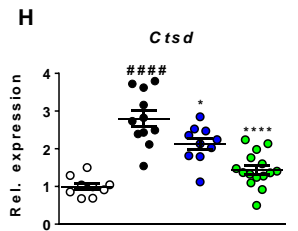

I

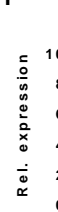

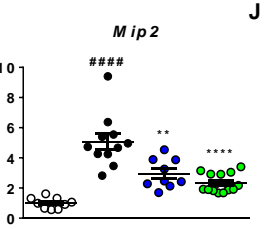

E

Infiltrated $m$ acrophages and neutrophils

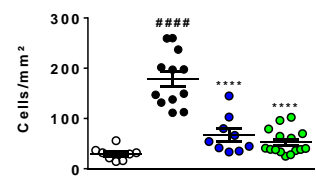

- Npc1wt, chow

- $N p c 1^{\text {nih }}$, chow

- Npc1 ${ }^{\text {ninh' }}, 2 \%$ stanol

- $N p c 1^{\text {nith }}, 6 \%$ stanol
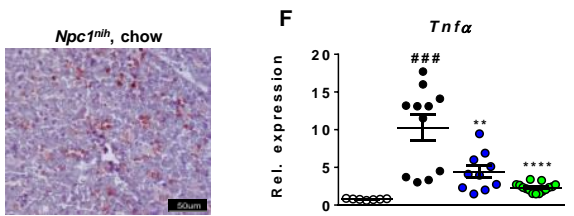

$N p c 1^{\text {nih }}, 6 \%$ stanols

G

Figure 3: Effect of dietary stanol supplementation on hepatic inflammation. (A) Plasma ALT levels. (B-E) Representative pictures of liver sections stained for hepatic macrophages (CD68) and infiltrated macrophages and neutrophils (Mac-1). CD68 immunostainings were scored (B,C), whereas Mac-1 positive cells were counted (D,E). (F-K) Hepatic gene expression of inflammatory markers Tnf- $\alpha, C d 68, C t d s$, Mip2, Ccl3 and Arg1. Statistical analysis was performed by use of two-tailed unpaired $t$ test. $\mathrm{n}=9-15$ mice per group for liver gene expression analyses. $N p c 1^{w t} v s$ $N p c 1^{\text {nih }}$ mice fed a regular chow $\operatorname{diet}(\# p \leq 0.05 ; \# \#<0.01$; \#\# $p<0.001$; \#\#\# $p<0.0001)$; Npc1 ${ }^{\text {nih }}$ mice receiving regular chow $v s N p c 1^{\text {nih }}$ mice fed two percent or six percent stanol-enriched chow $\operatorname{diet}\left({ }^{*} p \leq 0.05 ;{ }^{* *} p<0.01{ }^{* * * *} p<0.001{ }^{* * * *} p<\right.$ 0.0001). All error bars represent standard error of the mean.

Plant stanol supplementation shifts plasma profile of immune cells towards an antiinflammatory phenotype

To better understand the effects of plant stanol supplementation on systemic inflammation, we investigated monocyte and T-cell populations by FACS analysis in the blood of 35 and 49 days old $N p c 1^{\text {nih }}$ mice. To analyse the profile of circulating monocytes, we targeted Ly6C, a protein highly expressed in pro-inflammatory monocytes $(30,40,41)$. Untreated $N p c 1^{\text {nih }}$ mice displayed higher relative levels of Ly6 $\mathrm{C}^{\text {high }}$ monocytes and lower relative levels of Ly6Clow monocytes in the blood than $N p c 1^{w t}$ mice on both time points (Fig. 4A-B), suggesting higher amounts of pro-inflammatory monocytes and lower levels of anti-inflammatory monocytes, respectively. While $N p c 1^{\text {nih }}$ mice on a two percent plant stanol diet displayed lower levels of 
Ly6Chigh monocytes at 49 days old alone, six percent plant stanol supplementation reduced the relative amount of circulating Ly6Chigh monocytes on both timepoints, suggesting a reduction in circulating pro-inflammatory monocytes. Furthermore, for two and six percent plant stanol supplementation, a trend towards an increase in blood Ly6Clow monocytes was observed in 49 days old $N p c 1^{\text {nih }}$ mice. In addition, six percent plant stanol supplementation effectively triggered an increase in circulating Ly6C $\mathrm{C}^{\text {low }}$ monocytes in 35 days old $N p c 1^{\text {nih }}$ mice. Concerning blood T-cell populations, untreated $N p c 1^{\text {nih }}$ mice displayed higher levels of CD8 ${ }^{+}$ T-cells compared to $N p c 1^{w t}$ mice at 35 days of age, but not at 49 days old, whereas levels of $\mathrm{CD}^{+}$T-cells were lower in untreated Npc1 ${ }^{\text {nih }}$ mice at both time points (Fig. 4C-D). Although

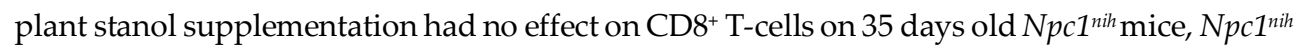
mice following six percent plant stanol supplementation displayed lower levels of $\mathrm{CD}^{+} \mathrm{T}$ cells at 49 days of age. Finally, although plant stanol supplementation did not significantly increase circulating helper T-cells in $N p c 1^{\text {nih }}$ mice, a trend was observed suggesting this effect for six percent plant stanol supplementation in 35 days old $N p c 1^{\text {nih }}$ mice. Overall, these results indicate that dietary plant stanol supplementation shifted the ratio of pro- and antiinflammatory circulating monocytes and T-cells towards a more anti-inflammatory phenotype.

A

Relative blood pro-inflam matory monocytes

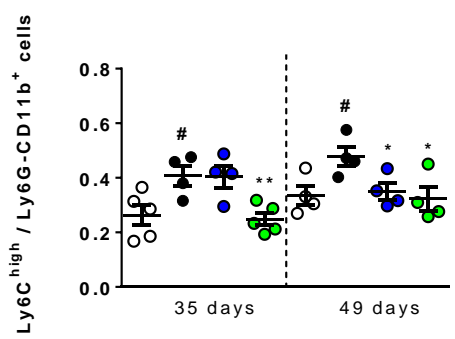

C

Relative blood cytotoxic T-cells

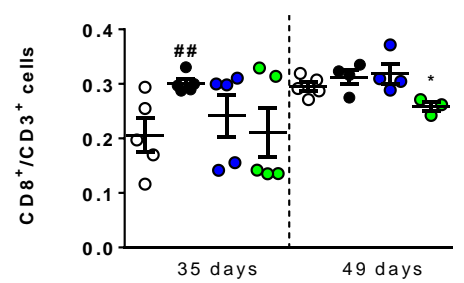

B

Relative blood anti-inflam matory monocytes

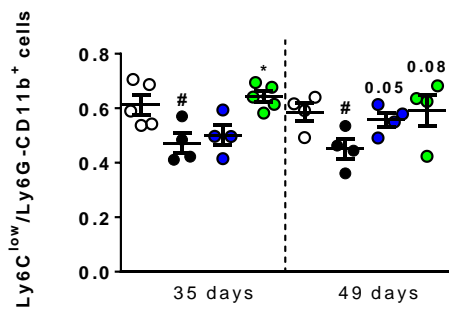

D Relative blood helper T-cells

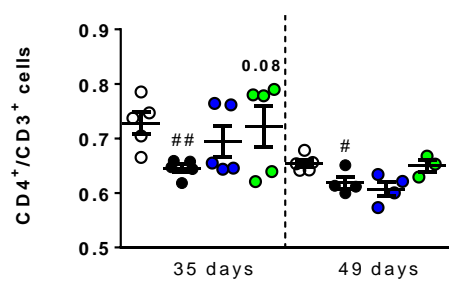

Figure 4: Effect of dietary stanol supplementation on plasma monocytes and T-cells phenotype. (A-H) Relative levels of plasma pro-inflammatory (LyC6 $\left.6^{\text {high }}\right)$ and anti-inflammatory (LyC6 $\left.6^{\text {low }}\right)$ monocytes, as well as cytotoxic $(\mathrm{CD} 8+)$ and 
helper T-cells (CD4+) were measured by FACS analysis on week 3 and 5 of the study, when mice were 35 and 49 days old. Statistical analysis was performed by use of two-tailed unpaired $t$ test. $\mathrm{n}=5$ mice per group. $N p c 1^{w t} v s N p c 1^{\text {nih }}$ mice fed a regular chow $\operatorname{diet}(\# p \leq 0.05 ; \# p<0.01$; \#\# $p<0.001$; \#\#\# $p<0.0001) ; N p c 1^{\text {nih }}$ mice receiving regular chow $v s N p c 1^{\text {nih }}$ mice fed two percent or six percent stanol-enriched chow $\operatorname{diet}\left({ }^{*} p \leq 0.05 ;{ }^{* *} p<0.01\right.$; $^{* * *} p<0.001$; $\left.^{* * *} p<0.0001\right)$. All error bars represent standard error of the mean.

Altogether, these findings indicate that dietary plant stanol esters supplementation improves hepatic lipid metabolism and reduces damage and inflammation in NPC1 disease. In addition, plant stanol supplementation shifts the phenotype of blood immune cells towards a more anti-inflammatory profile in NPC1 disease, particularly at higher concentrations 


\section{DISCUSSION}

In NPC1 disease, whole-body lysosomal lipid accumulation triggers a cascade of pathological events that culminates in a wide range of peripheral and neurological symptoms. In addition, early diagnosis and effective therapeutic tools are currently lacking for NPC1 disease, making it a severe and lethal condition that warrants further research in order to improve quality of life and lifespan of patients. In this study, we show that dietary plant stanol esters supplementation improves progressive weight loss, as well as hepatic cholesterol accumulation and damage in a murine model for NPC1 disease. In addition, the current study shows that dietary plant stanol supplementation shifts the profile of blood immune cells towards a more anti-inflammatory phenotype. Based on these findings, we propose that dietary plant stanol supplementation should be further investigated as a complementary tool to ameliorate hepatic symptoms and the phenotype of blood immune cells in NPC1 disease patients.

While the mechanisms underlying the beneficial effects of stanols are yet to be fully elucidated, cumulative evidence indicates that these molecules interfere with cholesterol micellar solubilization in the intestines and may further inhibit cholesterol absorption and stimulate cholesterol excretion by activation of liver $X$ receptor (LXR) transcription factor (42). As such, clinical benefits of increased plant stanol ester consumption are largely attributed to reduced dietary cholesterol absorption and consequent lowering of plasma cholesterol levels. In this study, increased plant stanol ester consumption induced a reduction in plasma and liver cholesterol levels in Npc1 $1^{\text {iih }}$ mice, in line with results from a previous NASH study (43). In addition to the effects of plant stanols on cholesterol absorption, a growing body of findings indicates that these molecules have anti-inflammatory and immunomodulatory properties (44). In a previous ex vivo study, sitostanol administration to mouse bone marrow-derived macrophages was shown to induce an anti-inflammatory effect independent of LXR activation. It should be noted that, since diets were not supplemented with cholesterol, mice in this study consumed low amounts of cholesterol. As such, although we cannot exclude a beneficial effect from reduced intestinal cholesterol absorption in $N p c 1^{\text {nih }}$ mice following increased plant stanol consumption, it is likely that plant stanols' anti-inflammatory properties also contributed to the observed improvement in hepatic inflammation and damage. Furthermore, in a previous study, pharmacological LXR activation increased brain cholesterol excretion and ameliorated disease burden in $\mathrm{Npc1}^{-/}$mice (45). As previously mentioned, plant stanol molecules are known LXR activators. As such, it is likely that increased plant stanol molecules improved hepatic pathology in $N p c 1^{\text {nih }}$ mice via a variety of mechanisms, namely reduced intestinal cholesterol absorption, anti-inflammatory effects and LXR activation. In addition to a local effect on hepatic inflammation, dietary plant stanol ester 


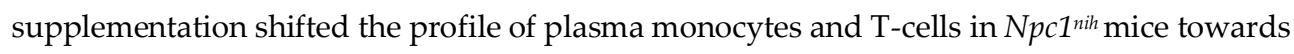
a more anti-inflammatory phenotype, particularly in the former population. Previously, Brüll et al. have demonstrated that sitostanol administration elicits a TLR2-dependent T-helper 1 shift in human peripheral blood mononuclear cells cultures, even at very low concentrations. Further studies on asthma patients-derived peripheral blood mononuclear cells cultures confirmed the findings that sitostanol administration induces a T-helper 1 cell response and, in addition, lead to an increase in numbers and activity of regulatory T cells (46). It is thus possible that the shift in phenotype of circulating immune cells of $N p c 1^{\text {nih }}$ mice following plant stanol ester supplementation is derived from the direct effect of plant stanols on circulating immune cell populations. Of note, previous studies found that phytosterol supplementation ameliorates inflammation and oxidative stress in Crohn's disease, a disorder which occurs in several NPC1 disease patients $(47,48)$. Considering the anti-inflammatory effects attributed to plant stanols, it is possible that a plant stanol-enriched diet could also ameliorate the intestinal problems of NPC1 disease patients. If so, this would further enhance the application of plant stanol supplementation as an additional therapeutical tool in NPC1 disease. Of note, unlike stanols, phytosterol molecules are prone to oxidation $(49,50)$, and may therefore have pro-inflammatory effects if consumed in high amounts. Given the importance of inflammation in NPC1 disease burden $(30,51,52)$, phytosterol supplementation should thus be regarded with caution.

Currently, Miglustat is the only approved drug for the treatment of NPC1 disease symptoms. While clinical observations indicate that Miglustat delays progression of neurological deterioration, the effects of Miglustat on systemic features of NPC1 disease remain largely unexplored, as it is specifically prescribed for amelioration of neurological symptoms $(10,11)$. On the other hand, 2-hydroxypropyl- $\beta$-cyclodextrin, which previously has been shown to improve systemic symptoms of NPC1 disease in murine models, is currently being evaluated in clinical trials regarding its efficacy on neurological and systemic symptoms $(12,53)$. While neuroinflammation and degeneration are the largest contributors to reduced quality of life and lifespan of NPC1 disease patients, hepatic, splenic, intestinal and lung dysfunction are also observed in a significant amount of NPC1 disease patients, particularly in early onset NPC1 disease cases $(6,7,47,48)$. As such, further strategies to reduce systemic manifestations of NPC1 disease and to complement neurological-targeted treatments are required. In the past, cholesterol-lowering therapeutic strategies such as dietary cholesterol restriction and statin administration have been explored in murine NPC1 disease models and patients and found to ameliorate hepatic symptoms (54-56). Of note, combined use of dietary plant stanol supplementation and statins amplifies the cholesterol-lowering properties of each intervention in hypercholesterolemic patients $(57,58)$. As such, it is possible that the administration of statins and plant stanol esters simultaneously has additional benefits to 
systemic manifestations of NPC1 disease and to improving life quality of NPC1 disease patients. On the other hand, combining several methods to reduce peripheral cholesterol has been controversial in the clinical setting, demanding the need for additional clinical trials to assess the clinical use of combinational approaches to reduce plasma cholesterol (59).

Overall, considering the promising results here described, we propose that dietary plant stanol esters supplementation should be further investigated as a complementary therapeutical tool to ameliorate hepatic symptoms and the phenotype of blood immune cells in NPC1 disease. Plant stanol esters are widely available in functional foods, such as margarine spreads, and have been studied in human populations where it showed very minor side-effects, even when consumed in higher concentrations $(24,60,61)$. Nonetheless, it should be further investigated whether increased stanol consumption bears so far unknown sideeffects in NPC1 disease, as well as plant stanols effects on the nervous system. 


\section{REFERENCES}

1. Geberhiwot T, Moro A, Dardis A, Ramaswami U, Sirrs S, Marfa MP, et al. Consensus clinical management guidelines for Niemann-Pick disease type C. Orphanet Journal of Rare Diseases. 2018;13(1):50.

2. Li X, Saha P, Li J, Blobel G, Pfeffer SR. Clues to the mechanism of cholesterol transfer from the structure of NPC1 middle lumenal domain bound to NPC2. Proc Natl Acad Sci U S A. 2016;113(36):10079-84.

3. Höglinger D, Burgoyne T, Sanchez-Heras E, Hartwig P, Colaco A, Newton J, et al. NPC1 regulates ER contacts with endocytic organelles to mediate cholesterol egress. Nature Communications. 2019;10(1):4276.

4. Pfeffer SR. Clues to NPC1-mediated cholesterol export from lysosomes. Proc Natl Acad Sci U S A. 2016;113(29):7941-3.

5. Millat G, Marçais C, Tomasetto C, Chikh K, Fensom AH, Harzer K, et al. NiemannPick C1 Disease: Correlations between NPC1 Mutations, Levels of NPC1 Protein, and Phenotypes Emphasize the Functional Significance of the Putative Sterol-Sensing Domain and of the Cysteine-Rich Luminal Loop. American Journal of Human Genetics. 2001;68(6):1373-85.

6. Patterson MC, Clayton P, Gissen P, Anheim M, Bauer P, Bonnot O, et al. Recommendations for the detection and diagnosis of Niemann-Pick disease type C. Neurology: Clinical Practice. 2017;7(6):499.

7. Kelly DA, Portmann B, Mowat AP, Sherlock S, Lake BD. Niemann-Pick disease type $\mathrm{C}$ : diagnosis and outcome in children, with particular reference to liver disease. J Pediatr. 1993;123(2):242-7.

8. Staretz-Chacham O, Aviram M, Morag I, Goldbart A, Hershkovitz E. Pulmonary involvement in Niemann-Pick C type 1. European Journal of Pediatrics. 2018;177(11):1609-15. 9. Birch NC, Radio S, Horslen S. Metastatic Hepatocellular Carcinoma in a Patient with Niemann-Pick Disease, Type C. Journal of Pediatric Gastroenterology and Nutrition. 2003;37(5).

10. Pineda M, Walterfang M, Patterson MC. Miglustat in Niemann-Pick disease type C patients: a review. Orphanet journal of rare diseases. 2018;13(1):140-.

11. Pineda M, Perez-Poyato MS, O'Callaghan M, Vilaseca MA, Pocovi M, Domingo R, et al. Clinical experience with miglustat therapy in pediatric patients with Niemann-Pick disease type C: A case series. Molecular Genetics and Metabolism. 2010;99(4):358-66.

12. Ory DS, Ottinger EA, Farhat NY, King KA, Jiang X, Weissfeld L, et al. Intrathecal 2hydroxypropyl-\&\#x3b2;-cyclodextrin decreases neurological disease progression in Niemann-Pick disease, type C1: a non-randomised, open-label, phase 1\&\#x2013;2 trial. The Lancet. 2017;390(10104):1758-68. 
13. Fog CK, Kirkegaard T. Animal models for Niemann-Pick type C: implications for drug discovery \& development. Expert Opinion on Drug Discovery. 2019;14(5):499-509.

14. Fu R, Yanjanin NM, Bianconi S, Pavan WJ, Porter FD. Oxidative stress in NiemannPick disease, type C. Mol Genet Metab. 2010;101(2-3):214-8.

15. Rimkunas VM, Graham MJ, Crooke RM, Liscum L. TNF-\{alpha\} plays a role in hepatocyte apoptosis in Niemann-Pick type C liver disease. J Lipid Res. 2009;50(2):327-33.

16. Kulinski A, Vance JE. Lipid Homeostasis and Lipoprotein Secretion in NiemannPick C1-deficient Hepatocytes. Journal of Biological Chemistry. 2007;282(3):1627-37.

17. Lopez ME, Klein AD, Hong J, Dimbil UJ, Scott MP. Neuronal and epithelial cell rescue resolves chronic systemic inflammation in the lipid storage disorder Niemann-Pick C. Human Molecular Genetics. 2012;21(13):2946-60.

18. Hendrikx T, Walenbergh SM, Hofker MH, Shiri-Sverdlov R. Lysosomal cholesterol accumulation: driver on the road to inflammation during atherosclerosis and non-alcoholic steatohepatitis. Obes Rev. 2014;15(5):424-33.

19. Houben T, Oligschlaeger Y, Bitorina AV, Hendrikx T, Walenbergh SMA, Lenders M$\mathrm{H}$, et al. Blood-derived macrophages prone to accumulate lysosomal lipids trigger oxLDLdependent murine hepatic inflammation. Scientific Reports. 2017;7(1):12550.

20. Jeurissen MLJ, Walenbergh SMA, Houben T, Gijbels MJJ, Li J, Hendrikx T, et al. Prevention of oxLDL uptake leads to decreased atherosclerosis in hematopoietic NPC1deficient Ldlr-/- mice. Atherosclerosis. 2016;255:59-65.

21. Tabas I, Garcia-Cardena G, Owens GK. Recent insights into the cellular biology of atherosclerosis. J Cell Biol. 2015;209(1):13-22.

22. Libby P. Inflammation in Atherosclerosis. Arteriosclerosis, Thrombosis, and Vascular Biology. 2012;32(9):2045.

23. Plat J, Hendrikx T, Bieghs V, Jeurissen MLJ, Walenbergh SMA, van Gorp PJ, et al. Protective Role of Plant Sterol and Stanol Esters in Liver Inflammation: Insights from Mice and Humans. PLOS ONE. 2014;9(10):e110758.

24. Gylling H, Plat J, Turley S, Ginsberg HN, Ellegård L, Jessup W, et al. Plant sterols and plant stanols in the management of dyslipidaemia and prevention of cardiovascular disease. Atherosclerosis. 2014;232(2):346-60.

25. Gylling H, Simonen P. Phytosterols, Phytostanols, and Lipoprotein Metabolism. Nutrients. 2015;7(9):7965-77.

26. Ostlund RE, McGill JB, Zeng C-M, Covey DF, Stearns J, Stenson WF, et al. Gastrointestinal absorption and plasma kinetics of soy $\Delta 5$-phytosterols and phytostanols in humans. American Journal of Physiology-Endocrinology and Metabolism. 2002;282(4):E911E6. 
27. Katan MB, Grundy SM, Jones P, Law M, Miettinen T, Paoletti R. Efficacy and Safety of Plant Stanols and Sterols in the Management of Blood Cholesterol Levels. Mayo Clinic Proceedings. 2003;78(8):965-78.

28. Nguyen TT. The Cholesterol-Lowering Action of Plant Stanol Esters. The Journal of Nutrition. 1999;129(12):2109-12.

29. Thompson GR, Grundy SM. History and Development of Plant Sterol and Stanol Esters for Cholesterol-Lowering Purposes. The American Journal of Cardiology. 2005;96(1, Supplement):3-9.

30. Smith D, Wallom K-L, Williams IM, Jeyakumar M, Platt FM. Beneficial effects of antiinflammatory therapy in a mouse model of Niemann-Pick disease type C1. Neurobiology of Disease. 2009;36(2):242-51.

31. Gemma V, Soumaya B-A, Elisa D, Lina B, Teresa P. Phytosterols and Inflammation. Current Medicinal Chemistry. 2018;25:1-9.

32. Parra J, Klein AD, Castro J, Morales MG, Mosqueira M, Valencia I, et al. Npc1 deficiency in the C57BL/6J genetic background enhances Niemann-Pick disease type C spleen pathology. Biochemical and Biophysical Research Communications. 2011;413(3):400-6.

33. Bieghs V, Wouters K, van Gorp PJ, Gijbels MJ, de Winther MP, Binder CJ, et al. Role of scavenger receptor $\mathrm{A}$ and CD36 in diet-induced nonalcoholic steatohepatitis in hyperlipidemic mice. Gastroenterology. 2010;138(7):2477-86, 86 e1-3.

34. Bieghs V, Verheyen F, van Gorp PJ, Hendrikx T, Wouters K, Lutjohann D, et al. Internalization of modified lipids by CD36 and SR-A leads to hepatic inflammation and lysosomal cholesterol storage in Kupffer cells. PLoS One. 2012;7(3):e34378.

35. Bieghs V, van Gorp PJ, Walenbergh SM, Gijbels MJ, Verheyen F, Buurman WA, et al. Specific immunization strategies against oxidized low-density lipoprotein: a novel way to reduce nonalcoholic steatohepatitis in mice. Hepatology. 2012;56(3):894-903.

36. Thelen KM, Laaksonen R, Päivä H, Lehtimäki T, Lütjohann D. High-Dose Statin Treatment Does Not Alter Plasma Marker for Brain Cholesterol Metabolism in Patients With Moderately Elevated Plasma Cholesterol Levels. The Journal of Clinical Pharmacology. 2006;46(7):812-6.

37. Kelley JL, Ozment TR, Li C, Schweitzer JB, Williams DL. Scavenger receptor-A (CD204): a two-edged sword in health and disease. Crit Rev Immunol. 2014;34(3):241-61.

38. Porter FD, Scherrer DE, Lanier MH, Langmade SJ, Molugu V, Gale SE, et al. Cholesterol oxidation products are sensitive and specific blood-based biomarkers for Niemann-Pick C1 disease. Sci Transl Med. 2010;2(56):56ra81-56ra81.

39. Tarr PT, Tarling EJ, Bojanic DD, Edwards PA, Baldán Á. Emerging new paradigms for ABCG transporters. Biochimica et Biophysica Acta (BBA) - Molecular and Cell Biology of Lipids. 2009;1791(7):584-93. 
40. Kimball A, Schaller M, Joshi A, Davis FM, denDekker A, Boniakowski A, et al. Ly6C(Hi) Blood Monocyte/Macrophage Drive Chronic Inflammation and Impair Wound Healing in Diabetes Mellitus. Arterioscler Thromb Vasc Biol. 2018;38(5):1102-14.

41. Yang J, Zhang L, Yu C, Yang X-F, Wang H. Monocyte and macrophage differentiation: circulation inflammatory monocyte as biomarker for inflammatory diseases. Biomark Res. 2014;2(1):1-.

42. Smet ED, Mensink RP, Plat J. Effects of plant sterols and stanols on intestinal cholesterol metabolism: Suggested mechanisms from past to present. Molecular Nutrition \& Food Research. 2012;56(7):1058-72.

43. Plat J, Hendrikx T, Bieghs V, Jeurissen MLJ, Walenbergh SMA, van Gorp PJ, et al. Protective role of plant sterol and stanol esters in liver inflammation: insights from mice and humans. PloS one. 2014;9(10):e110758-e.

44. Plat J, Baumgartner S, Vanmierlo T, Lütjohann D, Calkins KL, Burrin DG, et al. Plantbased sterols and stanols in health \& disease: "Consequences of human development in a plant-based environment?". Progress in Lipid Research. 2019;74:87-102.

45. Repa JJ, Li H, Frank-Cannon TC, Valasek MA, Turley SD, Tansey MG, et al. Liver X Receptor Activation Enhances Cholesterol Loss from the Brain, Decreases Neuroinflammation, and Increases Survival of the NPC1 Mouse. The Journal of Neuroscience. 2007;27(52):14470.

46. Brüll F, Mensink RP, Steinbusch MF, Husche C, Lütjohann D, Wesseling G-J, et al. Beneficial Effects of Sitostanol on the Attenuated Immune Function in Asthma Patients: Results of an In Vitro Approach. PLOS ONE. 2012;7(10):e46895.

47. Schwerd T, Pandey S, Yang H-T, Bagola K, Jameson E, Jung J, et al. Impaired antibacterial autophagy links granulomatous intestinal inflammation in Niemann-Pick disease type C1 and XIAP deficiency with NOD2 variants in Crohn\&\#039;s disease. Gut. 2017;66(6):1060.

48. Cavounidis A, Uhlig HH. Crohn's Disease in Niemann-Pick Disease Type C1: Caught in the Cross-Fire of Host-Microbial Interactions. Digestive Diseases and Sciences. 2018;63(4):811-3.

49. Vanmierlo T, Husche C, Schött HF, Pettersson H, Lütjohann D. Plant sterol oxidation products - Analogs to cholesterol oxidation products from plant origin? Biochimie. 2013;95(3):464-72.

50. Plat J, Brzezinka H, Lütjohann D, Mensink RP, von Bergmann K. Oxidized plant sterols in human serum and lipid infusions as measured by combined gas-liquid chromatography-mass spectrometry. Journal of Lipid Research. 2001;42(12):2030-8.

51. Houben T, Magro dos Reis I, Oligschlaeger Y, Steinbusch H, Gijbels MJJ, Hendrikx $\mathrm{T}$, et al. Pneumococcal Immunization Reduces Neurological and Hepatic Symptoms in a Mouse Model for Niemann-Pick Type C1 Disease. Frontiers in Immunology. 2019;9:3089. 
52. Platt N, Speak AO, Colaco A, Gray J, Smith DA, Williams IM, et al. Immune dysfunction in Niemann-Pick disease type C. Journal of Neurochemistry. 2016;136(S1):74-80. 53. Ramirez CM, Liu B, Taylor AM, Repa JJ, Burns DK, Weinberg AG, et al. Weekly cyclodextrin administration normalizes cholesterol metabolism in nearly every organ of the Niemann-Pick type C1 mouse and markedly prolongs life. Pediatric research. 2010;68(4):30915.

54. Somers KL, Brown DE, Fulton R, Schultheiss PC, Hamar D, Smith MO, et al. Effects of dietary cholesterol restriction in a feline model of Niemann-Pick type C disease. Journal of Inherited Metabolic Disease. 2001;24(4):427-36.

55. Erickson RP, Garver WS, Camargo F, Hossian GS, Heidenreich RA. Pharmacological and genetic modifications of somatic cholesterol do not substantially alter the course of CNS disease in Niemann-Pick C mice. Journal of Inherited Metabolic Disease. 2000;23(1):54-62.

56. Patterson MC, Di Bisceglie AM, Higgins JJ, Abel RB, Schiffmann R, Parker CC, et al. The effect of cholesterol-lowering agents on hepatic and plasma cholesterol in Niemann-Pick disease type C. Neurology. 1993;43(1 Part 1):61.

57. Rosin S, Ojansivu I, Kopu A, Keto-Tokoi M, Gylling H. Optimal Use of Plant Stanol Ester in the Management of Hypercholesterolemia. Cholesterol. 2015;2015:706970-.

58. Scholle JM, Baker WL, Talati R, Coleman CI. The Effect of Adding Plant Sterols or Stanols to Statin Therapy in Hypercholesterolemic Patients: Systematic Review and MetaAnalysis. Journal of the American College of Nutrition. 2009;28(5):517-24.

59. Linnebur SA, Capell WH, Saseen JJ, Wolfe P, Eckel RH. Plant sterols added to combination statin and colesevelam hydrochloride therapy failed to lower low-density lipoprotein cholesterol concentrations. Journal of Clinical Lipidology. 2007;1(6):626-33.

60. Berger A, Jones PJH, Abumweis SS. Plant sterols: factors affecting their efficacy and safety as functional food ingredients. Lipids in Health and Disease. 2004;3(1):5.

61. Gylling H, Hallikainen M, Nissinen MJ, Miettinen TA. The effect of a very high daily plant stanol ester intake on serum lipids, carotenoids, and fat-soluble vitamins. Clinical Nutrition. 2010;29(1):112-8. 



\section{Chapter 3}

Anti-Inflammatory Effects of Dietary Plant Stanol Supplementation Are Largely Dependent on the Intake of Cholesterol in a Mouse Model of Metabolic Inflammation.

Inês Magro dos Reis, Tom Houben, Marion J. J. Gijbels, Dieter Lütjohann, Jogchum Plat, Ronit Shiri-Sverdlov

Biomedicines, 2021; 9(5), 518 


\section{ABSTRACT}

The prevalence of metabolic disorders characterized by chronic inflammation has been on a sharp rise for decades. As such, tools that address metabolic and inflammatory dysregulation are of great importance. Plant stanols are well-known for reducing intestinal cholesterol absorption and may also have direct anti-inflammatory effects. In this study, our aim was to investigate to what extent the benefits of dietary plant stanol supplementation depend on dietary cholesterol intake in an experimental mouse model for cholesterol-induced metabolic inflammation. Here, we used $\mathrm{Ldlr}^{-/-}$mice transplanted with $\mathrm{Np} c 1^{\text {nih }}$-derived bone marrow, featuring feature bone marrow-derived immune cells characterized by chronic inflammation induced by lysosomal lipid accumulation. $N p c 1^{\text {nih }}$ - and $N p c 1^{\text {wt }}$-transplanted mice were placed on either a high fat, high cholesterol (HFC) or on a chow diet low in cholesterol, with or without $2 \%$ plant stanols supplementation. At the end of the study, the metabolic and inflammatory status of the mice was analyzed. Plant stanol supplementation to the HFC diet reduced liver cholesterol levels and improved lipid metabolism and liver inflammation, particularly in Npc1 ${ }^{\text {nih }}$-tp mice. In contrast, plant stanol supplementation to the chow diet did not significantly improve the aforementioned parameters, though similar reductive trends to those in the HFC diet setting were observed regarding liver cholesterol accumulation and liver inflammatory markers. The effects of dietary plant stanol supplementation on dietary cholesterol-induced inflammation are largely dependent on dietary cholesterol intake. Future research should verify whether other models of metabolic inflammation exhibit similar stanol-related effects on inflammation. 


\section{INTRODUCTION}

Despite efforts to raise awareness on the importance of a healthy lifestyle, the prevalence of obesity accompanied by metabolic syndrome has been on a sharp rise for decades (1). Individuals who meet at least three of the following criteria are diagnosed with metabolic syndrome: increased hip-to-waist ratio, hypertriglyceridemia, hyperglycemia, low highdensity lipoprotein plasma levels, and hypertension. Individuals who suffer from metabolic syndrome are at high risk of developing severe non-communicable diseases such as type II diabetes mellitus, atherosclerosis, liver and cardiovascular diseases, cancer, and even dementia and depression. Not only are the aforementioned diseases associated with reduced quality of life, they also account for nearly $70 \%$ of all premature deaths worldwide (2). Overall, it is clear that a concerted effort is required to increase the awareness of the strengths of lifestyle-related prevention strategies as well as to develop efficient strategies to target metabolic syndrome and its associated disease burden.

Plant stanols are plant-derived molecules that result from the saturation of plant sterols, which are analogous to cholesterol in structure. Consumption of two to three grams per day of plant stanols is well-known to interfere with intestinal cholesterol absorption, thereby lowering plasma cholesterol levels and as such reducing inflammatory cascades (3). In addition, studies suggest that plant stanols also have immunomodulatory and antiinflammatory effects $(4,5)$, and it has been suggested that these effects might occur independently from their ability to reduce cholesterol absorption (4). However, this assumption of independency has yet to be validated in in vivo models for metabolic diseases. Previously, our group showed that dietary plant stanol supplementation reduces hepatic cholesterol levels, ameliorates liver inflammation, and shifts blood immune cells towards a less pro-inflammatory profile in a murine model of Niemann-Pick type C1 (NPC1) disease (5). NPC1 disease is caused by deleterious mutations in the NPC1 gene that lead to the production of a defective NPC1 protein, a lysosomal cholesterol efflux transporter (6). Following impaired NPC1 protein function, lysosomal cholesterol and sphingolipid accumulation occurs in all tissues, culminating in increased oxidative stress and severe inflammation. Importantly, in the aforementioned study (5), mice were fed a diet with much lower cholesterol content than in an experimental high fat, high cholesterol (HFC) diet $(0.37 \mu \mathrm{g}$ vs $1.62 \mu \mathrm{g}$ cholesterol $/ \mathrm{mg}$ chow on average). As such, this study puts forward the hypothesis that plant stanols have anti-inflammatory effects independent of reduced cholesterol intestinal absorption, although such effects have not been evaluated yet side-by-side on a background of either cholesterol-rich or cholesterol-poor diets. 
Therefore, in the current study, our aim was to investigate to what extent the effects of dietary plant stanol supplementation on hepatic and systemic cholesterol metabolism and inflammation depend on dietary cholesterol intake. To this end, we analyzed the effects of two-percent dietary plant stanol supplementation on either an HFC or a chow diet in a model for cholesterol-induced inflammation. Here, we used low-density lipoprotein receptor knockout ( $\left(\mathrm{dll}^{-/-}\right)$mice, which, when fed an HFC diet, display hypercholesterolemia and increased hepatic cholesterol accumulation and inflammation, thus mimicking the human situation of metabolic diseases such as atherosclerosis and non-alcoholic steatohepatitis $(7,8)$. In order to induce hepatic lipid accumulation and inflammation in the absence of a HFC diet, we transplanted $L d l r^{-1-}$ mice with $N p c 1^{\text {nih }}$ bone marrow (Npc1 $1^{\text {nih }}$-tp mice) (9). These $N p c 1^{\text {nih }}$-tp mice display increased levels of hepatic inflammation compared to their Npc1wt-tp counterparts, even in the absence of a HFC diet, thus serving as a model to investigate the influence of diet on the anti-inflammatory properties of plant stanols. 


\section{MATERIALS AND METHODS}

\section{Mice, Bone Marrow Transplant, and Diet}

Throughout the study, mice were housed under standard conditions and had unlimited access to food and water, unless explicitly mentioned otherwise. For one week prior to and up to four weeks after irradiation, $L d l r^{-1-}$ mice were housed in filter-top cages and received antibiotics diluted in drinking water to prevent infections following immunosuppression (Neomycin, $100 \mathrm{mg} / \mathrm{L}$, Gibco, Breda, the Netherlands; $6 \times 10^{4} \mathrm{U} / \mathrm{L}$ polymycin B sulphate). Sixweek-old bone marrow from $N p c 1^{\text {nih }}$ and $N p c 1^{w t}$ mice donors were derived from heterozygous founders of a C57BL/6 genetic background. The genotype of $N p c 1^{\text {inh }}$ and $N p c 1^{w t}$ mice was determined as previously described (10). On the day of bone marrow transplant, $N p c 1^{\text {nih }}$ and $N p c 1^{w t}$ littermates were sacrificed via $\mathrm{CO}_{2}$ inhalation and their bone marrows were isolated. On the day before and on the day of bone marrow transplant, $\mathrm{Ldll}^{-1}$ mice were subjected to six Gray of $\gamma$-radiation, thus having received 12 Gray of $\gamma$-radiation before receiving $1 \times 10^{7}$ bone marrow cells collected from $N p c 1^{w t}$ or $N p c 1^{\text {nih }}$ mice via intravenous injection.

After nine weeks of recovery, transplanted mice were placed on a chow or HFC diet for twelve weeks, after which they were assigned to two different experimental groups and received the corresponding plant stanol experimental diets (Table S1) for three weeks, for a total of eight experimental groups ( $n=6-8$ mice per group): (1) $N p c 1^{\text {wt }}$-tp and $N p c 1^{\text {nih }}$-tp mice on a regular chow diet; (2) $N p c 1^{w t}$-tp and $N p c 1^{\text {nih }}$-tp mice on a two percent plant stanol-enriched chow diet; (3) $N p c 1^{w t_{-} \text {-tp }}$ and $N p c 1^{\text {nih }}$-tp mice on a HFC diet; (4) $N p c 1^{\text {wt }}$-tp and $N p c 1^{\text {in }}{ }_{\text {-tp }}$ mice on a two percent plant stanol-enriched HFC diet. For a schematic overview of the study setup and timeline, please refer to Figure 1. All experiments were performed according to Dutch laws and approved by the Animal Experiment Committee of Maastricht University (study DEC 2013-002, approved on 19/03/2013).

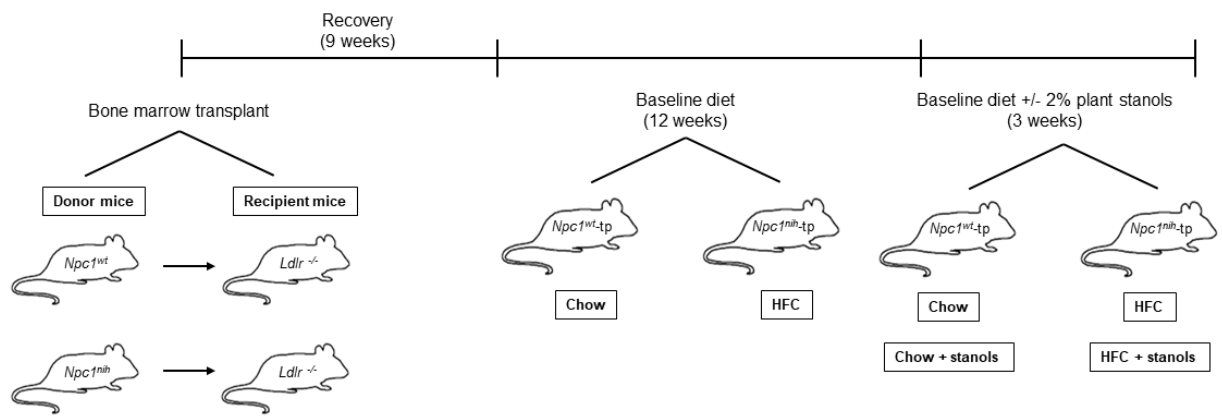

Figure 1. Overview of study setup and timeline. $L d l r^{-1}$ mice received a bone marrow transplant from $N p c 1^{\text {nih }}$ or $N p c 1^{w t}$ mice. After recovery, $N p c 1^{\text {wt-tp }}$ and $N p c 1^{\text {nih }}$-tp mice received a cholesterol-poor chow or HFC diet for 12 weeks. On the final three weeks of the study period, their diet was supplemented with $2 \%$ plant stanols. 
Bone Marrow Transplant Efficiency

In order to assess bone marrow transplant efficiency, we determined chimerism in $\mathrm{Ldlr}^{-1-}$ mice transplanted with bone marrow from Ldlrwt mice. Genomic DNA was isolated using the PureLink Genomic DNA, according to the manufacturer's instructions (K182002; ThermoFisher Scientific, Waltham, Massachusetts, U.S.A.). A standard curve was built by producing solutions with different ratios of $L d l r^{-}$-and $L d l r^{w t}$ bone marrow DNA. To assess chimerism of mice in this study, we analyzed the amount of $L d l r^{-1}$ DNA in $70 \mu \mathrm{L}$ peripheral blood samples. To standardize the total amount of DNA among different samples, the $p 50$ gene expression was quantified. Samples were assayed in duplicate on a 7900HT real-time PCR system by using 25 ng DNA and a SensiMixTM Sybr \& Fluorescein kit (QT615-05, Bioline, Memphis, Tennessee, U.S.A.), according to the manufacturer's instructions. Ldlr ${ }^{-1}$-specific primers are forward 5'- GCTGCAACTCATCCATATGCA-3' and reverse 5'GGAGTTGTTGACCTCGACTCTAGAG-3'. Forward and reverse $p 50$-specific primers are $5^{\prime}$ ACCTGGGAATACTTCATGTGACTAA-3' and 5'-ACACCAGAAGTCCAGGATTATCAG3', respectively. A standard curve was generated by plotting the mean threshold cycle (Ct) $\Delta \mathrm{Ct}(\mathrm{Ct} p 50-\mathrm{Ct}$ $L d l r^{-}$) against the logarithm of the percentage $L d l r^{--}$and calculation of a regression line. Bone marrow transplant efficiency was determined as the percentage of Ldlr ${ }^{-1}$ DNA in the mice blood samples (representing the remaining recipient bone marrow), calculated by applying the mean $\Delta \mathrm{Ct}$ of the sample to the previously generated standard curve. On average, bone marrow transplantation was nearly $94 \%$ efficient (Table S2), indicating that the procedure was successful.

\section{Lipid and Gene Expression Analyses}

Upon sacrifice, all tissues were isolated and snap-frozen in liquid nitrogen and stored at $-80^{\circ} \mathrm{C}$ or fixed in $4 \%$ formaldehyde/PBS. The collection of blood and tissue specimens, biochemical determination of lipids in plasma, RNA isolation, cDNA synthesis, and qPCR were determined as described previously (11-13). Hepatic sterol content was determined by gas-liquid chromatography-mass spectroscopy, as described elsewhere (14).

\section{Immunohistochemistry}

Frozen liver sections $(7 \mu \mathrm{m})$ were fixed in acetone and blocked for endogenous peroxidase by incubation with $0.25 \%$ of $0.03 \% \mathrm{H}_{2} \mathrm{O}_{2}$ for $5 \mathrm{~min}$. Primary antibodies used were against hepatic macrophages (1:100 rat anti-mouse CD68, clone FA11), infiltrated macrophages and neutrophils (1:500 rat anti-mouse Mac-1 (M1/70)]), and infiltrated T-cells (1:20, rat anti-mouse CD3). 3-Amino-9-ethylcarbazole was applied as color substrate and hematoxylin for nuclear counterstain. Sections were enclosed with Faramount aqueous mounting medium.

Pictures were taken with a Nikon digital camera DMX1200 and ACT-1 v2.63 software (Nikon Instruments Europe, Amstelveen, The Netherlands). Infiltrated macrophages and neutrophil cells (Mac-1+) and infiltrated T-cells (CD3+) were counted by two blinded researchers in six microscopical views (original magnification, 200x) and were indicated as number of cells per square millimeter (cells $/ \mathrm{mm}^{2}$ ). Hepatic macrophages (CD68) were counted in six microscopical views (original magnification, 200x) and indicated as the percentage of CD68 positive area (Adobe Photoshop CS2 v.9.0., San Jose, California, U.S.A.). 
Plasma FACS Analyses

Tail vein blood was collected from mice 12 and 15 days after the beginning of the plant stanol-enriched diet. FACS procedures were performed as previously described (5).

\section{Statistical Analysis}

Data were statistically analyzed by performing two-way ANOVA and Tukey's post hoc test using GraphPad Prism software (version 6 for Windows, GraphPad Software Inc, San Diego, California, U.S.A.). Data were expressed as the group mean and standard error of the mean. Statistical significance is indicated on the following data comparisons: $N p c 1^{\text {wt }}$-tp or $N p c 1^{\text {nih }}$-tp mice on a chow or HFC diet vs $N p c 1^{w t-t p}$ or Npc1 ${ }^{\text {nih-tp }}$ mice on a plant stanol-enriched chow or HFC $\operatorname{diet}\left({ }^{*} p \leq 0.05 ;{ }^{* *} p<0.01 ;{ }^{* * *} p<\right.$ 0.001; $\left.{ }^{* * * *} p<0.0001\right) . N p c 1^{\text {nih }}$-tp mice on a chow or HFC diet vs Npc1 $1^{\text {wt }}$-tp mice on a chow or HFC diet (\# $p \leq 0.05 ; \# p<0.01$; \#\#\# $p<0.001$; \#\#\# $p<0.0001)$. 


\section{RESULTS}

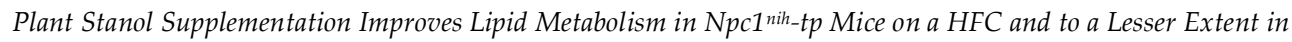
Mice on a Chow Diet

To determine to what extent the effects of plant stanols on lipid metabolism are dependent on diet, we analyzed plasma and liver cholesterol levels of $N p c 1^{\text {wt }}$-tp mice and of $N p c 1^{\text {nih }}$-tp mice placed on an HFC or chow diet for 15 weeks. At baseline, $N p c 1^{\text {nih }}$-tp mice displayed lower plasma total cholesterol but higher hepatic cholesterol concentrations as compared to the Npc1 ${ }^{w t}$-tp mice, both following HFC and chow diets (Figure 2).

As expected, adding plant stanols to the HFC diet effectively reduced plasma and hepatic cholesterol levels in both experimental groups (Figure 2), whereas plant stanol supplementation of the chow diet had no significant effect on plasma and liver cholesterol levels in either experimental group (Figure 2).

A

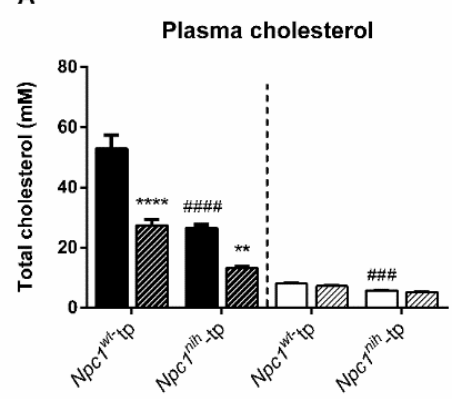

B

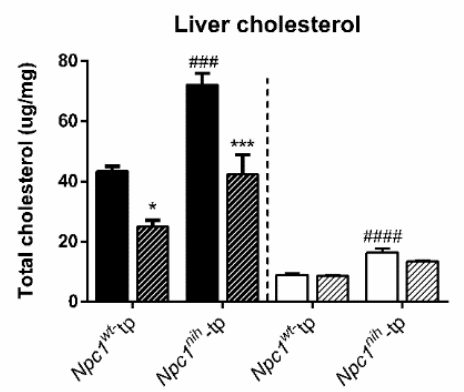

HFC

WIII HFC + stanol

$\square$ Chow

WIIIA Chow + stanol

Figure 2. Plasma and liver lipid levels: HFC and chow diets. (A) Total plasma cholesterol levels and (B) total liver cholesterol levels. $n=6-8$ mice per group. All error bars represent standard error of the mean. Statistical significance is indicated as follows: $N p c 1^{\text {nih }}$-tp mice on an HFC or chow diet vs. $N p c 1^{w t-t p}$ mice on an HFC or chow $\operatorname{diet}$ (\#\#\# $p<0.001$; \#\#\# $p<0.0001) ; N p c 1^{w t}$-tp or $N p c 1^{n i h_{-}}$-tp on a HFC or chow diet vs. $N p c 1^{w t}$-tp or $N p c 1^{\text {nih }}$-tp mice on a plant stanolenriched HFC or chow $\operatorname{diet}\left({ }^{*} p \leq 0.05 ;{ }^{* *} p<0.01 ;{ }^{* * *} p<0.001 ;{ }^{* * * *} p<0.0001\right)$.

To have a deeper insight into hepatic cholesterol metabolism following plant stanol supplementation, we assessed liver levels of the following sterols in relation to liver cholesterol levels: sitostanol and campestanol, which were included in the plant stanolsupplemented diet and can be seen as compliance markers; desmosterol, a cholesterol biosynthesis precursor; and 27-OH cholesterol, a bile acid precursor. After three weeks of plant stanol supplementation to HFC or chow diet, relative hepatic sitostanol and campestanol levels increased in both chimeric groups, as expected (Figure 3A,B). Following an HFC diet, increased relative desmosterol levels and reduced 27-OH cholesterol were observed in $N p c 1^{\text {nih }}$-tp mice compared to $N p c 1^{w t}$-tp mice (Figure 3C,D). Regarding the chow diet setting, relative liver desmosterol levels were also increased in $N p c 1^{\text {nih }}$-tp mice compared to $N p c 1^{w t}$-tp mice, whereas relative $27-\mathrm{OH}$ cholesterol levels tended to be reduced in $N p c 1^{\text {iih }}$ tp mice compared to $N p c 1^{w t}$-tp mice (Figure 3C,D). 
While supplementing the HFC diet with plant stanols had no effect on relative desmosterol levels in the livers of $N p c 1^{\text {wt }}$-tp mice, $N p c 1^{\text {nih }}$-tp mice displayed increased relative desmosterol levels following increased plant stanol intake (Figure 3C). The aforementioned results suggest that, following a HFC diet, plant stanol supplementation increases liver cholesterol synthesis in $N p c 1^{\text {nih }}$-tp mice, but not in $N p c 1^{\text {wt }}$-tp mice. Furthermore, plant stanol supplementation to an HFC diet increased relative liver levels of $27-\mathrm{OH}$ cholesterol in both chimeric groups, suggesting increased metabolism of cholesterol to oxidized sterols acids following increased plant stanol intake (Figure 3D). In contrast to the HFC setting, plant supplementation to a chow diet had no effects on hepatic levels of desmosterol and 27-OH cholesterol in either chimeric group (Figure 3D).

A

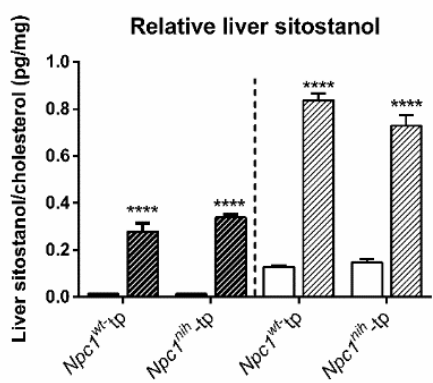

C

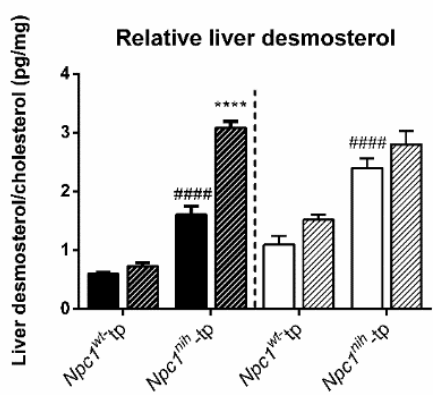

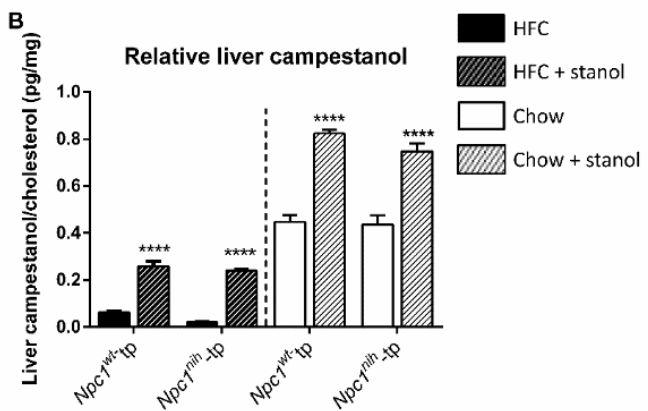

D

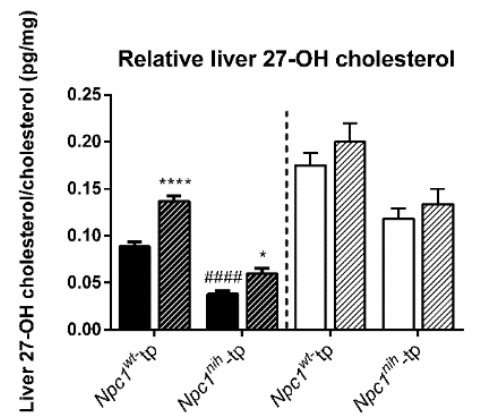

Figure 3. Hepatic sterol lipid metabolism: HFC and chow diets. Liver levels of (A) sitostanol, (B) campestanol, (C) desmosterol, and (D) 27-OH cholesterol relative to total hepatic cholesterol levels. $n=6-8$ mice per group. All error bars represent standard error of the mean. Statistical significance is indicated as follows: $N p c 1^{\text {in }}$-t $p$ mice on an HFC or chow diet vs $N p c 1^{w t_{-}} t p$ mice on a HFC or chow diet (\#\#\# $\left.p<0.0001\right)$; $N p c 1^{w t_{-}}$-tp or $N p c 1^{\text {nih }}$-tp on a HFC or chow diet vs $N p c 1^{w t_{-}}$ tp or $N p c 1^{\text {nih }}$-tp mice on a plant stanol-enriched HFC or chow $\operatorname{diet}\left({ }^{*} p \leq 0.05\right.$; $\left.{ }^{* * *} p<0.0001\right)$.

To further assess the effects of plant stanol supplementation in liver lipid metabolism, we analyzed the liver expression of the following genes: $N p c 2$, which encodes for a protein that transfers luminal cholesterol in late endosomes/lysosomes to NPC1; Cd36, a receptor for modified LDL, a pro-inflammatory lipoprotein; Abca1 and Abcg1, which mediate HDL synthesis and excess cholesterol efflux; Cyp7a1, a bile acid synthesis mediator; and Osbpl1, a 
cytosolic protein that binds oxygenated forms of cholesterol (Figure 4). Following an HFC diet, $N p c 1^{\text {nih }}$-tp mice displayed higher $N p c 2$ and $A b c g 1$ expression but lower Osbpl1 expression as compared to the $N p c 1^{w t}$-tp mice (Figure 4A,D,F). Regarding the chow diet setting, while expression of most analyzed genes was comparable between the experimental groups, hepatic $N p c 2$ expression tended to be increased in $N p c 1^{\text {nih }}$-tp mice, whereas Osbpl1 expression was effectively reduced in $N p c 1^{\text {nih }}$-tp mice compared to $N p c 1^{w t}$-tp mice (Figure $4 \mathrm{~F}$ ).

A
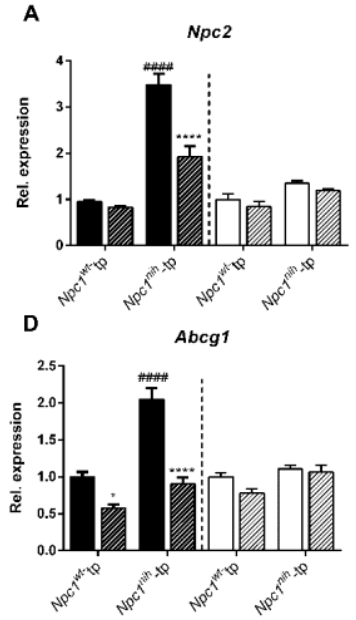

B
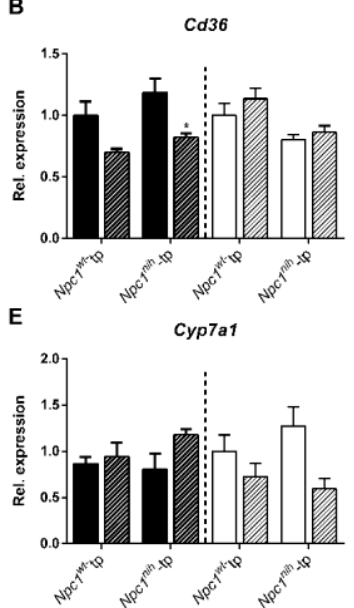
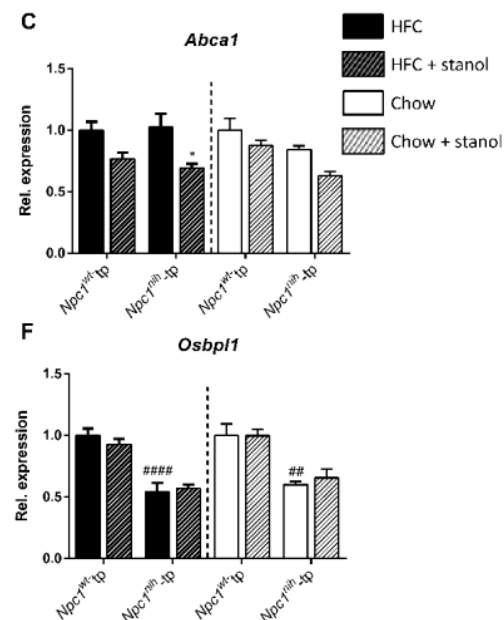

Figure 4. Hepatic lipid metabolism-related gene expression: HFC and chow diets. Hepatic gene expression levels of (A) Npc2, (B) Cd36, (C) Abca1, (D) Abcg1, (E) Cyp7a1, and (F) Osbpl1. n=6-8 mice per group. All error bars represent standard error of the mean. Statistical significance is indicated as follows: $N p c 1^{w t-t p}$ mice on a HFC or chow diet vs. Npc1wt-tp mice

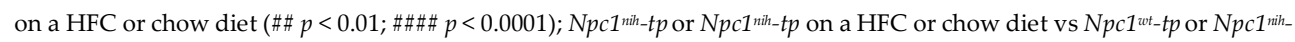
$t p$ mice on a plant stanol-enriched HFC or chow $\operatorname{diet}\left({ }^{*} p \leq 0.05 ;{ }^{* * * *} p<0.0001\right)$.

Adding plant stanols to the HFC diet reduced Npc2, Cd36, Abca1, and Abcg1 hepatic expression in $N p c 1^{\text {nih }}$-tp mice, suggesting reduced lysosomal lipid accumulation, modified cholesterol uptake, and excess cholesterol efflux (Figure 4A-D). Furthermore, increased plant stanol consumption also reduced $A b c g 1$ expression and tended to reduce hepatic $C d 36$ expression in the livers of $N p c 1^{\text {wt }}$-tp mice on a HFC diet, although the latter effect did not reach statistical significance (Figure 4B,D). Following plant stanol supplementation to the chow diet, no significant differences were observed in any of the analyzed hepatic genes' expression. Altogether, the results described above indicate that dietary plant stanol supplementation only has minor beneficial effects on lipid metabolism in the absence of a HFC diet in $N p c 1^{\text {nih }}$-tp mice. 
Dietary Plant Stanol Supplementation Has Little Impact on Liver Inflammation in the Absence of a HFC Diet

To investigate whether plant stanol supplementation reduces liver inflammation in the absence of a beneficial plant stanol-induced metabolic effect, we measured hepatic macrophages $\left(\mathrm{CD}^{+} 8^{+}\right)$and infiltrated macrophages and neutrophils (Mac- $\left.1^{+}\right)$, as well as infiltrated T-cells $\left(\mathrm{CD}^{+}\right)$via immunohistochemistry (Figure 5). Following an HFC diet, $N p c 1^{\text {nih }}$-tp mice displayed higher numbers of hepatic macrophages, infiltrated macrophages, neutrophils, and T-cells as compared to the $N p c 1^{w t}$-tp mice (Figure 5). Regarding the chow diet setting, while no differences were observed regarding infiltrated macrophages and neutrophils between experimental groups, $N p c 1^{{ }^{\text {nih }} \text {-tp }}$ mice displayed increased presence of hepatic macrophages and infiltrated T-cells compared to $N p c 1^{w t-t p}$ mice, suggesting increased liver inflammation following $\mathrm{Npc1}$ mutation in bone marrow-derived immune cells (Figure $5)$.

As expected, while plant stanol supplementation to HFC diet had no effect on hepatic levels of immune cells in Npc1 ${ }^{\text {wt }}$-tp mice, dietary plant stanol supplementation reduced the amount of hepatic macrophages and infiltrated macrophages and neutrophils in Npc1 ${ }^{\text {nih }}$-tp mice (Figure 5). In addition, Npc1 ${ }^{\text {nih }}$-tp mice on a plant stanol-supplemented HFC diet showed a tendency for reduced infiltrated T-cells, although this effect did not reach statistical significance (Figure 5C). Supplementing the mice's chow diet with plant stanols had no effect on the hepatic levels of any of the immune cells in either experimental group (Figure 5).

To profile the effects of plant stanols on hepatic inflammation in more detail, we analyzed the expression of the following genes: Tnfa, Cd68, Ccl2, Il12, Caspase1, Ctsd, Icam, and Vcam (Figure 6). Following an HFC diet, Npc1 $1^{\text {nih-tp }}$ mice displayed increased expression of Cd68, Il12, Caspase1, Ctsd, and Icam compared to Npc1wt-tp mice (Figure 6B,D-G). In addition, Ccl2 expression tended to be increased in $N p c 1^{\text {nih }}$-tp mice compared to $N p c 1^{w t-t p}$ mice, although this effect did not reach statistical significance (Figure 6C). Regarding the chow setting, $\mathrm{Npc}^{\text {nih }}$-tp mice on a chow diet displayed increased hepatic expression of $\mathrm{Cd} 68$, Ctsd, and Vcam compared to $N p c 1^{w t}$-tp mice (Figure $6 \mathrm{~B}, \mathrm{~F}, \mathrm{H}$ ). In line with our immunohistochemical observations, these results indicate increased hepatic inflammation in $N p c 1^{\text {nih }}$-tp mice compared to Npc1 ${ }^{w t}$-tp mice, particularly in the HFC diet setting. 

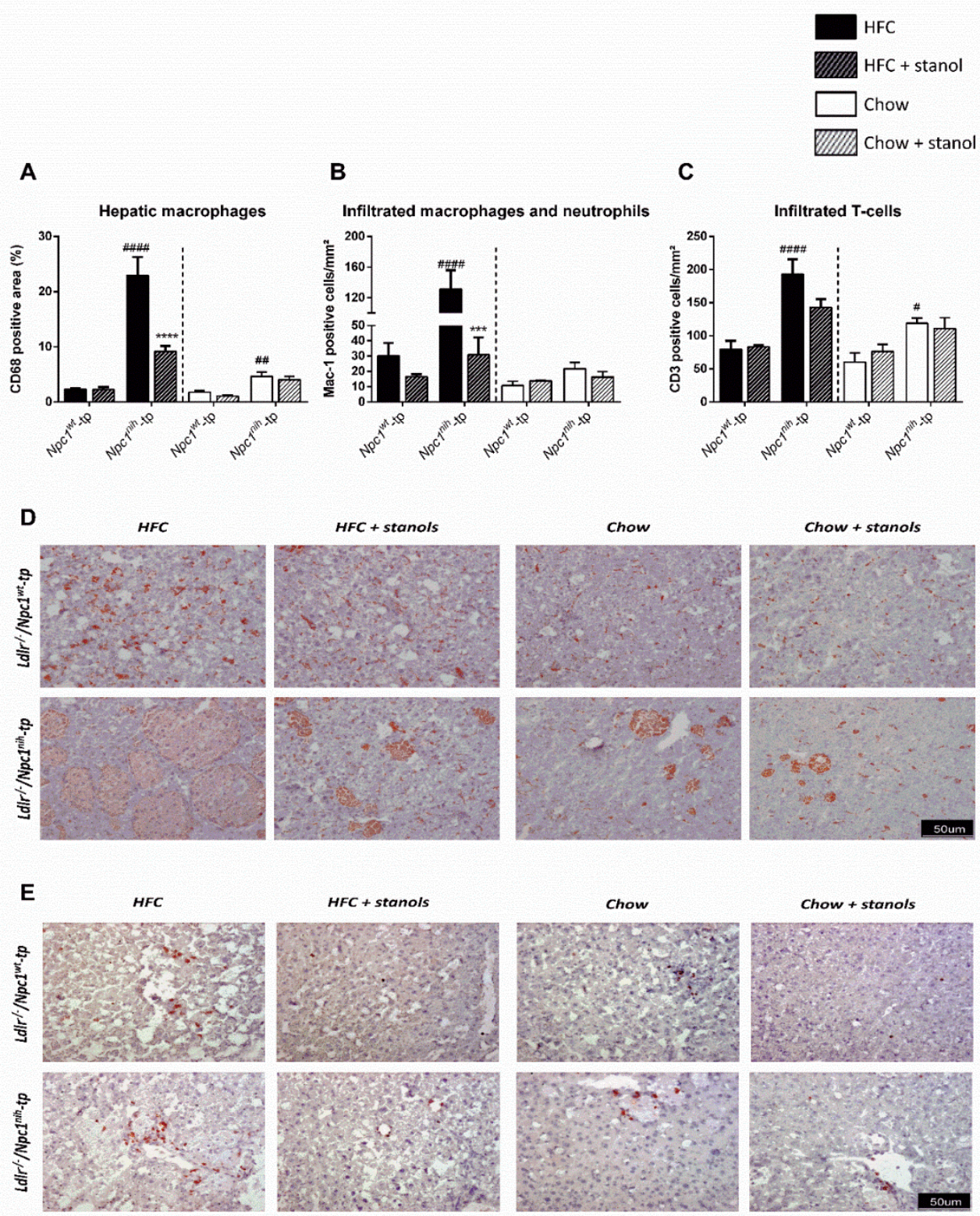

Figure 5. Hepatic inflammation-related immunohistochemistry: HFC and chow diets. (A-C) Quantification of hepatic macrophages (CD68), infiltrated hepatic macrophages and neutrophils (Mac-1), and infiltrated hepatic T-cells (CD3). (D-E) Representative pictures of liver sections stained for hepatic macrophages and infiltrated macrophages. All error bars represent standard error of the mean. Statistical significance is indicated as follows: $N p c 1^{\text {inh }}-t p$ mice on a HFC or

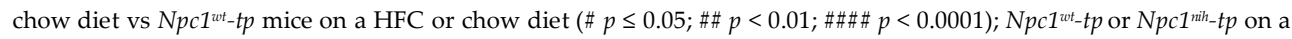
HFC or chow diet vs Npc1 $1^{\text {wt }}$-tp or Npc1 $1^{\text {nih }}$-tp mice on a plant stanol-enriched HFC or chow $\operatorname{diet}\left(^{* * *} p<0.001\right.$; $^{* * * *} p<$ $0.0001)$. 
Following plant stanol supplementation to the HFC diet, expression of Tnfo and Ccl2 was reduced in both experimental groups (Figure 6A,C). Furthermore, $N p c 1^{\text {nih-tp }}$ mice displayed reduced expression of Cd68, Caspase1, and Ctsd, as well as a tendency for reduced Vcam expression, following a plant stanol-enriched HFC diet (Figure 6B,E,F,H). Finally, we observed reduced expression of Vcam in the livers of $N p c 1^{\text {wt }}$-tp mice on a plant stanol-enriched HFC diet compared to Npc1wt-tp mice on a HFC diet (Figure 6H). Similarly to immunohistochemistry results, plant stanol supplementation to the chow diet had no impact on the expression of the analyzed genes in either genotype (Figure 6). Overall, the results described above indicate that, in line with lipid metabolism results, plant stanol supplementation had fewer effects on liver inflammation in Npc1 ${ }^{\text {in }}$-tp mice fed a chow diet compared to $N p c 1^{\text {nih }}$-tp mice fed a HFC diet.
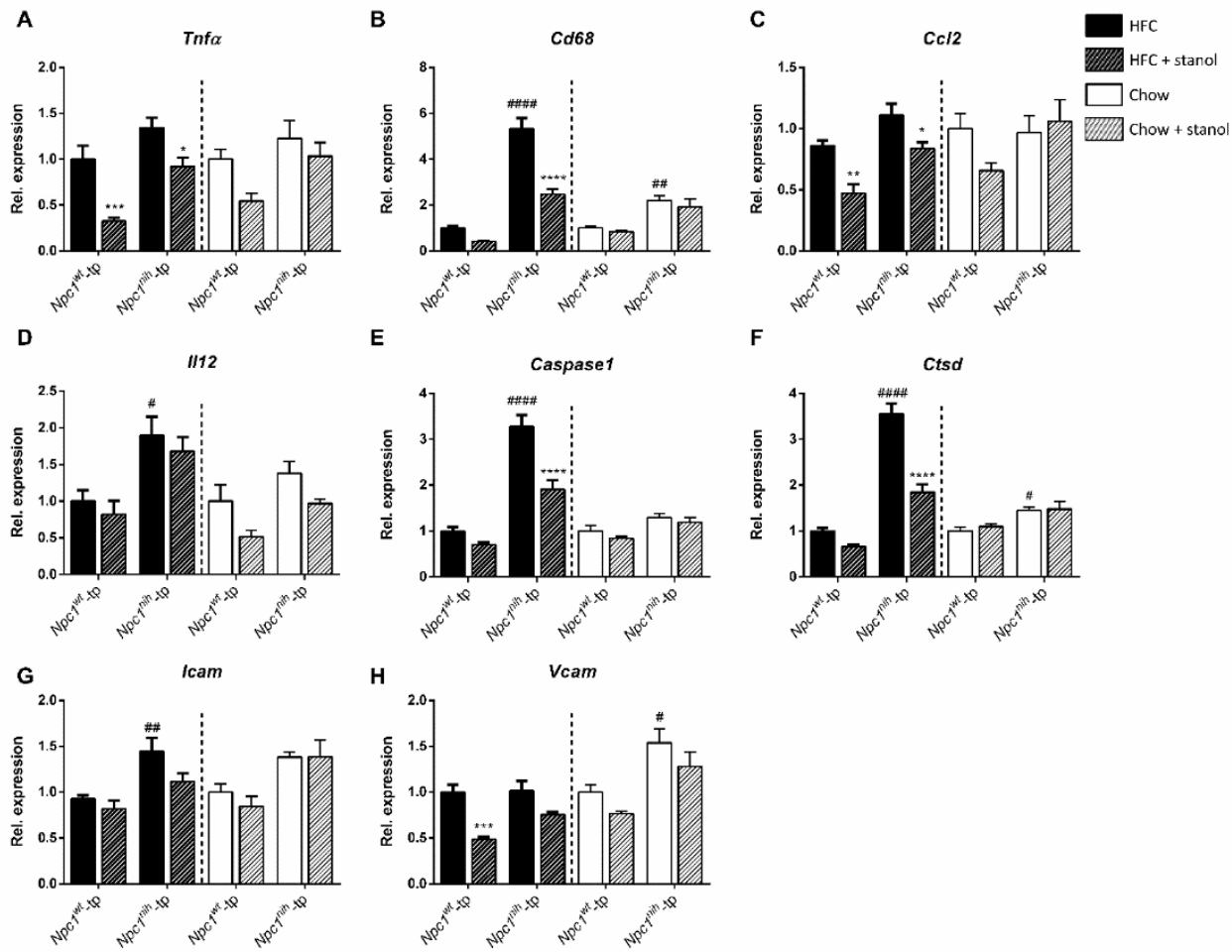

Figure 6. Hepatic inflammation-related gene expression: HFC and chow diets. Hepatic gene expression of inflammatory markers (A) Tnf- $\alpha$, (B) Cd68, (C) Ccl2, (D) Il12, (E) Caspase1, (F) Ctsd, (G) Icam and (H) Vcam. n= 6-8 mice per group for gene expression analyses. All error bars represent standard error of the mean. Statistical significance is indicated as follows: Npc1 $1^{\text {nih }}$-tp mice on a HFC or chow diet vs Npc1 $1^{w t}$-tp mice on a HFC or chow diet $(\# p \leq 0.05 ; \# \#<0.01$; \#\#\#p<

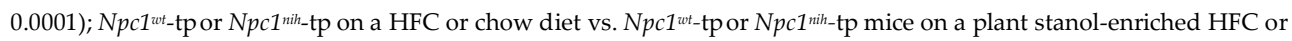
chow $\operatorname{diet}\left({ }^{*} p \leq 0.05 ;{ }^{* *} p<0.01 ;{ }^{* * *} p<0.001 ;{ }^{* * * *} p<0.0001\right)$. 
Finally, we analyzed the blood monocytes and T-cells three weeks after the start of plant stanol supplementation to further investigate the effects of plant stanol supplementation on systemic inflammation (Figure 7). In this study, we analyzed the relative amounts of blood $\mathrm{LyC6}^{+}$cells (a marker for activated pro-inflammatory monocytes), as well as of CD4 ${ }^{+}$and CD8 ${ }^{+}$ cells, which denote helper and cytotoxic T-cells, respectively. While no differences were observed on T-cell populations among genotypes following an HFC diet (Figure 7C,D), $N p c 1^{\text {nih }}$-tp mice displayed lower levels of anti-inflammatory LyC6 $6^{\text {low }}$ monocytes and higher levels of pro-inflammatory LyC6 $6^{\text {high }}$ monocytes than $N p c 1^{w t}$-tp mice, indicating increased systemic inflammation in the former genotype following an HFC diet (Figure 7A,B). Regarding the chow setting, although $N p c 1^{\text {nih }}$-tp mice displayed reduced relative levels of blood T-helper cells compared to $N p c 1^{w t}$-tp mice (Figure 7C), relative amounts of blood antiand pro-inflammatory monocytes and cytotoxic T-cells were comparable between chimeric groups following a chow diet (Figure 7A,B,D).

Following plant stanol enrichment to the HFC diet, no significant effects were observed on any of the analyzed blood immune cell populations in either genotype (Figure 7). Of note, plant stanol supplementation to the HFC diet tended to increase levels of anti-inflammatory monocytes and to reduce levels of pro-inflammatory monocytes in $N p c 1^{\text {nih }}$-tp mice, although this effect did not reach statistical significance. After three weeks of plant stanol supplementation to the chow diet, we observed increased amounts of CD4+ T-cells in Npc1 ${ }^{\text {nih- }}$ tp mice, suggesting that plant stanols increased the relative amount of blood helper T-cells (Figure 7C). Overall, despite the observed increase in helper T-cell levels in Npc1 $1^{\text {nih }}$-tp mice on a plant stanol-enriched chow diet, these results suggest that three weeks of plant stanol supplementation has little impact on blood immune cell populations, regardless of genotype or diet. 
A
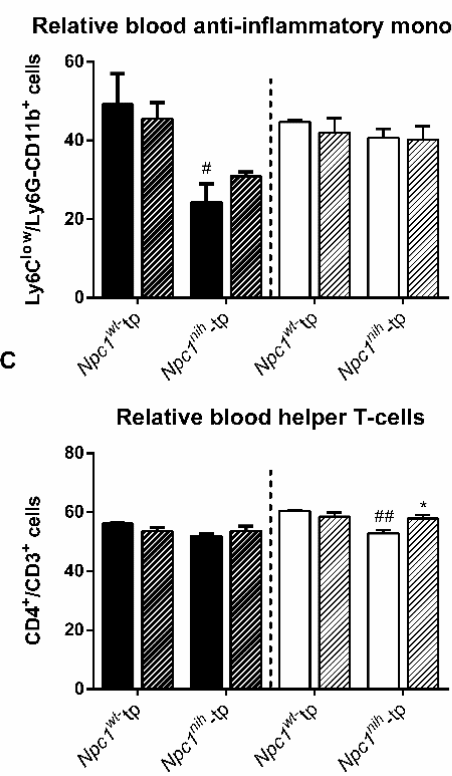

B

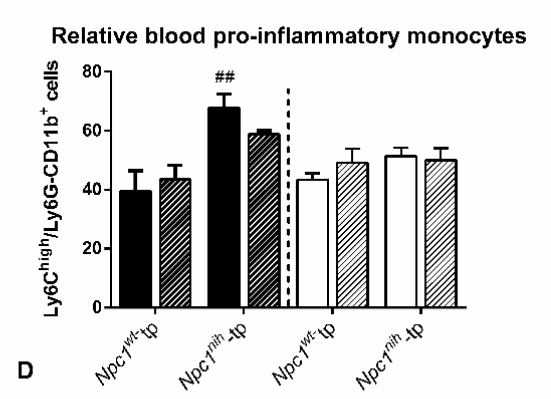

HFC
HFC + stanol
Chow
Chow + stanol

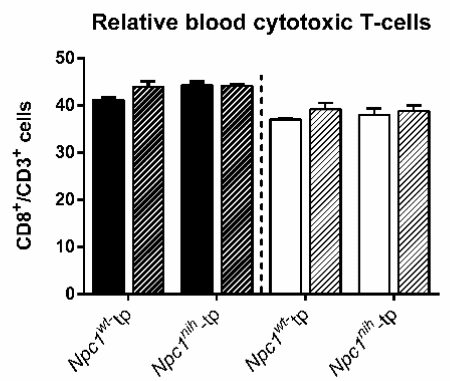

Figure 7. Phenotype and levels of blood monocytes and T-cells: HFC and chow diets. Relative levels of plasma (A) proinflammatory (LyC6+) and (B) anti-inflammatory (LyC6-) monocytes, as well as of (C) cytotoxic (CD8+) and (D) helper T-cells (CD4+) were measured by FACS analysis. $n=4$ mice per group. All error bars represent standard error of the mean. Statistical significance is indicated as follows: Npc1 $1^{\text {ih }}$-t $p$ mice on a HFC or chow diet vs. Npc1 $1^{w t-t p}$ mice on a HFC

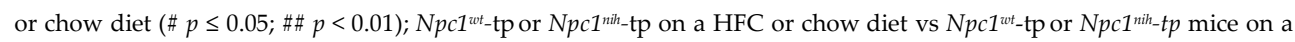
plant stanol-enriched HFC or chow diet $\left.{ }^{*} p \leq 0.05\right)$.

Altogether, the results described in this study indicate that beneficial effects of dietary plant stanol supplementation on lipid metabolism and inflammation are mostly dependent on excess intake of cholesterol in the analyzed model. 


\section{DISCUSSION}

Although glucose and lipid metabolism dysregulation are at the core of the metabolic syndrome, the ensuing inflammatory response underlies the development of metabolic syndrome-related diseases, such as atherosclerosis and NASH. As such, tools to prevent or reduce inflammation are paramount for metabolic syndrome patients. Plant stanols are wellknown to reduce dietary cholesterol absorption and to consequently reduce plasma cholesterol levels and inflammation. However, reports also suggest that plant stanols may have direct anti-inflammatory effects of their own, increasing their appeal as affordable, easily accessible anti-inflammatory tools for individuals with inflammatory disorders who do not consume cholesterol in excess. In this study, only subtle trends towards improved metabolism and inflammation were observed following plant stanol supplementation to a cholesterolpoor diet. In contrast, plant stanol supplementation to the HFC diet clearly improved lipid metabolism and inflammation in $N p c 1^{\text {nih }}{ }_{\text {-tp }}$ mice. Our results suggest that individuals who consume excessive amounts of cholesterol are likely to benefit the most from plant stanol supplementation.

For decades, plant stanols and their unsaturated analogues, plant sterols, have been known to interfere with intestinal cholesterol absorption and to reduce plasma cholesterol levels in animal models and humans alike. Consequently, a variety of plant stanol-supplemented foods have been developed, which are often recommended to (mildly) hypercholesterolemic patients as a tool to reduce their plasma cholesterol levels. While the mechanisms underlying the effects of plant stanols on intestinal cholesterol absorption are still a matter of debate, it is likely that this effect is accomplished by a variety of processes, including interference with chylomicron cholesterol incorporation and stimulation of intestinal cholesterol excretion, for instance via LXR activation (3). In addition to stimulating cholesterol excretion in the intestines, it is possible that absorbed plant stanol molecules can likewise activate LXR signaling in the liver, thereby modulating hepatic lipid metabolism even in the absence of an HFC diet. Of note, experimental manipulations that were used in this experiment likely did not change intestinal NPC1L1 expression, excluding a role for this transporter in our study (3). While our results strengthen the view that plant stanols' effects on hepatic lipid metabolism are highly dependent on interference with intestinal cholesterol absorption, the aforementioned trends suggest that plant stanol molecules may have direct effects on hepatic lipid metabolism, for instance via LXR activation. Since only about $0.15 \%$ of ingested plant stanols is effectively absorbed into the circulation (15), it is possible that such direct effects would have translated into stronger modulation of hepatic lipid metabolism following supplementation with increased concentration of plant stanols to the chow diet during a longer time period. In addition to being well-known LXR activators, plant stanols also 
modulate sterol metabolism, which ultimately can influence hepatic inflammation. Relevantly, while in a previous study plant stanol supplementation reduced liver desmosterol levels in $\mathrm{Ldll}^{-}$- mice fed a HFC diet (16), here we observed a further increase in liver desmosterol levels of $N p c 1^{\text {nih }}$-tp mice following plant stanol supplementation to an HFC diet, and a similar trend was observed in the regular chow diet setting. While the physiological relevance of such an increase is unclear, considering the accompanying reduction in liver inflammation, it is possible that plant stanol supplementation stimulates $N p c 1^{\text {iih }}$ macrophages to further accumulate desmosterol. Such a desmosterol accumulation could increase LXR activation and inhibit SREBP-pathways, thereby reducing the inflammatory profile of Npc1 ${ }^{\text {ih }}$ macrophages and possibly contributing to reduced inflammation following plant stanol supplementation $(17,18)$.

In addition to interfering with intestinal cholesterol absorption and modulating lipid metabolism via diverse signaling pathways, there is further evidence indicating that plant stanols may have anti-inflammatory and immunomodulatory effects independent from their effects on cholesterol intestinal absorption. Previously, one in vitro study showed that sitostanol administration to bone marrow-derived macrophages reduced TNF $\alpha$ secretion in the absence of changes in Lxr expression (16), suggesting that plant stanols shift macrophages towards a less pro-inflammatory profile independently of LXR activation and of lipid metabolism status of other cell types, such as hepatocytes. Further studies showed that increased plant stanol consumption shifts T-cells towards a Th1 profile in asthma patients, a process likely mediated by TLR2 activation (19-21). These findings suggest that plant stanols have immunomodulatory effects independent of effects on lipid metabolism. However, such effects have yet to be validated in in vivo models regarding metabolic inflammatory disorders, such as NASH, cardiovascular disease, and atherosclerosis. In the current study, apart from a modest increase in circulating helper T-cells in Npc1 ${ }^{\text {nih }}$-tp mice, we observed only subtle reductions in hepatic inflammatory markers following plant stanol supplementation to a chow diet. It should be noted that, while $N p c 1^{\text {nih }}$-tp mice consistently displayed higher liver lipid accumulation and inflammation compared with $N p c 1^{w t}$-tp mice, the disease phenotype was much more subtle in $N p c 1^{\text {nih }}$-tp mice fed a chow diet than in the HFC diet setting. As such, it is possible that a stronger disease phenotype is needed in order to observe more pronounced effects of plant stanol supplementation. In addition, in a previous study (5), Npc1 $1^{\text {nih }}$ mice on a cholesterol poor diet showed strong improvements in systemic inflammation after five weeks of plant stanol supplementation, rather than three, as in the current study. Furthermore, while we observed improvements in the NPC1 disease phenotype following $2 \%$ plant stanol supplementation in this study (5), such effects were more pronounced in mice consuming a $6 \%$ plant stanol-enriched diet. Therefore, it is possible that administration of higher concentrations of plant stanols for a longer time period would 
also have elicited significant anti-inflammatory effects in the chow setting in the current study, as has also been proposed in previous discussions regarding contradicting observations of plant stanols' anti-inflammatory effects in cardiovascular disease patients (2224). Overall, although we cannot fully exclude other direct, albeit subtle, anti-inflammatory effects, our findings indicate that plant stanols' immunomodulatory effects are mainly visible in conditions of a high cholesterol intake. As such, while long-term plant stanol supplementation may benefit individuals with low-grade inflammation who do not consume excessive cholesterol, it is likely that plant stanols will bring the most benefits to patients with cholesterol-enriched, unhealthy diets. 


\section{REFERENCES}

1. Blüher M. Obesity: global epidemiology and pathogenesis. Nature Reviews Endocrinology. 2019;15(5):288-98.

2. WHO. Noncommunicable diseases: Key Facts 2018.Last updated on: 13/04/2021. Available from: https://www.who.int/news-room/fact-sheets/detail/noncommunicablediseases. Accessed on: 05/05/2021

3. Smet ED, Mensink RP, Plat J. Effects of plant sterols and stanols on intestinal cholesterol metabolism: Suggested mechanisms from past to present. Molecular Nutrition \& Food Research. 2012;56(7):1058-72.

4. Plat J, Baumgartner S, Vanmierlo T, Lütjohann D, Calkins KL, Burrin DG, et al. Plantbased sterols and stanols in health \& disease: "Consequences of human development in a plant-based environment?". Progress in Lipid Research. 2019;74:87-102.

5. Magro dos Reis I, Houben T, Oligschläger Y, Bücken L, Steinbusch H, Cassiman D, et al. Dietary plant stanol ester supplementation reduces peripheral symptoms in a mouse model of Niemann-Pick type C1 disease. Journal of Lipid Research. 2020;61(6):830-9.

6. Geberhiwot T, Moro A, Dardis A, Ramaswami U, Sirrs S, Marfa MP, et al. Consensus clinical management guidelines for Niemann-Pick disease type C. Orphanet Journal of Rare Diseases. 2018;13(1):50.

7. Bieghs V, Van Gorp PJ, Wouters K, Hendrikx T, Gijbels MJ, van Bilsen M, et al. LDL Receptor Knock-Out Mice Are a Physiological Model Particularly Vulnerable to Study the Onset of Inflammation in Non-Alcoholic Fatty Liver Disease. PLOS ONE. 2012;7(1):e30668.

8. Wouters K, Shiri-Sverdlov R, van Gorp PJ, van Bilsen M, Hofker MH. Understanding hyperlipidemia and atherosclerosis: lessons from genetically modified apoe and ldlr mice. Clinical Chemistry and Laboratory Medicine (CCLM). 2005;43(5):470-9.

9. Houben T, Oligschlaeger Y, Bitorina AV, Hendrikx T, Walenbergh SMA, Lenders M$\mathrm{H}$, et al. Blood-derived macrophages prone to accumulate lysosomal lipids trigger oxLDLdependent murine hepatic inflammation. Scientific Reports. 2017;7(1):12550.

10. Houben T, Magro dos Reis I, Oligschlaeger Y, Steinbusch H, Gijbels MJJ, Hendrikx $\mathrm{T}$, et al. Pneumococcal Immunization Reduces Neurological and Hepatic Symptoms in a Mouse Model for Niemann-Pick Type C1 Disease. Frontiers in Immunology. 2019;9:3089.

11. Bieghs V, Verheyen F, van Gorp PJ, Hendrikx T, Wouters K, Lütjohann D, et al. Internalization of Modified Lipids by CD36 and SR-A Leads to Hepatic Inflammation and Lysosomal Cholesterol Storage in Kupffer Cells. PLOS ONE. 2012;7(3):e34378.

12. Bieghs V, Wouters K, van Gorp PJ, Gijbels MJJ, de Winther MPJ, Binder CJ, et al. Role of Scavenger Receptor A and CD36 in Diet-Induced Nonalcoholic Steatohepatitis in Hyperlipidemic Mice. Gastroenterology. 2010;138(7):2477-86.e3.

13. Bieghs V, van Gorp PJ, Walenbergh SMA, Gijbels MJ, Verheyen F, Buurman WA, et al. Specific immunization strategies against oxidized low-density lipoprotein: A novel way to reduce nonalcoholic steatohepatitis in mice. Hepatology. 2012;56(3):894-903.

14. Šošić-Jurjević B, Lütjohann D, Renko K, Filipović B, Radulović N, Ajdžanović V, et al. The isoflavones genistein and daidzein increase hepatic concentration of thyroid hormones and affect cholesterol metabolism in middle-aged male rats. The Journal of Steroid Biochemistry and Molecular Biology. 2019;190:1-10. 
15. Ostlund RE, McGill JB, Zeng C-M, Covey DF, Stearns J, Stenson WF, et al. Gastrointestinal absorption and plasma kinetics of soy $\Delta 5$-phytosterols and phytostanols in humans. American Journal of Physiology-Endocrinology and Metabolism. 2002;282(4):E911E6.

16. Plat J, Hendrikx T, Bieghs V, Jeurissen MLJ, Walenbergh SMA, van Gorp PJ, et al. Protective role of plant sterol and stanol esters in liver inflammation: insights from mice and humans. PloS one. 2014;9(10):e110758-e.

17. Muse ED, Yu S, Edillor CR, Tao J, Spann NJ, Troutman TD, et al. Cell-specific discrimination of desmosterol and desmosterol mimetics confers selective regulation of LXR and SREBP in macrophages. Proceedings of the National Academy of Sciences. 2018;115(20):E4680.

18. Spann NJ, Garmire LX, McDonald JG, Myers DS, Milne SB, Shibata N, et al. Regulated accumulation of desmosterol integrates macrophage lipid metabolism and inflammatory responses. Cell. 2012;151(1):138-52.

19. Brüll F, De Smet E, Mensink RP, Vreugdenhil A, Kerksiek A, Lütjohann D, et al. Dietary plant stanol ester consumption improves immune function in asthma patients: results of a randomized, double-blind clinical trial1. The American Journal of Clinical Nutrition. 2016;103(2):444-53.

20. Brüll F, Mensink RP, Steinbusch MF, Husche C, Lütjohann D, Wesseling G-J, et al. Beneficial Effects of Sitostanol on the Attenuated Immune Function in Asthma Patients: Results of an In Vitro Approach. PLOS ONE. 2012;7(10):e46895.

21. Brüll F, Mensink RP, van den Hurk K, Duijvestijn A, Plat J. TLR2 activation is essential to induce a Th1 shift in human peripheral blood mononuclear cells by plant stanols and plant sterols. The Journal of biological chemistry. 2010;285(5):2951-8.

22. Köhler J, Teupser D, Elsässer A, Weingärtner O. Plant sterol enriched functional food and atherosclerosis. Br J Pharmacol. 2017;174(11):1281-9.

23. Cabral CE, Klein MRST. Phytosterols in the Treatment of Hypercholesterolemia and Prevention of Cardiovascular Diseases. Arq Bras Cardiol. 2017;109(5):475-82.

24. Rosin S, Ojansivu I, Kopu A, Keto-Tokoi M, Gylling H. Optimal Use of Plant Stanol Ester in the Management of Hypercholesterolemia. Cholesterol. 2015;2015:706970-. 


\section{Chapter 4}

Hematopoietic Npc1 mutation shifts gut microbiota composition in $\mathrm{Ldlr}^{--}$mice on a high-fat, high-cholesterol diet.

Tom Houben, John Penders, Yvonne Oligschlaeger, Inês Magro dos Reis, Marc-Jan Bonder, Debby P. Koonen, Jingyuan Fu, Marten H. Hofker, Ronit Shiri-Sverdlov

Sci Rep 9, 2019, 14956 


\begin{abstract}
While the link between diet-induced changes in gut microbiota and lipid metabolism in metabolic syndrome (MetS) has been established, the contribution of host genetics is rather unexplored. As several findings suggested a role for the lysosomal lipid transporter Niemann-Pick type C1 (NPC1) in macrophages during MetS, we here explored whether a hematopoietic Npc1 mutation, induced via bone marrow transplantation, influences gut microbiota composition in low-density lipoprotein receptor knockout $\left(\mathrm{Ldll}^{--}\right)$mice fed a highfat, high-cholesterol (HFC) diet for 12 weeks. $L d l r^{-/}$mice fed a HFC diet mimic a human plasma lipoprotein profile and show features of MetS, providing a model to explore the role of host genetics on gut microbiota under MetS conditions. Fecal samples were used to profile the microbial composition by 16s ribosomal RNA gene sequencing. The hematopoietic Npc1 mutation shifted the gut microbiota composition and increased microbial richness and diversity. Variations in plasma lipid levels correlated with microbial diversity and richness as well as with several bacterial genera. This study suggests that host genetic influences on lipid metabolism affect the gut microbiome under MetS conditions. Future research investigating the role of host genetics on gut microbiota might therefore lead to identification of diagnostic and therapeutic targets for MetS.
\end{abstract}




\section{BACKGROUND}

Metabolic syndrome (MetS) is a complex disorder that identifies centrally obese patients at increased risk for developing cardiovascular disease (CVD) and diabetes mellitus type 2 (1, 2). Due to the continuous increased prevalence of obesity, MetS is considered a global epidemic, putting an enormous pressure on health care services (3). Next to insulin resistance and hypertension, another central abnormality observed within MetS are disturbances in lipid metabolism, resulting in dyslipidemia $(1,4)$. Dyslipidemia is characterized by increased plasma triglyceride-rich lipoproteins, decreased plasma high-density lipoprotein (HDL) and increased plasma low-density lipoprotein (LDL), leading to MetS-associated pathologies (4).

Next to disturbances in plasma lipid levels, alterations in intracellular lipid metabolism have also been observed in MetS. While mutations in the gene encoding for the lysosomal membrane protein Niemann-Pick type C1 (NPC1) are well-known to induce a rare lysosomal storage disease, recent findings have also linked a dysfunctional NPC1 protein to the development of obesity and MetS $(5,6)$. Specifically, heterozygous Npc1 mutation carriers showed a 4.8 higher risk for developing morbid adult obesity (7). Moreover, identification of several Npc1 small nucleotide polymorphism (SNPs) via genome-wide association (5) and confirmation studies (8-10) have further highlighted the link between Npc1 variants (allele frequency $\sim 38 \%$ ) and obesity in the European population. Mechanistically, a dysfunctional NPC1 protein leads to lysosomal lipid accumulation, thereby disturbing lipid metabolism (11). Likewise, the phenomenon of lipid accumulation within the endo-lysosomal compartment of cells has also been observed in pathologies associated with MetS (12). While lysosomal dysfunction in proximal tubular cells contributed to obesity-related kidney diseases (13), lysosomal lipid storage within macrophages is a well-described mechanism disturbing general lipid metabolism and results in the induction of a chronic low-grade inflammatory response in non-alcoholic steatohepatitis $(14,15)$ and atherosclerosis $(12,16-$ 18). These findings imply a role for macrophage NPC1 in disturbing lipid homeostasis in MetS.

Relevantly, several studies have shown a link between the composition of the gut microbiota and lipid parameters such as LDL, triglyceride and HDL levels (19-23). Moreover, modulating the gut microbiota by transferring the microbiota of lean donors has been shown to improve MetS in patients (24), indicating the gut microbiota as a promising target to tackle metabolic disorders. However, the underlying mechanisms through which the gut microbiota modulates host's metabolism remain largely elusive (25). Gaining knowledge into the exact interactions between environment and host genetics on gut microbiota and associated 
pathophysiological processes is therefore essential to improve our diagnostic and therapeutic approaches to tackle metabolic pathologies.

The current study investigated whether a hematopoietic Npc1 mutation influences the gut microbiota in low-density lipoprotein receptor knockout $\left(\mathrm{Ldll} \mathrm{r}^{--}\right)$mice fed a high-fat, highcholesterol (HFC) diet for 12 weeks. Ldlr ${ }^{-2}$ mice fed a HFC diet mimic a human plasma lipoprotein profile and show features of MetS (26), providing a model to explore the role of host genetics on gut microbiota under MetS conditions. For this purpose, $L d l r^{-1}$ mice received bone marrow from Niemann-Pick type C1 mutant ( $\left.N p c 1^{m u t}\right)$ mice. This study suggests that host genetic influences related to lipid metabolism shift the gut microbiota composition and increases microbial richness and diversity under MetS conditions. Variations in plasma lipid levels correlated with microbial diversity and richness as well as with several bacterial genera. Future research investigating the role of host genetics on gut microbiota can therefore lead to identification of novel diagnostic and therapeutic targets for MetS. 


\section{RESULTS}

\section{Hematopoietic Npc1 mutation disturbs intracellular and circulating lipid levels}

To confirm successfulness of the bone marrow transplantation, intracellular and circulating lipid levels were measured in Npc1 ${ }^{m u t}$-tp $L d l r^{-/}$-mice. Subjecting $N p c 1^{m u t}$-tp $L d l r^{-/}$mice to a HFC diet for 12 weeks resulted in a strong increase in hepatic cholesterol levels, while a reduction was observed for hepatic triglyceride levels (Table 1). Moreover, due to the hematopoietic Npc1 mutation, hepatic lipids accumulated in the lysosomal fraction of hepatic macrophages (Fig. 1A - B and Supplementary Figures S1 and S2). Additionally, plasma cholesterol, triglyceride and free fatty acid levels decreased significantly, indicating a decrease of circulating lipids in $N p c 1^{m u t}$-tp $L d l r^{-/-}$mice (Table 1). In line with these data, hepatic gene expression levels of ATP-binding cassette sub-family G member 1(Abcg1), Niemann-Pick type $\mathrm{C} 2$ (Npc2) and ATP-binding cassette transporter A1 (Abca1) increased in Npc1 ${ }^{\text {mut }}$-tp L Lllr-- mice. In spite of increased hepatic cholesterol levels (Table 1), these observations indicate increased hepatic cholesterol efflux signaling, which is likely a compensation mechanism to overcome the accumulation of cholesterol in lysosomes. Overall, these data show that a hematopoietic $N p c 1$ mutation induces lysosomal accumulation of lipids inside liver macrophages and disturbs general lipid metabolism in $\mathrm{Ldlr}^{-/}$mice on a HFC diet.

A
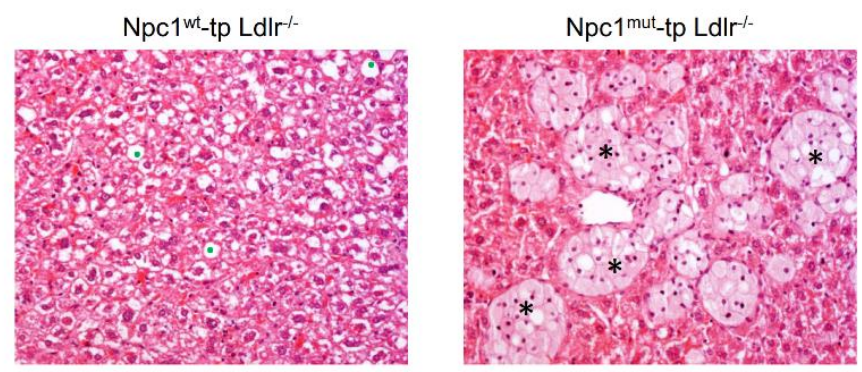

B
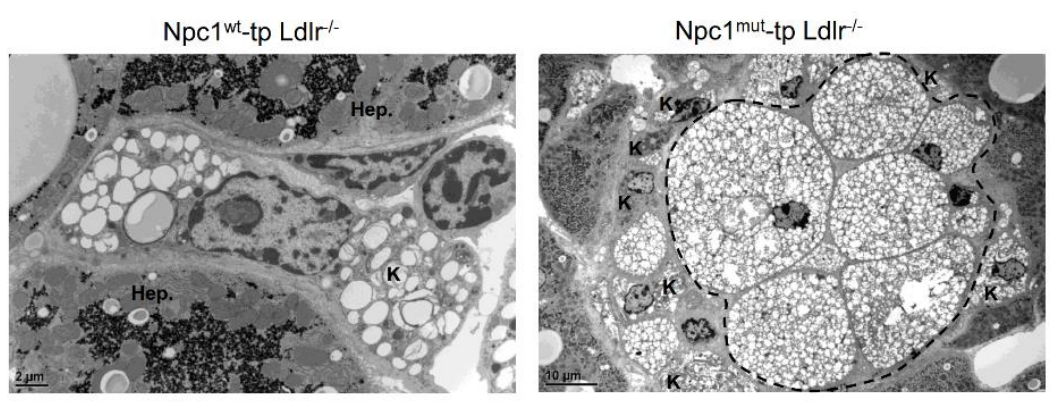
Figure 1: Hepatic phenotype of HFC-fed Ldlr-- mice carrying a hematopoietic Npc1 mutation. (A) Representative images of hepatic staining for hematoxylin and eosin (H\&E), showing lysosomal lipid accumulation and granuloma

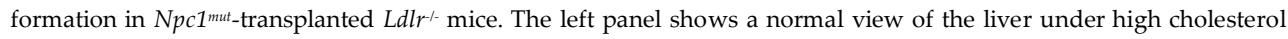
conditions, with the green dots indicating lipid droplets. The right panel show clustering of foamy macrophages, referred to as granulomas (indicated by the asterisks). (B) Representative electron microscopy image of hepatic macrophages carrying a hematopoietic Npc1 mutation in HFC-fed Ldlr- mice, confirming the massive accumulation of lipids. The left panel shows a single Kupffer cell (K), surrounded by 2 hepatocytes (Hep.). The right panel shows the granuloma (surrounded by the dashed line), which is surrounded by Kupffer cells (K).

Table 1: Intracellular and circulating lipid metabolism

\begin{tabular}{|c|c|c|}
\hline & $N p c 1^{w t_{-}} \mathbf{t p} L d l r^{-/-}$ & $N p c 1^{m u t}$-tp $L d l r^{-/-}$ \\
\hline $\begin{array}{l}\text { Liver total cholesterol } \\
(\mu \mathrm{g} / \mathrm{mg} \text { dry weight) }\end{array}$ & $47.03 \pm 4.47$ & $75.47 \pm 2.51^{* * *}$ \\
\hline $\begin{array}{l}\text { Liver total triglycerides } \\
(\mathrm{mg} / \mathrm{mg} \text { protein) }\end{array}$ & $0.51 \pm 0.06$ & $0.26 \pm 0.04^{* *}$ \\
\hline $\begin{array}{lll}\text { Plasma total cholesterol } \\
(\mathrm{mM})\end{array}$ & $38.53 \pm 1.44$ & $22.1 \pm 1.09^{* * *}$ \\
\hline $\begin{array}{l}\text { Plasma total cholesterol } \\
(\mathrm{mM})\end{array}$ & $38.53 \pm 1.44$ & $22.1 \pm 1.09^{* * *}$ \\
\hline $\begin{array}{l}\text { Plasma total triglycerides } \\
(\mathrm{mM})\end{array}$ & $3.82 \pm 0.31$ & $1.42 \pm 0.15^{* * *}$ \\
\hline Plasma free fatty acids (mM) & $1.33 \pm 0.04$ & $0.96 \pm 0.05^{* * *}$ \\
\hline Abcg1 (Rel. Expr.) & $1.00 \pm 0.09$ & $2.65 \pm 0.13^{* * *}$ \\
\hline Npc2 (Rel. Expr.) & $1.00 \pm 0.1$ & $3.06 \pm 0.12^{* * *}$ \\
\hline Abca1 (Rel. Expr.) & $1.00 \pm 0.12$ & $1.13 \pm 0.06$ \\
\hline
\end{tabular}

\section{Microbial richness and diversity are increased and linked to lipid metabolism}

To investigate the influence on microbial richness and diversity, $\alpha$-diversity metrics were calculated. Both the observed microbial richness (observed number of OTUs) as well as the estimated richness as indicated by the Chao 1 index, was significantly higher in $L d l r^{--}$mice 
carrying the hematopoietic Npc1 mutation (Fig. 2A and 2B). Likewise, microbial biodiversity, measured via the Shannon index (Fig. 2C) was significantly increased in $N p c 1^{\text {mut }}$-tp mice. To further define which variables are linked to microbial richness and diversity in this mouse model, Spearman correlation analysis was performed on all variables and the Chao 1 (richness) or Shannon (biodiversity) index. Plasma free fatty acids levels were negatively correlated with microbial richness $(\mathrm{r}=-0.47 ; \mathrm{p}=0.03$ ) (Fig. 2D). Concomitantly, plasma total cholesterol levels showed the same inverse correlation with microbial richness $(\mathrm{r}=-0.41 ; \mathrm{p}=$ 0.07) (Fig. 2E), though this correlation did not reach statistical significance. Furthermore, biodiversity correlated positively with hepatic gene expression levels of the intracellular cholesterol transporter Niemann-Pick type C2 (Npc2) $(\mathrm{r}=0.47 ; \mathrm{p}=0.04)$ (Fig. 2F) and negatively with plasma total cholesterol levels $(r=-0.56 ; p=0.007)$ (Fig. $2 \mathrm{G})$. Together, these data demonstrate that a hematopoietic $N p c 1$ mutation in $\mathrm{Ldlr}^{-/}$mice increases microbial richness and biodiversity, which is correlated to changes in lipid metabolism. 
A

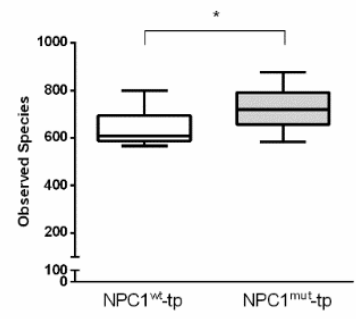

B

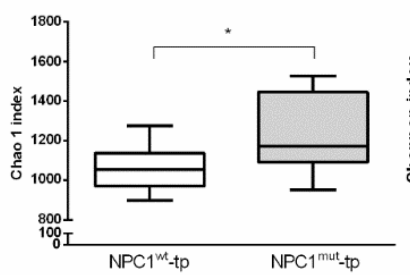

C

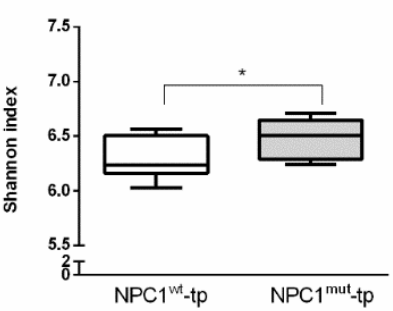

D

$\mathrm{F}$

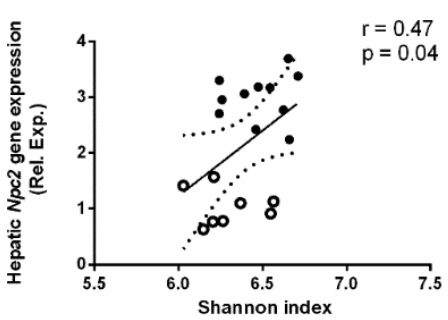

E

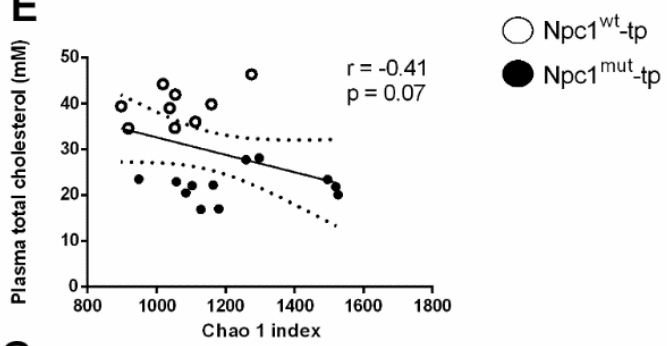

G

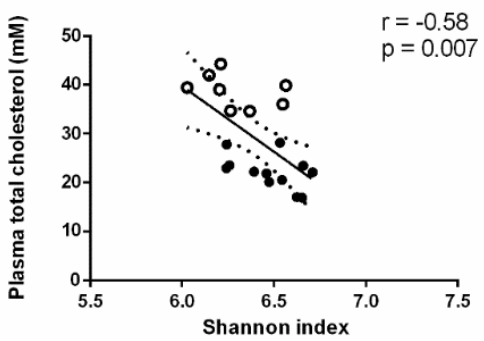

Figure 2: Alpha diversity metrics in $\mathbf{L} d l \boldsymbol{r}^{-/}$mice on HFC diet carrying a hematopoietic Npc1 mutation compared to wildtype. (A-C) The number of OTUs (A), Chao 1 index (B) and Shannon index (C) indicate increased diversity in $N p c 1^{m u t}$-transplanted $L d l r^{-/}$mice. (D-G) Spearman correlation between alpha diversity metrics and lipid parameters indicate a link between lipid metabolism and gut microbiota diversity. ${ }^{*}$ Indicates $\mathrm{p}<0.05$. All errors are SEM.

The microbial community structure is related to the hematopoietic Npc1 mutation but the latter is not linked to enterotypes

The dissimilarity in the microbial community composition (beta-diversity) of stool samples was assessed using the Bray-Curtis (BC) dissimilarities. Principal coordinate analysis (PCoA) based on Bray-Curtis dissimilarities showed that samples could be partly separated based upon the hematopoietic Npc1 mutation (Fig. 3A). Moreover, the within-group distance in microbial community structure (i.e. average Bray-Curtis dissimilarity between samples from the same treatment group) was significantly smaller than the between-group distance, indicating that the microbiota composition of animals within the same treatment group is 
more similar compared to animals from different treatment groups (Fig. 3B). Enterotype analyses revealed two enterotypes driven by Allobaculum, Lactobacillus and S24-7 (Fig. 3C). However, the clustering was not associated with the bone marrow-specific Npc1 mutation.

A

B
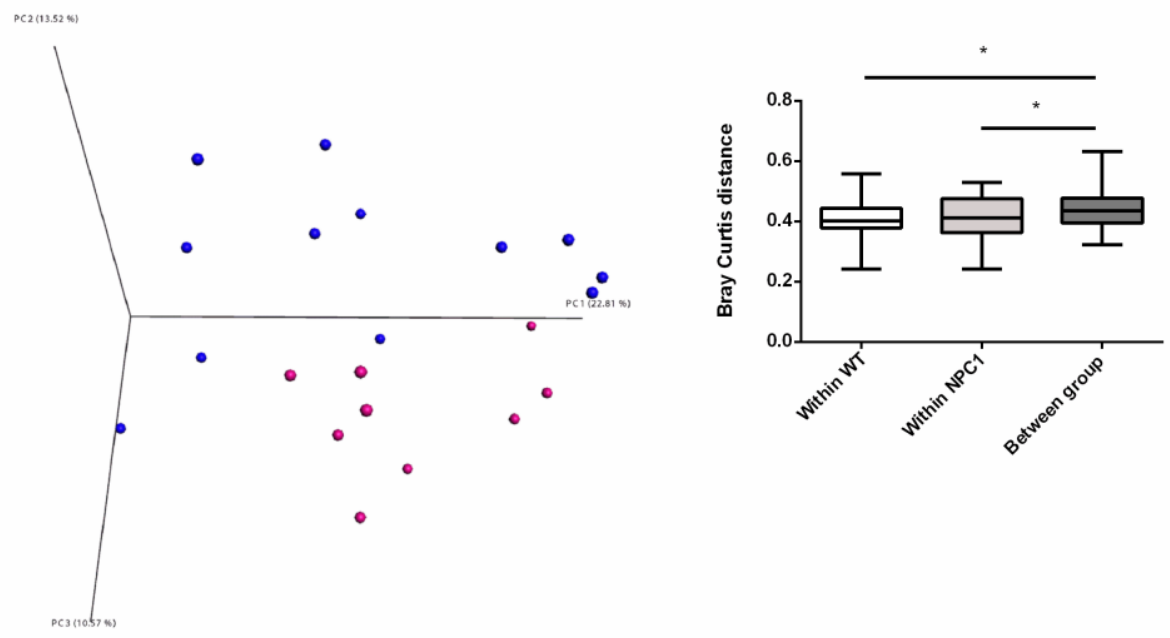

C

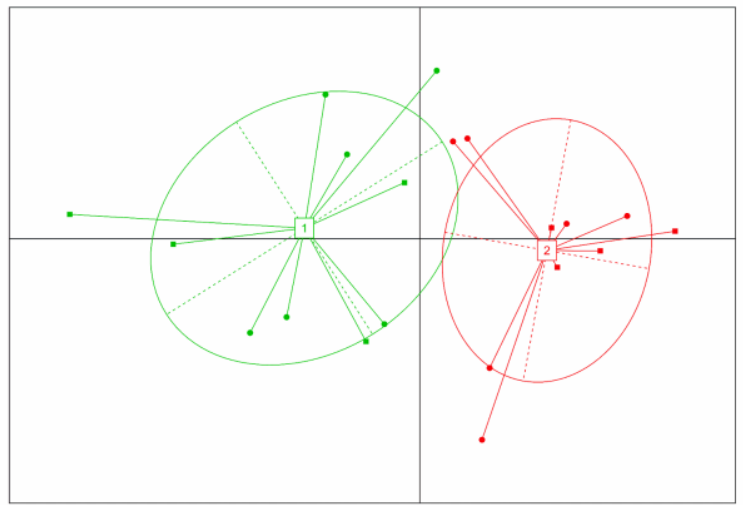

Figure 3: Principal Coordinate Analyses (PCoAs) based on Bray-Curtis distance and assortment of gut microbial communities into enterotypes for HFC-fed $L d l r^{-/}$mice carrying a hematopoietic $N p c 1^{w t}$ or Npc1 ${ }^{\text {mut }}$ mutation. (A) PCoA-plot based on unweighted UniFrac metrics for all samples showed that samples could be partly separated based upon the hematopoietic Npc1 mutation. (B) Bray-Curtis distances were calculated within Npc1 ${ }^{w t}$-transplanted and $N p c 1^{m u t}$-transplanted HFC-fed $L d l r^{-/}$mice (average pairwise distance in microbiota composition between samples of the same experimental group) and between $N p c 1^{w t}$-transplanted and $N p c 1^{\text {mut }}$-transplanted HFC-fed Ldlr- mice (average pairwise distance between samples of different experimental groups), confirming the separation based on the hematopoietic Npc1 mutation. (C) Between-class analysis visualizing the two distinct enterotypes that could be 
identified based upon PAM clustering of Bray-Curtis distances, with closed dots $\left(N p c 1^{m u t}\right)$ and squares $\left(N p c 1^{w t}\right)$ representing individual mice and numbered white rectangles marking the center of each enterotype. Enterotypes were mainly driven by differential abundances in Allobaculum, Lactobacillus and S24-7, however the clustering was not associated with the bone marrow-specific Npc1 mutation. ${ }^{*}$ Indicates $\mathrm{p}<0.05$.

To determine whether the hematopoietic Npc1 mutation or other variables had an additional effect on the gut microbiota community structure, we subsequently performed distance-based redundancy analysis (dbRDA); a constrained ordination technique. Constrained ordination techniques attempt to explain differences in microbial composition between samples by differences in explanatory variables (e.g. hematopoietic Npc1 mutation). In db-RDA, the information from explanatory variables is combined with the eigenvalues obtained from the PCoA. Permutational multivariate analysis of variance (PERMANOVA) was used to determine significant dissimilarity within bacterial communities and multiple explanatory variables. The hematopoietic Npc1 mutation ( $\mathrm{p}=0.034$, explained variance $=18.9 \%$ ) as well as hepatic gene expression levels of the cholesterol transporter Abcg1 ( $p=0.041$, explained variance $=20.8 \%$ ) showed a relationship with the microbial community structure (Fig.4A-B), whereas Abca1, Npc2, plasma total cholesterol, plasma total triglycerides, plasma free fatty acids, plasma cupper oxLDL, plasma EO6, cage number, liver total cholesterol and liver total triglycerides did not.

\section{Hematopoietic Npc1 mutation shifts gut microbiota composition in HFC-fed $\mathrm{Ldlr}^{-/-}$mice}

Based on 16S rRNA gene sequencing, the dominant phyla/genera were selected for further analysis, leading to in depth analysis of 4 phyla and 44 genera (Fig. 5A-B). Firmicutes, Bacteroidetes and, to a lesser extent, Actinobacteria and Proteobacteria were the dominant observed phyla (Fig. 5A). The relative abundance of the bacterial phyla was not significantly different between $N p c 1^{\text {wt }}$ - and $N p c 1^{m u t}$-tp mice (Fig. 5A), however, within the Firmicutes phylum significant differences were observed in the relative abundance of several bacterial genera. Specifically, Staphylococcus spp. $(\mathrm{p}=0.008 ; \mathrm{q}=0.34)$ and unclassified Mogibacteriaceae spp. $(\mathrm{p}=0.008 ; \mathrm{q}=0.34)$ showed significantly higher levels in relative abundance in the $N p c 1^{m u t}$-tp mice compared to Npc1wt-tp mice, whereas the abundance of Allobaculum spp. ( $\mathrm{p}=$ 0.01, q = 0.34) was significantly lower (Fig. 5B-E). Relevantly, the unclassified Mogibacteriaceae spp. correlated with hepatic gene expression levels of $A b c g 1(\mathrm{r}=0.57 ; \mathrm{p}=0.009)($ Fig. 5F) and plasma total triglyceride levels $(\mathrm{r}=-0.41 ; \mathrm{p}=0.07)$, though the latter association was not statistically significant (Fig. 5G). Furthermore, the genus Allobaculum spp. showed a positive correlation with plasma free fatty acid levels $(r=0.52 ; p=0.02)$ (Fig. $5 \mathrm{H})$. Thus, a hematopoietic Npc1 mutation alters the composition of gut microbiota in HFC-fed Ldlr-- mice, which are correlated with changes in lipid metabolism. 
A

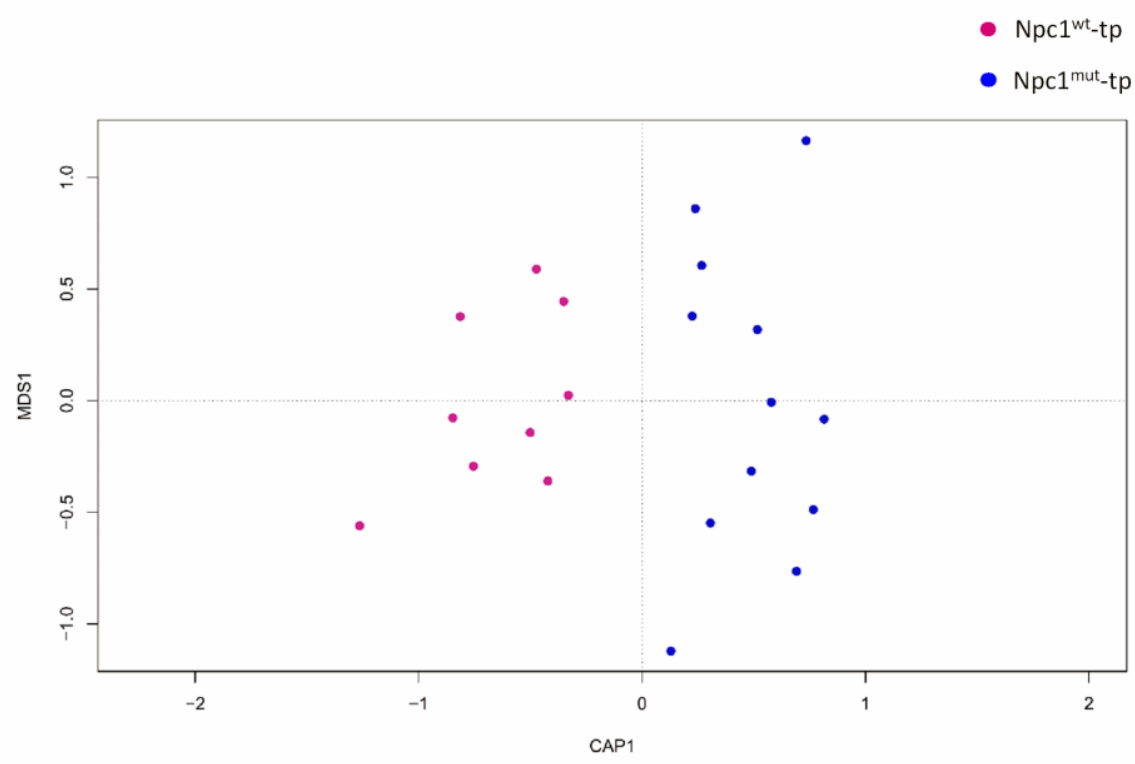

B

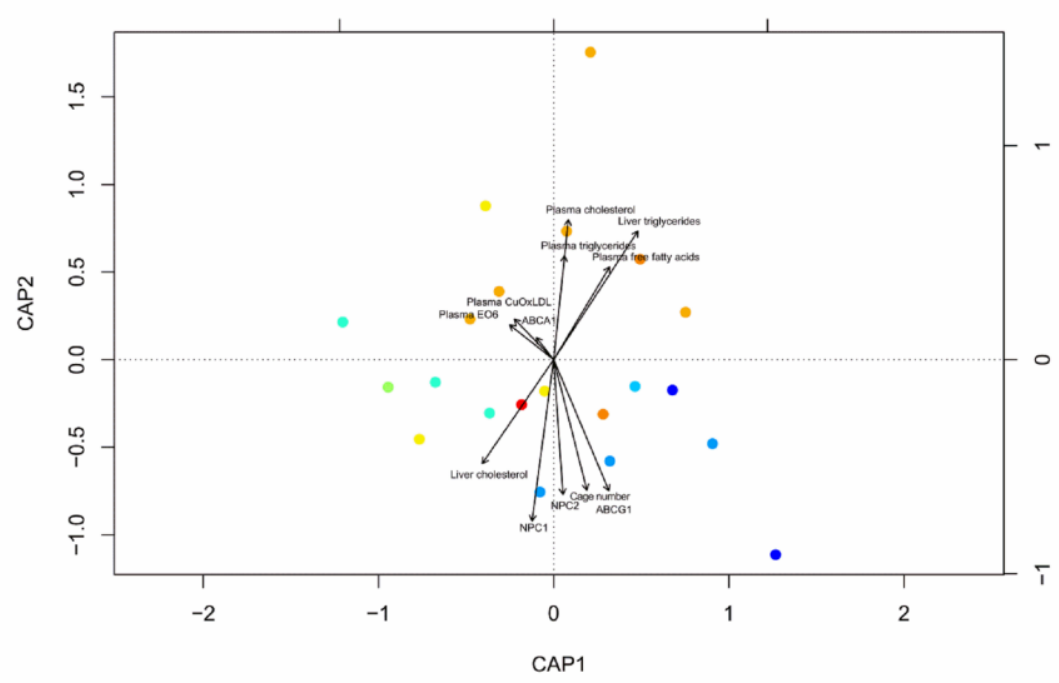

Low

High 
Figure 4: Distance-based Redundancy Analysis (db-RDA) showing the relationship of the hematopoietic deficiency for Npc1 and Abcg1 to the microbial community structure. The plots represent a dbRDA ordination based upon the Bray-Curtis distance including (A) $N p c 1^{w t}$ - and $N p c 1^{\text {mut }}$-transplanted Ldlr/- mice with the hematopoietic Npc1 mutation as explanatory variable. This panel confirms the separation of the microbiota based on the hematopoietic Npc1 mutation. (B) Plasma cholesterol, plasma triglycerides, liver triglycerides, plasma free fatty acids, cage number, liver cholesterol, plasma EO6, plasma CuOxLDL and hepatic gene expression of $A b c g 1, N p c 2, N p c 1$ and $A b c a 1$ as explanatory variables and samples colored to hepatic expression of $A b c g 1$. Multivariate PERMANOVA analysis including all explanatory variables demonstrated that both $N p c 1$ and $A b c g 1$ were significantly associated with the microbial community structure, while all other explanatory variables were not when mutually adjusting for each other. This plot further confirms that increased $\mathrm{Npc1}$ and $\mathrm{Abcg1}$ expression levels are associated with similar changes in the microbial community structure. MDS1 represents the unconstrained axis. CAP1 and CAP2 represent, respectively, the first and second constrained axes used in the CAP (canonical analysis of principal coordinates). 
A

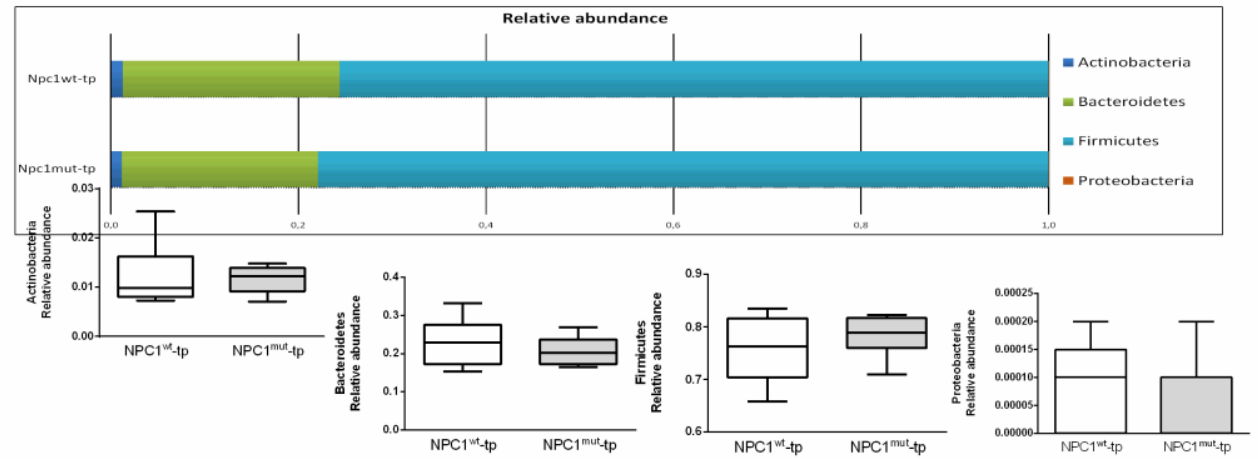

B

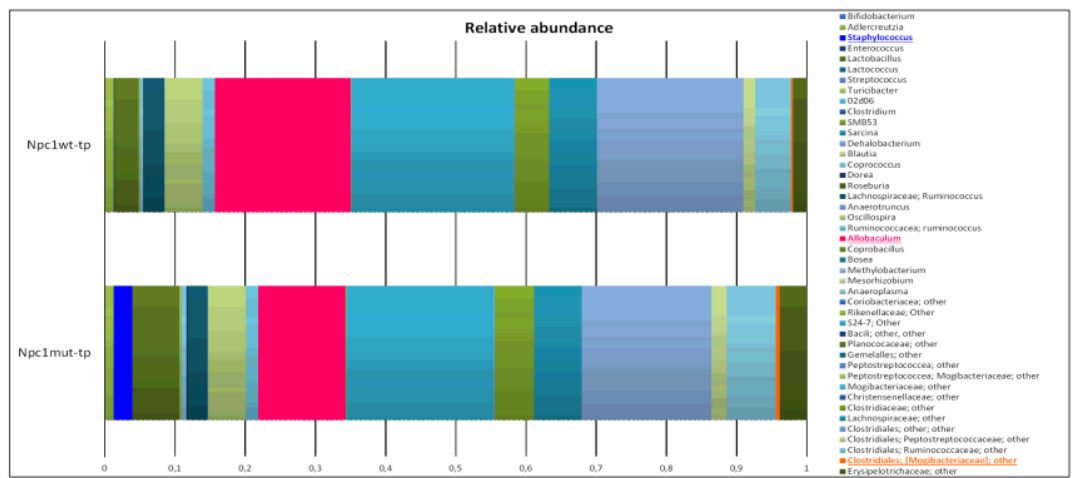

C

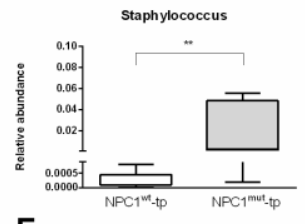

$\mathbf{F}$

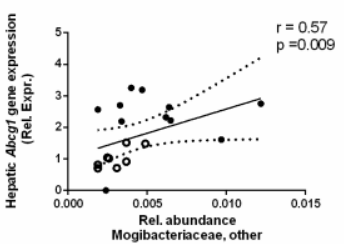

D

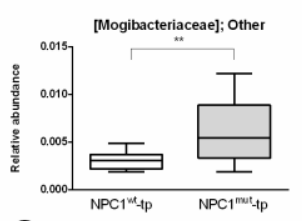

G

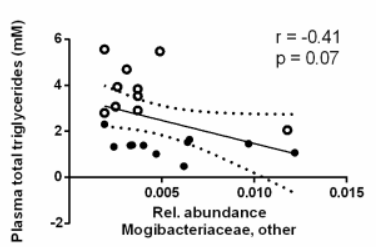

E

Allobaculum

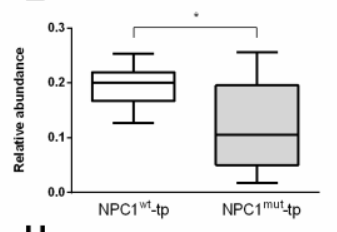

H

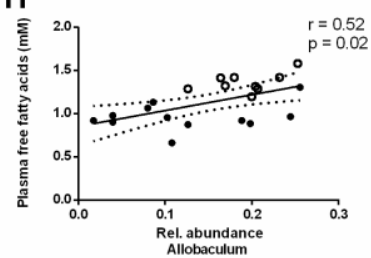

Figure 5: Taxonomy at phylum and genus level for $N p c 1^{w t}$ - and $N p c 1^{\text {mut }}$-transplanted HFC-fed Ldlr'- mice. (A-B) Overview of the mean relative abundance at phylum level. (C) An overview of the mean relative abundance at genus level is given. Significant differences between the groups at genus level are presented in separate box-whiskers for Staphylococcus spp. (D), unclassified Mogibacteriaceae spp. (E) and Allobaculum spp. (F). (G-I) Spearman correlation between lipid parameters and relative abundances of unclassified Mogibacteriaceae spp. and Allobaculum spp. ${ }^{*}$ Indicates $\mathrm{p}<0.05$ and ${ }^{* *} \mathrm{p}<0.01$. 


\section{DISCUSSION}

Whereas increasing evidence has linked diet-induced changes in gut microbiota to perturbations in lipid metabolism in MetS, the contribution of host genetics to this relation is rather unexplored. This study suggests that host genetic influences on lipid metabolism such as a hematopoietic Npc1 mutation affect the gut microbiome under MetS conditions.

Though Npc1 was previously identified in a human GWAS for obesity (5), our study is the first to imply its direct impact on the gut microbiome under MetS conditions. This observation suggests that host-derived genetic disturbances in lipid metabolism can impact the gut microbiome. Indeed, an increasing amount of reports have established the capacity of the host's genetic profile to shape the gut microbiome, thereby supporting our findings $(27,28)$. Also, the observation that a hematopoietic Npc1 mutation impacts the gut microbiota under MetS conditions confirms the previously established link between lipid metabolism and gut microbiota composition. However, in contrast to genetic manipulation of lipid metabolism, the link between lipid metabolism and gut microbiota has mainly been established based on studies wherein dietary components (i.e. high fat diet, prebiotics) altered lipid metabolism via influences on gut microbiota $(29,30)$. Therefore, in addition to gut microbiota-mediated influences of dietary compounds on lipid metabolism, our study implies that also host genetic factors that influence lipid metabolism can impact the gut microbiome. However, as we employed a HFC diet to both experimental conditions, the observed impact of the gut microbiota of the Npc1 mutation is likely also influenced by this diet. Indeed, previous reports have indicated that a high fat diet influences the composition of the gut microbiota $(31,32)$. Therefore, in spite of the observed changes in gut microbiota composition, to incontrovertibly demonstrate that the hematopoietic Npc1 mutation influences gut microbiota composition, it is essential to investigate the gut microbiota of $N p c 1^{m u t}-t p L d l r^{-1-}$ mice receiving a normal chow diet.

Our results also shed new light on the association between overall health status and the gut bacterial richness and diversity. Here, under HFC conditions, induction of the pathological hematopoietic Npc1 mutation elevated bacterial richness and diversity. However, this is opposed to the concept that increased bacterial richness and diversity reflect ecosystem stability and resilience and is associated with overall health (33-37). Yet, our apparent discordant finding on the association between disease and richness/diversity has also been observed by Kasai et al. who observed increased levels of bacterial diversity in obese compared to lean subjects (38). Moreover, Vandeputte et al. associated stool consistency, potentially due to long colonic transit time $(39,40)$, with higher gut bacterial richness. Therefore, the increased bacterial richness and diversity observed in the pathological 
experimental group in the current study might have been influenced by stool consistency, hampering the view on bacterial richness and diversity as a marker for overall health status. Of note, while it is generally accepted that reduced plasma lipids are beneficial for health (41), in the current model the reduced plasma lipids are a consequence of the accumulation of lipids inside macrophages (due to the Npc1 mutation). Therefore, the observed reduction in plasma lipids in our model is not considered beneficial for the health status, as these mice develop inflammation in the liver (42) and arteries (43).

Among the shifted gut bacteria after hematopoietic Npc1 mutation, we identified reduced relative abundances of the genus Allobaculum spp.. Several studies have previously shown that Allobaculum spp. abundance is reduced in response to dietary fat intake $(44,45)$ and correlates with variations in plasma HDL concentrations (46) and body weight (47). In line, hepatic Abcg1 and Abca1 expression was increased in Npc1 ${ }^{m u t}$-tp mice, suggesting changes in lipid metabolism via lysosomal lipid-mediated activation of the transcription factor liver $\mathrm{X}$ receptor $(48,49)$. Therefore, our finding is consistent with the proposed view that Allobaculum spp. can be considered beneficial for the physiology of the host (29). Next, in a prospective cross-sectional study, Staphylococcus spp. abundance was positively correlated with energy intake in obese children (50). Here, we found an increased abundance of Staphylococcus spp. in HFC-fed $L d l r^{--}$mice carrying the hematopoietic Npc1 mutation. As such, Staphylococcus spp. might be involved in regulating the host's lipid metabolism. Finally, increased relative abundances were observed of unclassified Mogibacteriaceae spp. in this study. Relevantly, a recent study by Harach et al. correlated levels of unclassified Mogibacteriaceae spp. with amyloid beta 42 levels in the brain of mice suffering from Alzheimer's disease (51). As multiple reports have previously linked a dysfunctional NPC1 protein to Alzheimer's disease (52-54), relative abundance changes in unclassified Mogibacteriaceae spp. might be directly related to mutations in $N p c 1$ and deserve further investigation. Of note, to ensure the causality between mutations in Npc1 and changes in gut microbiota composition, host responses should also be investigated in the future by means of fecal transplantation experiments. As such, the results in the current study do not provide information on causality, but are rather of associative nature.

Overall, this study suggests the impact of host genetic influences on lipid metabolism such as a hematopoietic Npc1 mutation on the gut microbiome in the context of MetS, confirming the link between lipid metabolism and gut microbiota. In addition, in contrast to previous studies, our findings suggest that increased microbial richness and diversity do not necessarily associate with improved health. Despite the significant associations between microbial richness and diversity and several lipid parameters, these Spearman correlations were rather low, potentially due to low sample size. As such, further research is necessary to 
confirm these associations. Finally, future research investigating the role of host genetics on gut microbiota is therefore warranted as it will increase our understanding of the function of the gut microbiome in human physiology and pathology. 


\section{METHODS}

\section{Experimental design}

Niemann-Pick type $\mathrm{C} 1^{\text {nih }}$ mutant $\left(N p c 1^{m u t}\right)$ mice (a kind gift from Prof. Dr. Lieberman from University of Michigan Medical School) were backcrossed into a C57BL/6J background for more than 10 generations. $N p c 1^{m u t}$ and $L d l r^{--}$mice were housed under standard conditions and had access to food and water ad libitum. Experiments were performed according to Dutch regulations and approved by the Committee for Animal Welfare of Maastricht University (approval number DEC 2011-133) (42,43). Specifically, mice were housed in individually ventilated cages (IVC) in a conventional specified pathogenic free (SPF) facility where a $12 \mathrm{hr}$ day/night cycle was handled using a dusk transition phase. Room temperature was kept constant at $21^{\circ} \mathrm{C}$. Corncob bedding material was used in the cages and animals were fed $10 \mathrm{~mm}$ food pellets that were irradiated with $25 \mathrm{kGray}$.

To generate myeloid $N p c 1^{m u t}$ deficient $L d l r^{-/}$mice, a bone marrow transplantation was performed. Twenty-two week-old female $\mathrm{Ldlr}^{-}$mice received one week before and four weeks after irradiation antibiotic water containing $100 \mathrm{mg} / \mathrm{l}$ neomycin (Gibco, Breda, the Netherlands) and $6^{*} 10^{4} \mathrm{U} / 1$ polymycin B Sulphate (Gibco, Breda, the Netherlands). One day before and on the day of the transplantation, $L d l r^{-1-}$ mice were lethally irradiated with 6 Gray of $\gamma$-radiation in the morning, thus receiving 12 Gray in total. Lethally irradiated $\mathrm{Ldlr}{ }^{-1}$ mice were then injected with $1^{*} 10^{7}$ bone marrow cells donated from either $N p c 1^{\text {mut }}$ mice or wildtype littermate controls $\left(N p c 1^{w t}\right)$. In order to fully ensure bone marrow replacement, mice had a nine-week recovery period. After nine weeks of recovery, transplanted (-tp) mice received an HFC diet, containing $21 \%$ butter, $0.2 \%$ cholesterol, $46 \%$ carbohydrates and $17 \%$ casein (diet 1635; Scientific Animal Food and Engineering, Villemoissonsur-Orge, France) for 12 weeks. We further highlight that the same mouse model as well as the described lipid measurements were used in previously published manuscripts, where administration of dietary cholesterol was essential to induce lysosomal lipid-induced inflammation $(42,43,55)$. As we anticipated only subtle changes (56) in the gut microbiome due to the Npc1 mutation being restricted to the hematopoietic line, we did not cohouse both genotypes. Though the influence of cageeffects cannot be completely excluded, the following measures were taken to minimize the impact of housing conditions: the litters were separated per genotype after weaning, mice of similar age were used, the entire experiment was performed at once (so not over different time points), food and autoclaving parameters were standardized, only 1 person handled the mice throughout the entire experiment, mice were kept in cages in the same room, in the same rack and finally, mice of each genotype were put in four different cages. Finally, the betweengenotype Bray-Curtis dissimilarity was significantly increased to the between-cage BrayCurtis dissimilarity ( $\left(p=0.03\right.$ compared to WT cages and $\mathrm{p}=0.04$ compared to Npc1 ${ }^{\text {mut }}$ cages), providing further evidence that the observed effects in the current study are at least partly 
due to the $N p c 1$ mutation. Liver tissue was isolated and snap-frozen in liquid nitrogen and stored at $-80^{\circ} \mathrm{C}$ or fixed in $4 \%$ formaldehyde/PBS. The biochemical determination of plasma cholesterol and liver triglyceride levels, electron microscopy, RNA isolation, complementary DNA synthesis, quantitative polymerase chain reaction are described extensively $(42,43,57)$. Liver cholesterol levels were quantified as described previously(58).

\section{Sample collection and processing}

Mice were housed in groups of 4 in separate cages, which were kept in the same room throughout the study. Fecal samples were snap-frozen with liquid nitrogen and stored at $80^{\circ} \mathrm{C}$. DNA isolation was done using the FavorPrep Stool DNA Isolation Mini Kit (FASTI 0011) according to manufacturer's protocol.

\section{Sequencing}

Amplicon libraries and sequencing was performed according to previously published protocols $(19,59,60)$. Briefly, the hypervariable region V4 of the $16 \mathrm{~S}$ rRNA gene was PCR amplified from each DNA sample using forward primer 515F and reverse primer $806 \mathrm{R}$ as described previously (19). Illumina MiSeq paired end sequencing of equimorlarly pooled amplicons was subsequently used to determine the bacterial composition of fecal samples. Custom scripts were used to remove primer sequences, align paired end reads and qualityfilter the sequencing reads. Details can be found in $\mathrm{Fu}$ et al. (19) and Bonder et al. (59). Sequencing data of all samples have been deposited in the European Bioinformatics Institute (EBI) database under primary accession code (ENA) PRJEB31646 and secondary accession code ERP114028.

\section{Data analysis and statistics}

Sequences were clustered into Operational Taxonomic Units (OTUs) using UCLUST (version 1.2.22q) at 97\% similarity. For taxonomic assignments of OTUs, a TaxMan (61) filtered and truncated version of the full Greengenes reference database version 13.5 was used. OTUs that did not cluster against any of the sequences in the reference database were suppressed.

A total of 1,503,804 paired-end sequences, with a sequencing depth ranging from 15,637117,585 reads/sample, were clustered in a total of 13,312 OTUs. To further reduce spurious OTUs and normalize for sequencing depth, data were rarefied to 10,000 reads/sample and OTUs containing less than 5 reads or occurring in only a single sample were discarded, resulting in a final number of 2.695 OTUs. Downstream analysis were conducted in QIIME version 1.9 (62) and R version 3.1.3.

\section{Microbial data-analyses and statistics}

Taxonomic composition 
Differences in the relative abundance of bacterial phyla and genera between $N p c 1^{w t}$-tp and $N p c 1^{m u t}$-tp $L d l r^{-1}$ mice were compared using the non-parametric t-test Metastats while controlling for multiple comparisons by means of the False Discovery Rate $(q=0.15 ; 750$ permutations). Relations between bacterial phyla/genera and other continuous variables were analyzed via Spearman correlation using GraphPad Prism, version 6.0 for Windows. Though correlations between lipid parameters and gut microbiota abundances were calculated on pooled data to increase the power, it was verified whether both groups showed the same effect.

\section{Microbial richness $\mathcal{E}$ diversity and microbial community structure}

The following metrics of species richness and diversity within communities (alpha-diversity) were determined: observed OTUs (observed richness), Chao1 index (estimated richness), and Shannon diversity index. Alpha diversity metrics between the wild-type and mutant animals were compared by Mann Whitney tests using GraphPad Prism, version 6.0 for Windows. Beta-diversity, or diversity shared across samples was determined by the Bray-Curtis dissimilarity (BC) at a rarefaction depth of 10,000 seq/sample (Supplementary Figure S3). Clustering of samples was visualized using Principal Coordinate analysis (PCoA) followed by distance-based redundancy analysis (db-RDA), a constrained extension of PCoA. Db-RDA was performed in $\mathrm{R}$ (63) package vegan using the capscale function. To determine to what extend the hematopoietic Npc1 mutation contributed in explaining the microbial community structure, we used variance partitioning with distance-based redundancy analysis (db-RDA) of Bray-Curtis distances. In a subsequent db-RDA, the following data were additionally included as explanatory variables to examine whether they had a significant impact on the microbial community structure: liver cholesterol, liver triglycerides, plasma cholesterol, plasma triglycerides, plasma free fatty acids, plasma cupper oxLDL, plasma EO6, cage number and hepatic gene expression of Abcg1, Npc2 and Abca1.

\section{Enterotyping analyses}

Enterotype analyses were performed as described previously by Armougham and colleagues (64). Bray-Curtis (BC) distances were calculated for the genus-level relative abundance profiles. We used the R (63) package "vegan: Community ecology", version 2.2-1 by Oksanen et al. from 2011 for calculating the Bray-Curtis distances (65). To cluster the samples based on these distance metrics, we used the Partitioning Around Medoids (PAM) clustering algorithm in the R package "Cluster analysis basics and extensions", version 2.0.1 ed. by Maechler et al. from 2012 (66). The optimal number of clusters was chosen based on Calinski-Harabasz $(\mathrm{CH})$ index and validated by the silhouette index (67) using the R package 'clusterSim'. An optimal number of 2 clusters was identified based on the $\mathrm{CH}$ index, which was confirmed by the silhouette index although clustering was weak (SI 0.28). Subsequent visualization and 
identification of relevant taxa was conducted for analyses based on BC distance. Betweenclass analysis (BCA) was performed to plot the samples using the R package "Analysis of Ecological Data: Exploratory and Euclidean Methods in Environmental Sciences" version 1.7.2. by Dray et al. from 2015. The similarity percentage analysis (SIMPER) (68) was used to identify taxa contributing to similarity within- and dissimilarity between groups. Similar analyses have also been described previously (60). 


\section{REFERENCES}

1. Kassi E, Pervanidou P, Kaltsas G, Chrousos G. Metabolic syndrome: definitions and controversies. BMC Med. 2011;9:48.

2. Hotamisligil GS. Inflammation and metabolic disorders. Nature. 2006;444(7121):860-

7.

3. Boudreau DM, Malone DC, Raebel MA, Fishman PA, Nichols GA, Feldstein AC, et al. Health care utilization and costs by metabolic syndrome risk factors. Metab Syndr Relat Disord. 2009;7(4):305-14.

4. Ruotolo G, Howard BV. Dyslipidemia of the metabolic syndrome. Curr Cardiol Rep. 2002;4(6):494-500.

5. Meyre D, Delplanque J, Chevre JC, Lecoeur C, Lobbens S, Gallina S, et al. Genomewide association study for early-onset and morbid adult obesity identifies three new risk loci in European populations. Nat Genet. 2009;41(2):157-9.

6. Jelinek D, Castillo JJ, Heidenreich RA, Garver WS. The C57BL/6J Niemann-Pick C1 mouse model with decreased gene dosage is susceptible to increased weight gain when fed a high-fat diet: Confirmation of a gene-diet interaction. Gene. 2015;568(1):112-3.

7. Liu R, Zou Y, Hong J, Cao M, Cui B, Zhang H, et al. Rare Loss-of-Function Variants in NPC1 Predispose to Human Obesity. Diabetes. 2017;66(4):935-47.

8. Sandholt CH, Vestmar MA, Bille DS, Borglykke A, Almind K, Hansen L, et al. Studies of metabolic phenotypic correlates of 15 obesity associated gene variants. PLoS One. 2011;6(9):e23531.

9. Cotsapas C, Speliotes EK, Hatoum IJ, Greenawalt DM, Dobrin R, Lum PY, et al. Common body mass index-associated variants confer risk of extreme obesity. Hum Mol Genet. 2009;18(18):3502-7.

10. Berndt SI, Gustafsson S, Magi R, Ganna A, Wheeler E, Feitosa MF, et al. Genomewide meta-analysis identifies 11 new loci for anthropometric traits and provides insights into genetic architecture. Nat Genet. 2013;45(5):501-12.

11. Miller WL, Bose HS. Early steps in steroidogenesis: intracellular cholesterol trafficking. J Lipid Res. 2011;52(12):2111-35.

12. Hendrikx T, Walenbergh SM, Hofker MH, Shiri-Sverdlov R. Lysosomal cholesterol accumulation: driver on the road to inflammation during atherosclerosis and non-alcoholic steatohepatitis. Obes Rev. 2014;15(5):424-33.

13. Yamamoto T, Takabatake Y, Takahashi A, Kimura T, Namba T, Matsuda J, et al. High-Fat Diet-Induced Lysosomal Dysfunction and Impaired Autophagic Flux Contribute to Lipotoxicity in the Kidney. J Am Soc Nephrol. 2016.

14. Bieghs V, van Gorp PJ, Walenbergh SM, Gijbels MJ, Verheyen F, Buurman WA, et al. Specific immunization strategies against oxidized low-density lipoprotein: a novel way to reduce nonalcoholic steatohepatitis in mice. Hepatology. 2012;56(3):894-903.

15. Ioannou GN. The Role of Cholesterol in the Pathogenesis of NASH. Trends Endocrinol Metab. 2016;27(2):84-95.

16. Settembre C, Ballabio A. Lysosome: regulator of lipid degradation pathways. Trends Cell Biol. 2014;24(12):743-50.

17. Weber K, Schilling JD. Lysosomes integrate metabolic-inflammatory cross-talk in primary macrophage inflammasome activation. J Biol Chem. 2014;289(13):9158-71. 
18. Xu X, Yuan X, Li N, Dewey WL, Li PL, Zhang F. Lysosomal cholesterol accumulation in macrophages leading to coronary atherosclerosis in CD38(-/-) mice. J Cell Mol Med. 2016;20(6):1001-13.

19. Fu J, Bonder MJ, Cenit MC, Tigchelaar EF, Maatman A, Dekens JA, et al. The Gut Microbiome Contributes to a Substantial Proportion of the Variation in Blood Lipids. Circ Res. 2015;117(9):817-24.

20. Caesar R, Nygren H, Oresic M, Backhed F. Interaction between dietary lipids and gut microbiota regulates hepatic cholesterol metabolism. J Lipid Res. 2016;57(3):474-81.

21. Caesar R, Tremaroli V, Kovatcheva-Datchary P, Cani PD, Backhed F. Crosstalk between Gut Microbiota and Dietary Lipids Aggravates WAT Inflammation through TLR Signaling. Cell Metab. 2015;22(4):658-68.

22. Zhong CY, Sun WW, Ma Y, Zhu H, Yang P, Wei H, et al. Microbiota prevents cholesterol loss from the body by regulating host gene expression in mice. Sci Rep. 2015;5:10512.

23. Zhao L. The gut microbiota and obesity: from correlation to causality. Nat Rev Microbiol. 2013;11(9):639-47.

24. Vrieze A, Van Nood E, Holleman F, Salojarvi J, Kootte RS, Bartelsman JF, et al. Transfer of intestinal microbiota from lean donors increases insulin sensitivity in individuals with metabolic syndrome. Gastroenterology. 2012;143(4):913-6 e7.

25. Sommer F, Backhed F. The gut microbiota--masters of host development and physiology. Nat Rev Microbiol. 2013;11(4):227-38.

26. Ishibashi S, Brown MS, Goldstein JL, Gerard RD, Hammer RE, Herz J. Hypercholesterolemia in low density lipoprotein receptor knockout mice and its reversal by adenovirus-mediated gene delivery. J Clin Invest. 1993;92(2):883-93.

27. Goodrich JK, Waters JL, Poole AC, Sutter JL, Koren O, Blekhman R, et al. Human genetics shape the gut microbiome. Cell. 2014;159(4):789-99.

28. Bonder MJ, Kurilshikov A, Tigchelaar EF, Mujagic Z, Imhann F, Vila AV, et al. The effect of host genetics on the gut microbiome. Nat Genet. 2016;48(11):1407-12.

29. Everard A, Lazarevic V, Gaia N, Johansson M, Stahlman M, Backhed F, et al. Microbiome of prebiotic-treated mice reveals novel targets involved in host response during obesity. ISME J. 2014;8(10):2116-30.

30. Turnbaugh PJ, Backhed F, Fulton L, Gordon JI. Diet-induced obesity is linked to marked but reversible alterations in the mouse distal gut microbiome. Cell Host Microbe. 2008;3(4):213-23.

31. He C, Cheng D, Peng C, Li Y, Zhu Y, Lu N. High-Fat Diet Induces Dysbiosis of Gastric Microbiota Prior to Gut Microbiota in Association With Metabolic Disorders in Mice. Front Microbiol. 2018;9:639.

32. Brandsma E, Kloosterhuis NJ, Koster M, Dekker DC, Gijbels MJJ, van der Velden S, et al. A Proinflammatory Gut Microbiota Increases Systemic Inflammation and Accelerates Atherosclerosis. Circ Res. 2019;124(1):94-100.

33. Eckburg PB, Bik EM, Bernstein CN, Purdom E, Dethlefsen L, Sargent M, et al. Diversity of the human intestinal microbial flora. Science. 2005;308(5728):1635-8.

34. Le Chatelier E, Nielsen T, Qin J, Prifti E, Hildebrand F, Falony G, et al. Richness of human gut microbiome correlates with metabolic markers. Nature. 2013;500(7464):541-6. 
35. Lozupone CA, Stombaugh JI, Gordon JI, Jansson JK, Knight R. Diversity, stability and resilience of the human gut microbiota. Nature. 2012;489(7415):220-30.

36. Cotillard A, Kennedy SP, Kong LC, Prifti E, Pons N, Le Chatelier E, et al. Dietary intervention impact on gut microbial gene richness. Nature. 2013;500(7464):585-8.

37. Mosca A, Leclerc M, Hugot JP. Gut Microbiota Diversity and Human Diseases: Should We Reintroduce Key Predators in Our Ecosystem? Front Microbiol. 2016;7:455.

38. Kasai C, Sugimoto K, Moritani I, Tanaka J, Oya Y, Inoue H, et al. Comparison of the gut microbiota composition between obese and non-obese individuals in a Japanese population, as analyzed by terminal restriction fragment length polymorphism and nextgeneration sequencing. BMC Gastroenterol. 2015;15:100.

39. Vandeputte D, Falony G, Vieira-Silva S, Tito RY, Joossens M, Raes J. Stool consistency is strongly associated with gut microbiota richness and composition, enterotypes and bacterial growth rates. Gut. 2016;65(1):57-62.

40. Roager HM, Hansen LB, Bahl MI, Frandsen HL, Carvalho V, Gobel RJ, et al. Colonic transit time is related to bacterial metabolism and mucosal turnover in the gut. Nat Microbiol. 2016;1(9):16093.

41. Hardman AE. Physical activity, obesity and blood lipids. Int J Obes Relat Metab Disord. 1999;23 Suppl 3:S64-71.

42. Houben T, Oligschlaeger Y, Bitorina AV, Hendrikx T, Walenbergh SMA, Lenders $\mathrm{MH}$, et al. Blood-derived macrophages prone to accumulate lysosomal lipids trigger oxLDLdependent murine hepatic inflammation. Sci Rep. 2017;7(1):12550.

43. Jeurissen MLJ, Walenbergh SMA, Houben T, Gijbels MJJ, Li J, Hendrikx T, et al. Prevention of oxLDL uptake leads to decreased atherosclerosis in hematopoietic NPC1deficient Ldlr(-/-) mice. Atherosclerosis. 2016;255:59-65.

44. Cani PD, Bibiloni R, Knauf C, Waget A, Neyrinck AM, Delzenne NM, et al. Changes in gut microbiota control metabolic endotoxemia-induced inflammation in high-fat dietinduced obesity and diabetes in mice. Diabetes. 2008;57(6):1470-81.

45. Fleissner CK, Huebel N, Abd El-Bary MM, Loh G, Klaus S, Blaut M. Absence of intestinal microbiota does not protect mice from diet-induced obesity. Br J Nutr. 2010;104(6):919-29.

46. Martinez I, Wallace G, Zhang C, Legge R, Benson AK, Carr TP, et al. Diet-induced metabolic improvements in a hamster model of hypercholesterolemia are strongly linked to alterations of the gut microbiota. Appl Environ Microbiol. 2009;75(12):4175-84.

47. Ravussin Y, Koren O, Spor A, LeDuc C, Gutman R, Stombaugh J, et al. Responses of gut microbiota to diet composition and weight loss in lean and obese mice. Obesity (Silver Spring). 2012;20(4):738-47.

48. Mony VK, Benjamin S, O'Rourke EJ. A lysosome-centered view of nutrient homeostasis. Autophagy. 2016;12(4):619-31.

49. Venkateswaran A, Laffitte BA, Joseph SB, Mak PA, Wilpitz DC, Edwards PA, et al. Control of cellular cholesterol efflux by the nuclear oxysterol receptor LXR alpha. Proc Natl Acad Sci U S A. 2000;97(22):12097-102.

50. Bervoets L, Van Hoorenbeeck K, Kortleven I, Van Noten C, Hens N, Vael C, et al. Differences in gut microbiota composition between obese and lean children: a cross-sectional study. Gut Pathog. 2013;5(1):10. 
51. Harach T, Marungruang N, Duthilleul N, Cheatham V, Mc Coy KD, Frisoni G, et al. Reduction of Abeta amyloid pathology in APPPS1 transgenic mice in the absence of gut microbiota. Sci Rep. 2017;7:41802.

52. Cupidi C, Frangipane F, Gallo M, Clodomiro A, Colao R, Bernardi L, et al. Role of Niemann-Pick Type C Disease Mutations in Dementia. J Alzheimers Dis. 2017;55(3):1249-59.

53. Malnar M, Hecimovic S, Mattsson N, Zetterberg H. Bidirectional links between Alzheimer's disease and Niemann-Pick type C disease. Neurobiol Dis. 2014;72 Pt A:37-47.

54. Kresojevic N, Dobricic V, Svetel M, Kostic V. Mutations in Niemann Pick type C gene are risk factor for Alzheimer's disease. Med Hypotheses. 2014;83(5):559-62.

55. Jeurissen ML, Walenbergh SM, Houben T, Hendrikx T, Li J, Oligschlaeger Y, et al. Myeloid DLL4 Does Not Contribute to the Pathogenesis of Non-Alcoholic Steatohepatitis in Ldlr-/- Mice. PLoS One. 2016;11(11):e0167199.

56. Laukens D, Brinkman BM, Raes J, De Vos M, Vandenabeele P. Heterogeneity of the gut microbiome in mice: guidelines for optimizing experimental design. FEMS Microbiol Rev. 2016;40(1):117-32.

57. Bieghs V, Wouters K, van Gorp PJ, Gijbels MJ, de Winther MP, Binder CJ, et al. Role of scavenger receptor $\mathrm{A}$ and CD36 in diet-induced nonalcoholic steatohepatitis in hyperlipidemic mice. Gastroenterology. 2010;138(7):2477-86, 86 e1-3.

58. Lutjohann D, Stroick M, Bertsch T, Kuhl S, Lindenthal B, Thelen K, et al. High doses of simvastatin, pravastatin, and cholesterol reduce brain cholesterol synthesis in guinea pigs. Steroids. 2004;69(6):431-8.

59. Bonder MJ, Tigchelaar EF, Cai X, Trynka G, Cenit MC, Hrdlickova B, et al. The influence of a short-term gluten-free diet on the human gut microbiome. Genome Med. 2016;8(1):45.

60. Mack I, Cuntz U, Gramer C, Niedermaier S, Pohl C, Schwiertz A, et al. Weight gain in anorexia nervosa does not ameliorate the faecal microbiota, branched chain fatty acid profiles, and gastrointestinal complaints. Sci Rep. 2016;6:26752.

61. Brandt BW, Bonder MJ, Huse SM, Zaura E. TaxMan: a server to trim rRNA reference databases and inspect taxonomic coverage. Nucleic Acids Res. 2012;40(Web Server issue):W82-7.

62. Caporaso JG, Kuczynski J, Stombaugh J, Bittinger K, Bushman FD, Costello EK, et al. QIIME allows analysis of high-throughput community sequencing data. Nat Methods. 2010;7(5):335-6.

63. $\mathrm{R}$ Core Team. R: A language and environment for statistical computing. $\mathrm{R}$ Foundation for Statistical Computing V, Austria. URL: http://www.R-project.org/ (2013).

64. Arumugam M, Raes J, Pelletier E, Le Paslier D, Yamada T, Mende DR, et al. Enterotypes of the human gut microbiome. Nature. 2011;473(7346):174-80.

65. Jari Oksanen FGB, Michael Friendly, Roeland Kindt, Pierre Legendre, Dan McGlinn, Peter R. Minchin, R. B. O'Hara, Gavin L. Simpson, Peter Solymos, M. Henry H. Stevens, Eduard Szoecs, Helene Wagner. 2011. Available from: https://cran.rproject.org/web/packages/vegan/. Accessed on:

66. Maechler M, Rousseeuw, P., Struyf, A., Hubert, M., Hornik, K. Cluster analysis basics and extensions. R package version 2.0.1. 2012.

67. PJ R. Silhouettes: A graphical aid to the interpretation and validation of cluster analysis. Journal of Computational and Applied Mathematics. 1987:53-65. 
Hematopoietic Npc1 mutation shifts gut microbiota composition in Ldlr-/- mice on a high-fat, highcholesterol diet.

68. Clarke KR. Nonparametric Multivariate Analyses of Changes in Community Structure. Australian Journal of Ecology. 1993;18(1):117-43. 


\section{SUPPLEMENTARY MATERIALS}
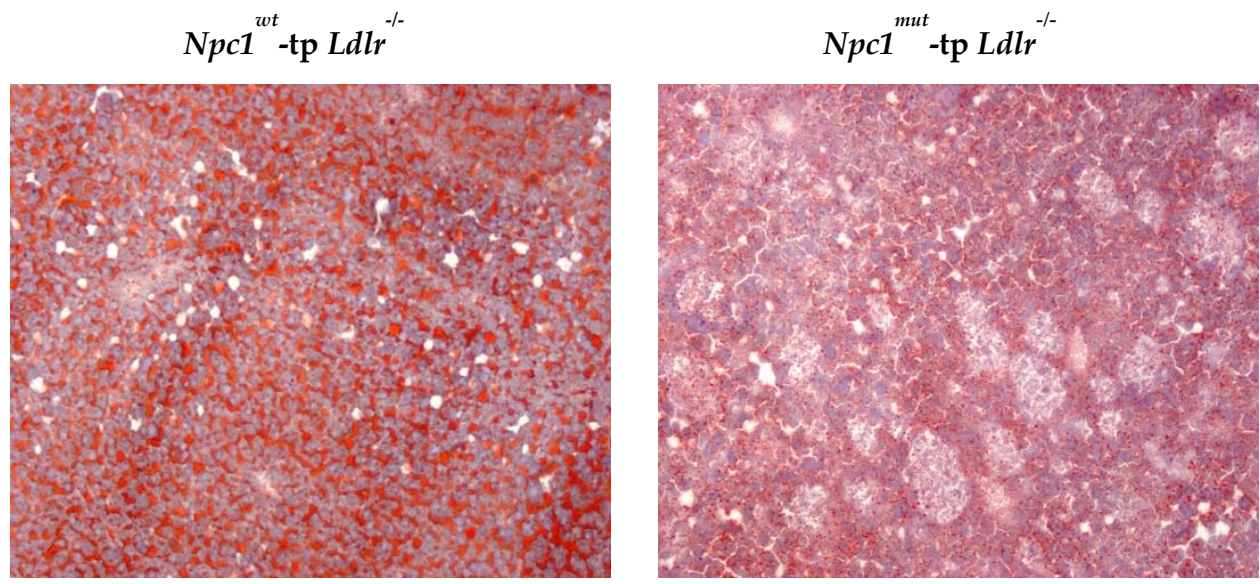

Supplementary Figure S1: Oil Red O staining.

$$
N p c 1^{w t}-\mathrm{tp} L d l r^{-/-}
$$

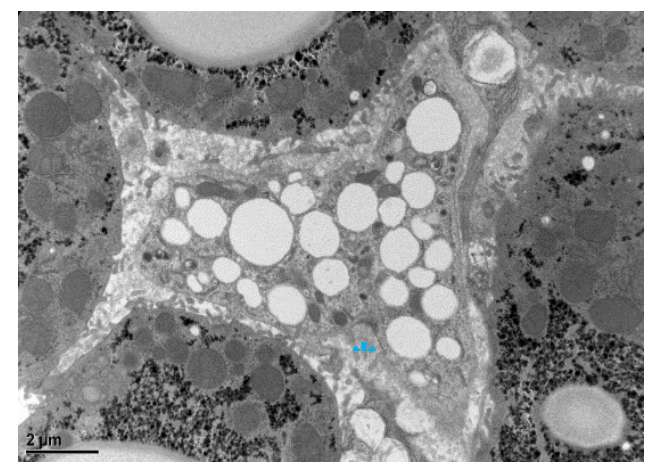

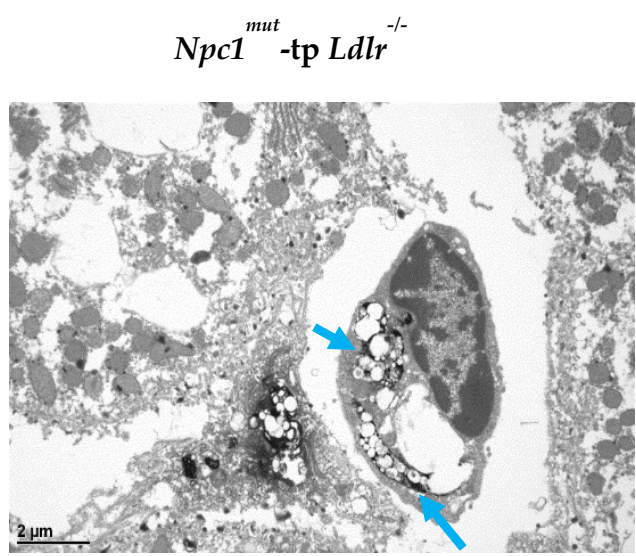

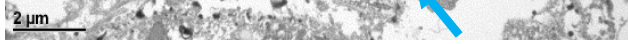

Supplementary Figure S2: Representative images of electron microscope wherein acid phosphatase staining was used for visualisation of lysosomes (blue asterisk and arrows) in hepatic macrophages 
Hematopoietic Npc1 mutation shifts gut microbiota composition in Ldl-/- mice on a high-fat, highcholesterol diet.

A

B
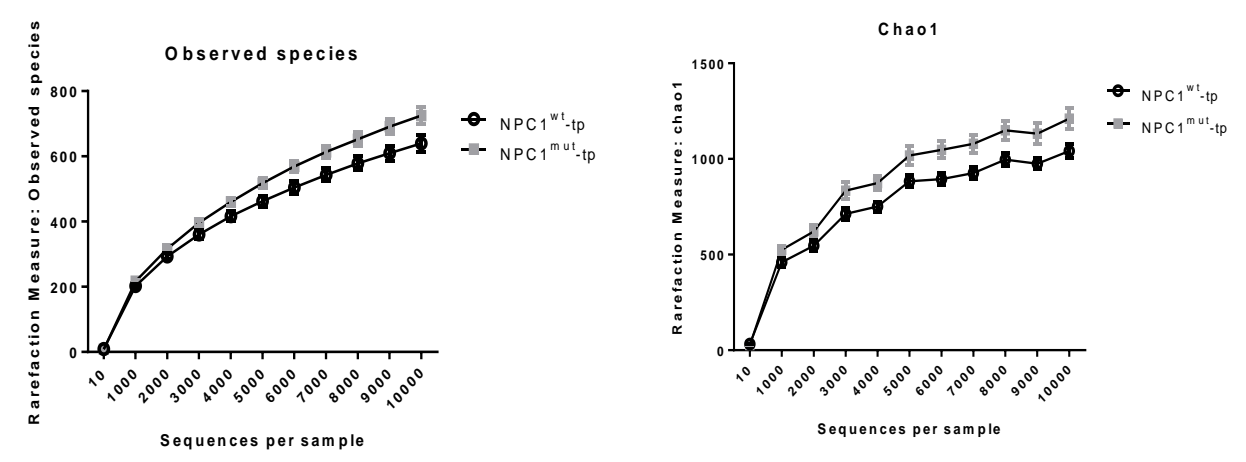

C

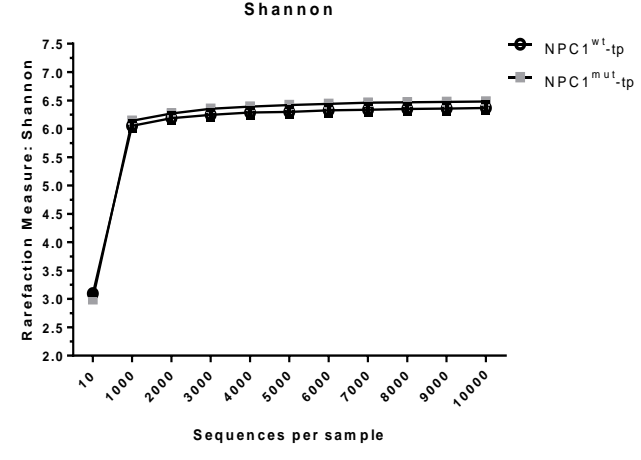

Supplementary Figure S3: Rarefaction plots.

99 



\section{Chapter 5}

\section{Pneumococcal Immunization Reduces Neurological and Hepatic Symptoms in a Mouse Model for Niemann-Pick Type C1 Disease.}

Tom Houben+, Inês Magro dos Reis', Yvonne Oligschlaeger, Hellen Steinbusch, Marion J J Gijbels, Tim Hendrikx, Christoph J Binder, David Cassiman, Marit Westerterp, Jos Prickaerts, Ronit Shiri-Sverdlov

† T.H. and I.M.R. contributed equally to this work.

Front Immunol, 2019; 9:3089 


\begin{abstract}
Niemann-Pick type C1 (NPC1) disease is caused by a deleterious mutation in the Npc1 gene, causing lysosomal accumulation of unesterified cholesterol and sphingolipids. Consequently, NPC1 disease patients suffer from severe neurovisceral symptoms which, in the absence of effective treatments, result in premature death. NPC1 disease patients display increased plasma levels of cholesterol oxidation products such as those enriched in oxidized lowdensity lipoprotein (oxLDL), a pro-inflammatory mediator. While it has been shown that inflammation precedes and exacerbates symptom severity in NPC1 disease, it is unclear whether oxLDL contributes to NPC1 disease progression. In this study, we investigated the effects of increasing anti-oxLDL IgM autoantibodies on systemic and neurological symptoms in an NPC1 disease mouse model. For this purpose, Npc1nih mice were immunized with heat-inactivated S. pneumoniae, an immunogen which elicits an IgM autoantibody-mediated immune response against oxLDL. Npc1nih mice injected with heat-inactivated pneumococci displayed an improved hepatic phenotype, including liver lipid accumulation and inflammation. In addition, regression of motor skills was delayed in immunized Npc1nih. In line with these results, brain analyses showed an improved cerebellar phenotype and neuroinflammation in comparison with control-treated subjects. This study highlights the potential of the pneumococcal immunization as a novel therapeutical approach in NPC1 disease. Future research should investigate whether implementation of this therapy can improve life span and quality of life of NPC1 disease patients.
\end{abstract}




\section{INTRODUCTION}

Niemann-Pick type C1 (NPC1) disease is a rare, fatal disorder caused by mutations in the Npc1 gene which result in the accumulation of unesterified cholesterol and glycosphingolipids in late endosomes/early lysosomes (1-3). The earliest clinical findings of NPC1 disease disease are mostly related to systemic problems in the liver (neonatal jaundice, liver failure (4-6)) and the spleen (splenomegaly and severe abdominal pain resulting in splenectomies (7) and increased sensitivity to spontaneous infections (8)). While, in most patients, the systemic symptoms resolve over time, some develop severe systemic problems resulting in premature death (4). Besides the systemic manifestation of the disease, NPC1 disease is also characterized by severe disturbances in the central nervous system leading to the progressive impairment of motor and cognitive function (2). In spite of significant advances in the development of therapeutic interventions for NPC1 disease $(9,10)$, there is currently no curative treatment available.

While lysosomal lipid accumulation is at the root of NPC1 disease, secondary pathological mechanisms such as oxidative stress (11), apoptosis (12) and inflammation $(13,14)$ have been shown to accompany and even contribute to NPC1 disease progression. Similarly, atherosclerosis and non-alcoholic steatohepatitis (NASH) also feature the aforementioned disease mechanisms $(15,16)$ and lysosomal lipid accumulation in macrophages (17). These observations suggest a mechanistic link between the pathological mechanisms of NPC1 disease, atherosclerosis and NASH. Of note, increasing anti-oxLDL IgM autoantibody levels in the latter metabolic diseases ameliorates the macrophage-mediated inflammatory reaction (18-20). Relevantly, multiple research papers have described the cholesterol oxidation products 7 -ketocholesterol and cholestane-3 $\beta, 5 \alpha, 6 \beta$-triol, two products abundantly present in oxLDL $(21,22)$, as sensitive and specific blood-based biomarkers for diagnosing NPC1 disease $(6,23,24)$. However, whether these cholesterol oxidation products contribute to NPC1 disease progression has to our knowledge never been investigated.

Given the mechanistic overlap between the pathologies of NPC1 disease and of the metabolic diseases atherosclerosis and NASH, the aim of the current study was to determine whether increasing anti-oxLDL IgM autoantibodies reduces NPC1 disease symptoms. For this purpose, $N p c 1^{\text {ih }}$ mice were immunized with heat-inactivated Streptococcus pneumoniae. $N p c 1^{\text {nih }}$ mice are a well-established NPC1 disease model in which a mutation in Npc1 leads to NPC1 truncation and loss of function (25). This mouse model recapitulates some of NPC1 disease most prominent features, including severe lysosomal cholesterol accumulation in most organs and motor function deficits (26-30). Immunization of mice using inactivated $S$. pneumoniae has been established as an effective approach to increase serum titers of anti- 
oxLDL IgM autoantibodies by means of molecular mimicry $(18,31)$. In line with our hypothesis, immunized $N p c 1^{\text {nih }}$ mice showed reduced motor function decay over time, decreased neuro- inflammation and degeneration, reduced inflammation in liver and spleen and an improvement in liver lipid metabolism. These findings provide evidence for the involvement of anti-oxLDL IgM autoantibodies in NPC1 disease progression and highlight vaccination strategies leading to the elevation of anti-oxLDL IgM titers as a promising therapeutic approach to reduce NPC1 disease symptoms. 


\section{MATERIALS AND METHODS}

\section{Preparation of immunogen}

For immunization, the heat-inactivated R36A strain of S. pneumoniae (Birmingham, AL) was used, still bearing the PC headgroup epitope similar to oxLDL. Colonies of the R36A strain were harvested at mid-log phase after incubation at $37^{\circ} \mathrm{C}$ on blood agar plates and transferred to Todd-Hewitt plus $0.5 \%$ yeast broth. The mid-log phase is characterized by an optical density (OD) value of 0.425 to 0.45 at $600 \mathrm{~nm}$. S. pneumoniae were heat-inactivated at $60^{\circ} \mathrm{C}$ for 30 minutes; afterwards, no colonies of this suspension were detected on blood agar plates, thus confirming their inactivation. For freezer stocks of strain R36A, small aliquots of $S$. pneumoniae at mid-log density were harvested and suspended in Todd-Hewitt plus $80 \%$ sterile glycerol and stored at $-80^{\circ} \mathrm{C}(32)$.

\section{Mice and immunization}

$N p c 1^{\text {nih }}$ mice were housed under standard conditions and were given free access to food and water. A complementary experiment with age-matched wildtype mice was performed to confirm the phenotypical effect of the Npc1 mutation in $N p c 1^{\text {nih }}$ mice. Experiments were performed according to Dutch laws and were approved by the Animal Experiment Committee of Maastricht University. $N p c 1^{\text {nih }}$ mice were derived from heterozygous founders (C57BL/6 / Npc1 ${ }^{\text {nih }}$ ).

The immunization protocol started in 2-week old $N p c 1^{\text {nih }}$ mice (male or female) fed a normal chow diet. Mice were divided into 2 groups ( $\mathrm{n}=12$ for both groups). One of the groups received the equivalent of $10^{8}$ colony-forming units of heat-inactivated pneumococcal immunogen emulsified in $200 \mu \mathrm{l}$ of sterile $0.9 \% \mathrm{NaCl}$ for the primary subcutaneous immunization; subsequently, two intraperitoneal booster immunizations were administered every 2 weeks (31). The control group received $\mathrm{NaCl}$ injections. During the experiment, which lasted 5 weeks in total, mice were given a normal chow diet. Blood from the tail vein was collected at week 3 and 5. An overview of the experimental set-up is given in Fig. S1. During the course of the study, one control-treated $N p c 1^{\text {nih }}$ mouse died, and was therefore excluded from the experimental analyses. All tissues were isolated and snap-frozen in liquid nitrogen and stored at $-80^{\circ} \mathrm{C}$ or fixed in $4 \%$ formaldehyde/PBS. The collection of blood and tissue specimens, RNA isolation, cDNA synthesis and qPCR were determined as described previously $(18,33,34)$. Also the measurements of the autoantibody titers against IgG and IgM antibodies to $\mathrm{CuOx}-\mathrm{LDL}$ and PC-BSA have been described extensively (18).

\section{Lipid measurements}

Total plasma cholesterol and triglyceride levels were measured (1489232, Chol CHOD-PAP, Roche, Almere, the Netherlands; 337-B, TG GPO-trinder, Sigma Aldrich, Zwijndrecht, the 
Netherlands). Measurements were done according to manufacturer's protocols on a Benchmark 550 Micro-plate Reader (170-6750XTU, Bio-Rad, Veenendaal, the Netherlands). For organ lipid analyses, approximately $50 \mathrm{mg}$ of frozen liver was homogenized for 30s at 5000rpm in a closed tube with 5.0mm glass beads and $1.0 \mathrm{ml}$ SET buffer (Sucrose $250 \mathrm{mM}$, EDTA $2 \mathrm{mM}$ and Tris $10 \mathrm{mM}$ ) (35). Complete cell destruction was done by two freeze-thaw cycles and 3 times passing through a 27- gauge syringe needle and a final freeze-thaw cycle. Protein content was measured with the BCA method (23225, Pierce, Rockford, IL, USA). TG were measured as described above. All analyses were performed according to manufacturers' instructions.

\section{Immunohistochemistry}

Frozen liver sections $(7 \mu \mathrm{m})$ were fixed in acetone and blocked for endogenous peroxidase by incubation with $0.25 \%$ of $0.03 \% \mathrm{H}_{2} \mathrm{O}_{2}$ for 5 minutes. Primary antibodies used were against neutrophils (rat anti-mouse Ly6C, clone NIMP-R14), hepatic macrophages (rat anti-mouse CD68, clone FA11) and infiltrated macrophages and neutrophils (rat anti-mouse Mac-1 [M1/70]). 3-Amino-9-ethylcarbazole (AEC) was applied as color substrate and hematoxylin for nuclear counterstain. Sections were enclosed with Faramount aqueous mounting medium.

Paraffin-embedded cortical and cerebellar sections ( $4 \mu \mathrm{m}$; sagitally) were deparaffinized and stained with hematoxylin-eosin (HE) and primary antibodies against Iba-1 and calbindin. Sections were subjected to heat-mediated antigen retrieval and blocking with $4 \%$ goat serum/PBS, after which they were incubated with rabbit anti-Iba1 overnight. The following day, sections were incubated with swine anti-rabbit, whose signal was amplified with the VECTASTAIN® Elite ${ }^{\circledR}$ ABC system and counterstained with a DAB solution. For calbindin immunostaining, sections' endogenous peroxidase activity was blocked by incubating in $0.5 \%$ $\mathrm{H}_{2} \mathrm{O}_{2} /$ methanol for 10 minutes. After performing heat-mediated antigen retrieval, sections were incubated with rabbit anti-calbindin overnight. The following day, sections were incubated with biotinylated goat anti-rabbit antibody, whose signal was amplified by using the VECTASTAIN® Elite® ABC system.

Pictures were taken with a Nikon digital camera DMX1200 and ACT-1 v2.63 software (Nikon Instruments Europe, Amstelveen, The Netherlands).

Cerebellar Purkinje cells (calbindin) were counted and scored in ten to twenty microscopical views (original magnification, 200x). Number of Purkinje cells was indicated as number of cells per pixel (Adobe Photoshop CS2 v.9.0.). Scoring was performed according to presence of Purkinje cells' dendritic structure (1, defined arborization structure; 2, intermediary; 3, lack 
of arborization structure). Purkinje cells' number and scoring are derived from the average of independent observations from two blinded researchers.

Cerebellar Purkinje cells' HE histological staining was assessed in 10 microscopical views (original magnification, 100x) by quantifying HE staining using Fiji plugin of ImageJ software (v. 1.52h).

Cortical microglia (Iba-1) were counted and scored in twelve microscopical views (original magnification, 400x) and indicated as number of cells per field. Microglia were scored according to their phenotype as follows: type 1 (characterized by small cell bodies and long, thin fillaments); type 2 (thicker and less numerous fillaments); type 3 (large cell body, no fillaments) (Fig. S2) (36). Microglia numbers and scoring are given as the average of independent analyses done by two blinded researchers.

Hepatic foamy cells (HE) were assessed in five microscopical views (original magnification, 200x) by an experienced pathologist and given a score in arbitrary units (A.U). Splenic fat accumulation (HE) was evaluated in three microscopical views (original magnification, 200x) by quantifying the percentage of positive area using Adobe Photoshop CS2 v.9.0.

Hepatic neutrophils (NIMP) and infiltrated macrophages and neutrophil cells (Mac-1) were counted in six microscopical views (original magnification, 200x) and were indicated as number of cells per square millimeter (cells $/ \mathrm{mm}^{2}$ ). Immunostainings for hepatic macrophages (CD68) were evaluated by an experienced pathologist and given a score in arbitrary units (A.U.).

\section{Genotyping}

Genotypes of animals were determined by PCR analysis of tail DNA. Tails were clipped at postnatal day 6 and homogenized in DirectPCR-Tail (Peqlab, Erlangen, Germany) supplemented with a tenth part Proteinase K (Qiagen, Hilden, Germany). Three hours of incubation at $56^{\circ} \mathrm{C}$ and agitation at 1000 rounds per minute on a Thermo Mixer were followed by $45 \mathrm{~min}$ of heating at

$85^{\circ} \mathrm{C}$ to inactivate the proteinase. Samples were then spun at full speed in a benchtop centrifuge for $1 \mathrm{~min}$. The PCR reactions were performed with $0.5 \mathrm{ml}$ of the obtained extracts. Each lysate underwent two PCRs; Primers gccaagtaggcgacgact and catctactgggtctccatatgtat identified the wild-type allele and primers gccaagtaggcgacgact and ttccaattgtgatctttccaa identified the mutant allele. Both PCRs were carried out under similar cycling conditions. 


\section{Pole test}

The pole test was performed as previously described with minor modifications $(37,38)$. The mouse was placed head-upward on a small platform on top of a vertical rough-surfaced pole (diameter $10 \mathrm{~mm}$; height $50 \mathrm{~cm}$ ) and the time until the mouse descended to the floor (locomotor activity time: TLA) was recorded with a maximum duration of $120 \mathrm{~s}$. Even if the mouse descended part of the way and fell the rest of the way, the behavior was scored until it reached to the floor. When the mouse did not turn downward and instead dropped from the pole, TLA was considered as $120 \mathrm{~s}$ because of maximal severity. The pole test was performed on 3 different days (day 18, 25 and 32), with 5 consecutive trials per mouse.

\section{Accelerod (accelerated rotarod) test}

Balance and motor coordination of mice was evaluated using accelerod performance on day 21 and day 33 of the experiment. The accelerod system for mice was used under standard room conditions. The apparatus consisted of a base platform and a rotating rod with a grooved surface. Before accelerod testing, mice were trained at 2 separate days (day 17 and 19 of the experiment). On both training days, mice underwent 3 separate training moments (separated 2 hours from eachother) in which they endured 3 trials with a constant speed of 18 revolutions per minute for $120 \mathrm{~s}$. When operated in the acceleration modus, the rotation increased from 4 to 40 revolutions per minute in $30 \mathrm{~s}$ steps within $5 \mathrm{~min}$. The performance was measured as the latency to fall (s).

\section{Statistical analysis}

Data were statistically analysed by performing two-tailed nonpaired $t$ test, two-way ANOVA followed by Tukey's post-hoc correction and repeated measures two-way ANOVA followed by Tukey's post-hoc correction using GraphPad Prism version 6 for Windows. Data were expressed as the mean and standard error of the mean. Data were considered significantly different compared to control-treated $N p c 1^{\text {nih }}$ mice, except for the analyses of rotarod and pole test results, in which comparisons were made between different time points among the same study groups. $\left({ }^{*} p \leq 0.05 ;{ }^{* *} p<0.01 ;{ }^{* * *} p<0.001 ;{ }^{* * * *} p<0,0001\right)$. 


\section{RESULTS}

$N p c 1^{\text {nih }}$ mice recapitulate NPC1 disease features and display elevated anti-oxLDL IgM autoantibody titers after immunization with heat-inactivated pneumococci

To assess the effects of the Npc1 nith mutation, body, spleen and liver weight were compared between control-treated $N p c 1^{\text {nih }}$ mice and age-matched wildtype mice. Whereas total spleen weight was reduced in $N p c 1^{\text {inh }}$ mice compared to wildtype mice, total liver weight was increased in Npc1 ${ }^{\text {nih }}$ mice (Table S1). As expected, body weight of $N p c 1^{\text {nih }}$ mice was reduced in comparison with age-matched wildtype mice (Table S1), reflecting overall NPC1 disease burden. To determine whether anti-oxLDL IgM autoantibodies have a protective effect in NPC1, two week-old Npc1 ${ }^{\text {nih }}$ mice were immunized with heat-inactivated pneumococci, known to induce high anti-oxLDL IgM titers dominated by T15-idiotypic $\operatorname{IgM}(18,31)$. While total body weight (Fig. S3A) and total liver weight did not differ (Fig. S3B), total spleen weight was decreased in immunized $N p c 1^{\text {nih }}$ mice compared to control-treated counterparts (Fig. $\mathrm{S} 3 \mathrm{C})$. As expected, immunization resulted in a strong increase in plasma anti-oxLDL antibody levels in $N p c 1^{\text {nih }}$ mice (Fig. 1A, B). Only weak IgG responses were observed, which is in line with previous reports showing pneumococcal immunizations triggering an IgM-dominated thymus-independent type-2 response highly specific for phosphorylcholine (PC) (Fig. 1C, D).

A

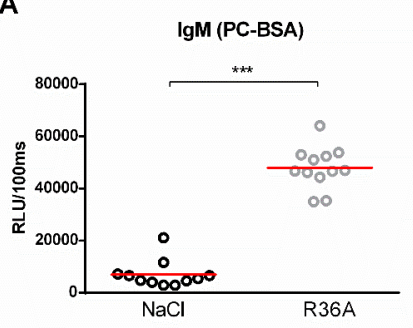

C

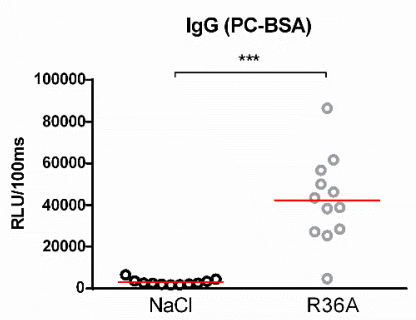

B

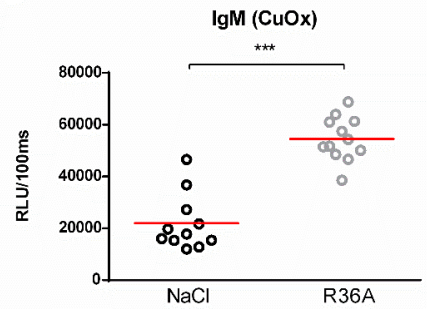

D

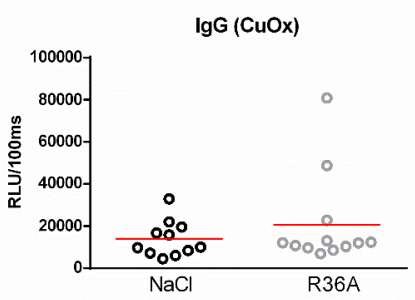

Figure 1: IgM autoantibodies in Npc1 $1^{\text {ih }}$ mice receiving control or heat-inactivated pneumococci injections. (A-D) IgM and IgG antibodies against oxLDL (CuOx and PC-BSA) were measured in the plasma of control $(\mathrm{n}=11)$ and pneumococci-immunized $(\mathrm{n}=12) N p c 1^{\text {nih }}$ mice. Data are expressed as relative light units RLU/100 ms and were triplicate determinations. ${ }^{* * *}$ Indicates $\mathrm{p}<0.001$ compared to $\mathrm{NaCl}$-treated $N p c 1^{\text {nih }}$ mice by use of two-tailed unpaired $\mathrm{t}$ test. $\mathrm{n}=$ 11-12 mice per group. All error bars are SEM. 
Delayed motor skill degeneration and improved neuroinflammation and cerebral morphology in immunized $N p c 1^{\text {nih }}$ mice

Motor skill degeneration is a common neurological feature of NPC1, due to the progressive loss of Purkinje cells. To determine the effects of the pneumococcal immunization on motor function of $N p c 1^{\text {ih }}$ mice, pole and rotarod tests were performed throughout the study period. As expected, $N p c 1^{\text {nih }}$ mice performance on both tests was consistently worse than their wildtype counterparts (Table S1), confirming NPC1 disease burden on motor function. By the end of the study, control-treated $N p c 1^{\text {nih }}$ mice took significantly longer time to descend the pole (Fig. 2A), whereas locomotor activity time (TLA) of immunized $N p c 1^{\text {in }}$ mice remained stable over the entire experiment. Additionally, the motor function scores obtained for the pole test strongly correlate with the levels of plasma anti-oxLDL IgM autoantibodies, suggesting a link between the amount of circulating anti-oxLDL IgM autoantibodies and overall motor performance (Fig. S4). While the performance of both control-treated and immunized $N p c 1^{\text {nih }}$ mice on the rotarod test worsened significantly over time (Fig. 2B), the observed performance decay was more prominent in the control-treated $N p c 1^{\text {nih }}$ mice. These results indicate that motor function deterioration over time was decreased in immunized $N p c 1^{\text {nih }}$ mice.

Next, we investigated whether pneumococcal immunization affects Purkinje cells in Npc1 ${ }^{\text {nih }}$ mice. In line with motor skill function results, the integrity of cerebella of control-treated $N p c 1^{n \text { ih }}$ was severely compromised, whereas immunized $N p c 1^{\text {nih }}$ mice retained more of their cerebellar structure (Fig. 2C-D; Fig. S5A). Furthermore, Purkinje cells of untreated Npc1 ${ }^{\text {nih }}$ mice displayed increased hematoxylin and eosin (HE) staining intensity, suggesting higher activation levels due to damage (Fig. 2E, upper panel; Fig. 2F; Fig. S5B). Overall, these results indicate that pneumococcal immunization delays cerebellar neurodegeneration in $N p c 1^{\text {nih }}$ mice. In addition to severe cerebellar neurodegeneration, cortical immune cells were observed in control-treated $N p c 1^{\text {nih }}$ mice, in contrast with immunized $N p c 1^{\text {iih }}$ mice, suggesting higher levels of neuroinflammation in the control-treated group (Fig. 2E, lower panel; Fig. S5B).

We further analysed the effects of pneumococcal immunization on neuroinflammation by examining microglial cells using immunohistochemical staining for Iba-1 (Fig. 2G). While no differences were observed in total number of microglia (Fig. $2 \mathrm{H}$ ), microglia of immunized $N p c 1^{\text {nih }}$ mice exhibited larger cell bodies and shorter, less ramified processes, suggesting a change in microglia phenotype following pneumococcal immunization (Fig. 2I).

Finally, we analysed cerebral and cerebellar gene expression analysis of the inflammatory markers C-X-C motif chemokine 10 (Cxcl10), chemokine (C-C motif) ligand 3 ( $\mathrm{Ccl} 3$ ) and 
cluster of differentiation 68 (Cd68). Immunization of $N p c 1^{\text {nih }}$ mice reduced expression of all inflammatory markers in the cerebrum, with the exception of $\mathrm{Ccl3}$, in which a trend suggesting decreased expression was observed (Fig. 2J). In addition, while expression of Cxcl10 and Cd68 in the cerebellum tended to be decreased, expression of $\mathrm{Ccl} 3$ was reduced in the cerebellum of immunized Npc1 $1^{\text {nih }}$ mice (Fig. 2K). Furthermore, gene expression of cathepsin D (Ctsd), a marker previously linked to NPC1 severity in mice $(39,40)$, and Npc2, which is known to bind to cholesterol in the lysosome, were reduced in the cerebrum of $\mathrm{Npc} 1^{\text {nih }}$ mice that received the immunization, indicating an improved neurological phenotype after immunization. Notably, cerebral and cerebellar expression of several of these markers correlated with the plasma anti-oxLDL IgM autoantibody levels (Fig. S4), underlining the link between the neurological phenotype and anti-oxLDL IgM autoantibody levels. Altogether, these results indicate beneficial effects of immunization with heat-inactivated pneumococci on the neurological phenotype of $N p c 1^{\text {nih }}$ mice.

\section{Decreased hepatic and splenic lipid levels in immunized $N p c 1^{\text {nih }}$ mice}

To determine whether immunizing $N p c 1^{\text {nih }}$ mice with heat-inactivated pneumococci influences hepatic lipid levels, biochemical assessment of hepatic cholesterol and triglycerides was performed. $\mathrm{Npc}^{\text {nih }}$ mice showed an increase in plasma and liver cholesterol levels compared to age matched wildtype mice (Table S1). Seven week-old Npc1 ${ }^{\text {ih }}$ mice that received the immunization demonstrated reduced levels of hepatic total cholesterol (Fig. 3A) and triglycerides (Fig. 3B) compared to control-treated $N p c 1^{\text {ih }}$ mice, though the latter only showed a trend. In line, HE-staining of the liver confirmed the decrease of lipid levels in hepatocytes and the reduced foamy appearance of hepatic macrophages in immunized $\mathrm{Npc} 1^{\text {ih }}$ mice (Fig. 3C-D). Relevantly, hepatic gene expression levels of acetyl-CoA acetyltransferase 2 (Acat2), the enzyme responsible for cholesterol esterification, were elevated in immunized $N p c 1^{\text {nih }}$ mice, suggesting increased cholesterol esterification upon immunization (Fig. 3E). In contrast, no differences were observed in plasma lipid levels between control-treated and immunized $N p c 1^{\text {nih }}$ mice (Fig. 3F-G). To obtain a more detailed view on hepatic lipid distribution, electron microscopy analysis was performed (Fig. $3 \mathrm{H})$. Though both control and immunized $\mathrm{N} p c 1^{\text {nih }}$ mice showed the NPC1 characteristics of hepatic lipid inclusions (blue circles) and vacuoles with electron-dense material (asterisk), immunized $N p c 1^{\text {nih }}$ mice demonstrated a less foamy appearance of hepatic macrophages (arrow). In contrast with these observations, no differences were observed in lipid accumulation in the spleen of immunized $N p c 1^{\text {nih }}$ mice and control-treated Npc1 ${ }^{\text {nih }}$ mice (Fig. 3I-J). Overall, these results suggest improved hepatic lipid metabolism after increasing anti-oxLDL IgM autoantibody levels in $\mathrm{Npc}^{\text {nih }}$ mice. 
A

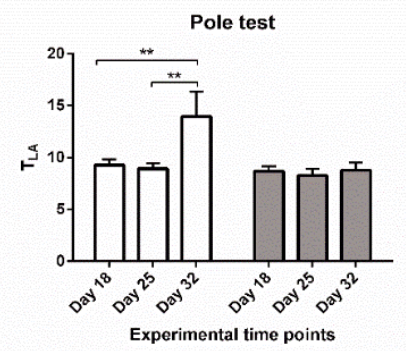

C

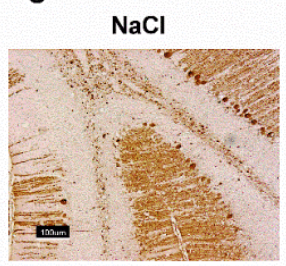

D

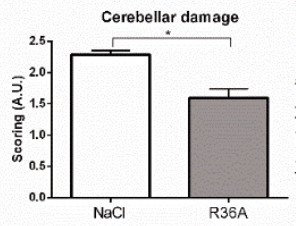

G
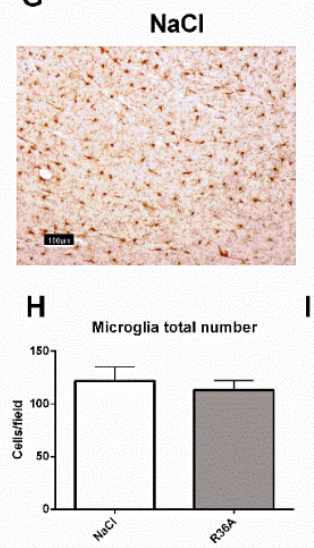

F
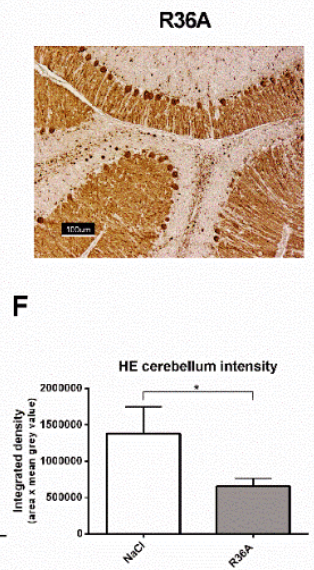

R36A

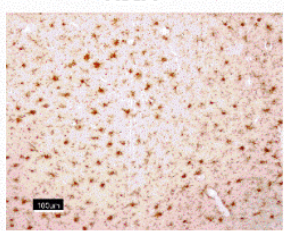

I Microglia phenotype scoring

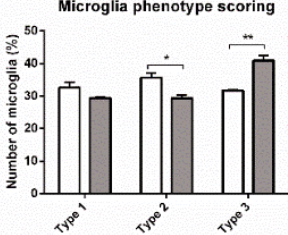

B

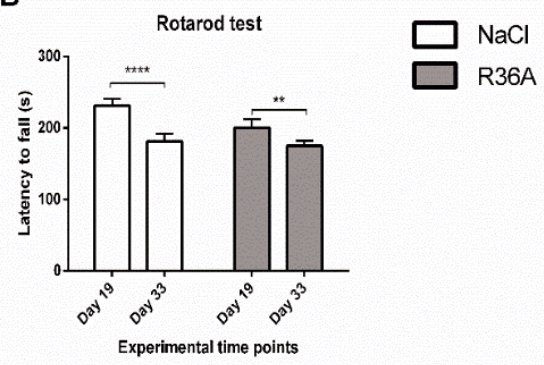

E
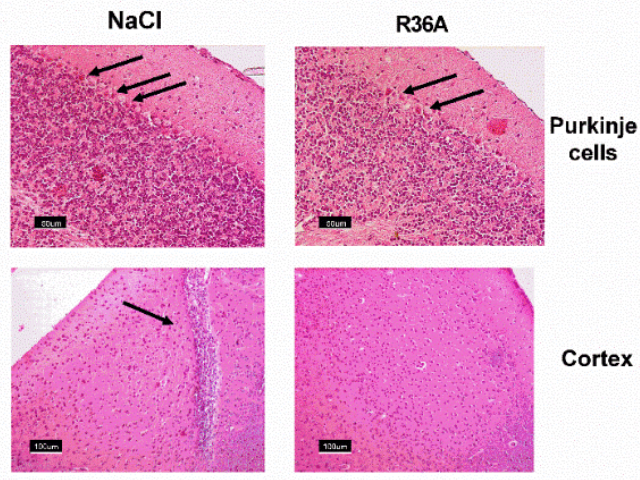

Cortex

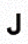

Cerebrum

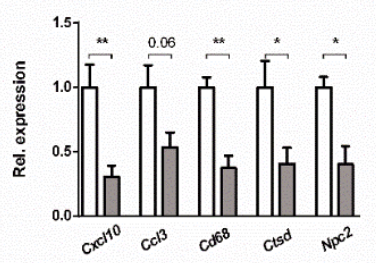

K

Cerebellum

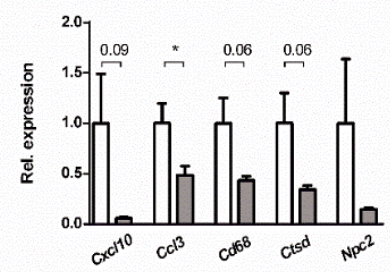

Figure 2: Neurological parameters. (A) Locomotor activity time (TLA) of control and immunized Npc1 ${ }^{\text {nih }}$ mice as measured by the pole test at day 18, 25 and 32 of the experiment. (B) Rotarod performance of control and immunized $N p c 1^{\text {nih }}$ mice at day 19 and 33 of the experiment. (C, D) Representative pictures and scoring of calbindin staining of Purkinje cells in cerebellum. (E) General histology of the brain by HE staining. Arrows indicate Purkinje cells (top panels) and immune cells (bottom left panel). (F) Quantification of Purkinje HE staining density. (G-I) Representative pictures, quantification and scoring of Iba-1 staining of cortical microglia (type 1, type 2 and type 3 ). (J-K) Gene expression analysis of $\mathrm{Cxcl10}, \mathrm{Ccl} 3, \mathrm{Cd} 68, \mathrm{Npc2}$ and $\mathrm{Ctsd}$ in cerebrum and cerebellum. Data are shown relative to NaCl-treated $N p c 1^{\text {nih }}$ mice 
for all analyses except for pole test and rotarod data, where comparisons were made between different time points within the same experimental group. ${ }^{*}$ Indicates $p<0.05,{ }^{* *} p<0.01$ and ${ }^{* * *} p<0.001$ by use of two-tailed unpaired $t$ test or repeated measures two-way ANOVA with Tukey's post-hoc correction (in pole test and rotarod data analysis only). $\mathrm{n}=11$ 12 mice per group for pole and rotarod tests and $n=5$ mice per group for gene expression data. All error bars are SEM.

\section{Improved hepatosplenic phenotype in immunized $N p c 1^{\text {nih }}$ mice}

To determine whether immunization of $N p c 1^{\text {nih }}$ mice affects hepatic inflammation, hepatic cryosections were stained for the inflammatory cell markers NIMP (neutrophils), CD68 (resident macrophages) and Mac-1 (infiltrated macrophages and neutrophils). The number of infiltrated neutrophils and macrophages in control-treated $N p c 1^{\text {nih }}$ mice was increased compared to wildtype mice, confirming increased hepatic inflammation in $N p c 1^{\text {nih }}$ mice (Table S1). Following pneumococcal immunization, the number of neutrophils and resident macrophages were reduced $N p c 1^{\text {nih }}$ mice (Fig. $4 \mathrm{~A}-\mathrm{B}$ and D). Although the number of infiltrated neutrophils and macrophages (Fig. 4C) and HE-scoring of hepatic inflammation (Fig. 3C and Fig. S6) did not reach statistical significance between study groups, a trend towards reduction of these parameters following immunization was observed. To confirm these histological findings, hepatic gene expression analysis was performed on the inflammatory markers tumor necrosis factor alpha ( $\operatorname{Tnf} \alpha)$, chemokine (C-C motif) ligand 2 (Ccl2), macrophage inflammatory protein 2 (Mip2). Tnfo and Ccl2 expression levels were significantly reduced in immunized $N p c 1^{\text {nih }}$ mice compared to non-immunized $N p c 1^{\text {iih }}$ mice (Fig. 4E). The expression of Mip2 and Ctsd, a marker for disease severity, showed no difference between control-treated and immunized $N p c 1^{\text {iih }}$ mice, although a trend suggesting reduced expression following immunization was observed (Fig. 4E). As increased hepatic apoptosis is linked to inflammation and damage of the liver (41), hepatic apoptosis was also assessed. The amount of apoptotic cells, as quantified by HE-staining, was reduced upon immunization (Fig. 4F), indicating an improvement of the NPC1 disease phenotype in the liver after pneumococcal immunization. Additionally, similar results were observed in the spleen, showing reduced expression of inflammatory markers, although in the case of $\operatorname{Tnf} \alpha$, only a trend was observed (Fig. 5A). Finally, hepatosplenic expression of most of these markers correlated with the plasma anti-oxLDL IgM autoantibody levels (Fig. S4), underlining the link between the systemic improvements and the anti-oxLDL IgM autoantibody levels. Altogether, these findings indicate an improved hepatosplenic phenotype in $N p c 1^{\text {nilh }}$ mice that received the heat-inactivated pneumococci immunization, supporting its potential as treatment for NPC1 disease. 
A

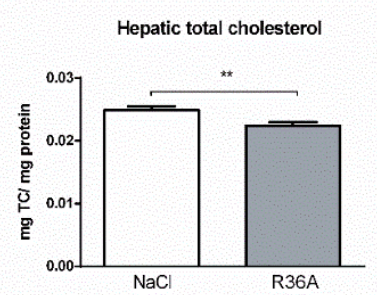

D

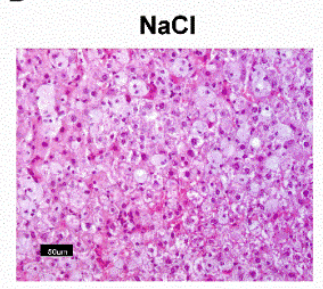

F

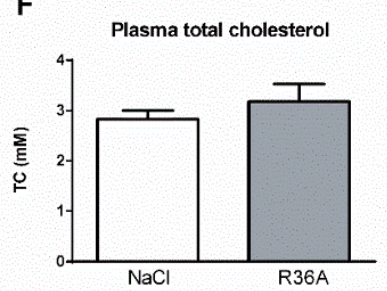

H

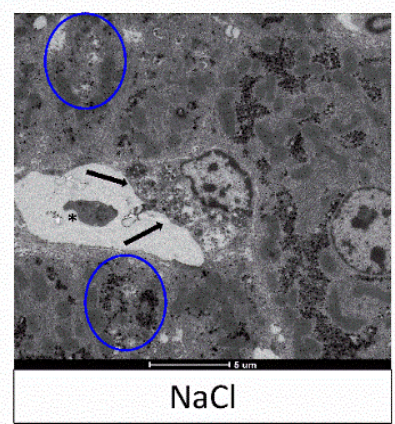

B

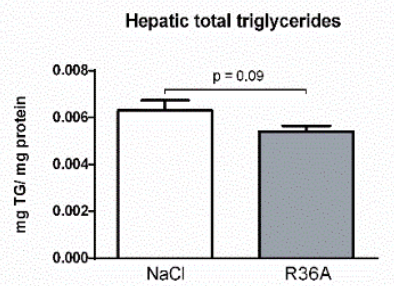

C

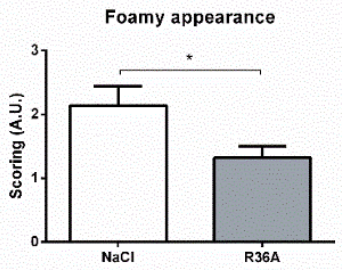

E

R36A

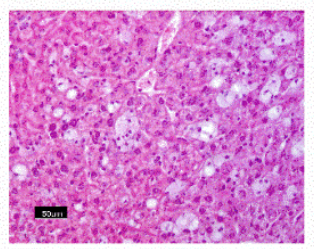

G
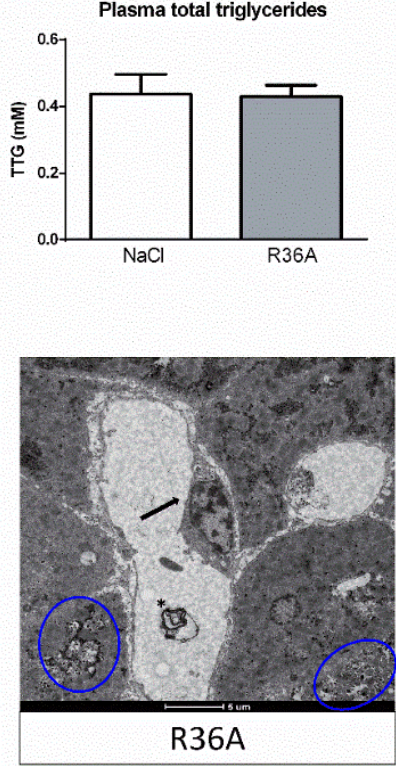

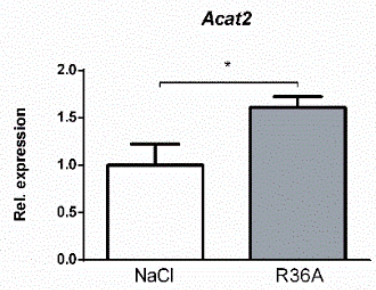

J Splenic fat accumulation

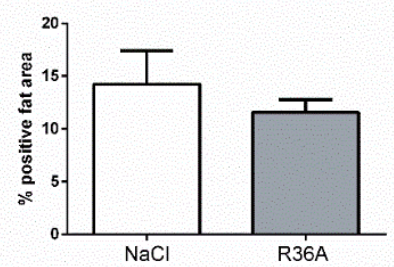

I

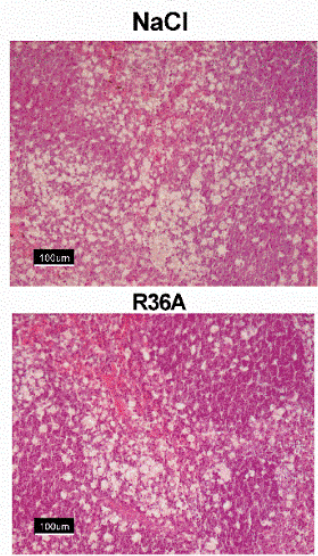

Figure 3: Parameters of lipid metabolism. (A, B) Hepatic total cholesterol and total triglyceride levels of control and immunized $N p c 1^{\text {nih }}$ mice ( $\mathrm{n}=11$ in both experimental groups). (C, D) Scoring and representative pictures of hepatic HEstaining (200x magnification). (E) Hepatic gene expression analysis of Acat2. Data is shown relative to NaCl-treated $N p c 1^{\text {nih }}$ mice. $(F, G)$ Plasma total cholesterol and total triglyceride levels of seven week-old control and immunized Npc1 nih mice. $(\mathrm{H})$ Electron microscopy of livers of control and immunized $N p c 1^{\text {nih }}$ mice. Arrows indicate a hepatic macrophage; blue circles indicate lipid inclusions and asterisks indicate vacuoles with electron-dense material. (I, J) Representative 
pictures and quantification of HE staining of the spleen (100x magnification). ${ }^{*}$ Indicates $p<0.05$ and ${ }^{* *} p<0.01$ compared to $\mathrm{NaCl}$-treated $\mathrm{Npc1} 1^{\text {ih }}$ mice by use of two-tailed unpaired $t$ test. $\mathrm{n}=11-12$ mice per group. All error bars are SEM.

A

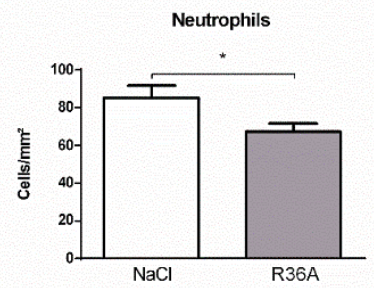

B

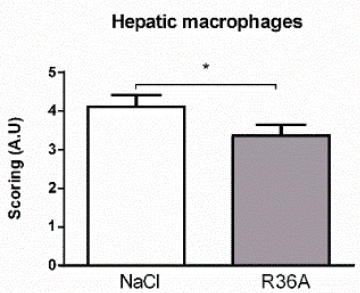

C Infiltrated macrophages and neutrophils

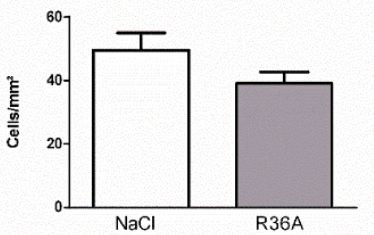

D

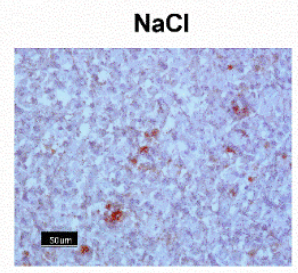

R36A

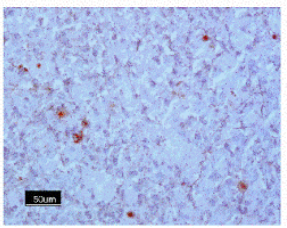

E
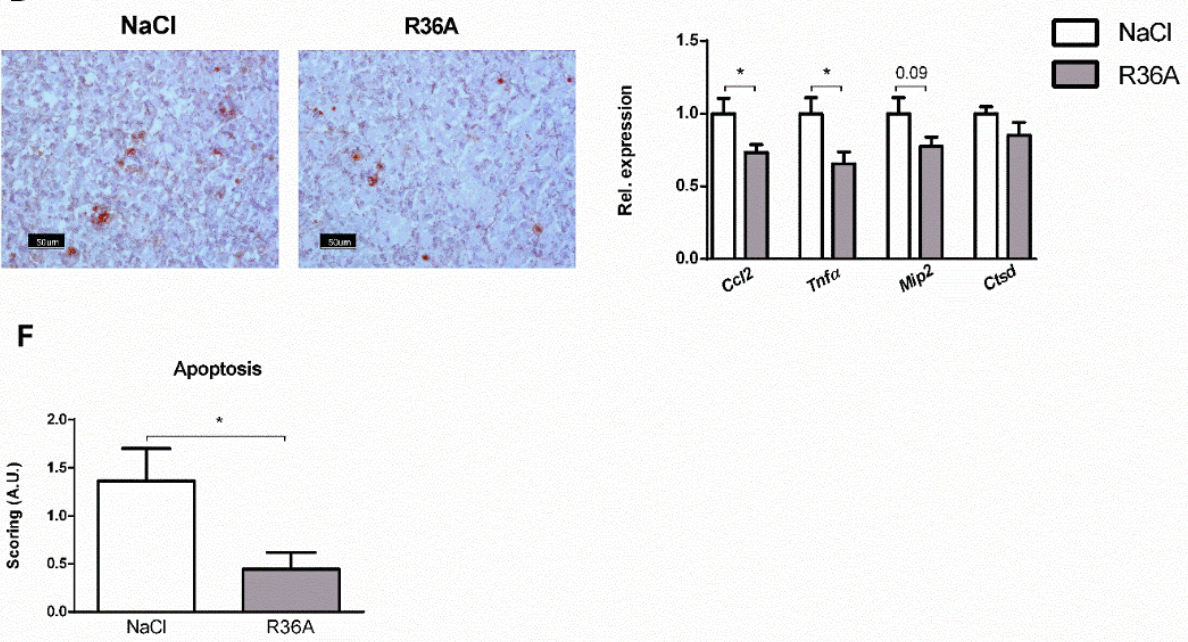

Figure 4: Hepatic parameters. (A-D) Liver sections were stained for neutrophils (NIMP), hepatic macrophages (CD68) and infiltrated macrophages and neutrophils (Mac-1). NIMP and Mac-1 positive cells were counted, whereas the CD68 stainings were scored. Panel D shows representative images of the NIMP staining. (E) Hepatic gene expression analysis of inflammatory markers Ccl2, Tnfo and Mip2. Data are shown relative to NaCl-treated $N p c 1^{\text {nih }}$ mice. (F) Quantification of hepatic apoptosis, assessed by scoring of HE-staining of the liver. ${ }^{*}$ Indicates $p<0.05$ compared to NaCl-treated $N p c 1^{\text {nih }}$ mice by use of two-tailed unpaired $t$ test. n=11-12 mice per group. All error bars are SEM.

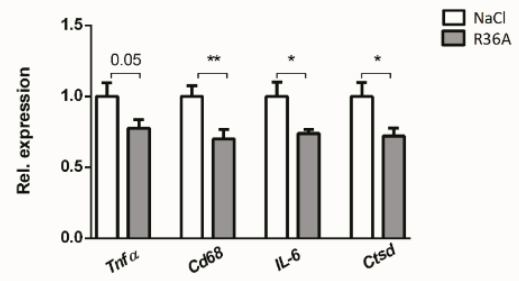

Figure 5: Parameters of spleen inflammation. (A) Hepatic gene expression analysis of Tnf $\alpha, C d 68, I L-6$ and Ctsd. Data are shown relative to $\mathrm{NaCl}$-treated $\mathrm{Npc} 1^{\text {nih }}$ mice. ${ }^{*}$ Indicates $p<0.05$ and ${ }^{* *} p<0.01$ compared to NaCl-treated $N p c 1^{\text {nih }}$ mice by use of two-tailed unpaired $t$ test. $\mathrm{n}=11-12$ mice per group. All error bars are SEM. 


\section{DISCUSSION}

Due to severity of symptoms, delayed diagnosis and restricted therapeutical options, NPC1 disease patients suffer from a gradual impairment in quality of life and premature death. NPC1 disease commonly manifests through systemic symptoms, such as liver and spleen dysfunction, which precede neurological symptoms (epilepsy, movement disorder, dementia, among others). In this study, we show that heat-inactivated pneumococci immunization improves both systemic and neurological symptoms, including motor function, neurodegeneration, neuro- and systemic inflammation in a mouse model of NPC1 disease. In light of these promising results, we propose pneumococcal immunization as a novel therapeutic tool to ameliorate NPC1 disease severity, potentially in combination with treatments such as Miglustat and cyclodextrin $(9,42)$.

Although the role of oxLDL in neurodegenerative diseases has not been extensively investigated, oxLDL is involved in the pathogenesis of NASH and atherosclerosis, two diseases that share pathological mechanisms with NPC1 disease (19, 20, 43-46). Currently, there is no definite consensus on the benefits of targeting oxLDL by increasing anti-oxLDL antibodies in atherosclerosis, as some studies suggest that this approach increases inflammation and exacerbates disease severity (47-49). However, it should be noted that, in these studies, no distinction was made between classes and idiotypes of anti-oxLDL antibodies. Although a study from Smook et al (50) found no correlation between anti-oxLDL IgM autoantibody levels and advanced atherosclerotic lesion formation, this study could not establish whether anti-oxLDL IgM antibodies are anti or pro-atherogenic, and several other in vivo reports have found increased anti-oxLDL IgM antibody levels to be beneficial $(51,52)$. Thus, it is likely that the reported pathogenic effects of anti-oxLDL antibodies are due to the production of anti-oxLDL IgG, rather than IgM, antibodies. In atherosclerosis and NASH, pneumococcal immunization has been shown to ameliorate inflammation and disease severity $(18,20,31)$, suggesting that this treatment may be beneficial to the systemic components of NPC1.

Although no effects were observed on splenic lipid accumulation following pneumococcal immunization, expression of several inflammatory genes was improved in the spleen of immunized Npc1 ${ }^{\text {ih }}$ mice. Of note, in this study, we observed reduced spleen weight in control-treated $N p c 1^{\text {nih }}$ mice compared to wildtype mice instead of characteristic splenomegaly of NPC1 disease, indicating that this model does not recapitulate the spleen NPC1 disease phenotype. In contrast to spleen observations, liver weight and cholesterol accumulation, as well as hepatic inflammation were increased in control-treated $N p c 1^{\text {nih }}$ mice compared to wildtype counterparts. Following pneumococcal immunization, liver apoptosis, 
hepatosplenic inflammation and hepatic cholesterol accumulation were decreased in immunized $N p c 1^{\text {nih }}$ mice, although the latter effects were of low magnitude. In addition to targeting oxLDL, immunization with heat-inactivated pneumococci is also known to improve clearance of apoptotic cells, due to molecular mimicry between apoptotic cells and $S$. pneumoniae (31). Indeed, after immunization, $N p c 1^{\text {nih }}$ mice displayed lower levels of apoptotic liver cells. Therefore, the beneficial effects of heat-inactivated pneumococci immunization in our study can partly derive from increased clearance of apoptotic cells.

As expected, control-treated $N p c 1^{\text {nih }}$ mice showed motor function deficits as assessed by the pole and rotarod tests, confirming neurological impairments in these mice compared to wildtype mice. In this study, unlike control-treated $N p c 1^{\text {nih }}$ mice, the performance of immunized $N p c 1^{\text {nih }}$ mice on the pole test remained unchanged over time. Additionally, immunized $N p c 1^{\text {nih }}$ mice displayed less prominent decay on rotarod test performance compared to their control-treated counterparts. It should be noted that, unlike the beam walking or the catwalk test, several neuronal circuits are involved in pole and rotarod test performance (53). As such, we are unable to pinpoint the specific skills that were improved by pneumococcal immunization. Nonetheless, our results indicate an overall improvement in motor function decay following pneumococcal immunization in $N p c 1^{\text {nih }}$ mice, in line with observed reduction in neuroinflammation and cerebellar damage.

The mechanisms leading to improved neurological features in $N p c 1^{\text {nih }}$ mice following pneumococcal immunization are unclear. IgM antibodies are large molecules and are thus usually precluded from the nervous system due to the blood brain barrier (BBB). As such, whether peripheral immunization strategies can be effective in the nervous system is a controversial matter. Nonetheless, peripheral immunization in Alzheimer's disease and amyotrophic lateral sclerosis has been shown to elicit beneficial effects in the nervous system with as little as $0.1 \%$ antibody passage through the BBB $(54,55)$. Furthermore, it has been proposed that antibodies in the nervous system facilitate the corresponding ligand efflux to the periphery, thus contributing to its clearance (55). As such, it is possible that the neurological improvements observed in this study derive from anti-oxLDL IgM autoantibody-mediated oxLDL clearance to the periphery and/or prevention of oxLDL uptake by microglia scavenger receptors, as has been proposed for macrophages $(56,57)$. On the other hand, live S. pneumoniae can cross the BBB due to certain membrane-surface proteins (58) that may be retained after heat-inactivating the bacteria. Although more thorough analyses are required to derive conclusions regarding microglia activation state, following pneumococcal immunization we observed a shift in microglia morphology that is commonly associated with a phenotypical change in these cells (59). While we are unable to pinpoint whether this is a response to interaction of microglia with anti-oxLDL IgM autoantibodies, 
complement activation or heat-inactivated pneumococci recognition, the latter option is unlikely, as previous reports suggest that heat-inactivated $S$. pneumoniae are nonimmunogenic in the nervous system (60). Of note, in vitro and in vivo studies have shown that endothelial cells in the BBB are susceptible to oxLDL-induced cytotoxicity (61-63). Therefore, it is possible that high levels of plasma oxidized cholesterol products and oxLDL in NPC1 disease increase BBB permeability and exacerbate neurological symptoms, a concept that has previously been proposed in Alzheimer's disease (63). In support of these observations, the observed immune cells in the cortex of untreated $N p c 1^{\text {nih }}$ mice may result from a compromised BBB integrity. As such, improved neurologic symptoms following immunization in NPC1 disease could further derive from an improved BBB phenotype and function.

Persistent systemic- and neuroinflammation are well-described characteristics of NPC1 disease. While, under physiological conditions, inflammatory processes encourage the clearance of toxic compounds and the repair of injured tissues, the inability to overcome a pathological stimulus causes the persistent inflammatory response to become detrimental. Therefore, reducing inflammation and its associated pathological burden might be a promising approach to reduce NPC1 disease severity. In line with this view, administering non-steroidal anti-inflammatory drugs (NSAID) has been shown to improve motor function, body weight and even survival in a mouse model for NPC1 disease (64). Based on these findings, one can speculate on the direct role of inflammation in NPC1 disease severity and progression, despite it not being the primary cause of the disease. Similarly, though also initially not acknowledged as an essential disease component, inflammation is now accepted as a key component in disorders such as atherosclerosis (65) and Alzheimer's disease (66). Therefore, the role of inflammation in NPC1 disease and its usefulness as a therapeutical target should be further investigated.

Currently, therapeutic interventions for NPC1 disease focus on symptom management, and no curative treatments are available (67). As several mechanisms downstream of lysosomal lipid accumulation contribute to NPC1 disease pathology, interventions targeting different key features of NPC1 disease are likely to have increased benefits compared to monotherapeutic startegies. In line with this rationale, in vitro and in vivo studies have analysed the effects of combining therapies to improve cellular lipid accumulation, oxidative stress, inflammation and intracellular calcium homeostasis $(64,68)$. Overall, such studies found that combined therapies outperform the use of single compounds, highlighting the benefits of tackling different pathological mechanisms in NPC1 disease. For instance, the effects of improved cellular lipid accumulation, considered to be at the root of NPC1 disease pathology, are amplified when combined with anti-inflammatory and antioxidant compounds (68). As such, the investigation of pneumococcal immunization in combination with direct lysosomal 
lipid mobilizers (i.e. cyclodextrin) may be an attractive future approach for NPC1 disease patients.

Despite the promising features of pneumococcal vaccination, some points should be addressed before it can be used as a treatment for NPC1 disease. Although the impact of spleen damage on the immune system of NPC1 disease patients has yet to be analysed, it is likely that the latter is compromised to some extent, which may in turn decrease the efficacy of immunotherapy. Furthermore, it was not yet assessed whether pneumococcal immunization is efficient in the context of NPC1 disease not just as a treatment but also as a preventive tool. In addition, in this study, $N p c 1^{\text {nith }}$ mice received two booster pneumococci immunizations, whereas pneumococcal vaccination in humans is usually performed once every several years, which may not be enough for therapeutically relevant oxLDL clearance (69). Nevertheless, it is worth taking into consideration that one of the most common manifestations of NPC1 is dysphagia, whose complications range from malnutrition to lung infections, mainly, bronchopneumonia (70-73). The fact that bronchopneumonia is a major cause of death in NPC1 disease patients is therefore another compelling argument for using pneumococcal immunization in these patients. The use of pneumococcal vaccinations against pneumonia is widespread (74-78). Furthermore, due to its long practice in the clinic, many optimizations of the vaccine have taken place, guaranteeing its safety even in vulnerable individuals such as pregnant women, young children and the elderly (75-77). It should be noted that different S. pneumoniae strains, as well as other bacteria, possess the phosphorylcholine epitope that triggers the production of anti-oxLDL IgM autoantibodies by means of molecular mimicry $(31,79)$. Nevertheless, it was so far not investigated whether immunization using these bacteria also elicits beneficial effects on lipid metabolism and inflammation. Furthermore, in this study, some of the phenotypical improvements, although statistically significant, were of low magnitude (i.e. hepatic cholesterol accumulation). Whether these effects can be enhanced by combining pneumococcal immunization with other NPC1 disease treatments or by using different immunogens should be explored in the future. Overall, considering the promising results in this study, further research is warranted to fully assess the most efficient way to modulate anti-oxLDL immune responses via pneumococcal vaccination in NPC1 disease patients.

\section{Final remarks}

For the first time, we demonstrate that increasing anti-oxLDL IgM autoantibodies ameliorates motor function decay, neuro- and hepatic inflammation, as well as hepatic cholesterol metabolism in NPC1 disease. In light of the present findings, we propose pneumococcal immunization, potentially in combination with other available treatments, as a novel therapeutic tool for NPC1 disease. 


\section{REFERENCES}

1. Patterson MC, Mengel E, Wijburg FA, Muller A, Schwierin B, Drevon H, et al. Disease and patient characteristics in NP-C patients: findings from an international disease registry. Orphanet J Rare Dis. 2013;8:12.

2. Patterson MC, Hendriksz CJ, Walterfang M, Sedel F, Vanier MT, Wijburg F, et al. Recommendations for the diagnosis and management of Niemann-Pick disease type C: an update. Mol Genet Metab. 2012;106(3):330-44.

3. Pfeffer SR. Clues to NPC1-mediated cholesterol export from lysosomes. Proc Natl Acad Sci U S A. 2016;113(29):7941-3.

4. Kelly DA, Portmann B, Mowat AP, Sherlock S, Lake BD. Niemann-Pick disease type $\mathrm{C}$ : diagnosis and outcome in children, with particular reference to liver disease. J Pediatr. 1993;123(2):242-7.

5. Garver WS, Francis GA, Jelinek D, Shepherd G, Flynn J, Castro G, et al. The National Niemann-Pick $\mathrm{C} 1$ disease database: report of clinical features and health problems. Am J Med Genet A. 2007;143A(11):1204-11.

6. Zhang H, Wang Y, Lin N, Yang R, Qiu W, Han L, et al. Diagnosis of Niemann-Pick disease type $\mathrm{C}$ with 7-ketocholesterol screening followed by NPC1/NPC2 gene mutation confirmation in Chinese patients. Orphanet J Rare Dis. 2014;9:82.

7. Greenberg CR, Barnes JG, Kogan S, Seargeant LE. A rare case of Niemann-Pick disease type $C$ without neurological involvement in a 66-year-old patient. Mol Genet Metab Rep. 2015;3:18-20.

8. Sevin M, Lesca G, Baumann N, Millat G, Lyon-Caen O, Vanier MT, et al. The adult form of Niemann-Pick disease type C. Brain. 2007;130(Pt 1):120-33.

9. Ory DS, Ottinger EA, Farhat NY, King KA, Jiang X, Weissfeld L, et al. Intrathecal 2hydroxypropyl-beta-cyclodextrin decreases neurological disease progression in NiemannPick disease, type C1: a non-randomised, open-label, phase 1-2 trial. Lancet. 2017;390(10104):1758-68.

10. Alam MS, Getz M, Haldar K. Chronic administration of an HDAC inhibitor treats both neurological and systemic Niemann-Pick type $C$ disease in a mouse model. Sci Transl Med. 2016;8(326):326ra23.

11. Fu R, Yanjanin NM, Bianconi S, Pavan WJ, Porter FD. Oxidative stress in NiemannPick disease, type C. Mol Genet Metab. 2010;101(2-3):214-8.

12. Rimkunas VM, Graham MJ, Crooke RM, Liscum L. TNF-\{alpha\} plays a role in hepatocyte apoptosis in Niemann-Pick type C liver disease. J Lipid Res. 2009;50(2):327-33.

13. Alam MS, Getz M, Yi S, Kurkewich J, Safeukui I, Haldar K. Plasma signature of neurological disease in the monogenetic disorder Niemann-Pick Type C. J Biol Chem. 2014;289(12):8051-66. 
14. Sayre NL, Rimkunas VM, Graham MJ, Crooke RM, Liscum L. Recovery from liver disease in a Niemann-Pick type C mouse model. J Lipid Res. 2010;51(8):2372-83.

15. Tabas I, Garcia-Cardena G, Owens GK. Recent insights into the cellular biology of atherosclerosis. J Cell Biol. 2015;209(1):13-22.

16. Musso G, Cassader M, Gambino R. Non-alcoholic steatohepatitis: emerging molecular targets and therapeutic strategies. Nat Rev Drug Discov. 2016;15(4):249-74.

17. Hendrikx T, Walenbergh SM, Hofker MH, Shiri-Sverdlov R. Lysosomal cholesterol accumulation: driver on the road to inflammation during atherosclerosis and non-alcoholic steatohepatitis. Obes Rev. 2014;15(5):424-33.

18. Bieghs V, van Gorp PJ, Walenbergh SM, Gijbels MJ, Verheyen F, Buurman WA, et al. Specific immunization strategies against oxidized low-density lipoprotein: a novel way to reduce nonalcoholic steatohepatitis in mice. Hepatology. 2012;56(3):894-903.

19. Houben T, Oligschlaeger Y, Bitorina AV, Hendrikx T, Walenbergh SMA, Lenders M$\mathrm{H}$, et al. Blood-derived macrophages prone to accumulate lysosomal lipids trigger oxLDLdependent murine hepatic inflammation. Scientific Reports. 2017;7(1):12550.

20. Jeurissen MLJ, Walenbergh SMA, Houben T, Gijbels MJJ, Li J, Hendrikx T, et al. Prevention of oxLDL uptake leads to decreased atherosclerosis in hematopoietic NPC1deficient Ldlr-/- mice. Atherosclerosis. 2016;255:59-65.

21. Newman JW, Morisseau C, Hammock BD. Epoxide hydrolases: their roles and interactions with lipid metabolism. Prog Lipid Res. 2005;44(1):1-51.

22. Bjorkhem I, Diczfalusy U. Oxysterols: friends, foes, or just fellow passengers? Arterioscler Thromb Vasc Biol. 2002;22(5):734-42.

23. Porter FD, Scherrer DE, Lanier MH, Langmade SJ, Molugu V, Gale SE, et al. Cholesterol oxidation products are sensitive and specific blood-based biomarkers for Niemann-Pick C1 disease. Sci Transl Med. 2010;2(56):56ra81.

24. Jiang X, Sidhu R, Porter FD, Yanjanin NM, Speak AO, te Vruchte DT, et al. A sensitive and specific LC-MS/MS method for rapid diagnosis of Niemann-Pick C1 disease from human plasma. J Lipid Res. 2011;52(7):1435-45.

25. Loftus SK, Morris JA, Carstea ED, Gu JZ, Cummings C, Brown A, et al. Murine Model of Niemann-Pick C Disease: Mutation in a Cholesterol Homeostasis Gene. Science. 1997;277(5323):232.

26. Morris MD, Bhuvaneswaran C, Shio H, Fowler S. Lysosome lipid storage disorder in NCTR-BALB/c mice. I. Description of the disease and genetics. The American journal of pathology. 1982;108(2):140-9.

27. Shio H, Fowler S, Bhuvaneswaran C, Morris MD. Lysosome lipid storage disorder in NCTR-BALB/c mice. II. Morphologic and cytochemical studies. The American journal of pathology. 1982;108(2):150-9. 
28. Bhuvaneswaran C, Morris MD, Shio H, Fowler S. Lysosome lipid storage disorder in NCTR-BALB/c mice. III. Isolation and analysis of storage inclusions from liver. The American journal of pathology. 1982;108(2):160-70.

29. Reid PC, Sugii S, Chang T-Y. Trafficking defects in endogenously synthesized cholesterol in fibroblasts, macrophages, hepatocytes, and glial cells from Niemann-Pick type C1 mice. Journal of Lipid Research. 2003;44(5):1010-9.

30. Hovakimyan M, Maass F, Petersen J, Holzmann C, Witt M, Lukas J, et al. Combined therapy with cyclodextrin/allopregnanolone and miglustat improves motor but not cognitive functions in Niemann-Pick Type C1 mice. Neuroscience. 2013;252:201-11.

31. Binder CJ, Horkko S, Dewan A, Chang MK, Kieu EP, Goodyear CS, et al. Pneumococcal vaccination decreases atherosclerotic lesion formation: molecular mimicry between Streptococcus pneumoniae and oxidized LDL. Nat Med. 2003;9(6):736-43.

32. Briles DE, Forman C, Hudak S, Claflin JL. Anti-phosphorylcholine antibodies of the T15 idiotype are optimally protective against Streptococcus pneumoniae. J Exp Med. 1982;156(4):1177-85.

33. Bieghs V, Wouters K, van Gorp PJ, Gijbels MJ, de Winther MP, Binder CJ, et al. Role of scavenger receptor A and CD36 in diet-induced nonalcoholic steatohepatitis in hyperlipidemic mice. Gastroenterology. 2010;138(7):2477-86, 86 e1-3.

34. Bieghs V, Verheyen F, van Gorp PJ, Hendrikx T, Wouters K, Lutjohann D, et al. Internalization of modified lipids by CD36 and SR-A leads to hepatic inflammation and lysosomal cholesterol storage in Kupffer cells. PLoS One. 2012;7(3):e34378.

35. Pelsers MM, Butler PJ, Bishop CM, Glatz JF. Fatty acid binding protein in heart and skeletal muscles of the migratory barnacle goose throughout development. Am J Physiol. 1999;276(3 Pt 2):R637-43.

36. Loncarevic-Vasiljkovic N, Pesic V, Todorovic S, Popic J, Smiljanic K, Milanovic D, et al. Caloric Restriction Suppresses Microglial Activation and Prevents Neuroapoptosis Following Cortical Injury in Rats. PLOS ONE. 2012;7(5):e37215.

37. Ogawa N, Hirose Y, Ohara S, Ono T, Watanabe Y. A simple quantitative bradykinesia test in MPTP-treated mice. Res Commun Chem Pathol Pharmacol. 1985;50(3):435-41.

38. Matsuura K, Kabuto H, Makino H, Ogawa N. Pole test is a useful method for evaluating the mouse movement disorder caused by striatal dopamine depletion. J Neurosci Methods. 1997;73(1):45-8.

39. German DC, Quintero EM, Liang C, Xie C, Dietschy JM. Degeneration of neurons and glia in the Niemann-Pick $C$ mouse is unrelated to the low-density lipoprotein receptor. Neuroscience. 2001;105(4):999-1005. 
40. Marques AR, Gabriel TL, Aten J, van Roomen CP, Ottenhoff R, Claessen N, et al. Gpnmb Is a Potential Marker for the Visceral Pathology in Niemann-Pick Type C Disease. PLoS One. 2016;11(1):e0147208.

41. Zhan SS, Jiang JX, Wu J, Halsted C, Friedman SL, Zern MA, et al. Phagocytosis of apoptotic bodies by hepatic stellate cells induces NADPH oxidase and is associated with liver fibrosis in vivo. Hepatology. 2006;43(3):435-43.

42. Wraith JE, Imrie J. New therapies in the management of Niemann-Pick type C disease: clinical utility of miglustat. Therapeutics and clinical risk management. 2009;5:87787.

43. Hörkkö S, Binder CJ, Shaw PX, Chang M-K, Silverman G, Palinski W, et al. Immunological responses to oxidized LDL. Free Radical Biology and Medicine. 2000;28(12):1771-9.

44. Colles SM, Maxson JM, Carlson SG, Chisolm GM. Oxidized LDL-Induced Injury and Apoptosis in Atherosclerosis: Potential Roles for Oxysterols. Trends in Cardiovascular Medicine. 2001;11(3):131-8.

45. Praticò D. Lipid Peroxidation in Mouse Models of Atherosclerosis. Trends in Cardiovascular Medicine. 2001;11(3):112-6.

46. Palinski W, Witztum JL. Immune responses to oxidative neoepitopes on LDL and phospholipids modulate the development of atherosclerosis. Journal of Internal Medicine. 2001;247(3):371-80.

47. Shoenfeld Y, Wu R, Dearing Linda D, Matsuura E. Are Anti-Oxidized Low-Density Lipoprotein Antibodies Pathogenic or Protective? Circulation. 2004;110(17):2552-8.

48. Zhang J, Wang D, He S. Roles of antibody against oxygenized low density lipoprotein in atherosclerosis: recent advances. International journal of clinical and experimental medicine. 2015;8(8):11922-9.

49. Lopes-Virella MF, Virella G. Clinical significance of the humoral immune response to modified LDL. Clinical Immunology. 2010;134(1):55-65.

50. Smook MLF, van Leeuwen M, Heeringa P, Damoiseaux JGMC, Theunissen R, Daemen MJAP, et al. Anti-oxLDL antibody isotype levels, as potential markers for progressive atherosclerosis in APOE and APOECD40L mice. Clinical and experimental immunology. 2008;154(2):264-9.

51. Hosseini H, Li Y, Kanellakis P, Tay C, Cao A, Tipping P, et al. Phosphatidylserine liposomes mimic apoptotic cells to attenuate atherosclerosis by expanding polyreactive IgM producing B1a lymphocytes. Cardiovascular Research. 2015;106(3):443-52.

52. Shaw PX, Hörkkö S, Chang M-K, Curtiss LK, Palinski W, Silverman GJ, et al. Natural antibodies with the T15 idiotype may act in atherosclerosis, apoptotic clearance, and protective immunity. The Journal of Clinical Investigation. 2000;105(12):1731-40. 
53. Mann A, Chesselet M-F. Chapter 8 - Techniques for Motor Assessment in Rodents. In: LeDoux MS, editor. Movement Disorders (Second Edition). Boston: Academic Press; 2015. p. 139-57.

54. Banks WA, Terrell B, Farr SA, Robinson SM, Nonaka N, Morley JE. Passage of amyloid $\beta$ protein antibody across the blood-brain barrier in a mouse model of Alzheimer's disease. Peptides. 2002;23(12):2223-6.

55. $\mathrm{Xu}$ X, Denic A, Jordan LR, Wittenberg NJ, Warrington AE, Wootla B, et al. A natural human IgM that binds to gangliosides is therapeutic in murine models of amyotrophic lateral sclerosis. Disease Models \&amp;amp; Mechanisms. 2015;8(8):831.

56. Wilkinson K, El Khoury J. Microglial scavenger receptors and their roles in the pathogenesis of Alzheimer's disease. International journal of Alzheimer's disease. 2012;2012:489456-.

57. Hörkkö S, Bird DA, Miller E, Itabe H, Leitinger N, Subbanagounder G, et al. Monoclonal autoantibodies specific for oxidized phospholipids or oxidized phospholipidprotein adducts inhibit macrophage uptake of oxidized low-density lipoproteins. The Journal of clinical investigation. 1999;103(1):117-28.

58. Coureuil M, Lécuyer H, Bourdoulous S, Nassif X. A journey into the brain: insight into how bacterial pathogens cross blood-brain barriers. Nature Reviews Microbiology. 2017;15:149.

59. Fernández-Arjona MdM, Grondona JM, Granados-Durán P, Fernández-Llebrez P, López-Ávalos MD. Microglia Morphological Categorization in a Rat Model of Neuroinflammation by Hierarchical Cluster and Principal Components Analysis. Frontiers in Cellular Neuroscience. 2017;11(235).

60. Mook-Kanamori BB, Geldhoff M, van der Poll T, van de Beek D. Pathogenesis and pathophysiology of pneumococcal meningitis. Clinical microbiology reviews. 2011;24(3):55791.

61. Lin Y-L, Chang H-C, Chen T-L, Chang J-H, Chiu W-T, Lin J-W, et al. Resveratrol Protects against Oxidized LDL-Induced Breakage of the Blood-Brain Barrier by Lessening Disruption of Tight Junctions and Apoptotic Insults to Mouse Cerebrovascular Endothelial Cells. The Journal of Nutrition. 2010;140(12):2187-92.

62. Schreurs MPH, Cipolla MJ. Cerebrovascular dysfunction and blood-brain barrier permeability induced by oxidized LDL are prevented by apocynin and magnesium sulfate in female rats. Journal of cardiovascular pharmacology. 2014;63(1):33-9.

63. Dias Irundika HK, Polidori Maria C, Griffiths Helen R. Hypercholesterolaemiainduced oxidative stress at the blood-brain barrier. Biochemical Society Transactions. 2014;42(4):1001. 
64. Smith D, Wallom K-L, Williams IM, Jeyakumar M, Platt FM. Beneficial effects of antiinflammatory therapy in a mouse model of Niemann-Pick disease type C1. Neurobiology of Disease. 2009;36(2):242-51.

65. Libby P. Inflammation in Atherosclerosis. Arteriosclerosis, Thrombosis, and Vascular Biology. 2012;32(9):2045.

66. McGeer PL, McGeer EG. The amyloid cascade-inflammatory hypothesis of Alzheimer disease: implications for therapy. Acta Neuropathologica. 2013;126(4):479-97.

67. Madra M, Sturley SL. Niemann-Pick type C pathogenesis and treatment: from statins to sugars. Clinical lipidology. 2010;5(3):387-95.

68. Williams IM, Wallom K-L, Smith DA, Al Eisa N, Smith C, Platt FM. Improved neuroprotection using miglustat, curcumin and ibuprofen as a triple combination therapy in Niemann-Pick disease type C1 mice. Neurobiology of Disease. 2014;67:9-17.

69. Hurley LP, Allison MA, Pilishvili T, O'Leary ST, Crane LA, Brtnikova M, et al. Primary Care Physicians' Struggle with Current Adult Pneumococcal Vaccine Recommendations. The Journal of the American Board of Family Medicine. 2018;31(1):94-104. 70. Wraith JE, Guffon N, Rohrbach M, Hwu WL, Korenke GC, Bembi B, et al. Natural history of Niemann-Pick disease type $\mathrm{C}$ in a multicentre observational retrospective cohort study. Molecular Genetics and Metabolism.98(3):250-4.

71. Iturriaga C, Pineda M, Fernández-Valero EM, Vanier MT, Coll MJ. Niemann\&\#x2013;Pick C disease in Spain: Clinical spectrum and development of a disability scale. Journal of the Neurological Sciences.249(1):1-6.

72. Wraith JE, Baumgartner MR, Bembi B, Covanis A, Levade T, Mengel E, et al. Recommendations on the diagnosis and management of Niemann-Pick disease type C. Molecular Genetics and Metabolism.98(1):152-65.

73. Walterfang M, Chien Y-H, Imrie J, Rushton D, Schubiger D, Patterson MC. Dysphagia as a risk factor for mortality in Niemann-Pick disease type C: systematic literature review and evidence from studies with miglustat. Orphanet Journal of Rare Diseases. 2012;7(1):76.

74. Jackson LA, Gurtman A, van Cleeff M, Jansen KU, Jayawardene D, Devlin C, et al. Immunogenicity and safety of a 13-valent pneumococcal conjugate vaccine compared to a 23valent pneumococcal polysaccharide vaccine in pneumococcal vaccine-naive adults. Vaccine. 2013;31(35):3577-84.

75. Pomat WS, van den Biggelaar AHJ, Phuanukoonnon S, Francis J, Jacoby P, Siba PM, et al. Safety and Immunogenicity of Neonatal Pneumococcal Conjugate Vaccination in Papua New Guinean Children: A Randomised Controlled Trial. PLOS ONE. 2013;8(2):e56698.

76. Bryant KA, Block SL, Baker SA, Gruber WC, Scott DA. Safety and Immunogenicity of a 13-Valent Pneumococcal Conjugate Vaccine. Pediatrics. 2010;125(5):866. 
77. Ciprero K, Zykov KA, Briko NI, Shekar T, Sterling TM, Bitieva E, et al. Safety and immunogenicity of a single dose 23-valent pneumococcal polysaccharide vaccine in Russian subjects. Human Vaccines \& Immunotherapeutics. 2016;12(8):2142-7.

78. Namkoong H, Funatsu Y, Oishi K, Akeda Y, Hiraoka R, Takeshita K, et al. Comparison of the immunogenicity and safety of polysaccharide and protein-conjugated pneumococcal vaccines among the elderly aged 80 years or older in Japan: An open-labeled randomized study. Vaccine. 2015;33(2):327-32.

79. Kolberg J, Høiby EA, Jantzen E. Detection of the phosphorylcholine epitope in streptococci,Haemophilusand pathogenicNeisseriaeby immunoblotting. Microbial Pathogenesis. 1997;22(6):321-9. 


\section{SUPPLEMENTARY MATERIALS}

S1
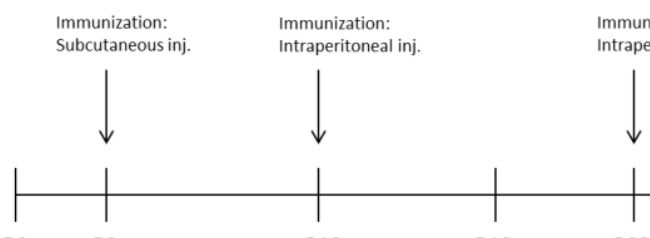

D0 D3

D10

2 week-old

Npc1 $1^{\text {nih }}$ mice

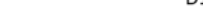

Supplementary figure S1: Overview of experimental set-up.

\section{S2}

Type 1

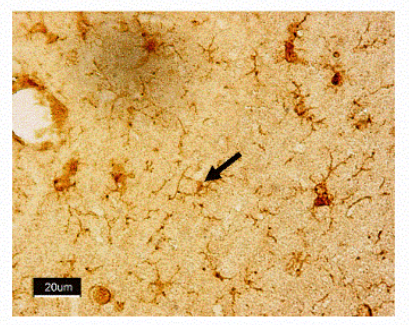

Type 2

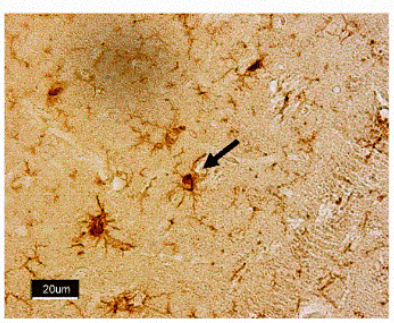

Type 3

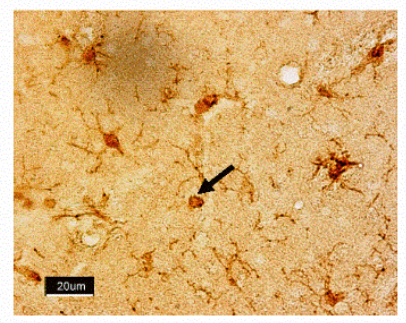

Supplementary figure S2: Representative pictures of microglia phenotype scoring (400x). 
S3A

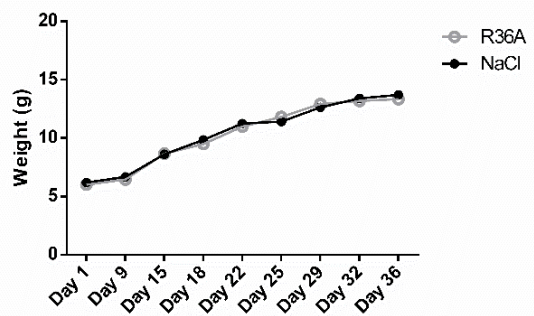

S3B

S3C
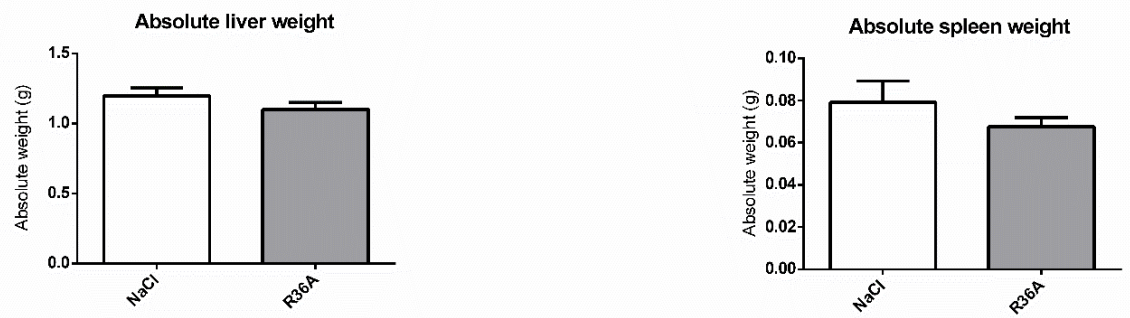

Supplementary figure S3: General parameters. (S3A) Weight of $N p c 1^{\text {nih }}$ mice throughout study. (S3B -S3C) Absolute liver and spleen weight. 
S4

Brain

\begin{tabular}{c|l|l|} 
Motor function & \multicolumn{2}{c}{ Spearmann correlation } \\
\cline { 2 - 3 } Pole test day 32 & $\mathrm{P}=0.0459$ & $\mathrm{P}=0.0075$ \\
$\mathrm{R}=-0.4297$ & $\mathrm{R}=-0.5539$ \\
\cline { 2 - 3 } Rota rod day 33 & $\mathrm{P}=0.9804$ & $\mathrm{P}=0.7985$ \\
$\mathrm{R}=0.005436$ & $\mathrm{R}=0.05634$ \\
\hline
\end{tabular}

Cerebrum

\begin{tabular}{c|l|l|}
\cline { 2 - 3 } Noc2 & $\mathrm{P}=0.076$ & $\mathrm{P}=0.0857$ \\
& $\mathrm{R}=-0.633$ & $\mathrm{R}=-0.6167$ \\
\cline { 2 - 3 } Ctsd & $\mathrm{P}=0.1206$ & $\mathrm{P}=0.1618$ \\
$\mathrm{R}=-0.5667$ & $\mathrm{R}=0.5167$ \\
\cline { 2 - 3 } Cd68 & $\mathrm{P}=0.0234$ & $\mathrm{P}=0.0126$ \\
$\mathrm{R}=-0.7212$ & $\mathrm{R}=0.7697$ \\
\cline { 2 - 3 } C $/ 3$ & $\mathrm{P}=0.1334$ & $\mathrm{P}=0.0734$ \\
& $\mathrm{R}=0.5152$ & $\mathrm{R}=0.600$ \\
\cline { 2 - 3 } Cxc/10 & $\mathrm{P}=0.1440$ & $\mathrm{P}=0.0734$ \\
$\mathrm{R}=0.5030$ & $\mathrm{R}=0.6000$ \\
\cline { 2 - 3 } & &
\end{tabular}

Cerebellum

\begin{tabular}{|c|c|c|}
\hline Npc2 & $\begin{array}{l}P=0.0968 \\
R=-0.600\end{array}$ & $\begin{array}{l}P=0.0369 \\
R=-0.7167\end{array}$ \\
\hline Ctsd & $\begin{array}{l}P=0.4684 \\
R=-0.2455\end{array}$ & $\begin{array}{l}P=0.6337 \\
R=-0.1636\end{array}$ \\
\hline Cd68 & $\begin{array}{l}P=0.1049 \\
R=-0.5515\end{array}$ & $\begin{array}{l}\mathrm{P}=0.2325 \\
\mathrm{R}=-0.4182\end{array}$ \\
\hline $\mathrm{Cc} / 3$ & $\begin{array}{l}P=0.0806 \\
R=-0.5879\end{array}$ & $\begin{array}{l}P=0.1231 \\
R=-0.5273\end{array}$ \\
\hline $\operatorname{cxc} 110$ & $\begin{array}{l}P=0.1663 \\
R=-0.4788\end{array}$ & $\begin{array}{l}P=0.2632 \\
R=-0.3939\end{array}$ \\
\hline
\end{tabular}

Liver

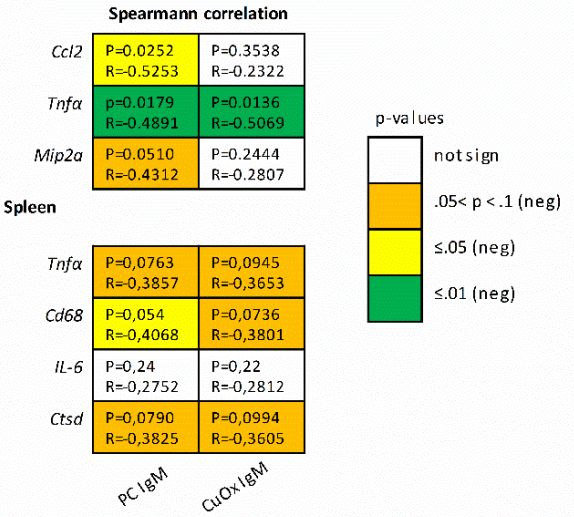

Supplementary figure S4: Correlations between IgM antibody levels and gene expression levels of markers in the brain, liver and spleen. 
S5A

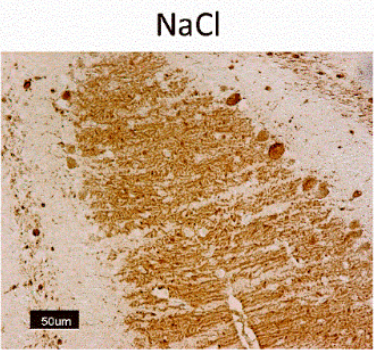

R36A

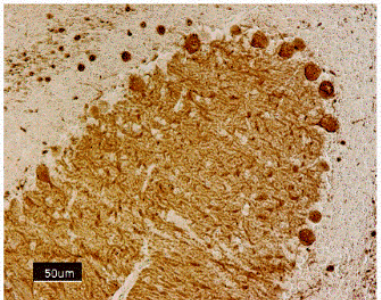

S5B

$\mathrm{NaCl}$
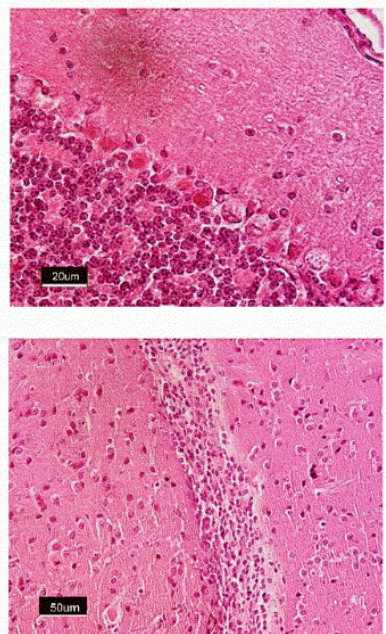

R36A
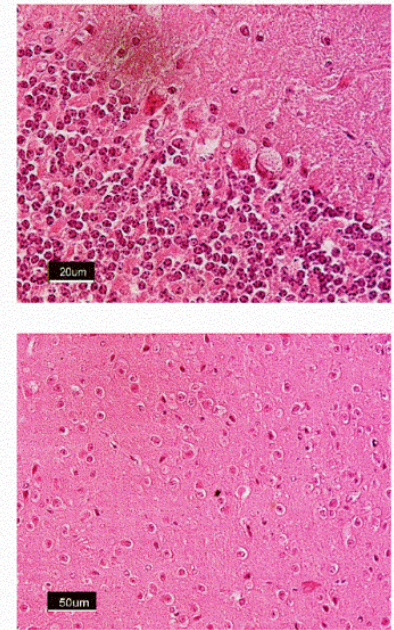

Supplementary figure S5: Representative pictures and scoring of calbindin staining of Purkinje cells in cerebellum (400x) (S5A). General histology of the brain by HE staining: Purkinje cells (top panel, 400x) and immune cell (bottom left panel, 200x) (S5B).

S6

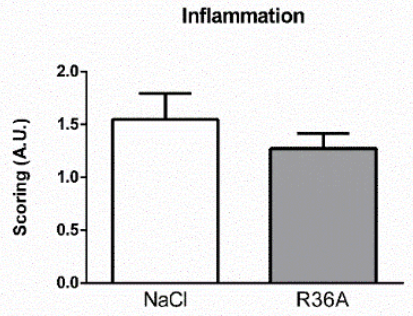

Supplementary figure S6: Liver HE inflammation scoring. 
Supplementary Table S1: Parameters of 7 week-old wildtype and control-treated Npc1 ${ }^{\text {nih }}$ mice

\begin{tabular}{|c|c|c|c|}
\hline & $W t$ & $N p c 1^{\text {nih }}$ & p value \\
\hline \multicolumn{4}{|l|}{ Body parameters } \\
\hline Body weight & $17.68 \pm 0.56$ & $13.69 \pm 0.51$ & $<0.0001$ \\
\hline Liver weight & $0.93 \pm 0.04$ & $1.20 \pm 0.06$ & 0.002 \\
\hline Spleen weight & $0.17 \pm 0.01$ & $0.08 \pm 0.01$ & $<0.0001$ \\
\hline \multicolumn{4}{|l|}{ Motor function } \\
\hline \multicolumn{4}{|l|}{ Pole test } \\
\hline t1 & $5.60 \pm 0.36$ & $9.28 \pm 0.56$ & $<0.0001$ \\
\hline t2 & $5.89 \pm 0.75$ & $8.91 \pm 0.53$ & 0.003 \\
\hline t3 & $7.18 \pm 0.76$ & $13.96 \pm 2.38$ & 0.02 \\
\hline \multicolumn{4}{|l|}{ Rotarod } \\
\hline t1 & $267.60 \pm 10.83$ & $231.0 \pm 9.25$ & 0.02 \\
\hline t2 & $245.7 \pm 15.59$ & $180.9 \pm 10.94$ & 0.003 \\
\hline \multicolumn{4}{|l|}{ Histology } \\
\hline $\begin{array}{l}\text { Infiltrating macrophages and } \\
\text { neutrophils }(\mathrm{Mac1}) \\
\left.\text { positive cells } / \mathrm{mm}^{2}\right)\end{array}$ & $29.74 \pm 4.67$ & $51.56 \pm 5.64$ & 0.01 \\
\hline \multicolumn{4}{|l|}{ Cholesterol parameters } \\
\hline Plasma cholesterol (mM) & $2.52 \pm 0.11$ & $2.96 \pm 0.12$ & 0.02 \\
\hline $\begin{array}{l}\text { Liver cholesterol } \\
\text { (mg total cholesterol/mg protein) }\end{array}$ & $0.006 \pm 0.0003$ & $0.025 \pm 0.0007$ & $<0.0001$ \\
\hline
\end{tabular}

$p$ value compares wildtype to $N p c 1^{\text {nih }}$ mice by use of two-tailed unpaired $t$ test. Standards of error represent \pm SEM. 



\section{Chapter 6}

The Influence of a Conjugated Pneumococcal Vaccination on Plasma Antibody Levels against Oxidized LowDensity Lipoprotein in Metabolic Disease Patients: A Single-Arm Pilot Clinical Trial.

Ronit Shiri-Sverdlov, Inês Magro dos Reis, Yvonne Oligschlaeger, Tim Hendrikx, Dennis M. Meesters, Annick Vanclooster, Nele Vanhoutvin, Ger H. Koek, Marit Westerterp, Christoph J. Binder, David Cassiman, Tom Houben 


\section{ABSTRACT}

As a mediator between lipid metabolism dysfunction, oxidative stress and inflammation, oxidized low-density lipoprotein (oxLDL) is a promising therapeutical target in a wide range of metabolic diseases. In mice, pneumococcal immunization increases anti-phosphorylcholine and oxLDL antibody levels, and reduces atherosclerosis, non-alcoholic steatohepatitis and Niemann-Pick disease burden. These findings suggest that pneumococcal vaccination may be a useful preventive and therapeutical strategy in metabolic disease patients. In this pilot clinical trial, our aim was to determine whether the administration of a pneumococcal vaccine increases anti-phosphorylcholine and anti-oxLDL antibody levels in metabolic disease patients. The following patients were enrolled: four patients with familial partial lipodystrophy (all women, mean age 32 years old); three familial hypercholesterolemia patients (one girl, two boys; mean age 13 years); and two Niemann-Pick type B (NP-B) patients (two men, mean age 37.5 years old). Participants received one active dose of a 13valent conjugated pneumococcal vaccine (Prevenar 13) and were followed-up for four weeks. Four weeks after Prevenar 13 vaccination, no differences were observed in patients' levels of anti-oxLDL IgM or IgG antibodies. In addition, we observed a reduction in antiphosphorylcholine (anti-PC) IgM antibody levels, whereas no differences were observed in anti-PC IgG antibody titers. These findings indicate that Prevenar 13 vaccination does not induce an immune response against oxLDL in patients with metabolic diseases. Therefore, Prevenar 13 is not suited to target the metabolic disruptor and pro-inflammatory mediator oxLDL in patients. 


\section{INTRODUCTION}

Lipids perform extensive biological functions, including the modulation of cell membrane composition, acting as signaling molecules and contributing to the production and storage of energy. As such, lipid metabolism is a complex and tightly regulated whole-body process, and dysfunctions in lipid metabolism can lead to dire consequences for the organism. The importance of a properly functioning lipid metabolism is illustrated by the disease burden tied to the wide array of known acquired and inherited lipid metabolic diseases. Currently, widespread lifestyle habits, such as unhealthy diets and low physical activity, are major propellers of dyslipidemia and metabolic syndrome (1). In addition, a considerable number of individuals are genetically predisposed to develop metabolic dysfunction, including familial hypercholesterolemia (FH), lipodystrophy (LPD) and Niemann-Pick (NP) disease patients. Regardless of the underlying etiology, patients with lipid metabolic dysfunctions are at high risk of developing non-communicable diseases such as type 2 diabetes, cardiovascular and liver disease and cancer, which are estimated to account for nearly $70 \%$ of premature deaths worldwide (2). As such, the development of preventive and therapeutic strategies targeting disorders of lipid metabolism and associated disease burden is of utmost importance for patients, clinicians and societies alike.

Among other factors, an increase in the production of oxidized low-density lipoprotein (oxLDL) has emerged as an important metabolic disruptor and pro-inflammatory mediator in metabolic diseases and associated non-communicable diseases. OxLDL is broadly categorized as minimally or extensively modified LDL, and a wide range of oxLDL particles have been observed, varying both in chemical structure and biological effects (3). Unlike native LDL, oxLDL binds to scavenger receptors, toll-like receptors and LOX-1 receptors, triggering cytotoxic, pro-inflammatory and apoptotic pathways in the cells $(3,4)$. Although oxLDL has mostly been studied in the context of atherosclerosis and cardiovascular disease, an increasing body of evidence points towards the involvement of oxLDL in metabolic syndrome, type 2 diabetes and cancer $(5,6)$. Altogether, these studies indicate that oxLDL is a link between metabolic dysfunction, oxidative stress and inflammation, and is thus a promising therapeutic target for patients with acquired and inherited lipid metabolic disturbances.

Previously, studies have shown that cardiovascular disease patients with higher anti-oxLDL IgM antibody levels have a reduced atherosclerotic burden and a better prognosis, suggesting that anti-oxLDL antibodies have protective characteristics in atherosclerosis (7-9). Notably, antibodies targeting oxidized lipids and associated products, such as those present in oxLDL, are naturally produced from birth in mice and humans alike, even before the immune system 
encounters such threats (10). These so-called "natural autoantibodies" consist of IgM antibodies that mainly bind to malondialdehyde (MDA) and to phosphorylcholine (PC), a head group commonly present in phospholipids. Whereas in healthy cells and native LDL the PC moiety of phospholipids is unavailable for recognition by the immune system, PC becomes exposed and available for antibody binding in oxidized lipids and apoptotic cells (11). In addition, PC epitopes are found in Streptococcus pneumoniae, a bacterium well known for inducing respiratory and brain disease. As such, anti-PC IgM autoantibodies protect the host against oxidized self-antigens and foreign S. pneumoniae alike, thus acting as a primary line of defense against such pathogenic agents. Conversely, studies have shown that heat-inactivated S. pneumoniae immunization increases anti-oxLDL antibody levels and reduces disease progression and burden in animal models for atherosclerosis, non-alcoholic steatohepatitis and Niemann-Pick type C1 disease (11-14). While the aforementioned diseases differ in etiology and affected tissues and organs, all are characterized by metabolic dysfunction, lysosomal cholesterol accumulation, oxidative stress and inflammation, indicating that anti-oxLDL antibodies may have a broad therapeutic application and may serve as a useful therapeutic strategy in a wide array of metabolic diseases.

In the current pilot study, our aim was to assess whether a pneumococcal vaccine increases anti-oxLDL and anti-PC IgM and/or IgG antibody levels in patients with inherited metabolic diseases. Patients with the following metabolic diseases were eligible for this study: familial partial LPD (OMIM entry \# 151660), FH (OMIM entry \#143890) and NP type B and C (NPB and NPC, OMIM entries \#607616, and \#257220 and \#607625, respectively). Ultimately, four LPD, three FH and two NPB patients participated in this study. LPD is a rare genetic disease which results in abnormal adipose tissue distribution, predisposing patients to develop metabolic syndrome, type 2 diabetes mellitus, and cardiovascular and liver disease. On the other hand, FH patients possess genetic mutations that interfere with cellular uptake of LDL, leading to high blood LDL levels from childhood that put FH patients at risk of developing metabolic syndrome and metabolic syndrome associated diseases. Finally, NPB patients are characterized by deficits in sphingomyelinase activity which lead to lysosomal accumulation of sphingomyelin, cumulating in hepatosplenomegaly and lung disease. In order to determine whether pneumococcal vaccination increases anti-oxLDL and anti-PC IgM and IgG antibodies, patients received one active dose of Prevenar 13, a pneumococcal conjugate vaccine routinely used in the clinic. Plasma antibody levels were measured before and four weeks after vaccination. This study's findings indicate that administration of a single dose of Prevenar 13 does not increase antibody levels against PC or oxLDL in metabolic patients. While studies with larger, more homogenous populations are required to confirm these findings, our study suggests that alternative therapeutical strategies are needed in order to target oxLDL in human patients. 


\section{MATERIALS AND METHODS}

\section{Patient Recruitment and Inclusion}

Participants were recruited at the outpatient clinic for metabolic diseases at Ziekenhuis OostLimburg (Genk, Belgium). Data were collected between April 2018 and March 2019. Patients with NPB and NPC disease, FH and familial partial LPD were eligible to participate in the study. Furthermore, in order to participate in the study (1) patients had to be older than 10 years of age; (2) patients must not present any health conditions that might interfere with the study procedures, including autoimmune diseases, immune deficiency, Hodgkin lymphoma and splenectomy syndrome; (3) patients must not consume alcohol in excess (>20 g/day for men and >10 g/day for women); and (4) patients must not be illiterate.

Eligible patients were informed about the study and invited to participate during regular appointments with their clinician. All study participants, or their legal guardians in the case of underage participants, signed an informed consent prior to the start of the study. The study was approved by the Ethics Committee at Leuven University and was registered under the EU Clinical Trials Register (EudraCT 2015-004846-25) and in the NIH clinical trials database (clinicaltrial.gov, study identifier NCT02707211).

\section{Study Procedure and Outcomes}

Patients were randomly assigned identification codes during each visit, so that posterior sample analysis was blindly performed. On the first visit, fasted venous blood samples were taken, followed by a vaccination with the maximum recommended one-time dose of $0.5 \mathrm{~mL}$ of Prevenar 13 (Pfizer). The last visit took place after four weeks, during which blood samples were once again taken. During each visit, $20 \mathrm{~mL}$ of blood was taken via intravenous injection from each patient and stored in EDTA-coated tubes. Blood samples were kept in ice-filled insulated containers until they reached Maastricht University (Maastricht, the Netherlands) on the same day. At Maastricht University, blood samples were spun at $2700 \mathrm{~g}$ for $10 \mathrm{~min}$ at room temperature, and the resulting plasma was aliquoted and stored at $-80{ }^{\circ} \mathrm{C}$ for posterior analysis. The planned outcome of this pilot study was to assess changes in lipid metabolism and inflammation following Prevenar 13 vaccination and subsequent increase in antioxLDL/PC antibody levels. Since we did not observe an increase in anti-oxLDL IgM antibody levels, our new goal was to assess anti-oxLDL and anti-PC antibody titers before, and four weeks after Prevenar 13 vaccination.

\section{Antibody Measurements}

Plasma levels of IgG and IgM antibodies against Prevenar, oxLDL (copper oxidized moiety) and PC-BSA were measured via chemiluminescent ELISA as previously described (15). 


\section{Statistical Analysis}

Since some of the diseases included in this study are very rare (namely, LPD and NiemannPick type B (NP-B)) and this was a pilot clinical study, no power calculations were performed to calculate sample size prior to the beginning of the study. Primary study outcomes, namely, anti-oxLDL and anti-PC IgM and IgG antibody levels before and after Prevenar 13 vaccination, were measured via two-tailed paired t-test ${ }^{*} \mathrm{p} \leq 0.05 ;{ }^{* *} \mathrm{p}<0.01 ;{ }^{* * *} \mathrm{p}<0.001$; $\left.{ }^{* * * *} \mathrm{p}<0.0001\right)$. All data are expressed as the group mean \pm standard deviation of the mean.

Data were statistically analyzed using GraphPad Prism software (version 6, GraphPad Software Inc., San Diego, CA, USA). 


\section{RESULTS}

Patient Population and Study Outcomes

Between 2017 and 2020, seventeen metabolic disease patients were approached to participate in this study, out of which nine were included (Figure 1). The final study population consisted of four familial partial LPD patients, three FH patients and two NPB patients. An overview of the study population is presented in Table 1.

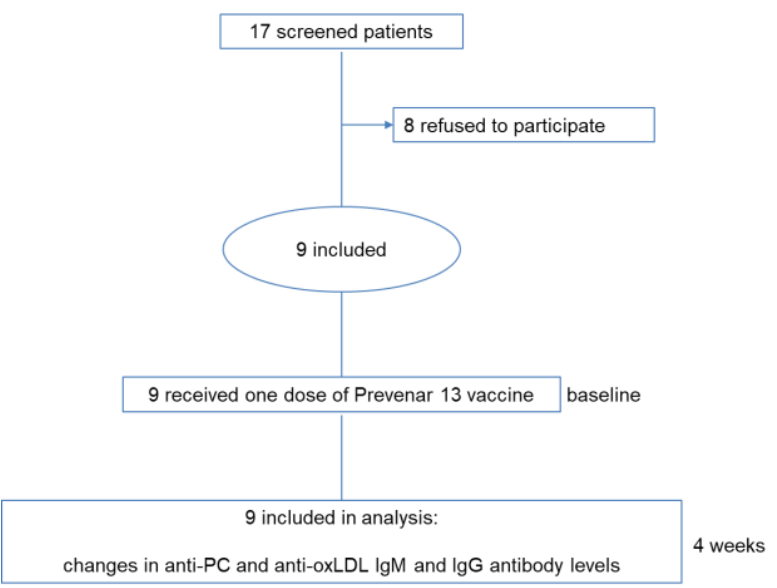

Figure 1. Study design.

Table 1. Characteristics of study population.

\begin{tabular}{l|c|c|}
\hline \multirow{2}{*}{ Disease } & Sex & Age \\
\hline \multirow{4}{*}{ Familial partial lipodystrophy } & Female & 28 \\
\cline { 2 - 3 } & Female & 38 \\
\cline { 2 - 3 } & Female & 37 \\
\cline { 2 - 3 } & Female & 38 \\
\hline \multirow{2}{*}{ Familial hypercholesterolemia } & Male & 15 \\
\cline { 2 - 3 } & Female & 11 \\
\cline { 2 - 3 } & Male & 13 \\
\hline \multirow{2}{*}{ Niemann-Pick type B } & Male & 36 \\
\cline { 2 - 3 } & Male & 38 \\
\hline
\end{tabular}




\section{Prevenar 13 Vaccination Induced an Increase in Prevenar-Specific IgM and IgG Antibodies}

In order to confirm that Prevenar 13 vaccination induced an immune response in patients, Prevenar 13-specific plasma IgM and IgG antibody levels were measured. Four weeks after vaccination, patients displayed increased anti-Prevenar 13 IgM and IgG antibody titers, proving that the vaccine induced an appropriate immune response (Figure 2A,B). To explore potential disease-specific differences within our study population, we further analyzed Prevenar-specific antibody levels among LPD, FH and NPB patients. In LPD patients, Prevenar 13 vaccination induced an increase in anti-Prevenar IgM and IgG antibodies (Figure 2C,D). Likewise, Prevenar 13 vaccination increased Prevenar-specific antibody levels in FH patients. Furthermore, we observed an increase in Prevenar-specific IgG antibody levels in FH patients, although this did not reach statistical significance (Figure 2E,F). While we could not perform statistical analysis on the data from NPB patients $(n=2)$, both anti-Prevenar IgM and IgG antibodies levels tended to increase following vaccination (Figure 2G,H). A complete overview of the descriptive statistical analyses is depicted in Table S1. Overall, these findings indicate that Prevenar 13 vaccination successfully elicited an immune response.

\section{Prevenar 13 Vaccination Does Not Increase OxLDL or PC-Specific Antibody Titers}

To determine whether Prevenar 13 vaccination induces an immune response against oxLDL, IgM and IgG antibody titers against oxLDL (copper oxidized moiety) were measured. No differences were observed in anti-oxLDL IgM and IgG antibody levels in either the overall patient population or the patient subgroups four weeks after Prevenar 13 vaccination (Figure 3A-H; Table S1), suggesting that the Prevenar 13 vaccine does not elicit an immune response against oxLDL in these groups. Finally, due to molecular mimicry between PC-moiety of S. pneumoniae and oxLDL, we analyzed the effect of Prevenar 13 vaccination on PC-specific IgM and IgG levels. Surprisingly, four weeks after receiving a single dose of Prevenar 13, we observed a reduction in anti-PC IgM antibody levels in all patients (Figure 4A). In contrast, no differences were observed in PC-specific IgG antibody levels in patients following Prevenar vaccination (Figure 4B). In LPD patients, Prevenar 13 vaccination tended to reduce anti-PC IgM antibody titers, although this effect did not reach statistical significance; no differences were observed in LPD patients following Prevenar 13 vaccination regarding antiPC IgG antibody levels (Figure 4C,D). Regarding FH and NPB patients, Prevenar 13 did not elicit measurable changes in anti-PC IgM and IgG antibody levels (Figure 4E-H). More details on the statistical analysis are presented in Table S1. 
A

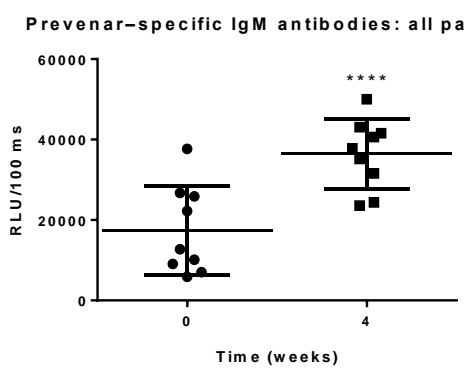

C

Prevenar-specific IgM antibodies: LPD patients

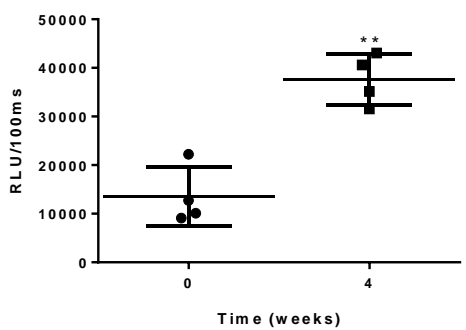

E

Prevenar-specific IgM antibodies: FH patients

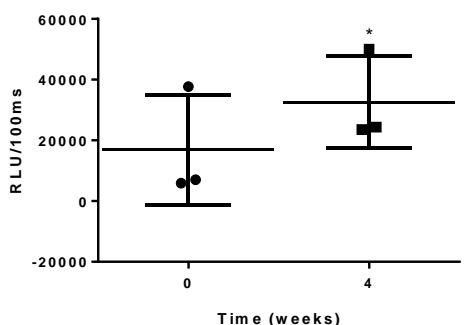

G

Prevenar-specific IgM antibodies: NPB patients

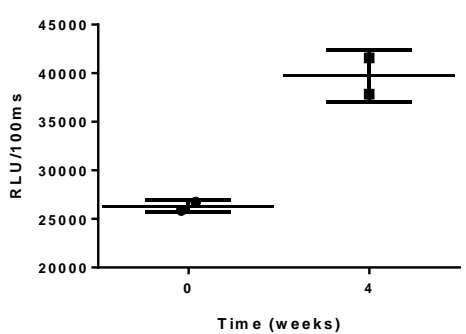

B

Prevenar-specific IgG antibodies: all patients

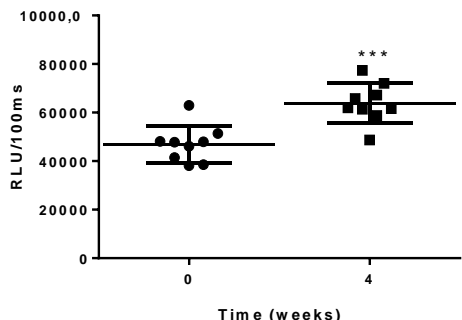

D

Prevenar-specific IgG antibodies: LPD patients

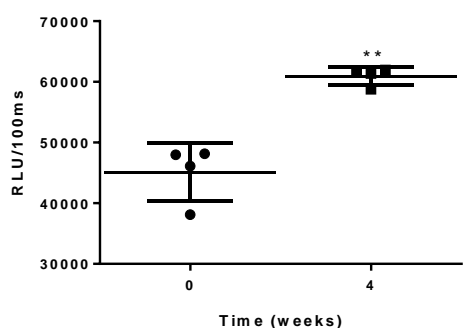

$\mathbf{F}$

Prevenar-specific IgG antibodies: FH patients

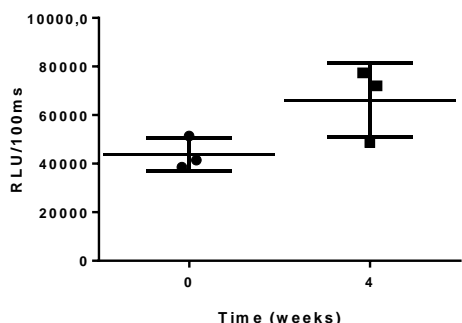

H

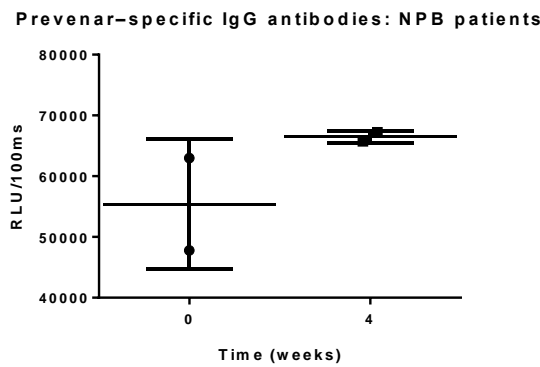

Figure 2. Prevenar-specific antibody titers. (A,B) Anti-Prevenar IgM and IgG antibody levels in all patients. (C,D) AntiPrevenar IgM and IgG antibody levels in LPD patients. (E,F) Anti-Prevenar IgM and IgG antibody levels in FH patients. $(\mathbf{G}, \mathbf{H})$ Anti-Prevenar IgM and IgG antibody levels in NPB patients. * Indicates $p<0.05$. All error bars represent SD. 
A

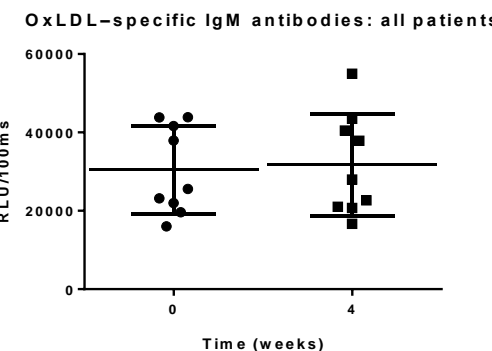

C

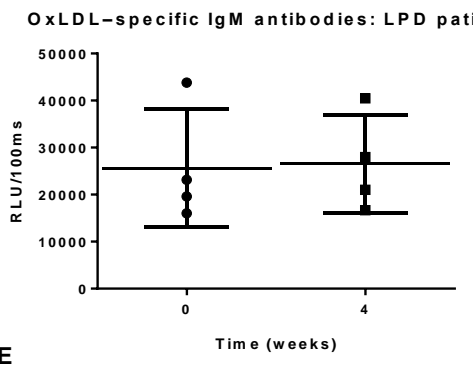

E

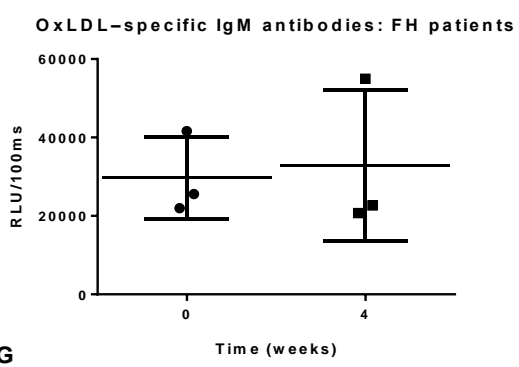

OxLDL-specific IgM antibodies: NPB patients

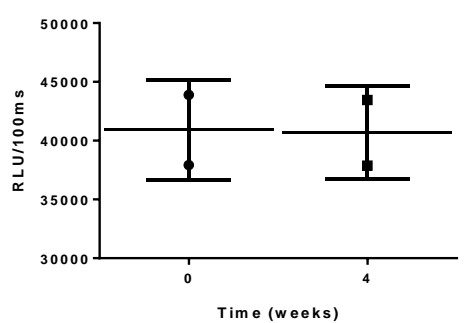

B

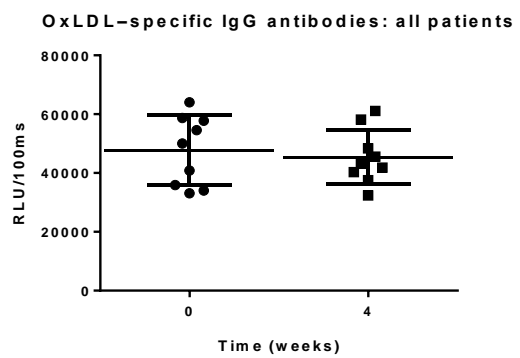

D
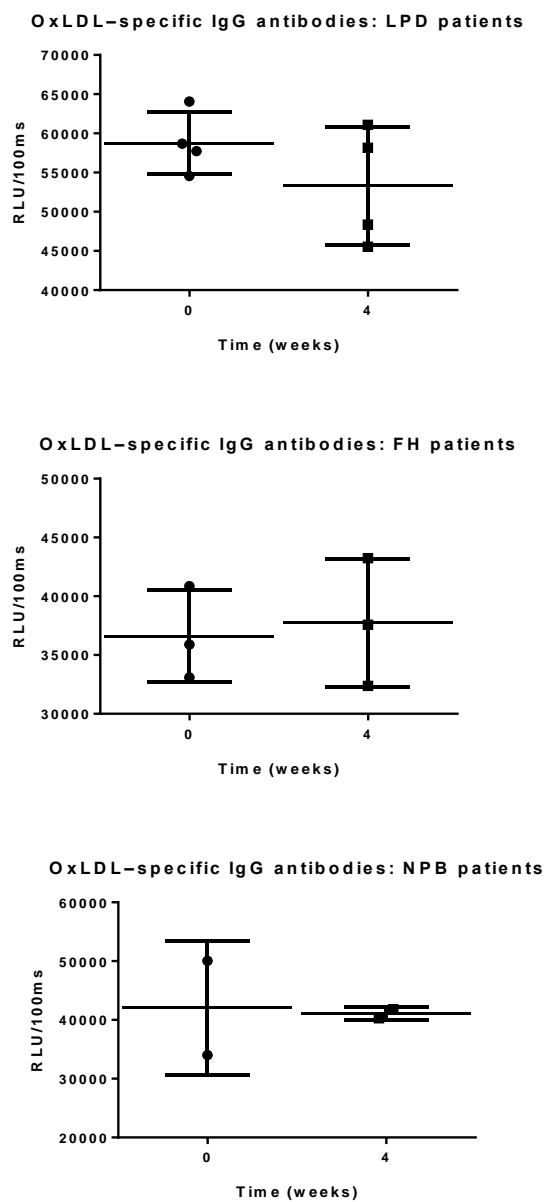

Figure 3. Oxidized low-density lipoprotein (OxLDL)-specific antibody titers (A-H). All error bars represent SD. 
A

PC-specific IgM antibodies: all patients

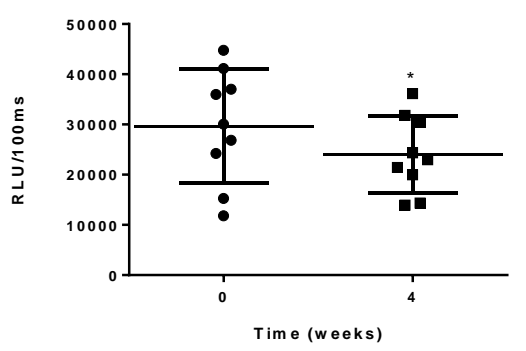

C

PC-specific IgM antibodies: LPD patients

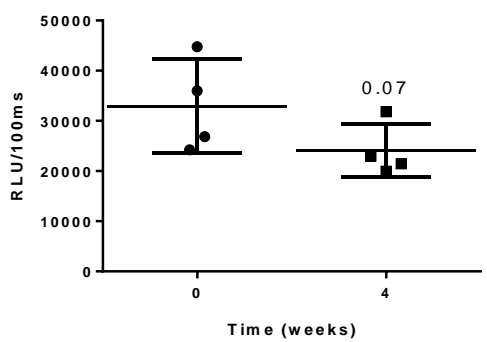

E

PC-specific IgM antibodies: FH patients

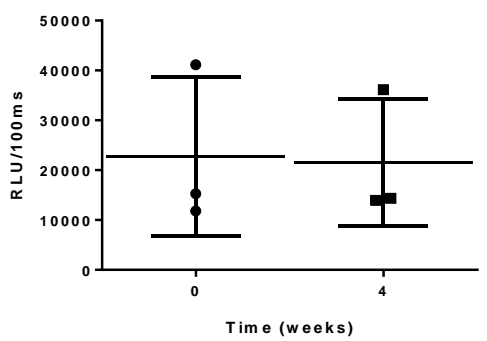

G

PC-specific IgM antibodies: NPB patients

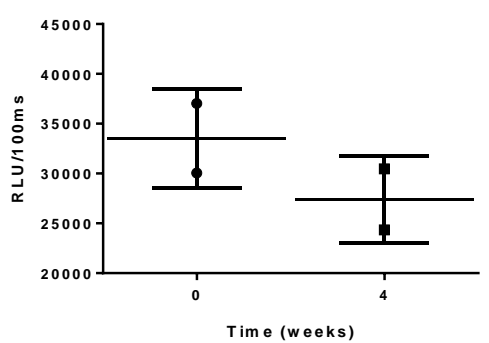

B

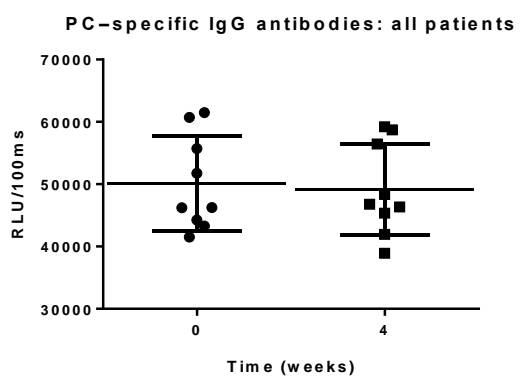

D

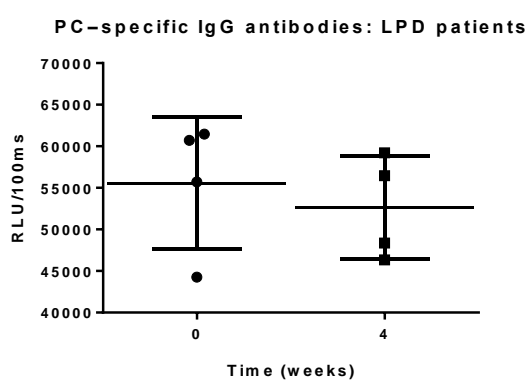

$\mathbf{F}$

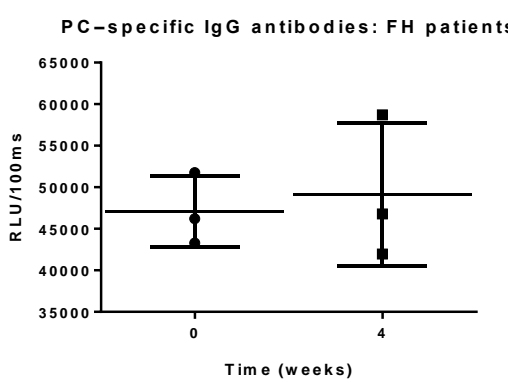

H

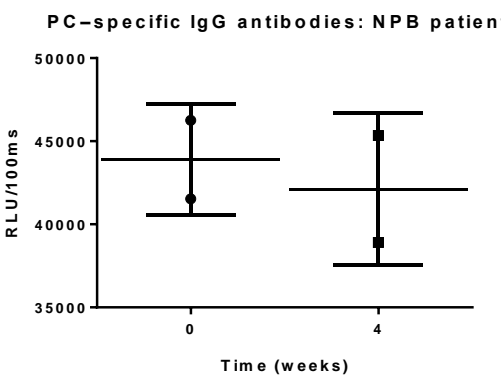

Figure 4. Phosphorylcholine (PC)-specific antibody titers (A-H). ${ }^{*}$ Indicates $p<0.05$. All error bars represent SD. 


\section{DISCUSSION}

In the current study, we analyzed whether a pneumococcal conjugate vaccine also elicits an immune response against oxLDL and/or PC in human metabolic disease patients. While patients displayed an appropriate IgM- and IgG-mediated immune response against the Prevenar 13 vaccine, Prevenar 13 vaccination did not increase antibody levels against oxLDL or PC in patients. These findings suggest that the Prevenar 13 vaccine is not a suitable tool to increase anti-oxLDL or PC antibodies in metabolic disease patients, and that alternative immunization strategies are needed in order to target oxLDL in humans. Further studies in larger, more homogenous populations should be performed to confirm our findings.

Oxidative stress, a phenomenon in which high amounts of reactive oxygen species overwhelm the cells' antioxidant defenses, is a hallmark of metabolic syndrome, as well as a wide range of metabolic and/or inflammatory diseases. As a lipid-rich aggregate, LDL is prone to oxidative modifications, and thus is susceptible to oxLDL conversion under oxidative stress conditions. OxLDL, in turn, interferes with cell metabolism and activates proinflammatory and apoptotic pathways in cells, further impairing cellular metabolism and function, exacerbating the production of reactive oxygen species and triggering a vicious pathogenic cycle. Depending on the oxidative reagents and mechanisms, different portions of LDL can be modified to different extents; as such, it is likely that a heterogeneous population of oxLDL coexists in living organisms at any given time. Accordingly, antibodies targeting different oxLDL epitopes have been described in animals and humans alike, including antibodies against MDA or PC, other epitopes in copper-oxidized LDL, and against specific apoliprotein B peptides (16). While several immunogenic groups are present in oxLDL, it is essential to establish the optimal oxLDL-related antigen for efficient immunization strategies, in order to avoid cross-reactions with non-pathogenic, non-oxLDL components. Being a common headgroup on phospholipids, PC is an abundant component of cell membranes and native LDL, but only becomes exposed and available as a so-called neo-antigen in apoptotic cells and oxLDL. In addition to being a neo-antigen, PC is a foreign antigen present on a variety of infectious agents, including S. pneumoniae. As such, among the different oxLDL immunogenic moieties, PC is an interesting candidate to explore, as (1) antiPC autoantibodies have been found in human sera, (2) human anti-PC autoantibodies crossreact with oxLDL, and (3) PC is unlikely to induce an immune response against healthy cells or otherwise harmless cell components (17).

Previously, mice whose anti-oxLDL IgM antibody levels were increased by means of heatinactivated S. pneumoniae displayed reduced disease burden in the context of NASH, NPC1 disease and atherosclerosis, strengthening the hypothesis that increasing anti-oxLDL 
antibody levels may be beneficial in humans. However, in contrast to observations in animal studies, in the current study, the Prevenar 13 vaccine did not increase anti-PC or anti-oxLDL antibody levels in metabolic disease patients. While we did observe a significant IgM and IgG-mediated response against Prevenar 13, it is possible that we could not measure a PC or oxLDL-specific immune response to the vaccine due to the limited sample size of the study and heterogenous composition regarding metabolic disease, sex and age of participants. In addition, it is possible that patients had received a pneumococcal vaccination in the past, which could impact their immune response against PC or oxLDL in the current study. Furthermore, it should be noted that, in the aforementioned in vivo studies, mice received an initial immunization consisting of heat-inactivated S. pneumoniae followed by several booster immunizations throughout the study period. In contrast, in this study, participants received a single dose of Prevenar 13 and were followed up for four weeks. As such, it is possible that a similar vaccination schedule is required to elicit an appropriate immune response against oxLDL and PC in humans. In addition, as already mentioned, mouse studies make use of heat-inactivated S. pneumoniae immunizations, in which the PC moiety is still present. In contrast, current pneumococcal vaccine manufacturing emphasizes the removal of PCcontaining cell wall polysaccharides, as antibodies against these bacteria components are not considered protective in humans (18). Therefore, available pneumococcal vaccines, including Prevenar, may not contain enough PC in their formulation to elicit an anti-PC and/or antioxLDL response. To address this issue, a study by Even et al. analyzed the effects of Prevenar vaccination on anti-PC antibody levels in apoliprotein $\mathrm{E}^{-/-}$mice (19). Following two Prevenar injections, the researchers observed an increase in anti-PC antibody levels, as well as reduced atherosclerotic burden in mice. While these results suggest that the Prevenar vaccination has enough residual PC in its composition to elicit an immune response, it is possible that vestigial PC amounts may differ between vaccine batches and therefore act as unreliable antigens (18). Finally, it should be noted that key differences in both innate and adaptive immunity of mice and humans may lead to different immune responses following exposure to the same pathogen/epitope (20). In addition, laboratory mice are kept in homogenous, aseptic and even sterile environments, which may also contribute to differences between immune responses of mice and humans to Prevenar 13 vaccination (21).

Previously, two studies reported that vaccination with a 23-valent polysaccharide vaccine (Pneumovax-23) has no effect on anti-MDA, anti-PC or anti- oxLDL in pediatric or adult patients $(18,22)$. While these findings appear to be in line with ours, it is worth mentioning that, while Prevenar 13 consists of 13 pneumococcal serotypes bound to a carrier that elicits a T-cell dependent immune response, Pneumovax-23 is a pneumococcal polysaccharide vaccine containing 23 S. pneumoniae serotypes whose immune response is T-cell independent. As such, Prevenar 13 elicits a stronger immune response than Pneumovax, particularly in 
young children and adults whose B-cells are not fully functioning (23). Therefore, it is possible that Pneumovax-23 is not effective enough to elicit an appropriate anti-PC or anti-oxLDL response. Of note, a recent report by Grievink H.W. et al. found that two Prevenar 13 vaccinations, 28 weeks apart, increased anti-oxLDL and anti-PC IgG and IgM antibody levels in males, compared to participants who only received placebo injections (24). It is possible that population size and heterogeneity, as well as the use of a single dose, did not allow the observation of a similar anti-oxLDL antibody increase following Prevenar 13 vaccination in the current study. Of note, the observed increase in anti-oxLDL antibody levels after two doses of Prevenar 13 in the discussed report reinforces the conclusion that Prevenar 13 composition retains molecular mimicry between S. pneumoniae and oxLDL, in line with a previously mentioned mouse study (19). Whereas in mice, pneumococcal immunization leads to a strongly IgM-mediated immune response, in humans an IgG-mediated immune response against PC has been predominantly observed, further emphasizing the importance of speciesrelated differences in the immune system (25). As such, it is possible that a pneumococcal vaccine would elicit a strong anti-oxLDL IgG response, as was described in the aforementioned reports $(24,25)$. Of note, whereas anti-oxLDL IgM antibodies are widely considered protective, anti-oxLDL IgG antibodies have been reported as pro-atherogenic. As such, the previous report raises questions regarding the therapeutic potential of pneumococcal vaccination against metabolic and inflammatory diseases.

Overall, our study suggests that a single dose of Prevenar 13 does not elicit an increase in anti-oxLDL or anti-PC IgM antibodies in metabolic disease patients. While further studies are required to confirm our findings, due to the limited sample size and heterogeneity of patients included in this pilot study, these results emphasize the need to explore alternative strategies to increase oxLDL clearance. 


\section{REFERENCES}

1. Rochlani Y, Pothineni NV, Kovelamudi S, Mehta JL. Metabolic syndrome: pathophysiology, management, and modulation by natural compounds. Therapeutic Advances in Cardiovascular Disease. 2017;11(8):215-25.

2. WHO. Noncommunicable diseases: Key Facts 2018. Available from: https://www.who.int/news-room/fact-sheets/detail/noncommunicable-diseases. Accessed on: $30 / 03 / 2020$

3. Levitan I, Volkov S, Subbaiah PV. Oxidized LDL: diversity, patterns of recognition, and pathophysiology. Antioxid Redox Signal. 2010;13(1):39-75.

4. Matsuura E, Hughes GRV, Khamashta MA. Oxidation of LDL and its clinical implication. Autoimmunity Reviews. 2008;7(7):558-66.

5. Omar NN, El Hefnawy MH, El Soda MF, Heider NM, Hamed HI. Assessment of oxLDL, anti-oxLDL antibodies and lipoprotein-associated phospholipase A2 as cardiovascular risk markers in obese adolescents with and without T1DM. Bulletin of Faculty of Pharmacy, Cairo University. 2017;55(2):325-31.

6. Holvoet P, De Keyzer D, Jacobs DR, Jr. Oxidized LDL and the metabolic syndrome. Future Lipidol. 2008;3(6):637-49.

7. Su J, Georgiades A, Wu R, Thulin T, de Faire U, Frostegård J. Antibodies of IgM subclass to phosphorylcholine and oxidized LDL are protective factors for atherosclerosis in patients with hypertension. Atherosclerosis. 2006;188(1):160-6.

8. De Faire U, Frostegård J. Natural Antibodies against Phosphorylcholine in Cardiovascular Disease. Annals of the New York Academy of Sciences. 2009;1173(1):292-300. 9. de Faire U, Su J, Hua X, Frostegård A, Halldin M, Hellenius M-L, et al. Low levels of IgM antibodies to phosphorylcholine predict cardiovascular disease in 60-year old men: Effects on uptake of oxidized LDL in macrophages as a potential mechanism. Journal of Autoimmunity. 2010;34(2):73-9.

10. Vas J, Grönwall C, Silverman G. Fundamental roles of the innate-like repertoire of natural antibodies in immune homeostasis. Frontiers in Immunology. 2013;4:4.

11. Binder CJ, Hörkkö S, Dewan A, Chang M-K, Kieu EP, Goodyear CS, et al. Pneumococcal vaccination decreases atherosclerotic lesion formation: molecular mimicry between Streptococcus pneumoniae and oxidized LDL. Nature Medicine. 2003;9(6):736-43.

12. Houben T, Magro dos Reis I, Oligschlaeger Y, Steinbusch H, Gijbels MJJ, Hendrikx $\mathrm{T}$, et al. Pneumococcal Immunization Reduces Neurological and Hepatic Symptoms in a Mouse Model for Niemann-Pick Type C1 Disease. Frontiers in Immunology. 2019;9:3089.

13. Bieghs V, van Gorp PJ, Walenbergh SMA, Gijbels MJ, Verheyen F, Buurman WA, et al. Specific immunization strategies against oxidized low-density lipoprotein: A novel way to reduce nonalcoholic steatohepatitis in mice. Hepatology. 2012;56(3):894-903. 
14. Sun X, Seidman JS, Zhao P, Troutman TD, Spann NJ, Que X, et al. Neutralization of Oxidized Phospholipids Ameliorates Non-alcoholic Steatohepatitis. Cell Metabolism. 2020;31(1):189-206.e8.

15. Hendrikx T, Watzenböck ML, Walenbergh SMA, Amir S, Gruber S, Kozma MO, et al. Low levels of IgM antibodies recognizing oxidation-specific epitopes are associated with human non-alcoholic fatty liver disease. BMC Med. 2016;14(1):107-.

16. Ylä-Herttuala S, Palinski W, Butler SW, Picard S, Steinberg D, Witztum JL. Rabbit and human atherosclerotic lesions contain IgG that recognizes epitopes of oxidized LDL. Arteriosclerosis and thrombosis : a journal of vascular biology. 1994;14(1):32-40.

17. Schenkein HA, Berry CR, Purkall D, Burmeister JA, Brooks CN, Tew JG. Phosphorylcholine-dependent cross-reactivity between dental plaque bacteria and oxidized low-density lipoproteins. Infect Immun. 2001;69(11):6612-7.

18. John T. Nguyen NM, Jana Palaia, Angeliki Georgopoulos, Jeffrey B. Rubins, Edward N. Janoff. Humoral responses to oxidized low-density lipoprotein and related bacterial antigens after pneumococcal vaccine. Translational Research. 2007;150(3):172-9.

19. Even G, Kiss M, Laschet J, Ozvar Kozma M, Simon T, Wigren M, et al. Vaccination with Prevenar ${ }^{\circledR}$ boosts the production of anti-phosphorylcholine antibodies and protects APOE knockout mice from atherosclerosis. Atherosclerosis. 2018;275:e6-e7.

20. Mestas J, Hughes CCW. Of Mice and Not Men: Differences between Mouse and Human Immunology. The Journal of Immunology. 2004;172(5):2731.

21. Tao L, Reese TA. Making Mouse Models That Reflect Human Immune Responses. Trends in Immunology. 2017;38(3):181-93.

22. Damoiseaux J, Rijkers G, Tervaert JWC. Pneumococcal vaccination does not increase circulating levels of IgM antibodies to oxidized LDL in humans and therefore precludes an anti-atherogenic effect. Atherosclerosis. 2007;190(1):10-1.

23. LaFon DC, Nahm MH. Measuring immune responses to pneumococcal vaccines. J Immunol Methods. 2018;461:37-43.

24. Grievink HW, Gal P, Ozsvar Kozma M, Klaassen ES, Kuiper J, Burggraaf J, et al. The Effect of a 13-Valent Conjugate Pneumococcal Vaccine on Circulating Antibodies Against Oxidized LDL and Phosphorylcholine in Man, A Randomized Placebo-Controlled Clinical Trial. Biology. 2020;9(11).

25. Carl E. Frasch NFC. Specificity of Human Antibodies Reactive with Pneumococcal C Polysaccharide. Infect Immun. 2000. 
Chapter 7

General discussion 
Lysosomal cholesterol accumulation underlies oxidative stress, metabolic dysfunction, inflammation and overall disease burden in NPC1 disease. In addition, lysosomal cholesterol accumulation is a feature of a wide number of acquired metabolic inflammation diseases, including NASH, atherosclerosis, Alzheimer's disease and several types of cancer. Therefore, lysosomal dysfunction, mediated by cholesterol accumulation, is an appealing and widely applicable target for the development of preventive and therapeutical strategies. Here, we aimed to investigate the role of NPC1 impairment on the gut microbiome, as well as the potential of different therapeutical strategies to reduce disease burden following lysosomal cholesterol accumulation. In this General Discussion, I'll proceed to discuss the findings presented on this thesis in light of other studies, as well as their impact on current knowledge and clinical applications.

\section{NPC1 impairment beyond NPC1 disease: role of NPC1 in metabolic inflammation, liver cholesterol biosynthesis and gut microbiome}

Similarly to the busy traffic in urban areas, cells are constantly producing and sending out signals and nutrients to each other, allowing cooperation between distal tissues and organs to regulate and support our bodies' activity. In addition, individual cells must carefully orchestrate a multitude of intracellular processes (f.i., DNA transcription and protein synthesis, building of structural/signalling molecules, breakdown and use of nutrients, etc) to ensure homeostasis. The proper function and viability of a cell thus depends on timely and accurate coordination between organelles and metabolic pathways, as well as on adequate responses to the extracellular environment. In this context, lysosomes are important integrators of multiple extra- and intracellular pathways and are essential for proper cell metabolism and function. As such, lysosomal dysfunction can have dire and far-reaching consequences for the organism, as is well illustrated in lysosomal storage diseases, such as NPC1 disease. NPC1 disease, a recessive autosomal disease, is caused by deleterious mutations in either NPC1 or NPC2 genes (in 95\% and 5\% of cases, respectively). Since both NPC1 and NPC2 are required for proper lysosomal cholesterol efflux, mutations in either NPC1 or NPC2 cause the same phenotype, i.e., lysosomal cholesterol accumulation. Overall, chronic lysosomal lipid accumulation following NPC1 dysfunction leads to disturbed cell cholesterol distribution, oxidative stress and inflammation via a variety of pathways, resulting in severe organ dysfunction and damage. While deleterious, autosomal-recessive mutations in NPC1, such as those causing NPC1 disease, are very rare, a growing body of findings has pinpointed several deleterious NPC1 heterozygous variants and SNPs in different human populations (1-4). Importantly, such variants are associated with weight gain, dyslipidaemia and insulin resistance. While the relationship between NPC1 $\mathrm{SNPs} /$ haploinsufficiency and metabolic dysfunction remains associative in humans, investigations in mouse models support a causal role for mild NPC1 dysfunction in metabolic 
disturbances. Indeed, studies analysing the impact of a high-caloric diet in $\mathrm{Npc1}^{+-}$mice found increased body mass, hepatic steatosis and adipose tissue hypertrophy, as well as insulin resistance, underlined by increased SREBP activation $(5,6)$. Taken together, the abovementioned findings highlight the role of (mild) NPC1 dysfunction and obesity and MetS traits.

In chapter 3 of this thesis, we observed higher levels of hepatic desmosterol, a sterol intermediate in the Bloch cholesterol synthesis pathway, in $N p c 1^{\text {nih }}$-tp mice compared to $N p c 1^{w t}$-tp mice. These findings suggest that NPC1 dysfunction in a model for metabolic inflammation shifts liver cholesterol metabolism, further emphasizing the role of NPC1 and lysosomal cholesterol in metabolic regulation. While it is unclear why cells have two distinct cholesterol synthesis pathways at their disposal, namely the Bloch and the Kandutsch-Russell pathways, studies indicate that intermediate sterols in each pathway exert different physiological responses in a cell-specific manner. For instance, increased accumulation of desmosterol levels has been shown to increase cholesterol efflux and to reduce cholesterol synthesis and inflammation in macrophages, but not hepatocytes. In addition, studies have shown increased desmosterol levels in NASH and atherosclerosis patients, further emphasizing the physiological relevance of distinct metabolic cholesterol synthesis in health and disease. In line with these observations, in chapter 3, following a HFC diet, we found increased liver desmosterol and reduced lathosterol levels (data not shown) in Npc1 ${ }^{\text {nih }}$-tp mice compared to $N p c 1^{w t}$-tp mice. While, to the best of our knowledge, the impact of Npc1 mutations on liver sterol composition has yet to be analysed, the aforementioned finding suggests that NPC1 impairment on blood-derived immune cells shifts cholesterol biosynthesis from the Kandutsch-Russell to the Bloch pathway in the liver, regardless of diet. Considering the previously mentioned findings regarding the role of desmosterol in metabolic dysfunction and inflammation, the observed increase of desmosterol in the livers of Npc1 $1^{\text {nih-tp }}$ mice following a HFC diet may reflect a compensatory mechanism by macrophages to attenuate a pro-inflammatory response to increased lysosomal lipid accumulation. Of note, while in a previous study plant stanol supplementation reduced liver desmosterol levels in $\mathrm{Ldll}^{- \text {- }}$ mice, in the current study, we observed a further increase in liver desmosterol levels of $N p c 1^{\text {nih }}$-tp mice following plant stanol supplementation to HFC diet. While the physiological relevance of such an increase is unclear, considering the accompanying reduction in liver inflammation, it is possible that plant stanol supplementation stimulated $N p c 1^{\text {nih }}$ macrophages to further accumulate desmosterol, thereby increasing LXR activation, inhibiting SREBP-pathways and reducing inflammatory responses. Overall, these findings highlight the role of hematopoietic NPC1 dysfunction in liver cholesterol biosynthesis and suggest that desmosterol accumulation may be beneficial 
in the context of metabolic inflammation following impaired NPC1 function and lysosomal cholesterol accumulation.

In addition to pinpointing hematopoietic NPC1 dysfunction as a regulator of cholesterol biosynthesis in the liver, in chapter 4 of this thesis, we further explored the impact of hematopoietic NPC1 dysfunction on gut microbiome composition in a model for metabolic inflammation disorders such as obesity and NASH. Our findings indicate that, in parallel with disturbed lipid metabolism and hepatic steatosis, $N p c 1^{\text {nih }}$-tp mice fed a HFC diet featured a more diverse gut microbiome than mice without a NPC1 defect. Of note, while genetic background has been shown to influence gut microbiome in mice $(7,8)$, in line with this study, such relationships are harder to establish in large human populations $(9,10)$, due to the wide range of factors influencing the gut microbiome (fi, age, sex, diet, exposition to environmental factors) that are more heterogeneous in human subjects compared to laboratory mice. Indeed, one study assessing the relationship between host genetics and gut microbiome in a large human cohort (11) found that, compared to extrinsic factors, genetic background is only minorly associated with the composition of gut microbiome. In other words, genetic screening in combination with pertinent environmental factors is likely to give more insight into the role of the gut microbiome in health and disease, rather the assessment of genetic factors alone. In line, our study in chapter 4 demonstrates that a hematopoietic Npc1 mutation interacts with a HFC diet in the context of metabolic inflammation to modulate gut microbiome composition, providing additional support for the interaction between host genetics, gut microbiome and diet. It is well known that the immune system modulates, and is modulated by, the gut microbiome $(12,13)$. Furthermore, defects in NPC1 in immune cells compromise their phagocytic ability and modulate their profile (14-17); as such, although findings in chapter 4 are of an associative nature only, it is likely that hematopoietic NPC1 dysfunction effectively influences the interaction between immune cells and the gut. Indeed, NPC1 dysfunction has been associated with impaired bacterial clearance by immune cells, due to deficient autophagy pathways (18). Of note, such a mechanism also underlies pathophysiology of inflammatory bowel syndrome, a disorder affecting a portion of NPC1 disease patient $(19,20)$. These findings indicate that host NPC1 dysfunction may impact the gut microbiome composition via impaired bacterial clearance of gut infiltrated immune cells, which in turn may play a role in gut pathology, similarly to inflammatory bowel syndrome $(21,22)$. In chapter 4 , we found that hematopoietic NPC1 dysfunction in Ldl $r^{--}$mice fed a HFC diet is associated with increased gut microbiome diversity; whether such an effect is underlined by poor clearance of specific bacterial species due to the aforementioned mechanism, remains unclear. Overall, future studies should validate findings from chapter 4 of this thesis in humans. Furthermore, it remains unclear whether our findings are applicable only to individuals with NPC1 mutations/variations, or if they can also be observed in patients 
with acquired lysosomal cholesterol accumulation, as observed in obesity and NASH. Finally, given the dual relationship between gut microbiota and host immune system, it would be interesting to analyse whether modulation of gut microbiome (fi, via dietary interventions, pre-/probiotic administration) yields therapeutic benefits for patients with metabolic inflammation underlined by lysosomal cholesterol accumulation (23).

\section{Beyond NPC1 dysfunction: oxLDL as a contributor to disease burden in NPC1 disease}

oxLDL, a product of oxidative modifications of native LDL, is observed in high amounts in a wide range of diseases, including NPC1 disease, NASH and, most notably, cardiovascular disease. While the process of oxLDL formation in vivo has yet to be fully established, studies suggest this can occur within dysregulated lysosomes via iron-metabolism components, as well as in the subendothelial space in the context of metabolic dysfunction and oxidative stress. Furthermore, rather than a single, well-defined molecule, the term "oxLDL" encompasses a wide range of LDL molecules that underwent oxidative modifications to different extents, on different molecular components (ie, TGs, phospholipids, apolipoproteins, free cholesterol and cholesterol esters). Among the different LDL components, polyunsaturated FAs in phospholipids are especially susceptible to oxidative modifications, and oxidized phosphatidylcholine (ie, phosphorylcholine, "PC") is commonly observed in oxLDL $(24,25)$. Aside from oxidative modifications, oxLDL differs from native or minimally modified LDL due to the fact that it is unable to bind to LDL receptors; instead, it is recognized by scavenger receptors, Toll-like receptors and lectin-type oxidized LDL receptors, triggering pro-inflammatory and apoptotic cascades within cells. In addition, uptake of oxLDL leads to lysosomal lipid accumulation, as lysosomes are not able to efficiently breakdown and efflux oxidized molecules. Overall, oxLDL lysosomal entrapment culminates in the formation of cholesterol crystals, dysregulation of lysosomal $\mathrm{pH}$ and lysosomal function, and cell apoptosis and inflammation. As such, oxLDL is a mediator of a vicious cycle, in which cell dysfunction and oxidative stress/inflammatory responses trigger oxLDL production, which in turn leads to further damage and oxLDL formation. Since lipid metabolism/lysosomal dysfunction, oxidative stress and inflammation are a hallmark of several diseases, as aforementioned, oxLDL may prove to be a promising, widely applicable diagnosis/therapeutical target.

Indeed, research indirectly analysing the role of oxLDL in human disease found that high levels of anti-oxLDL autoantibodies (ie, antibodies produced from birth in individuals) are protective against cardiovascular disease. Conversely, low anti-oxLDL autoantibodies are associated with worse prognosis in the same context. Further studies in mouse models for $\mathrm{NASH}$ and atherosclerosis found that increasing anti-oxLDL antibody levels via pneumococcal immunization ameliorates lipid metabolism and inflammation, strengthening 
the pathogenic role of oxLDL in the context of metabolic inflammation and highlighting the benefits of anti-oxLDL antibodies. In chapter 5 of this thesis, we further investigated whether increasing anti-oxLDL IgM antibodies via pneumococcal immunization can ameliorate systemic and neurological features of NPC1 disease. Similarly to the abovementioned studies in NASH and atherosclerosis, pneumococcal immunization reduced hepatic cholesterol accumulation and improved hepatic lipid metabolism and inflammation. In addition, pneumococcal immunization reduced the levels of apoptotic markers in the liver, suggesting either (1) reduced liver apoptosis and/or (2) improved clearance of apoptotic cells. Considering that pneumococcal immunization triggers the production of antibodies targeting oxidized PC, a component exposed on both oxLDL and apoptotic cells, it is unsurprising that pneumococcal immunization reduced hepatic apoptosis markers. As such, observed improvements in liver inflammation may also be attributed to reduced number of apoptotic cells, in addition to the increased clearance of oxLDL by anti-oxLDL IgM antibodies. Of note, it remains unclear how the increase in anti-oxLDL antibody levels triggers its clearance and protects immune cells from oxLDL-related toxicity. While one study proposed that antioxLDL antibodies increase the uptake of oxLDL by monocytes, several others came to the opposite conclusion, i.e., that anti-oxLDL IgM antibodies inhibit macrophage oxLDL uptake via scavenger receptors, thus preventing oxLDL-mediated dysfunction and reducing inflammation $(26,27)$. In addition, it should be emphasized that, while anti-oxLDL IgG antibodies activate pro-inflammatory cascades in cells and may exacerbate disease burden, IgM antibodies trigger anti-inflammatory pathways and are thus more protective than the former. Regardless of the underlying mechanisms, our findings from chapter $\mathbf{5}$ indicate that triggering the production of anti-oxLDL IgM antibodies via pneumococcal immunization improves systemic lipid accumulation and inflammation following NPC1 impairment.

While the impact of oxLDL in brain disorders has not been fully elucidated, previous studies have found increased levels of oxLDL in patients with Alzheimer's disease and cerebrovascular infarcts $(28,29)$, suggesting that oxLDL is associated with neurological disease progression. In line, we observed reduced neuroinflammation and improvements in motor skills in immunized mice with higher anti-oxLDL IgM antibody titres, further strengthening the relationship between circulating oxLDL and neurological damage. Since antibodies are usually precluded from the nervous system due to the BBB, it is unclear how pneumococcal immunization benefits NPC1 disease neurological symptoms. Previous studies indicate that high levels of oxLDL compromise the BBB integrity and are toxic to glial cells and neurons $(30,31)$. As such, it is possible that pneumococcal immunization benefits neurological symptoms of NPC1 disease by indirectly preventing and/or reducing oxLDLassociated damage to the BBB and associated exacerbation of disease burden. In addition, it 
is possible that a small amount of anti-oxLDL IgM antibodies can cross the BBB, particularly in case of BBB damage, thus directly modulating brain cells and reducing neuroinflammation.

\section{Targeting inflammation and oxidative stress in NPC1 and metabolic disease}

Although inflammation, oxidative stress and oxLDL formation are not the core pathological triggers of NPC1 disease, a large body of research, including chapter $\mathbf{5}$ of this thesis, indicates that such mechanisms are important contributors of disease burden. While directly correcting NPC1 deficiency is the most straightforward therapeutic strategy in NPC1 disease, particularly in patients with missense NPC1 mutations, strategies such as gene therapy, while beneficial in in vivo models $(32,33)$, have yet to be perfected efficacy and safety-wise and analysed in human patients. Currently, Miglustat, a glucosylceramide synthase inhibitor, is the only therapeutical compound approved for treatment of NPC1 disease. While the use of Miglustat can reduce disease progression and improve life quality of NPC1 disease patients, its benefits are still limited, particularly in patients diagnosed later in life or with more aggressive disease phenotype (34-36). Besides Miglustat, intrathecal administration of cyclodextrin is another promising compound to reduce NPC1 disease progression and ameliorate associated symptoms, although further clinical investigations are required for its approval $(37,38)$. Overall, considering the limitations in current therapeutic strategies for NPC1 disease, the development of alternative strategies, while not curative, may further contribute to reduce disease progression and burden.

In line, in chapters $\mathbf{2}$ and $\mathbf{3}$, we demonstrated that the use of plant stanols improves systemic lipid metabolism and inflammation following whole body or hematopoietic NPC1 defects. Of note, we did not directly analyse the effect of plant stanol supplementation on lysosomal cholesterol content in either chapter 2 or chapter 3 . Such analyses would further help to pinpoint whether benefits of increased plant stanol consumption stem directly from reduced lysosomal cholesterol accumulation, or rather from improving secondary pathological features following NPC1 dysfunction. Furthermore, while our observations indicate that dietary plant stanol supplementation improves hepatic lipid metabolism and reduces inflammatory phenotype of liver and circulating immune populations, we did not analyse the effects of plant stanol supplementation on neurological pathological features of NPC1 disease in chapter 2. Previously, two independent investigations showed that plant stanols and sterols accumulate in the brain following dietary supplementation with these compounds in mice and rabbits $(39,40)$. Of note, plant stanols were found to accumulate at much lower rates than plant sterols after 6 weeks of dietary supplementation, likely due to higher side chain complexity of plant stanol molecules compared to that of plant sterols. As such, it is unlikely that dietary plant stanol supplementation elicits a functionally-relevant accumulation of these molecules in the nervous system. Of note, while stigmasterol may reduce neurodegeneration 
in Alzheimer's disease, administration of other plant sterols commonly consumed in a healthy diet were found to increase $A \beta$ production and secretion in an in vitro model (41). Furthermore, plant sterols are more prone to oxidative modifications than plant stanols (42, 43). Considering the disease burden associated with oxidative stress, these findings suggest that plant sterol supplementation may be ill-advised in NPC1 disease. Overall, further studies are required to confirm the rate of diet-derived plant stanols' accumulation in the brain, as well as the effects of plant stanols in brain lipid metabolism, inflammation and function. Furthermore, it is unclear whether pharmacological interventions with similar effects as plant stanols, such as ezetimibe, may yield better results in ameliorating lysosomal cholesterol accumulation-related metabolic dysfunction and inflammation.

In addition to highlighting the benefits of plant stanol supplementation following NPC1 dysfunction, findings from chapter 5 emphasize the pathological role of oxLDL in systemic and neurological damage in a mouse model for NPC1 disease. As such, targeting oxLDL may prove valuable to prevent or reduce disease burden associated underlined by lysosomal lipid accumulation, oxidative stress and inflammation. Whether such a strategy can be successfully employed in human patients remains to be elucidated. In chapter 6, we demonstrated that pneumococcal vaccination does not increase anti-oxLDL antibody levels in patients with metabolic diseases underlined by dyslipidaemia, oxidative stress and inflammation. While our sample size was small and composed of a heterogeneous population, our observations are in line with several other studies. As such, it is unlikely that administering commonly used pneumococcal vaccines can be used as tool to increasing anti-oxLDL IgM antibody levels and subsequently reduce circulating oxLDL in human patients. To date, no other strategies directly targeting oxLDL have been explored in humans, although the use of antioxidants to reduce disease burden in different contexts has been extensively researched. Of note, while in vivo studies have found antioxidant tools to benefit cardiovascular, NPC1 and Alzheimer's disease symptoms, such strategies in human patients have largely fallen short of expectations (44-47). Rather than indicating that targeting secondary disease mechanisms should be dismissed as a therapeutic strategy, the limited success of tools employed so far to that end emphasizes the need to explore more efficient methods to reduce oxidative stress and inflammation. Indeed, while the reasons underlying the discrepancy between the success of antioxidants in in vivo studies and clinical trials are not fully known, they likely include: prooxidant effects of traditional antioxidants such as vitamin E; inability of traditional antioxidants to penetrate cells and mitochondria, particularly when the latter are compromised; as well as premature antioxidant breakdown and interaction with other medications. The aforementioned mechanisms and caveats should thus be considered while developing therapeutic strategies targeting oxidative stress in humans. Finally, it should be emphasized that, while oxidative stress and inflammation are secondary to lysosomal 
cholesterol buildup in NPC1 disease, such mechanisms also trigger lysosomal lipid accumulation, for instance by increasing oxLDL production, among other pathological mechanisms. As such, anti-inflammatory and antioxidant strategies may be particularly beneficial in metabolic diseases underlined by metabolic dysfunction, such as in lifestyle and NPC1-variants driven obesity and MetS and other non-communicable diseases, including cardiovascular and liver disease, cancer and Alzheimer's disease. 
Table 1 Main findings, limitations and applications of each experimental chapter in this thesis.

\begin{tabular}{|c|c|c|c|}
\hline Chapter & Main findings & Limitations & Applications \\
\hline Chapter 2 & $\begin{array}{l}\text { Supplementing the diet with } \\
\text { plant stanols, particularly at a } \\
\text { higher dose, improves } \\
\text { systemic features of NPC1 } \\
\text { disease, namely: progressive } \\
\text { weight loss; hepatic } \\
\text { cholesterol accumulation and } \\
\text { lipid metabolism; hepatic and } \\
\text { blood inflammation } \\
\text { parameters. }\end{array}$ & $\begin{array}{l}\text { Did not investigate } \\
\text { effects of plant stanol } \\
\text { supplementation on } \\
\text { the nervous system }\end{array}$ & $\begin{array}{l}\text { As a } \\
\text { complementary } \\
\text { therapeutic tool, } \\
\text { dietary plant } \\
\text { stanol } \\
\text { supplementation } \\
\text { may improve } \\
\text { systemic NPC1 } \\
\text { disease } \\
\text { symptoms. . }\end{array}$ \\
\hline Chapter 3 & $\begin{array}{l}\text { - Following a HFC diet in a } \\
\text { murine model for metabolic } \\
\text { inflammation, plant stanol } \\
\text { supplementation ameliorates } \\
\text { hepatic cholesterol } \\
\text { accumulation, lipid } \\
\text { metabolism and } \\
\text { inflammation. In contrast, no } \\
\text { significant effects were } \\
\text { observed in the } \\
\text { abovementioned parameters } \\
\text { following plant stanol } \\
\text { supplementation to a } \\
\text { cholesterol poor, chow diet. } \\
\text { - These opposing findings } \\
\text { suggest that beneficial effects } \\
\text { of dietary plant stanol } \\
\text { supplementation are largely } \\
\text { dependent on excessive } \\
\text { cholesterol consumption. }\end{array}$ & $\begin{array}{l}\text { Could not fully } \\
\text { exclude direct anti- } \\
\text { inflammatory effects } \\
\text { of plant stanols, } \\
\text { mainly because: } \\
\text { - Did not investigate } \\
\text { the use of a higher } \\
\text { plant stanol } \\
\text { concentration (fi, } 6 \% \text { ). } \\
\text { - Did not investigate } \\
\text { the prolonged use of } \\
\text { plant stanol } \\
\text { supplementation. } \\
\text { - Hepatic lipid } \\
\text { metabolism } \\
\text { impairment and } \\
\text { inflammatory status } \\
\text { was much more } \\
\text { subtle in the analyzed } \\
\text { model following a } \\
\text { cholesterol poor diet } \\
\text { compared to a high } \\
\text { cholesterol diet. }\end{array}$ & $\begin{array}{l}\text { It is likely that } \\
\text { individuals who } \\
\text { consume } \\
\text { excessive } \\
\text { cholesterol will } \\
\text { benefit the most } \\
\text { from dietary } \\
\text { plant stanol } \\
\text { supplementation. }\end{array}$ \\
\hline
\end{tabular}




\begin{tabular}{|c|c|c|c|}
\hline Chapter 4 & $\begin{array}{l}\text { Hematopoietic Npc1 } \\
\text { mutation shifts gut } \\
\text { microbiome composition in a } \\
\text { mouse model for metabolic } \\
\text { inflammation, suggesting } \\
\text { that host genetics/lysosomal } \\
\text { lipid accumulation in } \\
\text { immune cells play a role in } \\
\text { gut microbiome. }\end{array}$ & $\begin{array}{l}\text { - Without human } \\
\text { data, translation of } \\
\text { these findings is } \\
\text { difficult. } \\
\text { - To strengthen the } \\
\text { findings, similar } \\
\text { experiments should } \\
\text { be conducted in } \\
\text { other mouse models } \\
\text { showing metabolic } \\
\text { inflammation. }\end{array}$ & $\begin{array}{l}\text { Therapies aimed } \\
\text { at improving } \\
\text { NPC1 } \\
\text { dysfunction } \\
\text { could improve } \\
\text { metabolic } \\
\text { inflammation by } \\
\text { modulating gut } \\
\text { microbiota. }\end{array}$ \\
\hline Chapter 5 & $\begin{array}{l}\text { Targetting oxLDL via } \\
\text { pneumococcal immunization } \\
\text { systemic and neurological } \\
\text { cholesterol accumulation, } \\
\text { inflammation as well as } \\
\text { neurological symptoms in a } \\
\text { mouse model for NPC1 } \\
\text { disease. }\end{array}$ & $\begin{array}{l}\text { - Addition of human } \\
\text { data would have } \\
\text { improved the } \\
\text { translational character } \\
\text { of the study. }\end{array}$ & $\begin{array}{l}\text { Increasing anti- } \\
\text { oxLDL IgM } \\
\text { antibodies may } \\
\text { be an effective } \\
\text { strategy to } \\
\text { improve } \\
\text { symptoms and } \\
\text { life quality of } \\
\text { NPC1 disease } \\
\text { patients. }\end{array}$ \\
\hline Chapter 6 & $\begin{array}{l}\text { A single dose of a conjugated } \\
\text { pneumococcal vaccine does } \\
\text { not increase IgM or IgG } \\
\text { antibody levels against } \\
\text { oxLDL in metabolic disease } \\
\text { patients. }\end{array}$ & $\begin{array}{l}\text { - Limited sample size. } \\
\text { - Heterogeneous } \\
\text { patient composition. }\end{array}$ & $\begin{array}{l}\text { Alternative } \\
\text { vaccination } \\
\text { strategies and/or } \\
\text { tools are } \\
\text { required to elicit } \\
\text { appropriate } \\
\text { responses } \\
\text { against oxLDL in } \\
\text { human metabolic } \\
\text { disease patients. }\end{array}$ \\
\hline
\end{tabular}




\section{REFERENCES}

1. Al-Daghri NM, Cagliani R, Forni D, Alokail MS, Pozzoli U, Alkharfy KM, et al. Mammalian NPC1 genes may undergo positive selection and human polymorphisms associate with type 2 diabetes. BMC Med. 2012;10(1):140.

2. Liu R, Zou Y, Hong J, Cao M, Cui B, Zhang H, et al. Rare Loss-of-Function Variants in \&lt;em\&gt;NPC1\&lt;/em\&gt; Predispose to Human Obesity. Diabetes. 2017;66(4):935.

3. Lamri A, Pigeyre M, Garver WS, Meyre D. The Extending Spectrum of NPC1Related Human Disorders: From Niemann-Pick C1 Disease to Obesity. Endocrine Reviews. 2018;39(2):192-220.

4. Chiorean A, Garver WS, Meyre D. Signatures of natural selection and ethnic-specific prevalence of NPC1 pathogenic mutations contributing to obesity and Niemann-Pick disease type C1. Scientific Reports. 2020;10(1):18787.

5. Castillo JJ, Jelinek D, Wei H, Gannon NP, Vaughan RA, Horwood LJ, et al. The Niemann-Pick C1 gene interacts with a high-fat diet to promote weight gain through differential regulation of central energy metabolism pathways. American Journal of Physiology-Endocrinology and Metabolism. 2017;313(2):E183-E94.

6. Jelinek D, Millward V, Birdi A, Trouard TP, Heidenreich RA, Garver WS. Npc1 haploinsufficiency promotes weight gain and metabolic features associated with insulin resistance. Human Molecular Genetics. 2011;20(2):312-21.

7. Snijders AM, Langley SA, Kim Y-M, Brislawn CJ, Noecker C, Zink EM, et al. Influence of early life exposure, host genetics and diet on the mouse gut microbiome and metabolome. Nature Microbiology. 2016;2(2):16221.

8. Org E, Parks BW, Joo JWJ, Emert B, Schwartzman W, Kang EY, et al. Genetic and environmental control of host-gut microbiota interactions. Genome Res. 2015;25(10):1558-69. 9. Turpin W, Espin-Garcia O, Xu W, Silverberg MS, Kevans D, Smith MI, et al. Association of host genome with intestinal microbial composition in a large healthy cohort. Nature Genetics. 2016;48(11):1413-7.

10. Davenport ER, Cusanovich DA, Michelini K, Barreiro LB, Ober C, Gilad Y. GenomeWide Association Studies of the Human Gut Microbiota. PLOS ONE. 2015;10(11):e0140301.

11. Rothschild D, Weissbrod O, Barkan E, Kurilshikov A, Korem T, Zeevi D, et al. Environment dominates over host genetics in shaping human gut microbiota. Nature. 2018;555(7695):210-5.

12. Durack J, Lynch SV. The gut microbiome: Relationships with disease and opportunities for therapy. J Exp Med. 2019;216(1):20-40.

13. Wang B, Yao M, Lv L, Ling Z, Li L. The Human Microbiota in Health and Disease. Engineering. 2017;3(1):71-82. 
14. Sergin I, Evans TD, Razani B. Degradation and beyond: the macrophage lysosome as a nexus for nutrient sensing and processing in atherosclerosis. Curr Opin Lipidol. 2015;26(5):394-404.

15. Viaud M, Ivanov S, Vujic N, Duta-Mare M, Aira L-E, Barouillet T, et al. Lysosomal Cholesterol Hydrolysis Couples Efferocytosis to Anti-Inflammatory Oxysterol Production. Circulation Research. 2018;122(10):1369-84.

16. Huang SC-C, Everts B, Ivanova Y, O'Sullivan D, Nascimento M, Smith AM, et al. Cell-intrinsic lysosomal lipolysis is essential for alternative activation of macrophages. Nature Immunology. 2014;15(9):846-55.

17. Deretic V. Autophagy in inflammation, infection, and immunometabolism. Immunity. 2021;54(3):437-53.

18. Schwerd T, Pandey S, Yang H-T, Bagola K, Jameson E, Jung J, et al. Impaired antibacterial autophagy links granulomatous intestinal inflammation in Niemann-Pick disease type C1 and XIAP deficiency with NOD2 variants in Crohn\&\#039;s disease. Gut. 2017;66(6):1060.

19. Kanegane H. Inflammatory bowel diseases and primary immunodeficiency diseases. Immunological Medicine. 2018;41(4):154-61.

20. Cavounidis A, Uhlig HH. Crohn's Disease in Niemann-Pick Disease Type C1: Caught in the Cross-Fire of Host-Microbial Interactions. Digestive Diseases and Sciences. 2018;63(4):811-3.

21. Menees S, Chey W. The gut microbiome and irritable bowel syndrome. F1000Res. 2018;7:F1000 Faculty Rev-29.

22. Chong PP, Chin VK, Looi CY, Wong WF, Madhavan P, Yong VC. The Microbiome and Irritable Bowel Syndrome - A Review on the Pathophysiology, Current Research and Future Therapy. Front Microbiol. 2019;10:1136-.

23. Salem AE, Singh R, Ayoub YK, Khairy AM, Mullin GE. The gut microbiome and irritable bowel syndrome: State of art review. Arab Journal of Gastroenterology. 2018;19(3):136-41.

24. Levitan I, Volkov S, Subbaiah PV. Oxidized LDL: diversity, patterns of recognition, and pathophysiology. Antioxid Redox Signal. 2010;13(1):39-75.

25. Hendrikx T, Binder CJ. Oxidation-Specific Epitopes in Non-Alcoholic Fatty Liver Disease. Front Endocrinol (Lausanne). 2020;11:941.

26. Binder CJ, Hörkkö S, Dewan A, Chang M-K, Kieu EP, Goodyear CS, et al. Pneumococcal vaccination decreases atherosclerotic lesion formation: molecular mimicry between Streptococcus pneumoniae and oxidized LDL. Nature Medicine. 2003;9(6):736-43.

27. Shaw PX, Hörkkö S, Tsimikas S, Chang M-K, Palinski W, Silverman GJ, et al. Human-Derived Anti-Oxidized LDL Autoantibody Blocks Uptake of Oxidized LDL by 
Macrophages and Localizes to Atherosclerotic Lesions In Vivo. Arterioscler Thromb Vasc Biol. 2001;21(8):1333-9.

28. Shie F-S, Neely MD, Maezawa I, Wu H, Olson SJ, Jürgens G, et al. Oxidized LowDensity Lipoprotein Is Present in Astrocytes Surrounding Cerebral Infarcts and Stimulates Astrocyte Interleukin-6 Secretion. The American Journal of Pathology. 2004;164(4):1173-81.

29. Montine TJ, Montine KS, Swift LL. Central nervous system lipoproteins in Alzheimer's disease. The American journal of pathology. 1997;151(6):1571-5.

30. Schreurs MPH, Cipolla MJ. Cerebrovascular dysfunction and blood-brain barrier permeability induced by oxidized LDL are prevented by apocynin and magnesium sulfate in female rats. Journal of cardiovascular pharmacology. 2014;63(1):33-9.

31. Lénárt N, Walter FR, Bocsik A, Sántha P, Tóth ME, Harazin A, et al. Cultured cells of the blood-brain barrier from apolipoprotein B-100 transgenic mice: effects of oxidized lowdensity lipoprotein treatment. Fluids and Barriers of the CNS. 2015;12(1):17.

32. Chandler RJ, Williams IM, Incao AA, Porter FD, Pavan WJ, Venditti CP. 198. AdenoAssociated Viral Gene Therapy To Treat Niemann-Pick Disease, Type C1. Molecular Therapy. 2015;23:S79.

33. Jiang D, Lee H, Pardridge WM. Plasmid DNA gene therapy of the Niemann-Pick C1 mouse with transferrin receptor-targeted Trojan horse liposomes. Scientific Reports. 2020;10(1):13334.

34. Fecarotta S, Romano A, Della Casa R, Del Giudice E, Bruschini D, Mansi G, et al. Long term follow-up to evaluate the efficacy of miglustat treatment in Italian patients with Niemann-Pick disease type C. Orphanet Journal of Rare Diseases. 2015;10(1):22.

35. Pineda M, Walterfang M, Patterson MC. Miglustat in Niemann-Pick disease type C patients: a review. Orphanet journal of rare diseases. 2018;13(1):140-.

36. Pineda M, Juríčková K, Karimzadeh P, Kolnikova M, Malinova V, Insua JL, et al. Disease characteristics, prognosis and miglustat treatment effects on disease progression in patients with Niemann-Pick disease Type C: an international, multicenter, retrospective chart review. Orphanet Journal of Rare Diseases. 2019;14(1):32.

37. Ory DS, Ottinger EA, Farhat NY, King KA, Jiang X, Weissfeld L, et al. Intrathecal 2hydroxypropyl-beta-cyclodextrin decreases neurological disease progression in NiemannPick disease, type C1: a non-randomised, open-label, phase 1-2 trial. Lancet. 2017;390(10104):1758-68.

38. Carradori D, Chen H, Werner B, Shah AS, Leonardi C, Usuelli M, et al. Investigating the Mechanism of Cyclodextrins in the Treatment of Niemann-Pick Disease Type $C$ Using Crosslinked 2-Hydroxypropyl- $\beta$-cyclodextrin. Small. 2020;16(46):2004735.

39. Fricke CB, Schrøder M, Poulsen M, von Bergmann K, Wester I, Knudsen I, et al. Increased plant sterol and stanol levels in brain of Watanabe rabbits fed rapeseed oil derived plant sterol or stanol esters. British Journal of Nutrition. 2007;98(5):890-9. 
40. Jansen PJ, Lütjohann D, Abildayeva K, Vanmierlo T, Plösch T, Plat J, et al. Dietary plant sterols accumulate in the brain. Biochimica et Biophysica Acta (BBA) - Molecular and Cell Biology of Lipids. 2006;1761(4):445-53.

41. Burg VK, Grimm HS, Rothhaar TL, Grösgen S, Hundsdörfer B, Haupenthal VJ, et al. Plant Sterols the Better Cholesterol in Alzheimer\&\#039;s Disease? A Mechanistical Study. The Journal of Neuroscience. 2013;33(41):16072.

42. Vanmierlo T, Husche C, Schött HF, Pettersson H, Lütjohann D. Plant sterol oxidation products - Analogs to cholesterol oxidation products from plant origin? Biochimie. 2013;95(3):464-72.

43. Plat J, Brzezinka H, Lütjohann D, Mensink RP, von Bergmann K. Oxidized plant sterols in human serum and lipid infusions as measured by combined gas-liquid chromatography-mass spectrometry. Journal of Lipid Research. 2001;42(12):2030-8.

44. Mitra S, Deshmukh A, Sachdeva R, Lu J, Mehta JL. Oxidized Low-Density Lipoprotein and Atherosclerosis Implications in Antioxidant Therapy. The American Journal of the Medical Sciences. 2011;342(2):135-42.

45. Singh SK, Srikrishna S, Castellani RJ, Perry G. Antioxidants in the Prevention and Treatment of Alzheimer's Disease. In: Al-Gubory KH, Laher I, editors. Nutritional Antioxidant Therapies: Treatments and Perspectives. Cham: Springer International Publishing; 2017. p. 523-53.

46. Teixeira JP, de Castro AA, Soares FV, da Cunha EFF, Ramalho TC. Future Therapeutic Perspectives into the Alzheimer's Disease Targeting the Oxidative Stress Hypothesis. Molecules. 2019;24(23):4410.

47. Fu R, Wassif CA, Yanjanin NM, Watkins-Chow DE, Baxter LL, Incao A, et al. Efficacy of N-acetylcysteine in phenotypic suppression of mouse models of Niemann-Pick disease, type C1. Human Molecular Genetics. 2013;22(17):3508-23. 



\section{Addendum}

Summary

Samenvatting

Impact paragraph

Acknowledgements

Curriculum vitae 


\section{Summary}

In recent years, lysosomal dysfunction has increasingly been acknowledged as a trigger and a contributing factor to a wide range of diseases, including NASH, atherosclerosis, dementia and several types of cancer. Among different mechanisms underlying lysosomal dysfunction, chronic lysosomal cholesterol buildup is of particular interest in the current age, as the number of individuals with obesity/overweight and MetS has been steadily increasing worldwide, in adults and children alike. Metabolic disturbances following chronic unhealthy lifestyles (i.e., excessive calory consumption in parallel with reduced physical activity) can trigger lysosomal cholesterol buildup, resulting in lysosomal and cellular dysfunction, inflammation and disease. In addition, deleterious mutations or SNPs compromising the activity of lysosomal cholesterol efflux protein NPC1 predispose individuals to lysosomal cholesterol accumulation, metabolic dysfunction and inflammation, as is well shown in the severity of NPC1 disease. As such, exploring mechanisms contributing to lysosomal cholesterol accumulation may shed light to develop further therapeutic strategies to prevent severe disease burden in overweight/obese patients and patients with genetic NPC1 impairments alike.

In chapter 2, we investigated whether interfering with dietary cholesterol absorption in the intestines via dietary plant stanol supplementation can reduce systemic symptoms of NPC1 disease. Our findings suggest that long-term consumption of plant stanols, especially at a higher dose, can reduce hepatic cholesterol accumulation, improve hepatic lipid metabolism, and reduce hepatic and systemic inflammation in NPC1 disease. Thus, this study suggests that dietary plant stanol supplementation may be an accessible tool to ameliorate metabolic dysfunction and inflammation following NPC1 impairment and lysosomal cholesterol accumulation.

In chapter 3, we aimed to determine whether dietary plant stanol supplementation can improve hepatic inflammation in the absence of excessive cholesterol intake, in a model for metabolic inflammation characterized by hematopoietic NPC1 dysfunction. While we could not fully exclude direct anti-inflammatory effects from plant stanols, this study suggests that the benefits of dietary plant stanol consumption are largely tied in with their effects on intestinal absorption of dietary cholesterol.

In chapter 4, we explored the effects of NPC1 dysfunction in bone marrow-derived immune cells on the gut microbiome. Our findings indicate that, in parallel with changes in lipid metabolism parameters, hematopoietic NPC1 dysfunction increases the diversity of the gut microbiome composition following a high fat, high cholesterol diet. This study strengthens 
the link between disturbances in lipid metabolism and gut microbiome, and further highlights the impact of host genetic mutations in the composition of the gut microbiome.

In chapter 5, we investigated whether increasing antibody levels against oxLDL by pneumococcal immunization can reduce systemic and neurological symptoms of NPC1 disease. In this study, pneumococcal immunization effectively reduced hepatic cholesterol accumulation, inflammation and apoptosis. In addition, pneumococcal immunization reduced brain inflammation and improved motor deficits in NPC1 disease mice. These results suggest that pneumococcal immunization may be an effective therapeutic tool to ameliorate NPC1 disease symptoms. In addition, our findings emphasize the role of oxLDL in NPC1 disease pathology.

In chapter 6, we conducted a clinical pilot study to analyze whether administration of a pneumococcal vaccine commonly used in the clinic (Prevenar 13) induces higher levels of anti-oxLDL IgM antibodies in patients with partial lipodystrophy, familial hypercholesterolemia, and Niemann-Pick type B. Despite having analyzed a small and heterogeneous sample of patients, our results suggest that a single dose of Prevenar 13 is not suitable to increase anti-oxLDL IgM antibody levels in humans, emphasizing the need to investigate alternative strategies to target oxLDL in patients. 


\section{Samenvatting}

In de afgelopen jaren wordt lysosomale disfunctie steeds meer erkend als een oorzakelijk factor die bijdraagt aan een breed scala van ziekten, waaronder NASH, atherosclerose, dementie en verschillende soorten kanker. Langdurige opstapeling van lysosomaal cholesterol wordt tegenwoordig beschouwd als één van de belangrijkst factoren die aan de grondslag liggen van lysosomale disfunctie, onder meer door de gestage toename van personen (volwassenen en kinderen) die lijden aan obesitas/overgewicht en het geassocieerde MetS. Metabole stoornissen die zijn geïnduceerd door een chronische ongezonde levensstijl (overmatige calorie-innanme in combinatie met verminderde fysieke activiteit) kunnen namelijk lysosomale cholesterolophoping veroorzaken, resulterend in lysosomale en cellulaire disfunctie, ontsteking en ziekte. Verder brengen schadelijke mutaties of SNP's de activiteit van het lysosomaal cholesterolefflux-eiwit NPC1 in gevaar, waardoor individuen vatbaar worden voor lysosomale cholesterolaccumulatie, metabole disfunctie en ontsteking (hetgeen zeer goed is beschreven in de context van de NPC1-ziekte. Als zodanig kan het onderzoeken van mechanismen die bijdragen aan de accumulatie van lysosomaal cholesterol inzicht bieden aan de ontwikkeling van nieuwe therapeutische strategieën om ernstige ziektelast bij patiënten met overgewicht / obesitas en patiënten met genetische NPC1stoornissen te voorkomen.

In hoofdstuk 2 hebben we onderzocht of het moduleren van de opname van cholesterol in de darmen via plantaardige stanolsuppletie in de voeding de systemische symptomen van de NPC1 ziekte kan verminderen. Onze bevindingen suggereren dat langdurige consumptie van plantenstanolen, vooral bij een hogere dosis, de accumulatie van cholesterol in de lever kan verminderen, het lipidenmetabolisme in de lever kan verbeteren en hepatische en systemische ontsteking bij de ziekte van NPC1 kan verminderen. Deze studie suggereert verder dat toevoeging van plantaardige stanolen in het dieet een toegankelijk hulpmiddel kan zijn om metabole disfunctie en ontsteking te verminderen in NPC1-stoornissen en stoornissen gelinkt aan lysosomale cholesterolophoping.

In hoofdstuk 3 bepaalden we of toevoeging van plantaardige stanolen in de voeding leverontsteking kan verbeteren bij afwezigheid van overmatige cholesterolinname, in een model voor metabole inflammatie gekenmerkt door hematopoëtische NPC1-disfunctie. Hoewel we de directe ontstekingsremmende effecten van plantenstanolen niet volledig konden uitsluiten, deze studie suggereert dat de voordelen van de consumptie van plantaardige stanolen in de voeding grotendeels samenhangen met hun effecten op de opname van cholesterol in de darmen. 
In hoofdstuk 4 hebben we de effecten van NPC1-disfunctie in uit-beenmerg-afgeleideimmuuncellen op het darmmicrobioom onderzocht. Onze bevindingen geven aan dat, parallel met veranderingen in de parameters van het lipidenmetabolisme, hematopoëtische NPC1-disfunctie de diversiteit van de samenstelling van het darmmicrobioom verhoogt na een dieet met een hoog vetgehalte en een hoog cholesterolgehalte. Deze studie versterkt het verband tussen verstoringen in het lipidenmetabolisme en het darmmicrobioom, en benadrukt verder de impact van genetische mutaties van de gastheer op de samenstelling van het darmmicrobioom.

In hoofdstuk 5 hebben we onderzocht of het verhogen van antilichaamspiegels tegen oxLDL door pneumokokkenimmunisatie de systemische en neurologische symptomen van de NPC1-ziekte kan verminderen. In deze studie verminderde pneumokokkenimmunisatie effectief de accumulatie van cholesterol, ontsteking en apoptose in de lever. Bovendien verminderde pneumokokken-immunisatie hersenontsteking en verbeterde motorische tekorten bij muizen met de ziekte van NPC1. Deze resultaten suggereren dat immunisatie met pneumokokken een effectief therapeutisch hulpmiddel kan zijn om de symptomen van de NPC1-ziekte te verlichten. Bovendien benadrukken onze bevindingen de rol van oxLDL in de pathologie van de ziekte van NPC1.

In hoofdstuk 6 hebben we een klinische pilootstudie uitgevoerd om te analyseren of toediening van een pneumokokkenvaccin dat vaak in de kliniek wordt gebruikt (Prevenar 13), hogere niveaus van anti-oxLDL IgM-antilichamen induceert bij patiënten met partiële lipodystrofie, familiaire hypercholesterolemie en Niemann-Pick-type. B. Ondanks dat we een kleine en heterogene steekproef van patiënten hebben geanalyseerd, suggereren onze resultaten dat een enkele dosis Prevenar 13 niet geschikt is om de anti-oxLDL IgMantilichaamspiegels bij mensen te verhogen, wat de noodzaak benadrukt om alternatieve strategieën te onderzoeken om oxLDL bij patiënten te verminderen. 


\section{Impact paragraph}

Scientific and socio-economic advances have brought on a number of innovations that have irremediably transformed human lives. While many of these advances have improved the average individual's quality of life and lifespan, they carry their own set of challenges, such as high levels of stress and loneliness, as well as the increasing prevalence of metabolic diseases. A staggering portion of the population suffers from, or will at some point develop, overweight/obesity and Metabolic Syndrome. In 2016, the World Health Organization estimates that 1.9 billion adults were overweight, 650 million of which were obese (1). In addition, nearly 378 million adolescents and children under five years of age are either overweight or obese worldwide $(1,2)$. Furthermore, it is estimated that nearly a quarter of the population presents Metabolic Syndrome, a group of metabolic disease risk factors that includes hyperlipidemia, hyperglycemia and high blood pressure (3).

While the combination of overnutrition and low energy expenditure underlies the development of overweight/obesity and Metabolic Syndrome, there are a number of factors that can influence this ratio, including cultural and socio-economic elements; genetic variants and mutations impacting energy metabolism; and medication, as well as disability (4). Nonetheless, socio-economic factors and education are the most important contributors to the development of overweight and Metabolic Syndrome, and these disorders are becoming more and more prevalent in developing countries and low-income populations (5). Regardless of the underlying causes, the development of effective preventive strategies and therapies is more important than ever. If untreated, overweight/obese and Metabolic Syndrome patients are at high risk of developing non-communicable diseases (NCDs) such as type 2 diabetes mellitus, non-alcoholic steatohepatitis, cardiovascular disease, musculoskeletal disorders, neurodegenerative and neuropsychiatric disorders (Alzheimer's disease, depression) and several types of cancer. Altogether, the abovementioned NCDs account for more than $70 \%$ of preventable, premature deaths worldwide, and this number is expected to increase in years to come (6). Metabolic disorders and NCDs cause a tremendous burden on affected individuals and their families on both an emotional and economical level, as patients struggle to remain employed and keep up with health costs. As such, in addition to posing a threat to public health, metabolic disorders and associated NCD burden are important components of the pervasive vicious cycle of poverty, and thus interfere with socioeconomic development where it is often needed the most. Of note, health costs and loss of economic activity associated with NCDs were estimated to result in the loss of nearly 38 trillion euros between 2010 and 2030 worldwide (i.e., nearly half of the global gross domestic product in 2019) (7), further emphasizing the impact of metabolic disorders and NCDs on socio-economic development. Another important factor associated with increased 
development of metabolic disorders and associated NCDs is aging. In 2018, the estimated number of individuals over the age of 64 surpassed the number of infants for the first time in human history, and it is well known that the risk of metabolic dysfunction and development of NCDs is positively associated with aging. Ironically, although life expectancy has increased by several years in many countries worldwide, such trends are slowing down, possibly due to increased mortality associated with NCDs, among other factors (8). Nonetheless, it is undeniable that current demographics and increasing numbers of older individuals represent an important factor to the increasing prevalence of metabolic disorders and NCDs, particularly in developed countries. Of note, metabolic disorders and NCDs make patients more vulnerable to infectious diseases and associated comorbidities and mortality $(9,10)$, a fact worth mentioning in light of recent epidemic and pandemic events, such as those mediated by SARS and, recently, COVID-19. All considered, research into pathways underlying disease development, in parallel with policy changes, is essential for patients, clinicians and societies alike, in order to develop efficient preventive and therapeutical strategies to improve life quality of metabolic disease patients.

Despite the different pathophysiology of NCDs such as NASH, Alzheimer's disease and cancer, they often feature overlapping underlying pathological mechanisms, including inflammation, oxidative stress, dysfunctional energy metabolism and lysosomal dysfunction. Of note, lysosomal dysfunction is both an important trigger and contributor to disease burden, as is clear in the case of NPC1 disease, a severe genetic disease. Although rare, NPC1 disease takes a great toll in patients, triggering the development of liver, spleen and lung dysfunction. In addition, NPC1 disease patients suffer from debilitating and progressive neurodegeneration which impairs their motor and cognitive abilities. While some NPC1 disease patients suffer from relatively mild and late-onset symptoms, many experience disabling peripheral and neurological disease manifestations from a very young age. Due to a combination of disease severity, delayed diagnosis and limited therapeutical tools, NPC1 disease patients suffer from strongly reduced life quality and lifespan. As such, further research into NPC1 disease is required, in order to develop more efficient diagnostic and therapeutic strategies. In addition, given the aforementioned overlap between pathophysiological mechanisms of NPC1 disease and metabolic NCDs, findings pertaining to NPC1 disease may be applicable to a wide range of NCDs, and vice-versa. In addition, several studies have found genetic NPC1 SNPs and heterozygous NPC1 loss-of-function mutations in overweight and obese individuals, as well as patients with type 2 diabetes and cardiovascular disease. These studies further link (mild) NPC1 impairment and lysosomal lipid accumulation in highly prevalent metabolic disorders and NCDs, further emphasizing the wide application value of NPC1 disease findings. 
In this thesis, we reported for the first time that NPC1 impairment in blood-derived immune cells shifts the gut microbiome composition in a HFC diet setting (chapter 4). Future studies should further explore whether NPC1 dysfunction, as well as lifestyle-associated lysosomal lipid accumulation, is also associated with gut microbiome composition in humans. In addition, further studies should assess whether the interaction between NPC1 impairment and HFC diet results in a well-defined gut microbiome landscape. If so, the detection of such a characteristic gut microbiome via stool samples could translate into a non-invasive diagnosis tool for lysosomal cholesterol accumulation in individuals and allow for clinicians to put in practice more targeted and efficient therapeutic strategies. In addition, while the role of the gut microbiome in health and disease has yet to be fully elucidated, altered gut microbiome composition has been associated with NCDs such as liver disease and cancer. As such, it is possible that host NPC1 impairment/HFC diet and the resulting gut microbiome structure contributes to disease burden severity. Therefore, tools to reshape the gut microbiome may be promising, affordable and accessible tools to improve NPC1-related host features, such as dyslipidemia and inflammation, and thus prevent the development of severe NCDs in individuals with lysosomal cholesterol accumulation, as observed in obesity and MetS.

Furthermore, in chapter 2, we showed that increasing plant stanol consumption improves liver lipid metabolism and as well as liver and peripheral inflammation in a mouse model for NPC1 disease. Dietary plant stanol supplementation, via supplements or plant stanolenriched margarines, is a tool commonly used to reduce hypercholesterolemia in patients. As such, it is a widely available and affordable tool which may be beneficial to a portion of NPC1 disease patients, in combination with other therapeutic compounds. Of note, dysphagia, i.e., difficulties swallowing, is a symptom that many NPC1 disease patients suffer from; as such, dietary supplementations and oral pharmacological interventions are unlikely to be applicable to patients with severe NPC1 disease. Nonetheless, our findings from chapter 2 indicate that dietary plant stanol supplementation is an effective tool to reduce liver inflammation in a disease model characterized by severe lysosomal lipid accumulation. This observation suggests that dietary plant stanol supplementation may be a valuable tool to prevent or reduce systemic inflammation in a wider range of NCDs characterized by lysosomal lipid accumulation, such as obesity, MetS and NASH, in addition to reducing hypercholesterolemia.

Moreover, in chapter 5, we demonstrated that increasing antibody levels against oxLDL reduces systemic and neurological symptoms of NPC1 disease. While oxLDL is not a primary source of NPC1 disease, our findings emphasize that oxLDL contributes to NPC1 disease severity by exacerbating metabolic dysfunction, oxidative stress and inflammation. As such, 
reducing oxLDL levels may remove further challenges (i.e. inflammation, oxidative stress) that altogether contribute to further lysosomal dysfunction and severe tissue and organ dysfunction. Indeed, previous studies found that compounds targeting parallel disease mechanisms in many cases work in synergy, enhancing the overall benefits regarding disease progression and burden. As such, the combination of tools targeting parallel disease mechanisms is likely to benefit individuals with complex diseases for which there are no definite curative treatments, such as obesity and MetS and related NCDs.

Similarly to NPC1 disease, high levels of oxLDL have been associated with the progression of highly prevalent NCDs, such as NASH, cardiovascular disease, Alzheimer's disease and some types of cancer. As such, tools to reduce oxLDL show great promise to improve quality of life for a great number of patients. Importantly, while increasing antibody levels against oxLDL may be an effective tool to reduce inflammation, oxidative stress and overall disease burden, our observations from chapter 6, in line with reports from other investigators, indicate that pneumococcal vaccination does not increase antibodies against oxLDL in human patients. As such, our results emphasize the need to develop alternative strategies to target oxLDL in human patients, such as vaccines tailored specifically to trigger antibodies against oxLDL or even nano compounds targeting this molecule.

Overall, the findings reported in this thesis highlight the consequences of lysosomal lipid accumulation and the potential of different therapeutic strategies in ameliorating NPC1 disease. In addition to being applicable to NPC1 disease, such findings may also come to benefit patients with metabolic disorders and associated NCDs. 


\section{REFERENCES}

1. WHO. Obesity and overweight 2020. Available from: https://www.who.int/newsroom/fact-sheets/detail/obesity-and-overweight. Accessed on: 21/05/2021

2. Di Cesare M, Sorić M, Bovet P, Miranda JJ, Bhutta Z, Stevens GA, et al. The epidemiological burden of obesity in childhood: a worldwide epidemic requiring urgent action. BMC Med. 2019;17(1):212.

3. Saklayen MG. The Global Epidemic of the Metabolic Syndrome. Current Hypertension Reports. 2018;20(2):12.

4. Blüher M. Obesity: global epidemiology and pathogenesis. Nature Reviews Endocrinology. 2019;15(5):288-98.

5. Wang Y, Wang J. Modelling and prediction of global non-communicable diseases. BMC Public Health. 2020;20(1):822.

6. WHO. Noncommunicable diseases: Key Facts 2018.Last updated on: 13/04/2021. Available from: https:/www.who.int/news-room/fact-sheets/detail/noncommunicablediseases. Accessed on: 05/05/2021

7. David E. Bloom EC, Eva Jané-Llopis, Shafika Abrahams-Gessel, Lakshmi Reddy Bloom, Sana Fathima, Andrea B. Feigl, Tom Gaziano, Ali Hamandi, Mona Mowafi, Danny O'Farrell. The Global Economic Burden of Noncommunicable Diseases. PGDA Working Papers; 2012.

8. Crimmins EM. Recent trends and increasing differences in life expectancy present opportunities for multidisciplinary research on aging. Nature Aging. 2021;1(1):12-3.

9. Smith M, Honce R, Schultz-Cherry S. Metabolic Syndrome and Viral Pathogenesis: Lessons from Influenza and Coronaviruses. Journal of Virology. 2020;94(18):e00665-20.

10. Cefalu WT, Rodgers GP. COVID-19 and metabolic diseases: a heightened awareness of health inequities and a renewed focus for research priorities. Cell Metabolism. 2021;33(3):473-8. 


\section{Acknowledgments}

They say it takes a village to raise a child, and the same can certainly be said about a PhD student. While this book consists of the research output of my $\mathrm{PhD}$ journey, it also represents the work, companionship, and support from many kind individuals. Hereby, I would like to thank everyone who made my PhD journey a less bumpy and more enjoyable ride, one way or another.

First, I would like to express my gratitude towards my supervisor, Ronit. We first met virtually about five years ago, when I was at the beginning of my internship in Japan. You and Jan were then teaching us how to design and communicate scientific research, and your warm energy back then made these sessions much more enjoyable, even though they were quite late for us due to the time difference. After that, you remained a lifeline back in Maastricht, and I contacted you again some months later with concerns regarding the limited output of my internship. Even though your response soothed my anxiety, you also managed to shock me when you asked in P.S. if I wanted to interview for a $\mathrm{PhD}$ position in your lab. I had just told you that I wasn't performing as well as I wanted to, and there you were, considering me to become part of your team. I think this little origin story tells a lot about you, Ronit; about your kindness, and how you see the best in people, even when they don't see it themselves. Through the years, I have also come to know, admire, and respect your dedication to work and to the people around you. You went through a lot of personal challenges (quite the euphemism, but there you go), which you fortunately overcame. Despite it all, I never once thought you absent; you were always there, working tirelessly in the background, and making sure we could count on you for input and guidance. Thank you for believing in me, for all the lessons in being a better, more critical scientist, and for all your support through the years. You are a strong, positive person, who always finds a way to solve, well, anything, and I couldn't have asked for a better mentor. I wish you, your husband and your beautiful children all the happiness and success going forward.

Of course, I also want to thank Tom, my daily supervisor these past four years. Where to even begin, Thomas. The term "daily supervisor" is not in the least an exaggeration; I'm afraid it may even be an understatement. Regardless of the context, regardless of the time of day, I could always count on you for advice, for guidance, for a break filled with philosophical discussions and, of course, many, many absurd jokes. I will always remember how, at the beginning of my PhD, you played a prank on me in the animal room (something to do with, huh huh, bowel movements); it was hilarious, and still makes me smile. You are truly one of a kind, and I could go on, and on, ad nauseam really, about how committed you are to your work; about how you're not afraid to think outside of the box; about how patiently you listen; 
and about how generous you are with your time, whether it's about giving advice regarding data, manuscripts, experiments, lab analyses, tutoring, posters, presentations, etc, etc, etc. Through it all, even when you were in Strasbourg, even when you were having difficult issues, I could count on your support and your honest feedback, and I honestly can't thank you enough for it. I'm very happy to have met you and will always take you as an example to follow on many fronts. Thank you for putting up with me, and helping me become a better scientist. Please take good care of yourself, and I wish you all the happiness and success this world can offer. Not that you need anyone's wishes; I'm not sure what the future brings, but I know you'll make the most of it (the future should be very, very afraid).

Tulasi and Lingling, my dear PhD buddies!! What a ride it has been. The three of us started our PhD within some months of each other, and I'm officially the last one to graduate (we did it!). I'm so proud of both of you, and as I'm writing this, I can only hope to do as well as you.

Lingling, I remember when you first joined us in Maastricht. There were a lot of challenges, of course, but you quickly improved your English and adapted to Europe; and if these were impressive feats at the time, it is now no wonder. You are an incredibly positive and caring soul, always willing to give a sympathetic ear and a patient, encouraging word to those around you. You were always exceptionally hard working and driven, to the point where you finished your PhD many months in advance; I can't tell you how much I admire that. Thank you for always helping me in the lab, for our conversations and laughs and for always seeing the positive side of things (not to mention the delicious hot pot and dumplings ()). I wish you, Lichuan and your beautiful daughter a happy life, and all the success back in China. Whether here or there, I look forward to meeting you again!

Tulasi, my wonderful paranymph and dear girlfriend (oops, secret is out). It wasn't easy for you as well to pack up and start a new life in Maastricht, but I'm so glad you did (thanks, Deepak!). You're a true scientist, always keeping up with literature and thinking of new ways to answer questions; in fact, you're one of the most knowledgeable people I know, and I could always come to you for advice or brainstorming. Not only that, you're also incredibly hardworking, and despite all the stress and the "busy" times (ah, understatement of the century), you were always one of the most reliable and funny people to be around of; thank you for all the laughs and shared jokes throughout the years, they made the dark, rainy days at the office much brighter. Whether regarding experiments or food, thank you for being such a generous and kindhearted person. I know you're going to do great in your post-doc, and I look forward to hearing about your future adventures with Deepak, in the mountains and beyond. I wish you and your family all the happiness and success. 
I would also like to acknowledge and thank all the other wonderful members of the Metabolism Masters group. Albert, you're incredibly knowledgeable and always ready for debating ideas, and I'm going to miss our conversations on everything, from videogames to politics and the future of humanity. Thank you for all the advice on research and always being so patient and kind. Actions speak louder than words, and regardless of how much you "don't care", at the end of the day, we could always count on your support. Thank you. I wish you all the best. Please be careful with your board, and here's to you finding a cold place to settle down and finally finish your campaigns without having to worry about sudden deadlines. Yvonne, you're such a warm, kind and hardworking person, and I'm very glad to have worked alongside you for my first couple of years. Regardless of how busy you were, I could always count on you for advice or help in the lab and with analyses. Thank you for all your support, especially with the Prevenar project; God knows it wasn't easy to navigate, but your organization and eye for detail made the process much smoother. I wish you a lot of success and a very happy life with Joel and Gisèle. Dennis, from your awesome taste in music (always playing in the office or in the lab, of course); to your colorful socks, as well as the stories about your pussy cat; you always bring joy to the office, and working alongside you has been a pleasure. Thank you for your help with double checking data, for putting up with me forgetting where the glass disposal is for the billionth time, and for your advice and help, from the trips to Genk, to PBMC isolation and organizing blood draws. Rock on $\backslash \mathrm{m} /$, and I wish you, Anne and Julia many, many wonderful years ahead. Annemarie and Mengying, although you both joined our group almost a year ago, I unfortunately did not have the chance to get to know you a lot, due to the COVID situation. However, I enjoyed hanging out with you during our online and few in-person outings. I wish you all the best, and I'm sure you're going to make the most out of your PhD journeys. Finally, I would also like to thank our interns throughout the years, including Erik and Leoni. Erik, thank you for all the laughs, you truly brought new life and energy to the office. I wish you all the best with your degree and your life ahead. Leoni, you were the first (and only) student I (co-)supervised. I'm sure there's a lot I can improve on, but I can't stress enough how easy you made my job. For months, you were always one of the first people in the office, and one of the last to leave, making sure that all the lab work was done well and on time, and you always brough a positive atmosphere to the office. Thank you for your help with the plant stanols' analyses. I'm sure you'll do great things in the future, and I wish you a lot of happiness and success back in Leuven and beyond.

I would also like to express my gratitude to all the members of the Molecular Genetics department for their impact. Petra, thank you for organizing so many activities throughout the years for us, and for your support and help with all sorts of matters. Jan, you were always very kind and positive, and it was a pleasure to chat with you in social gatherings and lab 
chats. Joost, you have such a big personality and you bring so much energy around you, it's contagious. Thank you for always being so cheerful, and for all your input throughout our lab chats. Miranda, thank you for your kindness and positive energy, it was always a pleasure to talk to you. Willem, thank you for your input during the lab chats; you're sometimes tough, but always fair, and I appreciated your advice throughout the years. Agnieszka, you always brought a smile to my face (even in the morning, before I'd had my coffee, which is very difficult). Thank you for your warm, positive energy, for your kindness and for your help with protocols and orders. Many thanks to Ilvy, Shujin, Aomin, Francesco, Jobran and LiYen for their kindness and for making such a positive atmosphere around the office and the lab. You're all talented, hardworking people, and I wish you all the success and happiness going forward.

Big thanks as well to all the co-authors and collaborators of the studies included in this thesis, including, but of course not limited to: Hellen Steinbusch, for her generous advice and all the sessions spent at the microscope debating about brain slices, as well as her advice on neuro-related lab protocols; Prof. Dr. Jogchum Plat and Prof. Dr. Dieter Lütjohann, for their knowledgeable advice and critical input regarding plant stanols and sterol metabolism; Prof. Dr. David Cassiman, for his input and collaboration in organizing and conducting the Prevenar study; Annick Vanclooster and Nele Vanhoutvin, also for their valuable contributions to the Prevenar study.

I would also like to extend huge thanks to Prof. Dr. Frederik-Jan van Schooten, Dr. Sabine Baumgartner, Prof. Dr. David Leake, Dr. Kaatje Lenaerts, Dr. Frank Pfrieger and to Prof. Dr. Marc van Zandvoort, for being part of my assessment committee and thus generously sharing their time and critical input. I look forward to meeting you and discussing the contents of this thesis in January.

Thank you to my friends, close and far away, for their companionship and for all the laughter, support and shared experiences throughout the years. I am a very lucky person to have met you and to have spent so many great (and some not so great) times by your side. Here's to a swift end to the lockdown life and to more time spent together for many years to come (-) Although I can't mention everyone, a special thanks to Tate, for being my paranymph, not to mention for all the coffee breaks, movie nights, day trips, etc, as well as for introducing me to the Social Committee. You were a constant those first couple of years and made my life much richer in Maas; thank you. Here's to keep seeing each other and hanging out more (and here's to finding the next Love Witch and Baahubali; we can do it!). 
Quase por último, mas mais importante, quero agradecer à minha família por todo o amor e apoio. Mudar de país deu-me muitas experiências de valor, mas custou-me estar perto de vós, e não é um custo baixo. Tenho saudades vossas todos os dias, e não tenho palavras para expressar o quão grata estou por ter tido a sorte de nascer entre vós; amo-vos muito. Um obrigada especial à minha mãe e ao meu pai, por me terem dado uma vida privilegiada em tantos aspectos, e por me terem sempre encorajado a ser mais e melhor. Esta pode não ser uma tese merecedora de um Nobel, mas representa o fim de uma jornada cheia de esforço, dedicação e obstáculos ultrapassados; e grande parte desta jornada ter sido começada e acabada é toda vossa. Obrigada pelo amor incondicional, por todos os ensinamentos e todas as oportunidades; espero continuar a crescer e a fazer-vos orgulhosos.

Finally, my dear Bogdan (no nicknames, this is a serious document!). I met you only three years ago, but somehow, it's all it took for you to become one of the most important people in my life. You make everything, from the mundane to the thrilling, so, so much better. Thank you for being by my side, for looking out for me, for making me laugh until I nearly burst out, for all the music, all the conversations, all the companionship and all the adventures. I don't know much about the future, but I'm grateful for the chance to share it with you. Te iubesc cel mai mult. 


\section{Curriculum vitae}

Inês Reis was born on February 28 $8^{\text {th }}, 1993$ in Lisbon, Portugal. Between 2011 and 2015, she enrolled in the Molecular and Cellular Biology Bachelor's degree at NOVA University of Lisbon. Having spent four years diving into molecular networks and basic research, Inês decided to expand her knowledge on human disease by pursuing a Master's degree in Biomedical Sciences in Maastricht University (Maastricht, The Netherlands). During her Junior Professional Training course, she spent six months investigating the role of GABA receptors in temporal lobe epilepsy, under the supervision of Dr. Govert Hoogland and Dr. Sandra Schipper (MhENS, School for Mental Health and Neuroscience of Maastricht University). During the second year of her Biomedical Sciences MSc, Inês moved from to Sendai, Japan, as part of the Double European-Japan Master's Degree Program in Neurosciences. During this time, in addition to learning about the Japanese culture, Inês performed a ten month-long internship at the Department of Developmental Neurosciences of the Graduate School of Medicine from Tohoku University. Under the supervision of Prof. Dr. Noriko Osumi and Dr. Hitoshi Inada, Inês investigated the role of fatty acid-binding protein 7 and polyunsaturated fatty acids in astrocyte proliferation during brain development.

After completing her MSc degree in 2017, Inês returned to Maastricht University to join Prof. Dr. Ronit Shiri-Sverdlov's team at the Molecular Genetics and Cell Biology Department as a $\mathrm{PhD}$ candidate. Asides from her work as a PhD candidate, she took on tutor roles in Problem Based Learning sessions for several Bachelor's degree courses. In addition, she supervised a Bachelor student in collaboration with her $\mathrm{PhD}$ co-supervisor, Dr. Tom Houben. Furthermore, she joined the Social Committee and acted as treasurer for the PhD Academy of Maastricht University, helping to organize a number of networking events and workshops aimed at PhD students. 

